Universitat Politècnica de València

Departamento de Comunicaciones

Valencia Nanophotonics Technology Center

Ph.D. Thesis
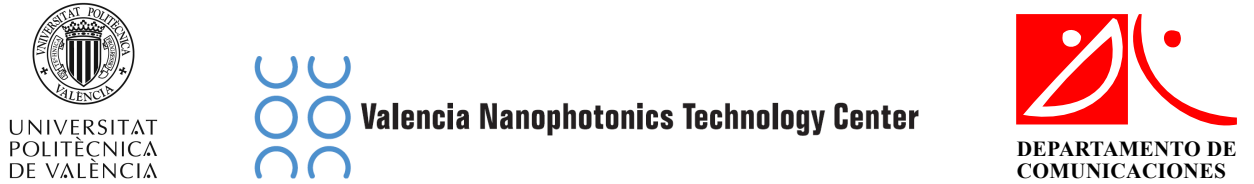

\title{
Passive and active silicon photonics devices at TLC telecommunication wavelengths for on-chip optical interconnects
}

Author:

Andrea Zanzi
Supervisors:

Dr. Antoine Brimont

Dr. Javier Martí Sendra 

Dedicated to Sara and my parents Thank you for believing in me... 



\section{Resumen}

Las tecnologías ópticas son el eje vertebrador de los sistemas de comunicación modernos que proporcionan acceso de alta velocidad a la Internet, interconexiones eficientes entre centros de datos y dentro de ellos. Además, se están expandiendo hacia campos de investigación crecientes y nuevos mercados como son las aplicaciones de comunicaciones por satélite, los LIDAR (Laser Imaging Detection and Ranging), la computación neuromórfica y los circuitos fotónicos programables, por nombrar algunos. La fotónica de silicio está considerada y aceptada ampliamente como una de las tecnologías clave para que dichas aplicaciones puedan desarrollarse. Como resultado, hay una fuerte necesidad de estructuras fotónicas básicas integradas que sean innovadoras, que soporten altas velocidades de transmisión y que sean más eficientes en términos de consumo de potencia, a fin de aumentar la capacidad de los circuitos integrados fotónicos de silicio.

El trabajo desarrollado y presentado en esta tesis se centra en el diseño y la caracterización de dispositivos avanzados pasivos y activos, para circuitos fotónicos integrados. La tesis consta de tres capítulos principales, así como de sendas secciones de motivación y conclusiones que exponen los fundamentos y los logros de este trabajo. El capítulo uno describe el diseño y la caracterización de un modulador electro-óptico Mach-Zehnder incorporado en una unión pn vertical altamente eficienciente que explota el efecto de dispersión de plasma en banda O. El capítulo dos está dedicado al diseño y caracterización de una nueva geometría de dispositivo de interferencia multimodo asimétrico y su aplicación en un modulador Mach-Zehnder. El capítulo tres está dedicado al diseño y caracterización de innovadores cristales fotónicos unidimensionales para aplicaciones de modulación con luz lenta. Se presenta un amplio análisis de los principales retos derivados del uso de la misma. 


\section{Resum}

Les tecnologies òptiques són l'eix vertebrador d'aquells sistemes de comunicació moderns que proporcionen accés d'alta velocitat a la Internet, així com interconnexions eficients inter i entre centres de dades. A més a més, s'estan expandint cap a camps d'investigació creixents i nous mercats com són les aplicacions de comunicacions per satèl-lit, els LIDAR (Laser Imaging Detection and Ranging), la computació neuromòrfica i els circuits fotònics programables, entre d'altres. La fotònica de silici és considerada i acceptada àmpliament com una de les tecnologies clau i necessàries perquè aquestes aplicacions puguen desenvolupar-se. Per aquest motiu, es fa necessària l'existència d'estructures fotòniques bàsiques integrades que siguen innovadores, que suporten altes velocitats de transmissió i que siguen més eficients en termes de consum de potència, a fi d'augmentar la capacitat dels circuits integrats fotònics de silici. El treball desenvolupat i presentat en aquesta tesi se centra en el disseny i la caracterització de dispositius avançats passius i actius, per a circuits fotònics integrats. La tesi consta de tres capítols principals, així com d'una secció de motivació i una altra de conclusions que exposen els fonaments i els assoliments d'aquest treball. El capítol u descriu el disseny i la caracterització d'un modulador electro-òptic Mach-Zehnder incorporat en una unió pn vertical d'alta eficiència que explota l'efecte de dispersió de plasma en la banda O. El capítol dos està dedicat al disseny i caracterització d'una nova geometria de dispositiu d'interferència multimode asimètric així com a la seua aplicació en un modulador Mach-Zehnder. El capítol tres està dedicat al disseny i caracterització d'innovadors cristalls fotònics unidimensionals per a aplicacions de modulació amb llum lenta. S'inclou també una anàlisi detallada dels principals reptes derivats de l'ús d'aquest tipus de llum. 


\section{Abstract}

Optical technologies are the backbone of modern communication systems providing high-speed access to the Internet, efficient inter and intra-data center interconnects and are expending towards growing research fields and new markets such as satellite communications, LIDARs (Laser Imaging Detection and Ranging) applications, Neuromorphic computing, and programable photonic circuits, to name a few. Because of its maturity and low-cost, silicon photonics is being leveraged to allow these new technologies to reach their full potential. As a result, there is a strong need for innovative, high-speed and energy-efficient photonic integrated building blocks on the silicon platform to increase the readiness of silicon photonic integrated circuits. The work developed and presented in this thesis is focused on the design and characterization of advanced passive and active devices, for photonic integrated circuits. The thesis consists of three main chapters as well as a motivation and concluding sections exposing the rationale and the accomplishments of this work. Chapter one describes the design and characterization of an electro-optical Mach-Zehnder modulator embedded in highly efficient vertical pn junction exploiting the free-carrier dispersion effect in the O-band.. Chapter two is devoted to the design and characterization of a novel geometry of asymmetrical multimode interference device and its implementation in a Mach-Zehnder modulator. Chapter three is dedicated to the design and characterization of innovative 1-dimensional photonic crystal designs for slow- lightmodulation applications. An extensive analysis of the main trade-off arising from the use of slow light is presented. 


\section{Acknowledgements}

First of all, I would like to express my deepest thank and gratitude to my supervisors Prof. Javier Marti and Dr. Antoine Brimont for allowing to do my PhD under their supervision. I am very grateful for the opportunity to work at the NTC for all these years. Thanks to you for allowing to grow both as a person and a scientist, without your guidance this work would not been possible.

Secondly, I want to thank you the Generelitat Valenciana and the European Project L3MATRIX for the funding, without them my doctorate would not taken place.

My deepest gratitude goes to the L3MATRIX consortium for the possibility to be part of the team for 3 years, and also for the possibility to see my designs come to reality. In the same way, I want to thank you the members of the NTC's clean room. Thank you for allowing me to push my ideas to the extreme, and try always your best to fabricate what I was asking.

Furthermore, I would like to thank all the current and former members of the NTC for the unforgettable years, but a special thanks goes to a restrict number of people that now I can call friends. Thank you guys!

Last but not least, I want to thank my parents, my girlfriend Sara, her parents and my friends. Thank you for your endless support, for your suggestions, for your guidance and for the endless encouragement to continue also in the deepest hours. Thank you for believing in me! 


\section{Contents}

Resumen

Resum iii

\begin{tabular}{lll}
\hline Abstract & V
\end{tabular}

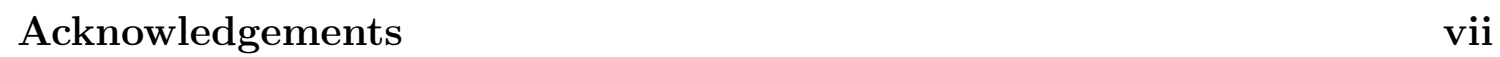

Table of contents $\quad x$

$\begin{array}{ll}\text { Motivation } & 5\end{array}$

\begin{tabular}{lll}
\hline 1 & Silicon Modulators & 7
\end{tabular}

1.1 Introduction . . . . . . . . . . . . . . . . . . . . . 7

1.2 Design Passive Components . . . . . . . . . . . . . . . . . . . . . 9

1.3 Phase shifter and modulator design . . . . . . . . . . . . . . . 14

1.4 Fabrication and Measurements . . . . . . . . . . . . . . . . . . 23

1.5 Conclusions . . . . . . . . . . . . . . . . . . . . . . . . . 30

2 Asymmetrical MMI 35

2.1 Background and design . . . . . . . . . . . . . . . . . . . 35

2.2 Measurements and Analysis $\ldots \ldots \ldots$. . . . . . . . . . . . 37

2.3 Modulator with A-MMI . . . . . . . . . . . . . . . . . . . . . . . . . . . 41

2.4 Modulator with A-MMI results . . . . . . . . . . . . . . . . . . 43

2.5 Conclusions . . . . . . . . . . . . . . . . . . . . . . 47

3 1D Photonic crystal design 53

3.1 Background . . . . . . . . . . . . . . . . . . . . . 53 
3.2 Slow light structure design . . . . . . . . . . . . . . . . . . . . 55

3.3 Single Apodization $\ldots \ldots \ldots$. . . . . . . . . . . . . . . . . . . . . 59

3.3 .1 Setup . . . . . . . . . . . . . . . . . . 61

3.3 .2 Results . . . . . . . . . . . . . . . . . . . . . 61

3.3 .3 Results - Partial Tapers . . . . . . . . . . . . . . 70

3.4 Double Taper Apodization . . . . . . . . . . . . . . . . . . . . . . 74

3.4 .1 Double Taper Design . . . . . . . . . . . . . . . . . 75

3.4 .2 Double Taper - Measurements and Discussion . . . . . . . . . 78

3.5 Conclusions . . . . . . . . . . . . . . . . . . . . . . . . . . . . . . 85

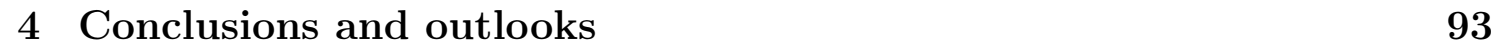

\begin{tabular}{ll}
\hline Appendices & 95
\end{tabular}

\begin{tabular}{|ll}
\hline A 1D-PhC Graph Appendix & 97
\end{tabular}

\begin{tabular}{ll}
\hline B Silvaco code & 101
\end{tabular}

B.1 ATHENA \& ATLAS simulation code . . . . . . . . . . . . . . . . . . 101

\begin{tabular}{lrr}
\hline C & FDTD & 109
\end{tabular}

C.1 2D-FDTD Code . . . . . . . . . . . . . . . . . . . . 109

\begin{tabular}{lr}
\hline D Mode Solver 2D & 121
\end{tabular}

D.1 Code Mode Solver. . . . . . . . . . . . . . . . . . . . . . . . . . . . . 121

\begin{tabular}{lll}
\hline E & Publications & 135
\end{tabular}

\begin{tabular}{lr}
\hline List of Figures & 142
\end{tabular}

\begin{tabular}{lr}
\hline List of Tables & 144
\end{tabular} 


\section{Motivations}

Since the discovery of the transistor in 1947, Electronics has become a pervasive technology in our modern lives. Photonics, on the one hand, is the technology that has allowed the modern fiber optics-based internet, the invention of the LASERs and many types of lightning devices commonly used in our everyday lives, as well as biomedical, imaging systems to improve global healthcare. Both technology are facing significant challenges, however, many roadmaps and already exisiting technologies show that taking the best of both world is the way to go. It is already seen in the Telecom and Datacom world where the ever-exponential need for large bandwidth in telecom and data centres, handheld devices and IoT (Internet of Things), and the resulting increase in power consumption require new technologies and integration to face this new challenge (Fig. 1] [1].

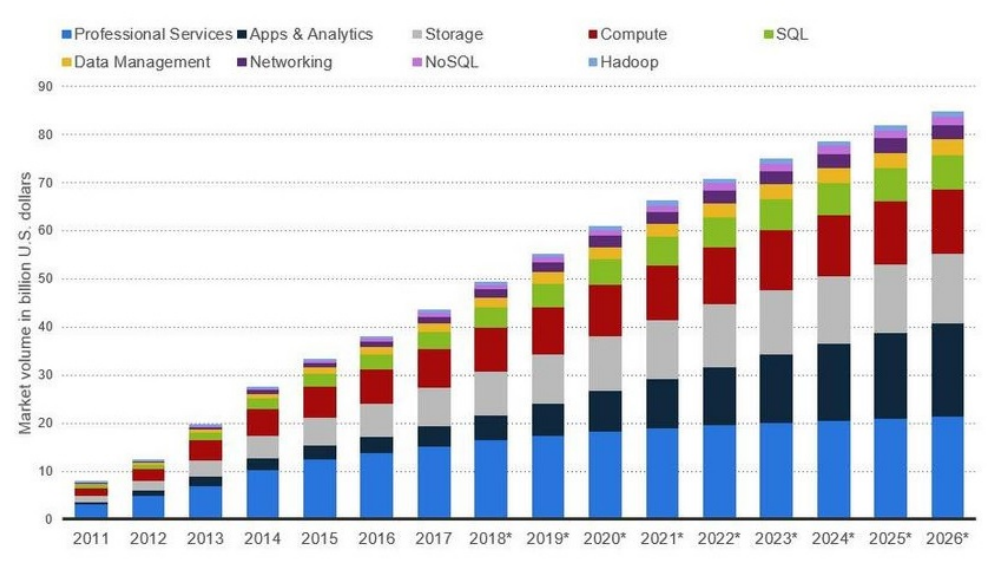

(a)

Figure 1: Big data traffic market forecast [1].

Silicon photonics is widely accepted as a key technology for the next generation 
of telecommunication systems and data interconnects [2], due to incredible advantages that integration of photonics and CMOS (complementary metal-oxidesemiconductor) will bring in terms of integration, high data rate and low-cost manufacturing costs, which comes using the conventional silicon integrated circuit infrastructure.

The high potential of photonic integrated circuits (PIC) was firstly recognized by the first studies in the mid-80s during the investigation of waveguides on siliconon-insulator (SOI) wafer structures [3]. The first applications came during the 90s for the sensor market and wavelength-division-multiplexing (WDM) telecommunications products [4]. These first applications, even if not ground-breaking, proved the feasibility of PICs and their commercial potentials and opened the road for future technologies based on silicon photonics and CMOS integration.

The $\mathrm{SiPh}$ market is forecasted to grow from 4 billion dollars in 2018 to around 19 billion dollars in 2024, with volumes increasing from around 30 million units to around 160 million units, in the same time frame [5] (Fig. 2). The growth of this market can be attributed to rising demand for silicon photonics in data centres and the growing requirement of high bandwidth and high data transfer capabilities.

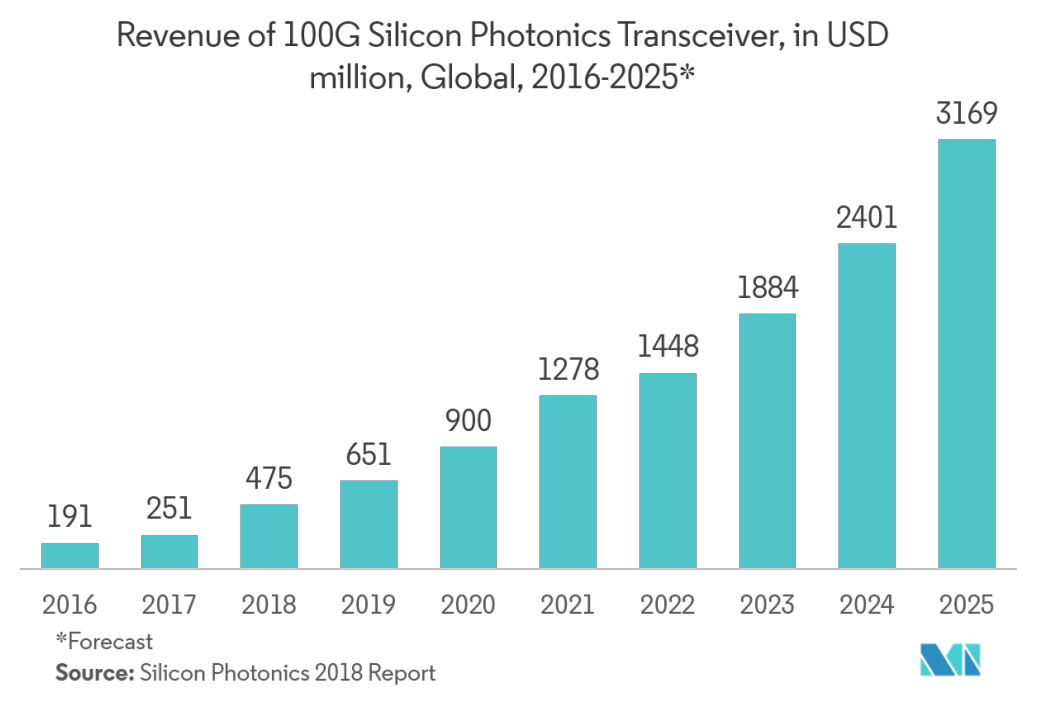

(a)

Figure 2: Forecast for silicon photonics market [5] (Silicon Photonics 2018 Report).

Therefore this field requires further development to achieve the target and be able 
to cope with the continuous growing demand of the market. As a result of this, the requests and needs for new and more efficient designs are always present. This work aims to investigate, propose and realise novel passive and active components for photonic integrated circuits on SOI technology, to further increase the readiness of the technology. 
CONTENTS 


\section{Bibliography}

[1] https://www.forbes.com/sites/louiscolumbus/2018/05/23/10-charts-that-willchange-your-perspective-of-big-datas-growth/

[2] Li, Y., Zhang, Y., Zhang, L. and Poon, A.W., 2015. Silicon and hybrid silicon photonic devices for intra-datacenter applications: state of the art and perspectives. Photonics Research, 3(5), pp.B10-B27.

[3] Hattori, K., Kitagawa, T., Oguma, M., Ohmori, Y. and Horiguchi, M., 1994. Erbium-doped silica-based waveguide amplifier integrated with a 980/1530 nm WDM coupler. Electronics Letters, 30(11), pp.856-857.

[4] Soref, R.A. and Lorenzo, J.P., 1985. Single-crystal silicon: a new material for 1.3 and $1.6 \mu \mathrm{m}$ integrated-optical components. Electronics Letters, 21(21), pp.953954.

[5] https://www.mordorintelligence.com/industry-reports/photonics-marketmarket 


\section{Chapter 1}

\section{Silicon Modulators}

In this chapter we present the design and characterization of a Mach-Zehnder modulator (MZM) featuring a vertical pn junction, which exploits the free-carrier dispersion effect for the modulate a continuous-wave optical signal.

\subsection{Introduction}

Silicon photonics modulators have been in development for more than a decade since the first reported finding regarding the $\mathrm{GHz}$ modulation frequency in a SOI waveguides [1, 2]. Driven by increased requirements for IoT (Internet-of-Things) [3], big data applications [4] and high-performance computing [5], SOI modulators have been investigated and used to accomplish the high modulation efficiency, larger bandwidth, low loss and low power consumption and advanced modulation format these application requires. Moreover, as the circuitry of a transceiver consumes the majority of the available optical power link, silicon photonics modulators, with their high modulation efficiency, play an important role for saving power consumption and therefore allow the realization of energy-efficient PICs.

Most of current SOI modulators can achieve up to tens of $\mathrm{GHz}$ of electro-optic bandwidth through the optimization of modulation mechanics, doping profiles and driving electrodes, together with advanced and precise process techniques. Monolithic integration of SOI modulators with coupled drivers built in CMOS processes have been reported in [6, 7, 8], allowing commercially available transceiver modules featuring data transmission of up to 100 and $400 \mathrm{~Gb} / \mathrm{s}$, which is in line with the 
data centre application roadmap [9, 10, 11, 12].

Mainly, the properties of silicon can be changed by properly changing the real and imaginary parts of the refractive index. The two most common methods for engineering the refractive index in SOI modulators are: the thermo-optic effect and the plasma dispersion effect. The thermo-optic effect is usually used in low-speed optical switches [13, 14] or phase control driven by slow electrical signals [15]. The high-speed silicon photonics modulators are operated based on the plasma dispersion effect. The changes of refractive index and absorption, due to the variation in free carrier concentration, are better described by Soref modification to Drude's model [16]:

$$
\begin{gathered}
\Delta n=\frac{-q^{2} \lambda_{0}^{2}}{8 \pi^{2} c^{2} n \epsilon_{0}}\left(\frac{\Delta N_{e}}{m_{c e}^{*}}+\frac{\Delta N_{h}}{m_{c h}^{*}}\right) \\
\Delta \alpha=\frac{-q^{3} \lambda_{0}^{2}}{4 \pi^{2} c^{3} n \epsilon_{0}}\left(\frac{\Delta N_{e}}{m_{c e}^{* 2} \mu_{e}}+\frac{\Delta N_{h}}{m_{c h}^{* 2} \mu_{h}}\right)
\end{gathered}
$$

where $q, c, \epsilon_{0}, n$, and $\lambda_{0}$ are the elementary electronic charge, speed of light in vacuum, permittivity of free space, unperturbed material refractive index, and light wavelength in vacuum, respectively. $\Delta N_{e}$ and $\Delta N_{h}$ are the respective concentration change of electrons and holes. Two material parameters, conductivity effective mass $m_{c e}^{*}, m_{c h}^{*}$ and mobility $\mu_{e}, \mu_{h}$ for electron and hole, respectively, differentiate the plasma dispersion effect in different materials.

In general, SOI modulators can be divided mainly in three different types: carrier depletion, carrier injection and, the less common, carrier accumulation modulators. The three most common parameters for describing the performances of silicon photonic phase shifters: propagation losses, modulation bandwidth and modulation efficiency.

Usually, the most common way to take advantage of the different types of silicon photonic modulators is the Mach-Zehnder interferometers (MZI), which converts the change of the phase shifts of two arms into the change of the MZI output amplitude via constructive or destructive interferences. The basic structure of doping regions in carrier-depletion phase shifter can be divided into three main categories: vertical, horizontal and interdigital PN junction.

In this work, we used a carrier depletion featuring a vertical p-n junction, which gives 
the best modulation efficiency to maximize the extinction ratio in a symmetrical Mach-Zehnder interferometer, featuring a broad optical bandwidth, that is discussed in the following sections.

\subsection{Design Passive Components}

In this section, the passive building block of the silicon modulator are briefly presented. The components are:

- Strip and rib waveguides

- Rib-to-strip transitions

- $90^{\circ}$ bends, both rip and strip

- 1x2 MMI

Waveguides are the building block of a photonic circuit. For this specific design, two different geometries of waveguides, strip and rib, have been used. In photonic integrated circuits, the first aspect that has to be taken into account is to ensure the single mode operation of the waveguides. This is mandatory to rule out the creation of higher order modes, which would degrade the optical signal clarity propagation through the photonic integrated circuit. Mainly, this aspect is obtained by controlling the width of the waveguide, because the thickness of the SOI is usually fixed and can't be changed. The other important aspect is to minimize, as much as possible, the propagation losses of the waveguides.

In the O-band, strip waveguides have a single mode behavior up to a width of $\sim 620$ $\mathrm{nm}$ for a thickness of $220 \mathrm{~nm}$ of SOI, as shown in Fig. 1.1(a). For this specific layout, a nominal width of $400 \mathrm{~nm}$ has been chosen which allows the bend radius to be reduced, lowering as a result the footprint of the entire chip.

This work requires the use of rib waveguides, which are essential for the implementation of a vertical pn junction in the Mach-Zehnder modulator's phase shifter. Similarly to strip waveguides, the designed rib waveguide has a thickness of $220 \mathrm{~nm}$, however, the edges of the waveguide are not fully etched, instead the etch depth is $70 \mathrm{~nm}$. The chosen width is $400 \mathrm{~nm}$, which ensure a single mode operation also in this case. However, as depicted by Fig. 1.1(b), the transition from single mode to 


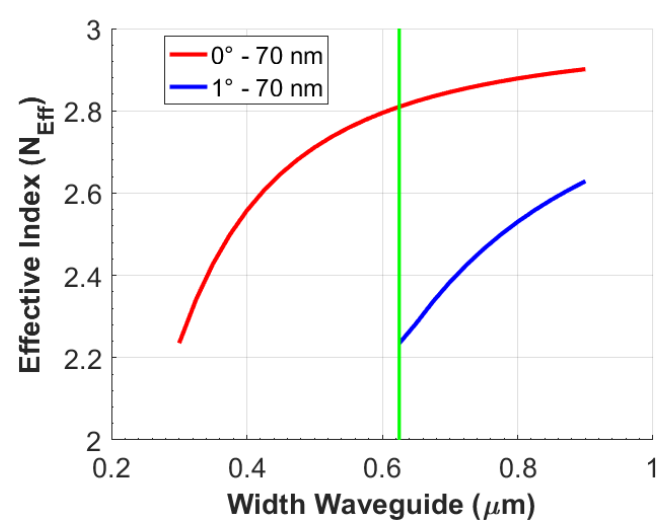

(a)

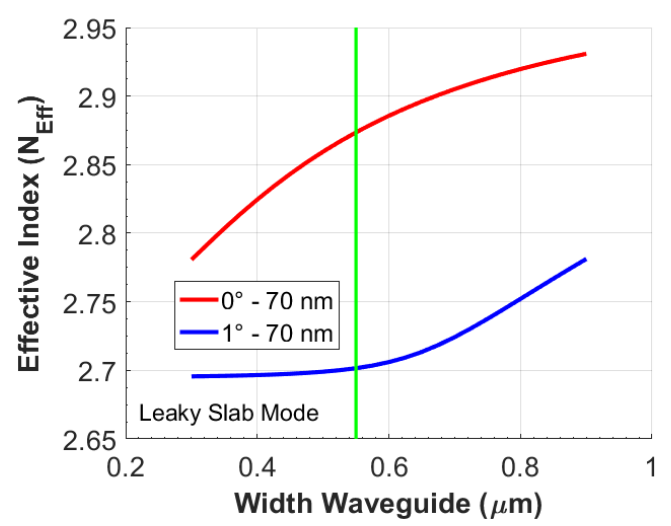

(b)

Figure 1.1: The graphs shows the variation of effective refractive index of a (a) strip waveguide and (b) rib waveguide at its width variation. For the strip waveguide the change in regime, from single mode to multimode is quite abrupt. On the other hand for the rib waveguide the transition is smoother. Therefore, the actual field distribution of the optical mode is required in order to better understand the real behavior of the waveguide, and its transition to the multi-mode regime.

multimode occurs width a lower width, due to the presence of the slab. So it's fundamental to have information over the field distribution in order to ensure the single mode operation of the waveguide. As it's shown in Fig. 1.2, at $400 \mathrm{~nm}$ the field distribution of the first order mode is mainly in the slab. However, it's important to underline the fact that this solution of the mode solver describes a non-guided mode, which ensures that the rib waveguide with a width of $400 \mathrm{~nm}$ is single mode. Due to the presence of both type of geometries, the use of rib-to-strip converters and viceversa is requested to ensure a smooth transition, minimizing the losses and maintaining the single mode behavior. They have been simulated using BPM and 3D-FDTD, in both direction of propagation, resulting in a length of $15 \mu \mathrm{m}$ for both direction and its losses can be considered negligible.

Subsequently, the $90^{\circ}$ bends have been optimized for both strip and rib geometries. In general, waveguide bends exhibit two main sources of losses: propagation losses due to sidewall roughness and other process/material dependent effects as well as bending losses which are inherent to the bend and radius itself. Conventional circular deep-etched $90^{\circ}$ turns at $1550 \mathrm{~nm}$ exhibit a well know $1 / r$ loss dependence which holds near $1310 \mathrm{~nm}$ wavelength. Low loss turns with radii as low as 1, 2 and $5 \mu \mathrm{m}$ 


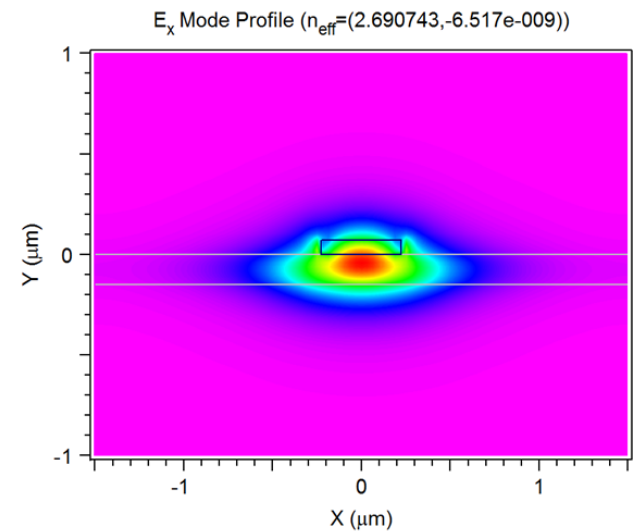

(a)

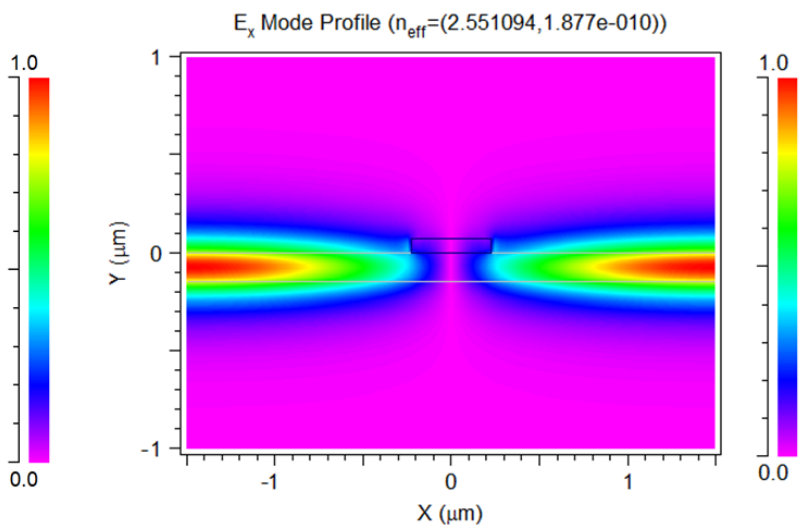

(b)

Figure 1.2: Field distribution at $400 \mathrm{~nm}$ in a rib waveguide for the (a) fundamental and (b) first order mode. One can observe that the field distribution of the first order mode is mainly in the slab, which describe a situation in which the mode is not supported. Therefore the waveguide is single mode at $400 \mathrm{~nm}$ width.

across the entire 1325-1550 $\mathrm{nm}$ range were demonstrated in a deep etched waveguide configuration, having propagation losses respectively of $0.086 \pm 0.005,0.013 \pm 0.005$, and $0 \pm 0.005 \mathrm{~dB} /$ bend [17]. However, between 1270 and $1325 \mathrm{~nm}$ the losses seem to increase reaching values of $0.05 \mathrm{~dB}$ for both 2 and $5 \mu \mathrm{m}$ radii and $>0.1 \mathrm{~dB}$ for $1 \mu \mathrm{m}$. It must be noted that these bends do not include any optimization and therefore may be subject to additional mode radiation at the transition between the bend output/input and the straight waveguide. One of the traditional ways is to compensate this by introducing an offset, shifting the centerline of the bent waveguide slightly to the center of the bend, compared with the centerline of the straight waveguide. The resulting offset is only a few nanometers and usually lies below the resolution limits of deep-UV steppers. In order to reach a compromise between loss and footprint we chose $10 \mu \mathrm{m}$ as a safe value for the radius of the strip waveguides operating within the $1270 \mathrm{~nm}-1330 \mathrm{~nm}$ wavelength range.

Rib shallow-etched waveguide bends suffer also from slab leakage losses, which are produced by the optical coupling to the lateral slab and they are highly dependent from slab geometry and radius, as shown in Fig. 1.3. Near 1550nm, it has been shown that the singular refractive index distribution of a standard rib waveguide 

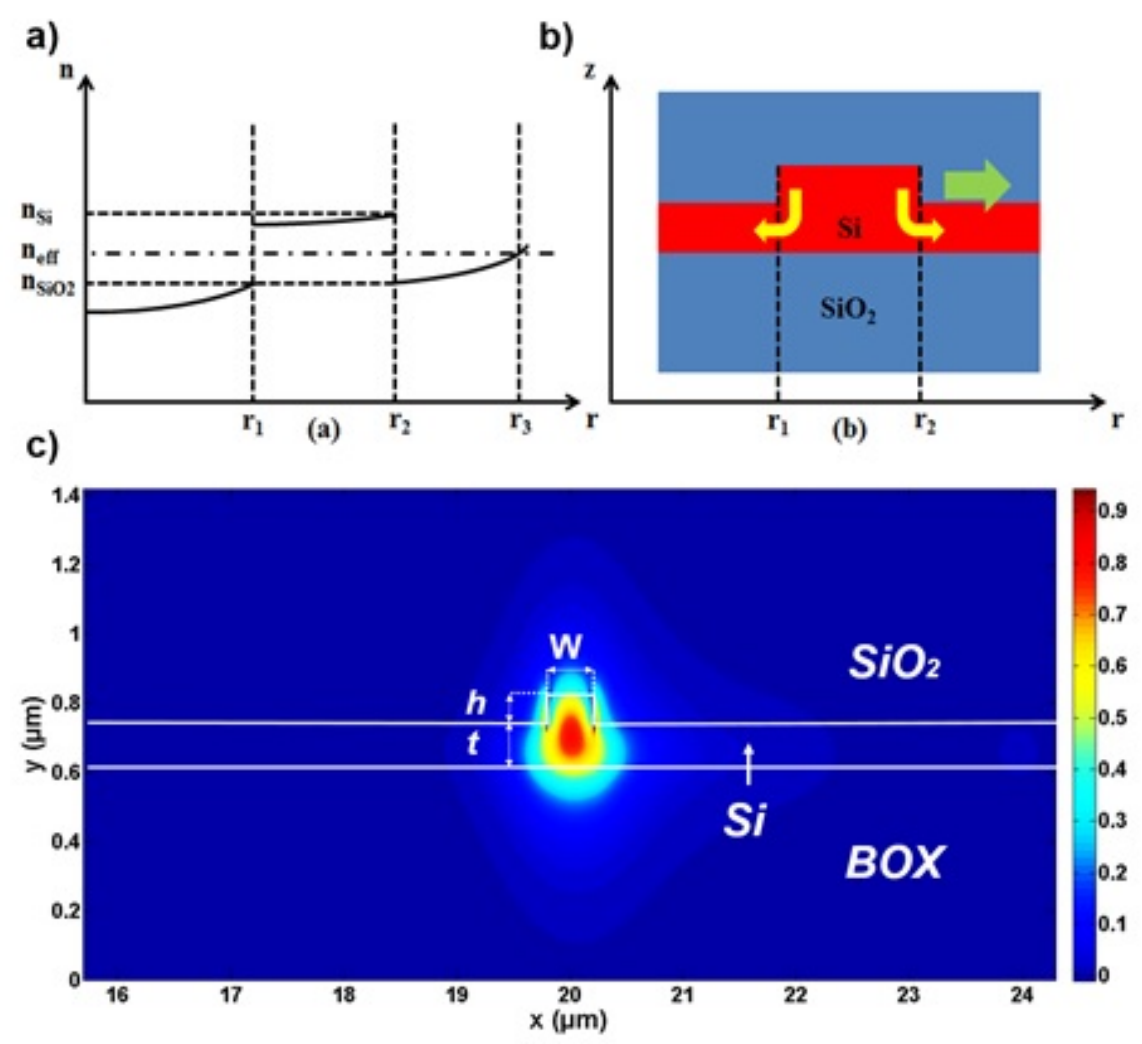

(a)

Figure 1.3: a) Equivalent refractive index profile (n) of a rib waveguide bend versus radius (r) and b) schematic representation of the sources of losses in a rib bend waveguide. Namely, bending losses (Green arrow pointing towards the outer part of the bend, higher $\mathrm{r}$ values) and leakage losses produced by coupling to the slab mode (yellow arrows). Mode profiles for c) $20 \mu \mathrm{m}$ radii. A pronunced modal asymmetry can be observed.

bend, associated with the presence of the slab, mitigates in some cases the bending losses in a certain range of bend radii and hence produces a local minimum loss region for small radii [17]. This allows rib bends radii to be only $25 \mu \mathrm{m}$ which with losses around $0.09 \mathrm{~dB}$. Ignoring these interesting fact shifts the next practical radius to around $500 \mu \mathrm{m}$ with the same losses. Following the same method and modelling tools, rib bends around near 1310nm were simulated showing a similar tendency, but shifted to larger radiis $(50 \mu \mathrm{m}-60 \mu \mathrm{m})$ with a pronounced local minimum, as shown in Fig. 1.4. Bends loss are prohibitive below $30 \mu \mathrm{m}$, but become quickly very low $(\sim 0.003 \mathrm{~dB})$ and comparable to strip bend losses for radii over $50 \mu \mathrm{m}$, which is the value that our specific design. 


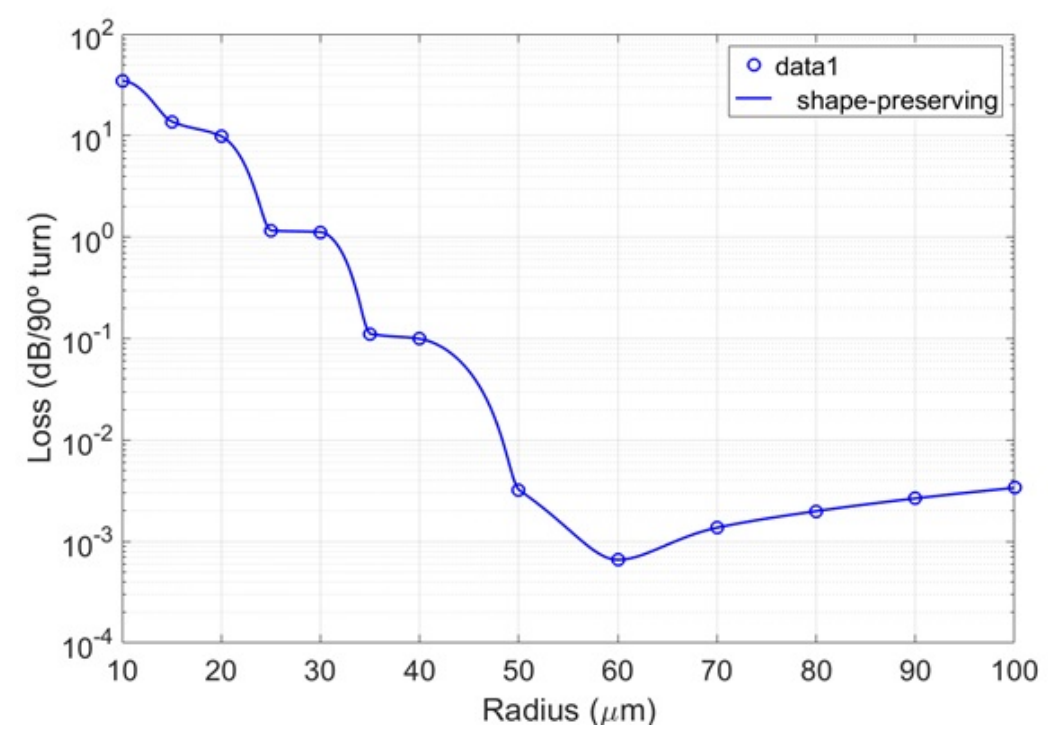

(a)

Figure 1.4: Shallow-etched bend loss vs radius; a local minimum can be observed when the radius is near $\sim 60 \mu \mathrm{m}$.

The last building block is the Multimode interference (MMI), which are commonly used in modern photonic devices and optoelectronics integrated circuits, thanks to their compactness, large bandwidth, overall low losses and robustness against fabrication variations. The designed MMIs feature a rib geometry, with an etch depth of $70 \mathrm{~nm}$, as in the case of the phase shifter. In order to fulfill compactness requirements and allow relaxed fabrication process tolerances, the dimensions $\left(L_{m m i}\right.$ $\left.* W_{m m i}\right)$ of the multimode section are $8.6 \mu \mathrm{m} * 2.5 \mu \mathrm{m}$. The device has been simulated using 3D-FDTD and BPM. Additionally, the tapers of the MMI have been engineered to maximize the ingoing and outgoing light from the multimode region, and are symmetric at the input and asymmetric at the output. The choice of using asymmetrical tapers is mandatory in order to avoid the presence of sharp corners on the device, reducing accordingly the losses caused by the roughness induced by the fabrication process. The overall losses of the symmetrical MMI are around $\sim 0.3-0.4$ $\mathrm{dB}$, with the desired 50:50 splitting ratio. 


\subsection{Phase shifter and modulator design}

The phase shifter (PS) and modulator design follows these steps:

1. Fabrication process simulation of the phase-shifter

2. DC simulations

3. Transient regime

4. Analytical design of the modulator

It is important to underline that the phase shifter fabrication process, DC simulations and transient responses have been simulated using ATHENA and ATLAS, respectively process and device simulator by Silvaco. Instead, the theoretical response of the modulator have been simulated using an analytical model of a MZM [18].

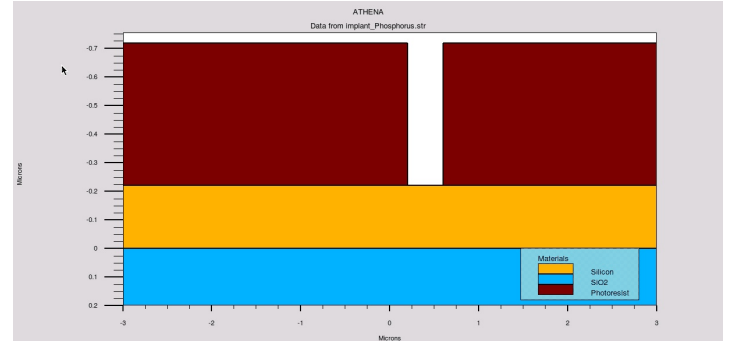

(a)

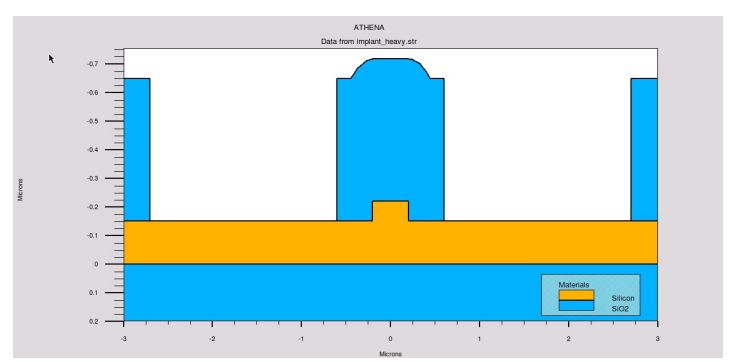

(c)

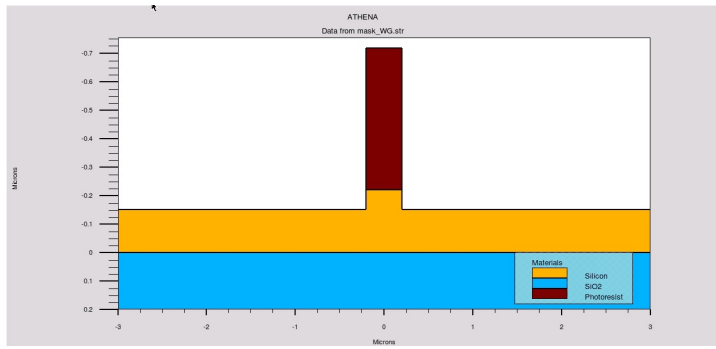

(b)

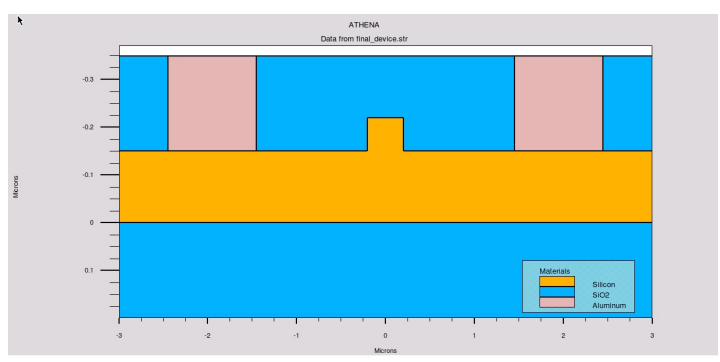

(d)

Figure 1.5: The pictures summarises the mainly steps in the simulation of the phase shifter fabrication process. (a) Implantation of the dopants for the creation of the pn junction. (b) Definition of the waveguide. (c) Implantation of the doped regions. (d) Oxide windows opening and final metallization. The pictures have been obtained from Silvaco (Appendix B).

The fabrication process simulation is briefly summarized in Fig. 1.5. (a) Starting 
from a $220 \mathrm{~nm}$ thick silicon slab on top of a $3 \mu \mathrm{m}$, the process starts with the definition of $3 \mu \mathrm{m}$ wide pn junction, through the creation of an opening of the same width, followed by the dopants implantation. (b) Then, the $400 \mathrm{~nm}$ waveguide is created with a depth-etch of $70 \mathrm{~nm}$. (c) The heavily doped regions are then created and the dopant electrical activation is performed through Rapid Thermal Annealing (RTA). It is actually in this phase of the fabrication that the width of the pn junction is set. (d) The fabrication simulations is then ended creating the oxide windows opening and performing the final metallization.

The number of design parameters that have to be taken into account when designing a phase shifter can be manifold. However, due to geometry constraint, such as the width of the waveguide and depth of the etch step, target performance (high-speed, low driving voltage) and secondary optimization, i.e. the energy of implantation, the variables have been reduced to two: doses of the doping and width of the pn junction.

The implantation energies have been set to: $30 \mathrm{keV}$ for $\mathrm{p}++/ \mathrm{n}++$ regions, $100 \mathrm{keV}$ (p doping) and $150 \mathrm{keV}$ (n doping). The low energies for the heavy doped regions is required sto ensure ohmic contacts at the interface between the silicon and aluminium. The $\mathrm{p}$ and $\mathrm{n}$ regions values have been optimized in order to allow for a clear separation of the two regions, maximizing as a result the efficiency of the phase shifter as a result.

The target speed of the modulator requires the use of high doping levels with concentrations of the order of $\sim 10^{18}$. As a result, after careful studies, 8 different combinations of p-n implantations, to achieve the desired concentrations, have been taken into account for the realization of the phase shifter, and they are summarized in Table 1.1 .

The PS performance is first evaluated under static conditions with reverse bias spanning from $V_{i n}=0 \mathrm{~V}$ to $V_{\text {fin }}=-5 \mathrm{~V}$. When a reverse bias is applied to the pn junction, the space charge region width increases as the carriers are depleted from the junction. This variation in the concentration of carriers induces a change in the refractive index of the silicon [16] as a function of the applied reverse bias voltage. This produces a change in the effective index of the optical mode, which results from the overlap between the optical mode and real refractive index distributions of the 


\begin{tabular}{c|c}
\hline $\begin{array}{c}\text { p-type } \\
\left(\mathrm{cm}^{-2}\right)\end{array}$ & $\begin{array}{c}\text { n-type } \\
\left(\mathrm{cm}^{-2}\right)\end{array}$ \\
\hline \hline $1.5 \cdot 10^{13}$ & $2.5 \cdot 10^{13}$ \\
$1 \cdot 10^{13}$ & $2 \cdot 10^{13}$ \\
$2 \cdot 10^{13}$ & $3 \cdot 10^{13}$ \\
$4 \cdot 10^{13}$ & $5 \cdot 10^{13}$ \\
$6 \cdot 10^{13}$ & $8 \cdot 10^{13}$ \\
$7 \cdot 10^{13}$ & $5 \cdot 10^{13}$ \\
$5 \cdot 10^{12}$ & $7 \cdot 10^{12}$ \\
$9 \cdot 10^{12}$ & $1 \cdot 10^{13}$ \\
\hline \hline
\end{tabular}

Table 1.1: This table displays the doses that have been taken into account for the design of the phase shifter.

waveguide.

These changes affect both the real and imaginary components of the effective index, determining changes in the phase and propagation losses, respectively, of the PS. An example of this behaviour can be observed in Fig. 1.6.

When the reverse bias increases the effective index has a positive increase when

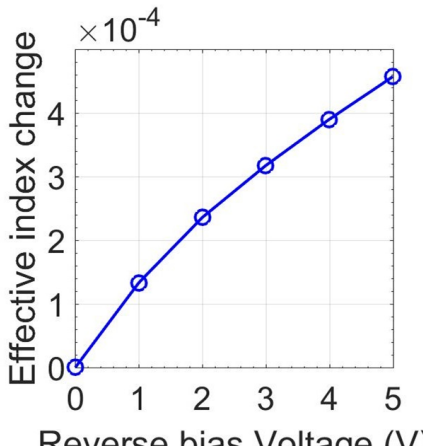

Reverse bias Voltage (V)

(a)

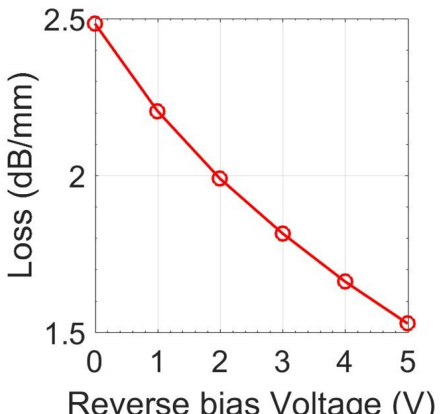

(b)

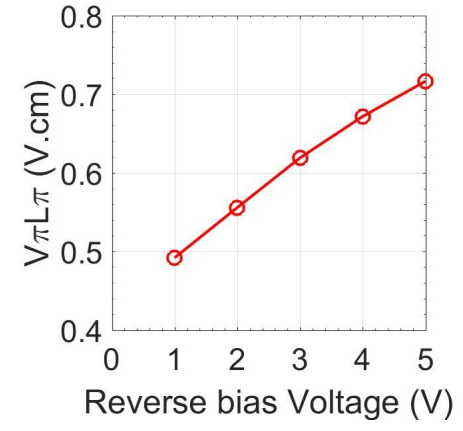

(c)

Figure 1.6: These figures exemplify (a) effective refractive index change, (b) propagation losses and (c) FOM trend at the increase of the reverse bias voltage for a standard carrier depletion plasma phase shifter with doping of $4 \cdot 10^{13}-5 \cdot 10^{13}$.

compared to the unbiased regime, which translates into a phase increase. Moreover, the losses decreases at the rise of the biased voltage. On the other hand, the FOM 


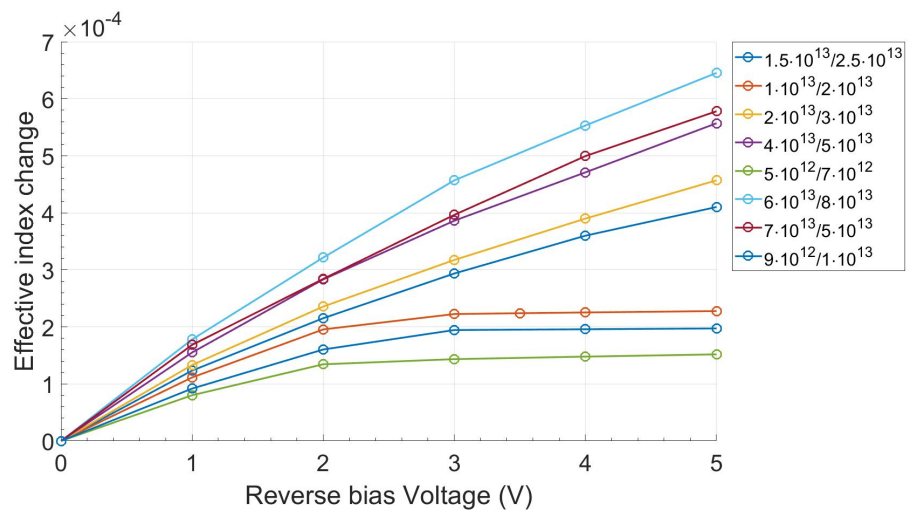

(a)

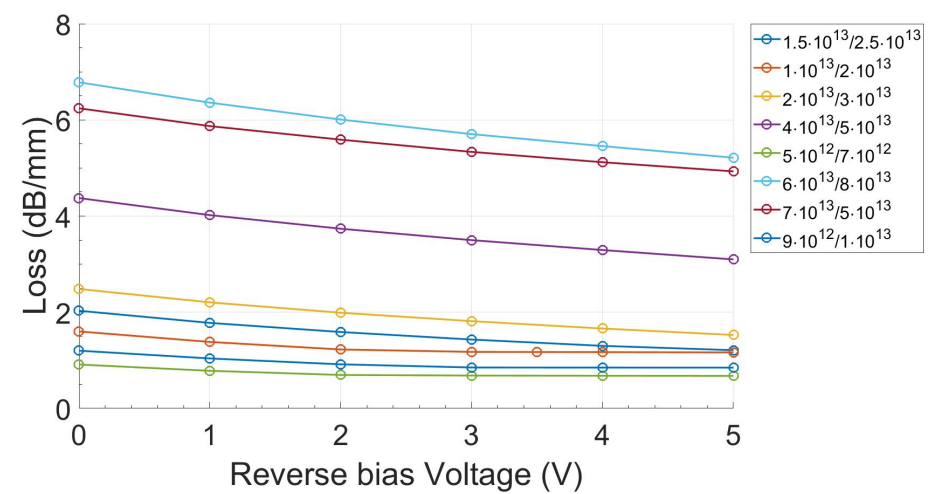

(b)

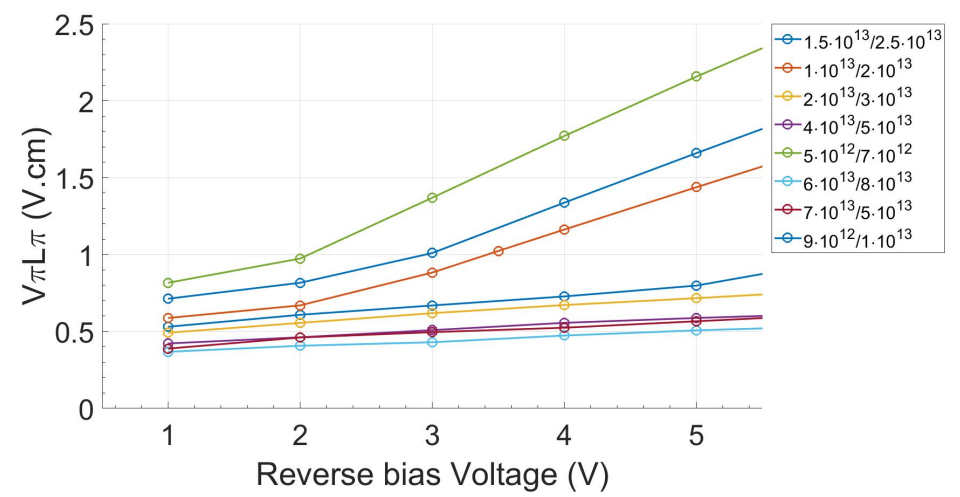

(c)

Figure 1.7: These graphs summarize (a) effective refractive index change, (b) propagation losses and (c) FOM at increased reverse bias voltage of the phase shifter for the eight implantation doses under investigation, which are summarized in Table 1.1 .

denoted as the $V_{\pi} L_{\pi}$ decreases as the reverse bias voltage increases.

This kind of analysis has been carried out for all eight the above mentioned doping 
doses and the results are summarized in Fig. 1.7. It is possible to observe that, for each one of the quantities under investigations, an increase in doping doses determines a different effect.

Figure 1.7 (a) shows that as, the doping doses increases, the effective index change is more prominent when a reverse bias voltage is applied. This trend can be ascribed to by the higher level of carriers and the subsequent strength of the electric field induced by the higher doping concentration levels. Interestingly, for lower doses the $\Delta n_{\text {eff }}$ reaches a plateau for $\mathrm{V}<-3$, which has to be ascribed to the inability of further extract carriers from the pn junction due to the low doping concentrations. Indeed, the depletion width is larger for lower doping concentrations, and as the voltage increases, the pn depletion is enlarged accordingly and quickly reaches the $\mathrm{Si} / \mathrm{SiO}_{2}$ interfaces, resulting in a saturation effect. This effect is well described in [19].

These low doping concentrations have also a direct effect over the losses in the phase shifter. As depicted in Fig. 1.7(b), the losses increase when increasing the implantation doses: from as low as below $1 \mathrm{~dB} / \mathrm{mm}\left(5 \cdot 10^{12}-7 \cdot 10^{12}\right)$ up to $\sim 7 \mathrm{~dB} / \mathrm{mm}\left(6 \cdot 10^{13}\right.$ $\left.-8 \cdot 10^{13}\right)$. Moreover, as expected, one can clearly observe that the losses are reduced as the reverse bias voltage is increased, due the decrease free-carrier concentration. Similarly to the refractive index change, the efficiency $\left(V_{\pi} L_{\pi}\right)$ gets better (Fig. $1.7(\mathrm{c}))$, with values as low as $0.3-0.4 \mathrm{~V} \cdot \mathrm{cm}$, when the doping doses are increased. It is interesting to observe that the FOM's values increase slightly when the reverse biased is increased. However, for the three lower doping doses, the efficiency worsen as the reverse bias increases, due to their inability of further extract carrier when the doping is relatively low.

Other important quantities, that have to be taken into account for the design, are the time responses $\left(\tau_{\text {fall }}\right.$ and $\left.\tau_{\text {rise }}\right)$ of the pn junction when a reverse bias voltage is applied. Transient simulation have been performed in order to estimate the available bandwidth at our disposal for the final MZM. Table 1.2 summarises the results for all 8 implantations under investigation for a width of the pn junction of $1.6 \mu \mathrm{m}$. They show a clear trend: higher implantation doses determines lower $\tau_{\text {fall }}$ and $\tau_{\text {rise }}$, which are mandatory in order to achieve the desired high speed performances. On the other hand, as shown previously, higher doping also increases the propagation 


\begin{tabular}{c|c|c}
\hline \hline $\begin{array}{c}\text { Doping Doses } \\
(\mathrm{p}-\mathrm{n})\left(\mathrm{cm}^{-2}\right)\end{array}$ & $\begin{array}{c}\tau_{\text {rise }} \\
(\mathrm{ps})\end{array}$ & $\begin{array}{c}\tau_{\text {fall }} \\
(\mathrm{ps})\end{array}$ \\
\hline \hline $1.5 \cdot 10^{13}-2.5 \cdot 10^{13}$ & 62 & 66 \\
$1 \cdot 10^{13}-2 \cdot 10^{13}$ & 83 & 93 \\
$2 \cdot 10^{13}-3 \cdot 10^{13}$ & 42 & 54 \\
$4 \cdot 10^{13}-5 \cdot 10^{13}$ & 30 & 42 \\
$6 \cdot 10^{13}-8 \cdot 10^{13}$ & 29 & 39 \\
$7 \cdot 10^{13}-5 \cdot 10^{13}$ & 29 & 38 \\
$5 \cdot 10^{12}-7 \cdot 10^{12}$ & 72 & 123 \\
$9 \cdot 10^{12}-1 \cdot 10^{13}$ & 60 & 81 \\
\hline \hline
\end{tabular}

Table 1.2: This table summarizes the doses that have been taken into account for the design of the phase shifter.

losses of the phase shifter, so a trade-off between overall efficiency and losses has to be found.

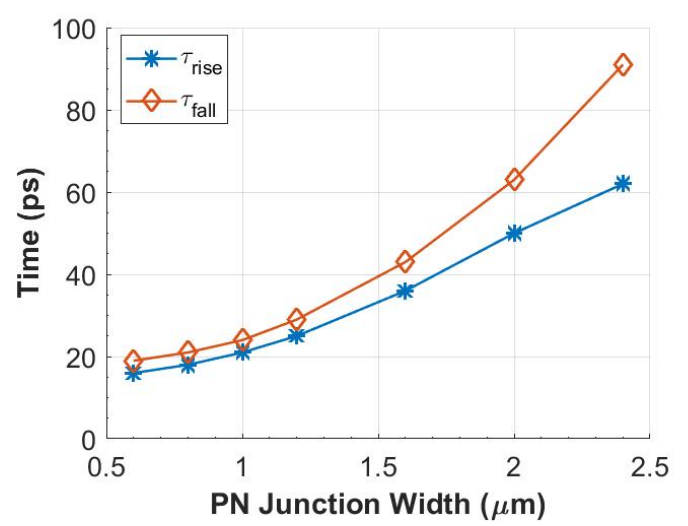

(a)

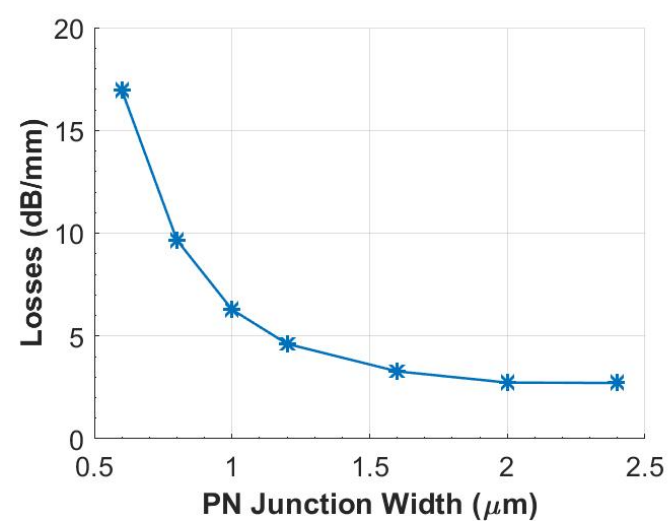

(b)

Figure 1.8: These graphs show (a) the dependance of the rise and fall time of the phase-shifter and (b) propagation losses at the increase of the pn junction width.

The last important variable that has to be properly designed is the width of the pn junction, which has significant effects on the performance of the phase shifter. An example can be observed in Fig. 1.8, which shows that the phase shifter's speed 
and losses are greatly dependant on the geometry. A reduction in width of the pn junction decreases significantly $\tau_{\text {fall }}$ and $\tau_{\text {rise }}$, allowing in this way the design and realization of high speed SOI modulators. This change in geometry has the drawback to increase also the propagation losses of the phase shifter, which increase as the pn junction width decreases. This trend is caused by the increased overlap of the optical mode with the highly doped regions. As a consequence, a trade-off between the two quantities is required and this aspect is fundamental for the proper design of the desired modulator, especially taking into consideration the trade-off required between losses and efficiency that is highly dependant upon the implantation doses.
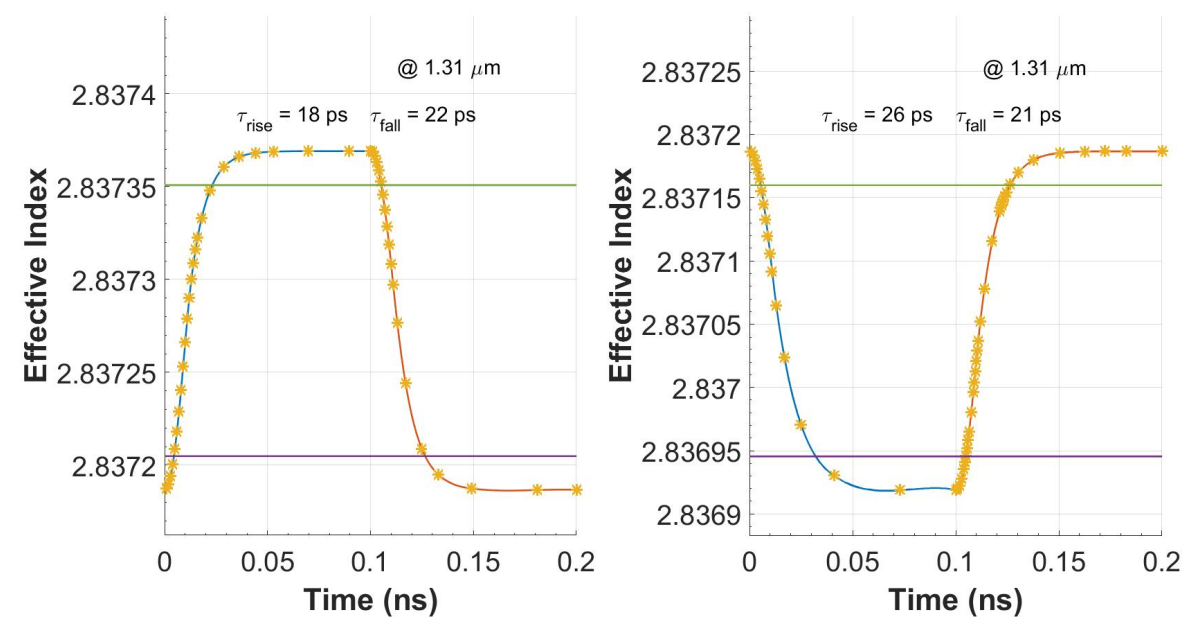

Figure 1.9: The graphs show the transient responses of the phase shifter in the two different regimes at which they are driven for their implementation in the MZM.

Therefore, for the design of a high-speed and highly efficient modulator the first two main parameters that have to be determined are the doping doses and the pn junction width. Following the previous discussion, for this specific layout, it has been chosen to use the $4 \cdot 10^{13}-5 \cdot 10^{13}$ doping dose and set the width of the phase shifter to $1.2 \mu \mathrm{m}$. Moreover, in order to reduce as much as possible the overall device footprint, the MZM will be operating in a push-pull configuration, where in each one of the arm of the Mach-Zehnder Interferometer a phase shifter is present, and each one operates within different voltage ranges. The device have been optimized for operations at $V_{D C}$ set to $-1.6 \mathrm{~V}$ for the "on-state", thus biasing the phase shifters of the MZM in a reverse bias regime, and applying voltage swings of $V_{p p} 2.5 \mathrm{~V}$. Op- 
eratively, the two phase shifters have been biased in two different regions, namely $-2.85 \mathrm{~V}$ and $-0.35 \mathrm{~V}$, and then the $2.5 \mathrm{~V}$ voltage swings will be applied. Figure 1.9 represents the transient responses of the phase shifter in the two different regimes. A fast transient response can be observed, on the order of $20 \mathrm{ps}$, is achieved. Noticeably, these rise and fall times ensure the capability of the designed MZM to be able to operate at $25 \mathrm{GHz}[20,21]$.

Yet, it is important to point out that the different operating biases determine distinct values of rise and fall times. At lower biases the transient response of the phase shifter is faster (Fig. 1.9(a)) then to operations below the pn junction threshold regime or biases between to $0 \mathrm{~V}$ and $+0.8 \mathrm{~V}$ (Fig. 1.9(b)) [22]. Nonetheless, a higher depletion voltage decreases the efficiency of the phase shifter, which is measured in terms of $\Delta \mathrm{n}_{\text {eff }}$. This trade-off between speed and efficiency has to be carefully studied and analysed in order to allow for a precise design of the modulator.

Therefore, the asymmetrical behaviour of the MZM has profound effect in the final step of the design of the modulator, where the length of the MZM has to be determined. For this reason, a final tuning of the modulator has to be done, taking into account the specifications, in terms of losses and extinction ratio of the MZM, which are mainly determined by the platforms and applications in which the device will be utilized. For the design under investigation, a final length of $930 \mu \mathrm{m}$ has been chosen.

To better understand the various aspects of the MZM design, a more extensive analysis has been carried out to compare the selected specifications with other possible variations, namely with other doping profiles. They have been compared to the inverted of $\mathrm{p}$ and n-type implantation doses.

For the desired application, a target extinction ratio of $3.5 \mathrm{~dB}$ is set. As a result, the required length to achieve the specified value is calculated, together with the resulting IL. Table 1.3 and 1.4 summarize the results, respectively for the DC and RF cases. From table 1.3 , one can observe how a lower doping profile requires a longer phase shifter to be able to reach the desired ER, in accordance with the simulations previously presented. Additionally, a lower doping concentration determines a reduction in the overall IL of the modulator. However, as expected, an higher doping profile induces a better FOM and a smaller footprint of the device. Thus, a 


\begin{tabular}{c|c|c|c}
\hline \hline $\begin{array}{c}\text { Doping Doses } \\
(\mathrm{p}-\mathrm{n})\left(\mathrm{cm}^{-2}\right)\end{array}$ & $\begin{array}{c}L_{\text {mod }}(D C)(\mathrm{mm}) \\
(\mathrm{ER}=3.5 \mathrm{~dB})\end{array}$ & $\begin{array}{c}\text { IL @ DC }(\mathrm{dB}) \\
(\mathrm{ER}=3.5 \mathrm{~dB})\end{array}$ & $\begin{array}{c}\mathrm{FOM} \\
(\mathrm{V} \cdot \mathrm{cm})\end{array}$ \\
\hline \hline $6 \cdot 10^{13}-8 \cdot 10^{13}$ & 0.644 & 4.62 & 0.22 \\
$4 \cdot 10^{13}-5 \cdot 10^{13}$ & 0.772 & 3.75 & 0.26 \\
\hline \hline
\end{tabular}

Table 1.3: This table summarizes the specifications in DC for the two doses under investigation.

\begin{tabular}{c|c|c|c}
\hline \hline $\begin{array}{c}\text { Doping Doses } \\
(\mathrm{p}-\mathrm{n})\left(\mathrm{cm}^{-2}\right)\end{array}$ & $\begin{array}{c}L_{\text {mod }}(\sim 18 \mathrm{GHz})(\mathrm{mm}) \\
(\mathrm{ER}=3.5 \mathrm{~dB})\end{array}$ & $\begin{array}{c}\text { IL @ } \sim 18 \mathrm{GHz}(\mathrm{dB}) \\
(\mathrm{ER}=3.5 \mathrm{~dB})\end{array}$ & $\begin{array}{c}\text { FOM } \\
(\mathrm{V} \cdot \mathrm{cm})\end{array}$ \\
\hline \hline $6 \cdot 10^{13}-8 \cdot 10^{13}$ & 0.839 & 6.12 & 0.26 \\
$4 \cdot 10^{13}-5 \cdot 10^{13}$ & 1.034 & 5.17 & 0.33 \\
\hline \hline
\end{tabular}

Table 1.4: This table summarizes the specifications at high frequency for the two doses under investigation.

trade-off, expecially between insertion losses and efficiency of the modulator has to be taken into account.

It is worth noting that the performance at higher frequencies becomes worse. Indeed, in order to obtain the desired ER, a longer phase shifter is needed, and this increase is more pronounced when the doping doses are decreased. Quantitatively, this increase can be estimated in roughly $\sim 30-35 \%$. This aspect can be physically justified by the fact that, for carrier depletion modulator, the efficiency decreases as the driving frequency increases, which consequently decreases the $\Delta n_{E f f}$ produced by the phase shifter itself. Therefore, this lower performance have to be compensated by increasing the total length of the modulator.

Also, tables 1.5 and 1.6 show how an increase of the desired ER affects the footprint and IL of the devices, both in DC and RF regimes. It is expected that increasing the target ER gives, as a result, longer phase-shifter, to which are associated also higher insertion losses.

Overall, these tables shows again the complexity of designing a phase shifter and the numerous parameters that have to be taken into account for its design for a specific application. Mainly, the E/O bandwidth, modulation efficiency and insertion losses have to be considered since they are directly linked with the speed of the photonic 


\begin{tabular}{c|c|c|c}
\hline \hline Doping Doses & $\mathrm{ER}=3.5 \mathrm{~dB}$ & $\mathrm{ER}=5.0 \mathrm{~dB}$ & $\mathrm{ER}=7.0 \mathrm{~dB}$ \\
$(\mathrm{p}-\mathrm{n})\left(\mathrm{cm}^{-2}\right)$ & $\begin{array}{c}L_{\text {mod }}(\mathrm{DC})(\mathrm{mm}) \\
\mathrm{IL}(\mathrm{dB})\end{array}$ & $\begin{array}{c}L_{\text {mod }}(\mathrm{DC})(\mathrm{mm}) \\
\text { IL }(\mathrm{dB})\end{array}$ & $\begin{array}{c}L_{\text {mod }}(\mathrm{DC})(\mathrm{mm}) \\
\mathrm{IL}(\mathrm{dB})\end{array}$ \\
\hline \hline $6 \cdot 10^{13}-8 \cdot 10^{13}$ & $0.644 / 4.62$ & $0.749 / 5.38$ & $0.855 / 6.13$ \\
$4 \cdot 10^{13}-5 \cdot 10^{13}$ & $0.772 / 3.75$ & $0.899 / 4.37$ & $1.026 / 4.98$ \\
\hline \hline
\end{tabular}

Table 1.5: This table shows how an increase in desired ER affects the footprint and insertion losses of the phase-shifter (in DC).

\begin{tabular}{c|c|c|c}
\hline \hline Doping Doses & $\mathrm{ER}=3.5 \mathrm{~dB}$ & $\mathrm{ER}=5.0 \mathrm{~dB}$ & $\mathrm{ER}=7.0 \mathrm{~dB}$ \\
$(\mathrm{p}-\mathrm{n})\left(\mathrm{cm}^{-2}\right)$ & $L_{\text {mod }}(\mathrm{RF})(\mathrm{mm})$ & $L_{\text {mod }}(\mathrm{RF})(\mathrm{mm})$ & $L_{\text {mod }}(\mathrm{RF})(\mathrm{mm})$ \\
& $\mathrm{IL}(\mathrm{dB})$ & $\mathrm{IL}(\mathrm{dB})$ & $\mathrm{IL}(\mathrm{dB})$ \\
\hline \hline $6 \cdot 10^{13}-8 \cdot 10^{13}$ & $0.839 / 6.12$ & $0.980 / 7.17$ & $1.136 / 8.32$ \\
$4 \cdot 10^{13}-5 \cdot 10^{13}$ & $1.034 / 5.17$ & $1.208 / 6.07$ & $1.397 / 7.05$ \\
\hline \hline
\end{tabular}

Table 1.6: This table shows how an increase in desired ER affects the footprint and insertion losses of the phase-shifter (in RF).

integrated circuits, power consumption and its optical power budget.

\subsection{Fabrication and Measurements}

The fabrication of the modulator has been entirely carried out in the AMS CMOS foundry, with $248 \mathrm{~nm}$ DUV lithography. The recipe used by AMS followed closely the optimised implantation doses targeted in the previous section, $4 \cdot 10^{13}-5 \cdot 10^{13}$ $\mathrm{cm}^{-2}$ for the $\mathrm{p}$ and $\mathrm{n}$-type regions respectively. According to our simulations, these implantation doses will ensure the best performance in terms of modulation efficiency footprint-bandwidth trade-off. A brief description is giving below of the steps the foundry took for the fabrication of the phaseshifter, which are also sketched in Fig. 1.10 for completeness. Firstly, phosphorus $(\mathrm{P})$ is implanted with an energy of 150 $\mathrm{keV}$ and a dose reaching $5 \cdot 10^{13} \mathrm{~cm}^{-2}$ to form the n-type region. For the p-type region, BF3 $(\mathrm{BF} 2+)$, which has been preferred over Boron $(\mathrm{B})$ due to its shallow implantation profile [23], is implanted with an energy of $100 \mathrm{keV}$ and a dose reaching the desired $4 \cdot 10^{13} \mathrm{~cm}^{-2}$ (Fig. 1.10(a)). 
a)

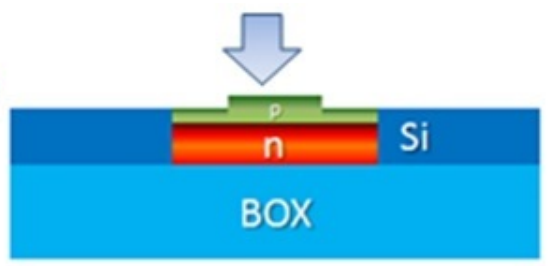

Vertical pn junction definition

c)

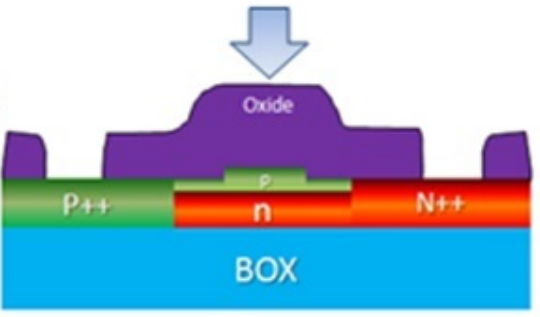

Oxide windows openings

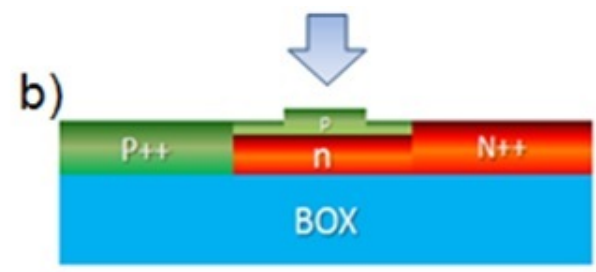

P++ and N++ implantations

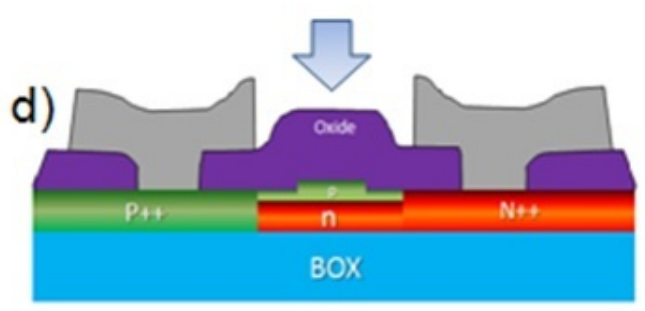

Before metallization

Figure 1.10: Process flow of the fabricated silicon modulator. (a) Implantation of the p-n junction doses, having as target the doses of $4 \cdot 10^{13}-5 \cdot 10^{13} \mathrm{~cm}^{-2}$. (b) Shallow implantation of the heavy doped regions $(\mathrm{p}++/ \mathrm{n}++)$ for the creation of ohmic contact between the SOI region and tungsten vias. (c) Deposition of the oxide and subsequent creation of the opening required for the vias. (d) Deposition of the vias and metal layer.

Specifically, the realization of the $1.2 \mu \mathrm{m}$-wide junction makes use of only one lithography mask. This approach is beneficial for two main reasons: the reduction of the costs relative to using only one mask rather than two and the minimization of potential alignment errors associated with using additional masks. Misalignment of the mask can be a severe issue, thus it is of great importance to minimize it, and the use of a single mask allows it. Physically, a misalignment in the masks would create an out-of-specification pn junction, which can give unexpected results especially in terms of bandwidth and propagation losses. Thus, it is of paramount importance to be able to correctly and reliably create the correct geometry for the pn junction.

Subsequently, the $\mathrm{p}++$ and $\mathrm{n}++$ regions were both formed with a high dose (1e15 $\left.\mathrm{cm}^{-} 2\right)$ and shallow $(20 \mathrm{keV})$ implant, in order to ensure good ohmic contact between the tungsten vias and the SOI region, where the phase-shifter is located (Fig. 1.10(b)). Ohmic contacts are required for delivering efficiently the RF signal to the phaseshifter, allowing the modulation of the $\mathrm{CW}$ signal coming from the input of the modulator. Finally, a layer of oxide is deposited on top of the newly formed 
a)

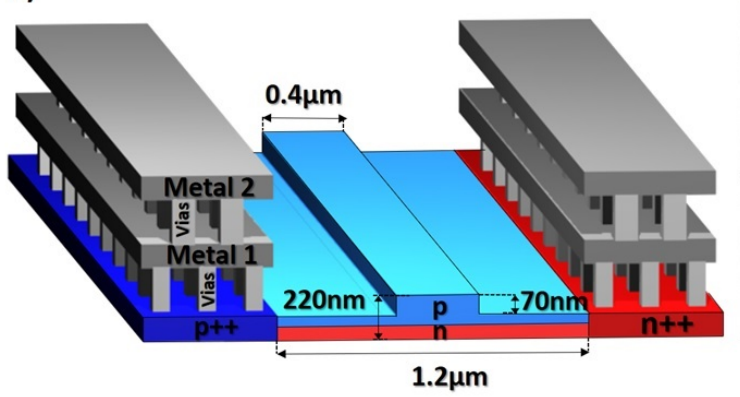

b)

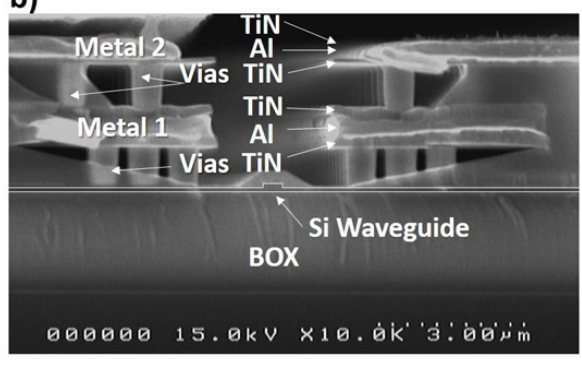

Figure 1.11: (a) 3D layout of phaseshifter. (b) SEM image of the cross-section of the fabricate modulator.

phaseshifter, and windows on its sides are opened, allowing for deposition of tungsten vias and metal layer. The metal layer is composed of a multilayered structure composed of an aluminum sheet sandwiched between two thin layers $(\sim 40-50 \mathrm{~nm})$ of titanium nitride, as depicted in Fig. 1.11(b). This final step is repeated twice before finalizing the modulator, as showed in the 3D layout of Fig. 1.11(a).

Figure 1.12 describes the generic setup used to estimate DC and RF response of the device. Transverse electric (TE) polarized light emitted by an external cavity laser is injected into the modulator via grating couplers, which are the ideal devices for in-lab testing of such type of component, and optimized through the use of a polarization controller. In this specific case, the grating coupler have been designed for coupling at $1310 \mathrm{~nm}$ [24] and for 10.9 coupling angle in air. The coupling unit has an insertion losses of $\sim 4.5-5 \mathrm{~dB}$ and this design parameters: a period $(\Lambda)$ of $665 \mathrm{~nm}$ and filling factor $(\mathrm{FF})$ of 0.6. Unfortunately, AMS's BEOL is different from the one for which the grating couplers were designed, thus the efficiency is not optimal and it was not feasible to further optimize them due to time constraints at the foundry during the project duration.

The fabricated device (Fig. 1.13(a)) has been tested firstly in DC, at different voltages, and the results can be observed in Fig. 1.13(b). It's possible to observed the high ER $(>12 \mathrm{~dB})$ at $1310 \mathrm{~nm}$ that we have been able to obtain for moderate voltage swing $(\sim 2.5 \mathrm{~V})$, and this proves the high efficiency of the phaseshifter we designed. Moreover, the relatively high 3-dB bandwidth of the modulator of approx- 


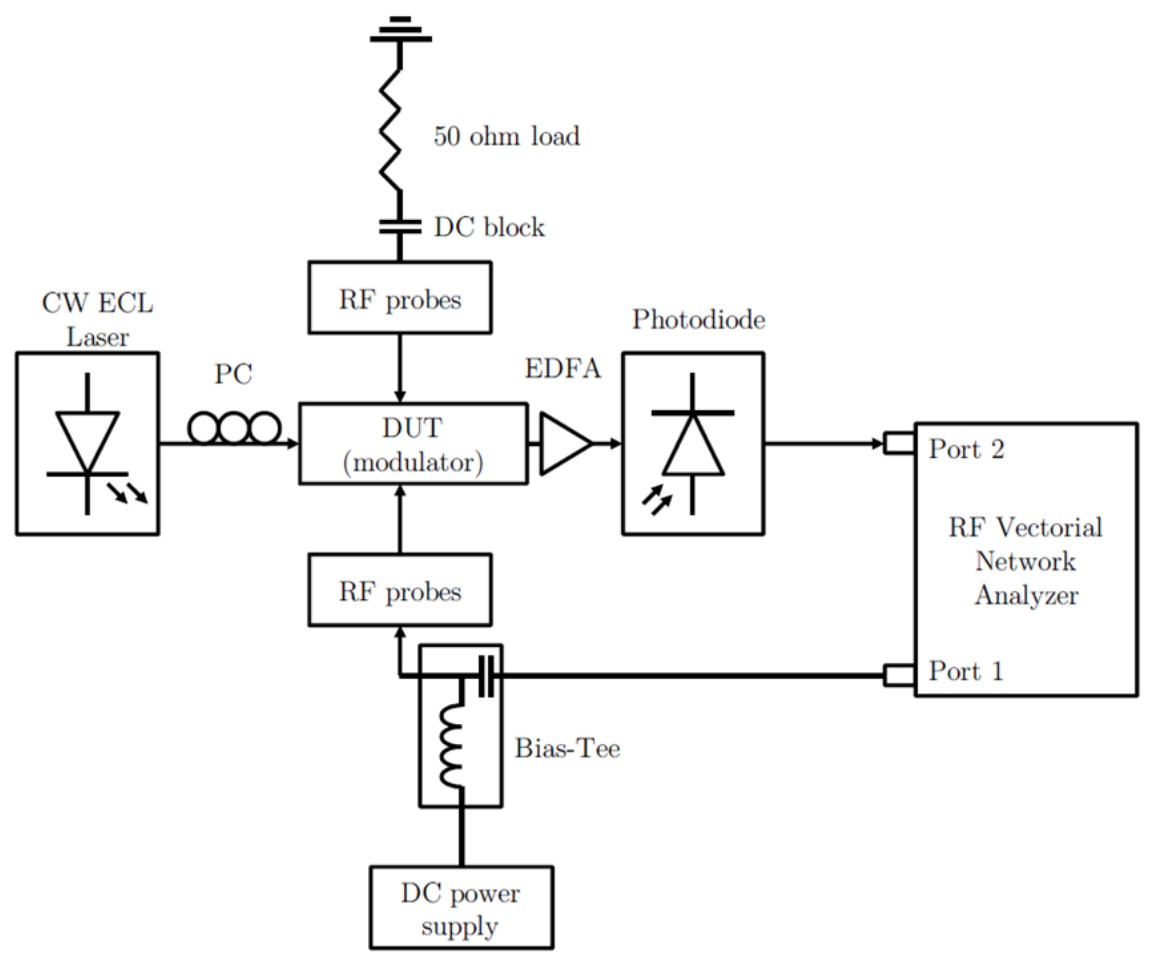

(a)

Figure 1.12: Schematic of the DC and RF setup used for the characterization of the device.

a)
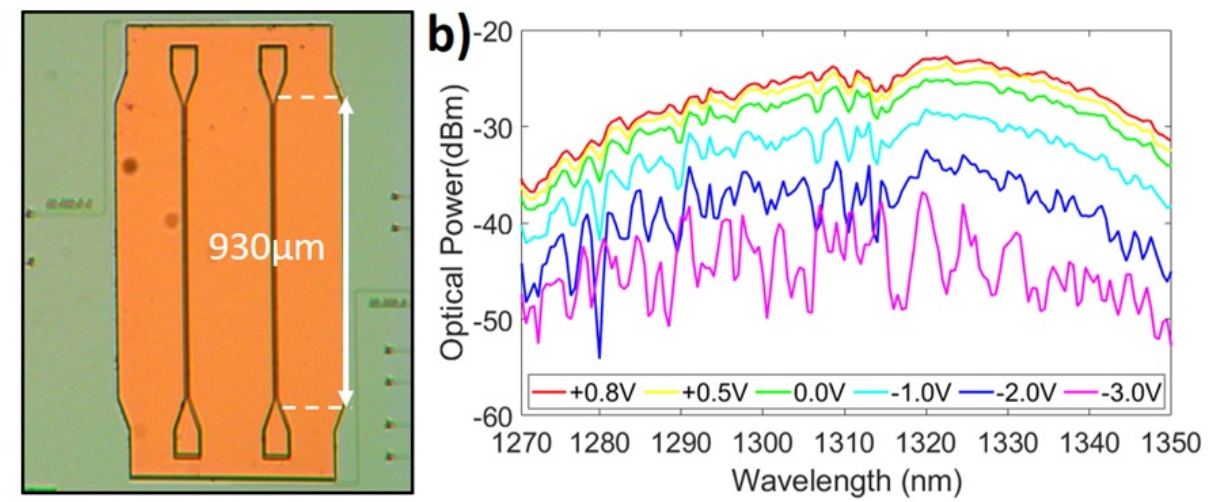

Figure 1.13: (a) Fabricated device (b) DC response of the MZM from $+0.8 \mathrm{~V}$ to $-3 \mathrm{~V}$.

imately 20-30 nm, due to the use of grating couplers, shows the capability of the device to operate, not only at $1310 \mathrm{~nm}$, but also in DWDM applications. To maximise the MZM optical bandwidth, a wavelength-independant light-coupling (such as butt-coupling) method could be readily be used, making the device compatible with CWDM applications. 
Unfortunately, the spectra show an high level of Fabry-Perot resonances, which, according to our investigations, are caused by the high sidewall roughness of the full-etched waveguides used for routing and other building block inside the chip. To solve this issue, it's foreseen to use rib waveguides for the routing to minimize as much as possible this undesirable effect.

For the AC characterization, the modulators have been characterized using a bit pattern generator (model SHF BPG 44E) delivering a non-return-to-zero pseudorandom bit sequence (NRZ PRBS-1) of length $2^{31}-1$ using different biases, namely $1.6 V_{p p}, 2.3 V_{p p}$ and $3.2 V_{p p}$ applied in a differential manner 1.14 . The symmetric nature of the modulators makes their operation less wavelength dependent than their asymmetric counterparts, making them ideal candidates for broad-band application such as CWDM and DWDM.

For the designed modulator of $930 \mu \mathrm{m}$ long phase-shifter a $\pi$-phase shift is achieved at a $4 \mathrm{~V}$ variation, leading to a measured $\mathrm{V} \pi \mathrm{L}$ of $0.35-0.40 \mathrm{~V} . \mathrm{cm}$. This measured FOM positions our modulator among the state-of-the-art compared to other devices working in the "O-band" [25, 26]. The insertion losses produced by the $0.93 \mathrm{~mm}$-long active (doped) arms of the Mach-Zehnder is approximately of $4.6 \mathrm{~dB}$ which is caused by the high levels of doping concentration.

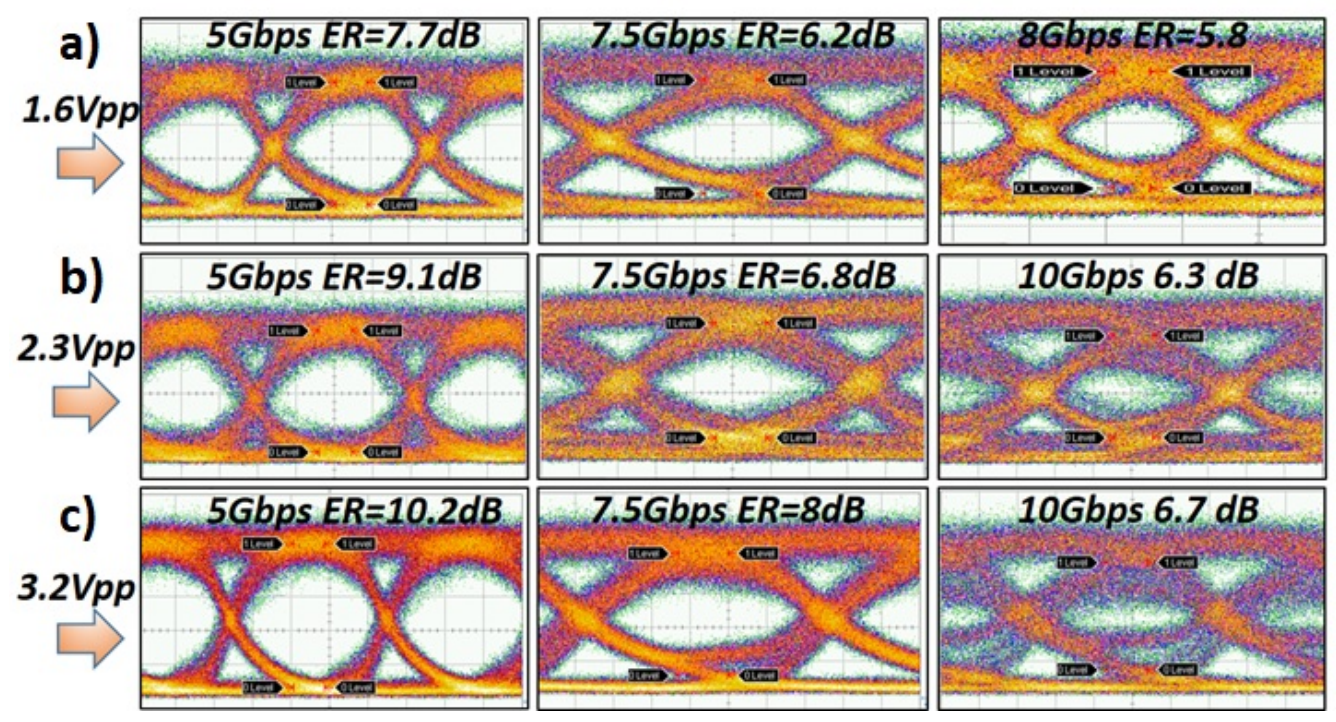

Figure 1.14: RF response of the MZM at different biases (a) $1.6 \mathrm{~V}$ (b) $2.3 \mathrm{~V}$ (c) $3.2 \mathrm{~V}$ and different speeds. It is to notice that increasing the speed of the modulator the ER decreases (for constant voltage) and increasing the voltage biases, at a constant speed, the ER increases. 
However, only a data transmission up to $10 \mathrm{~Gb} / \mathrm{s}$ has been demonstrated with enough extinction ratio to allow direct detection of the modulated signal, using relative low differential drive voltages.

In order to further investigate the issue, the modulator RF response has been characterized on a dedicated high speed test bench. The Scattering (S-)parameters. The S parameters (S21, S11, S12, S22) have been extracted with a Vectorial network analyzer (Agilent) with measurement capabilities up to $67 \mathrm{GHz}$. A careful calibration procedure has been used to eliminate potential distortion and losses arising from the RF external circuit (cables, connectors, etc) circuit to extract only the travelling wave electrode response. We show here only three modulators measurements which are representative of all the measurements performed over four samples. Sparameters as well as impedance values for varying electrode length are extracted from the latter and are shown in Figure 1.15. As can be observed, although the impedances of the travelling wave electrodes are relatively well matched to $50 \Omega$ (confirmed by the S11 measurements which are below -10 dB) across the bandwidth of interest $(0-25 \mathrm{GHz})$ in all three cases (with some more pronounced variations for an electrode length of $1.25 \mathrm{~mm}$ ) the $\mathrm{S} 21$ response shows a rapid decrease, showing at most a $7.6 \mathrm{GHz}$ bandwidth for the shortest travelling wave electrode ( $\mathrm{TL}=0.55$ $\mathrm{mm}$ ). To find out the cause of this limited bandwidth, the microwave losses were calculated for different electrode types and length as shown in Figure 1.16. In all cases, the electrodes exhibit high losses, with slightly higher values for the dual drive configuration. The results of three identical electrodes on the same sample show a good uniformity giving us confidence in the quality of the measurement and yield of the fabrication process. In order to understand to what extent these microwave losses affect the bandwidth and RF modulation efficiency, we take the example of the $1.25 \mathrm{~mm}$ electrode. The best case scenario (orange curve, Fig. 1.16) shows a microwave loss of $11.3 \mathrm{~dB}$ at $5 \mathrm{GHz}$ and $17.6 \mathrm{~dB}$ at $20 \mathrm{GHz}$. Assuming a perfect impedance match $(50 \Omega)$ between the input probe and transmission line, a full 1.8 $V_{p p}$ voltage amplitude is transferred to the electrode. Based on the calculated attenuation values, after a travelling half way across the electrodes, the electrical signal is already attenuated by $5.65 \mathrm{~dB}$ at $5 \mathrm{GHz}$ (respectively, $8.8 \mathrm{~dB}$ at $20 \mathrm{GHz}$ ). The input voltage is halved $(0.9 \mathrm{~V})$ at $5 \mathrm{GHz}$ and then attenuated by a factor of $3(0.65 \mathrm{~V})$ at $20 \mathrm{GHz}$. At the end of the transmission line (end of the modulator), the effective 
driving voltage is $0.5 V_{p p}$ at $5 \mathrm{GHz}$ and $0.25 V_{p p}$ at $20 \mathrm{GHz}$. From these results it is clear that improving the microwave loss is key to reach the desired bandwidth.

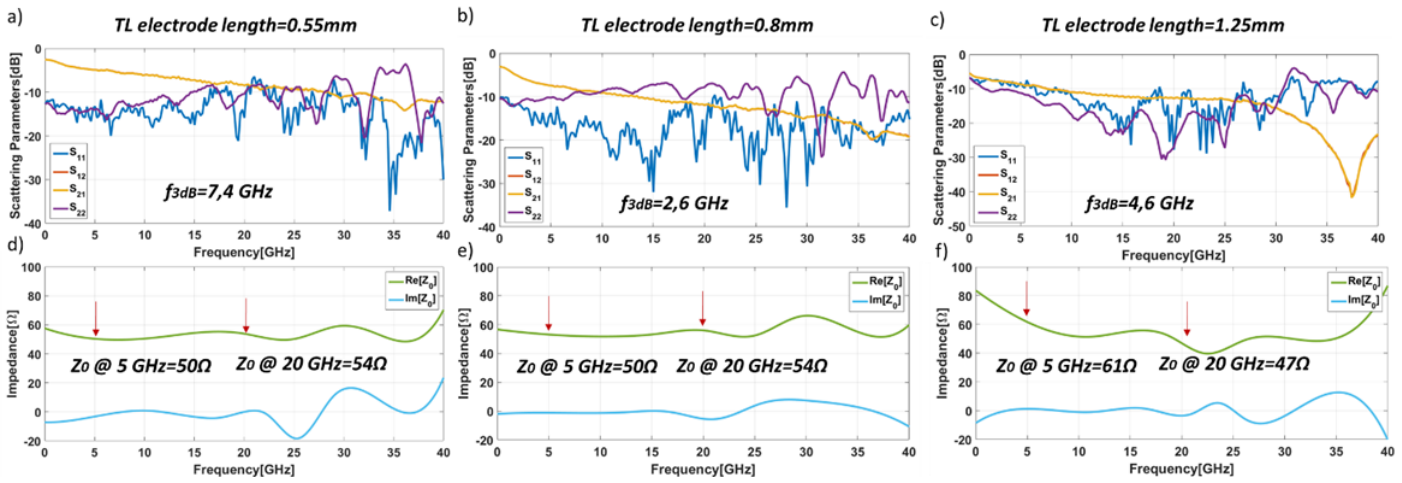

Figure 1.15: (a), (b), (c) S-parameters and (d), (e), (f) impedance of the transmission line for varying electrode length, $0.55 \mathrm{~mm}, 0.8 \mathrm{~mm}$, and $1.25 \mathrm{~mm}$, respectively.

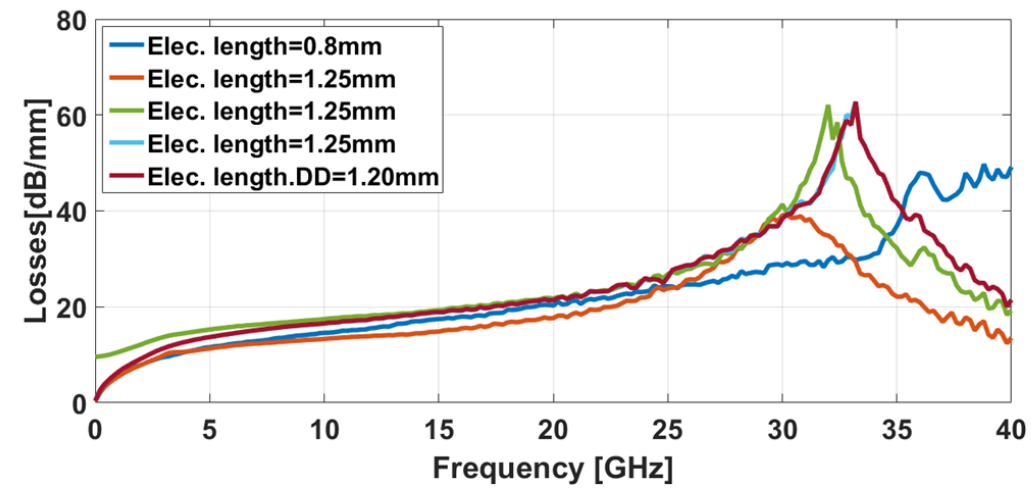

Figure 1.16: Microwave loss for varying single electrode test structure length and type. DD refers to the dual drive electrodes used in the designed modulator.

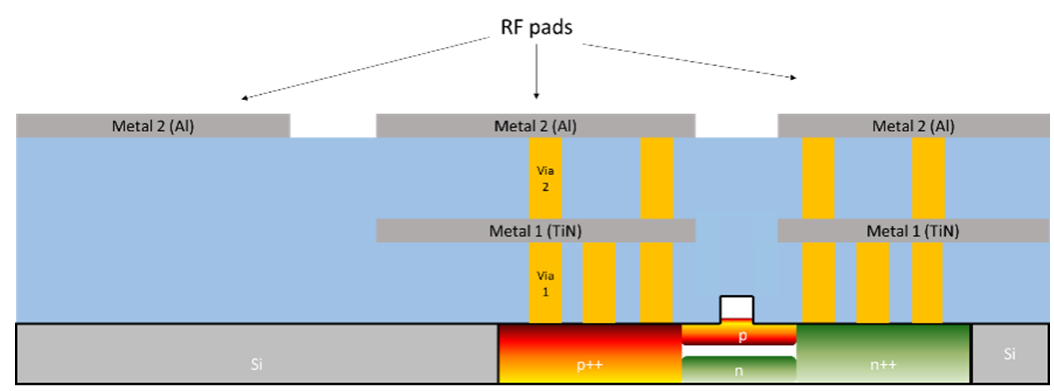

Figure 1.17: Metal layer stack configuration. 
To address this issue, we plan to change the metal stack layer configuration where TiN is used as metal 1 (Fig. 1.17). TiN is highly resistive compared to aluminum and we retain it to be the main cause of microwave losses.

Simulations (Fig. 1.18(a)) also show the limited bandwidth of the electro-optical device, and consequently also the bit-rate that we are able to allocate.

Further improvements of the metals stack and of the TW electrodes will in the future increase the bandwidth of the overall modulator (Fig. 1.18(b)), enabling data transmissions up to $25 \mathrm{~Gb} / \mathrm{s}-30 \mathrm{~Gb} / \mathrm{s}$, lying closer to the intrinsic bandwidth of the pn junction.

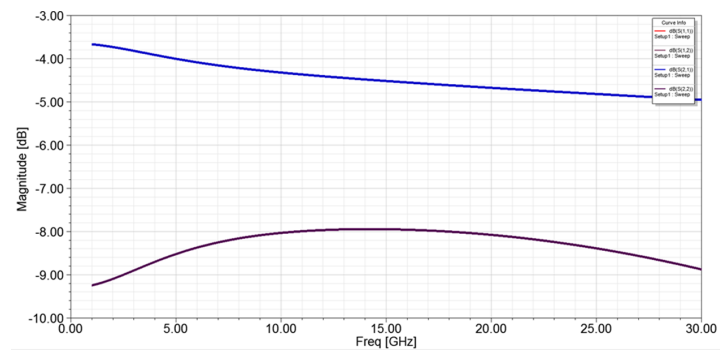

(a)

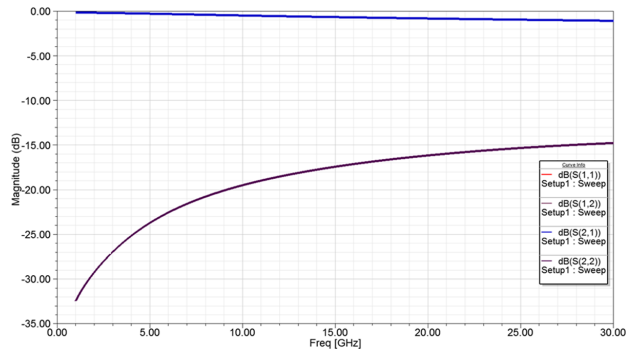

(b)

Figure 1.18: S-parameter simulations of (a) the actual fabricated layer-stack-up of the MZM and (b) optimized electrodes for future fabrication runs.

\subsection{Conclusions}

In conclusion, our design and experimental results demonstrate the potential for highly efficient, low-power MZM modulators implementing the plasma dispersion effect in carrier depletion regime in silicon optical modulators. For this design the main limitations come from the intrinsic electro-optic bandwidth of the modulator and the its trade-off with the propagation losses of the phase-shifter.

Overall, carrier depletion based silicon optical modulators remain one of the strongest candidate for photonic integrated circuits in data centres and HPC applications. Novel design features are continuously being introduced which are yielding on going improvements in the device performance. 


\section{Bibliography}

[1] Ansheng Liu, Richard Jones, Ling Liao, Dean Samara-Rubio, Doron Rubin, Oded Cohen, Remus Nicolaescu, and Mario Paniccia. A high-speed silicon optical modulator based on a metal-oxide-semiconductor capacitor. Nature, 427(6975):615-618, 2004.

[2] Xiaojun Xie, Qiugui Zhou, Erik Norberg, Matt Jacob-Mitos, Yaojia Chen, Anand Ramaswamy, Gregory Fish, John E Bowers, Joe Campbell, and Andreas Beling. Heterogeneously integrated waveguide-coupled photodiodes on soi with $12 \mathrm{dbm}$ output power at $40 \mathrm{ghz}$. In Optical Fiber Communication Conference, pages Th5B-7. Optical Society of America, 2015.

[3] Jayavardhana Gubbi, Rajkumar Buyya, Slaven Marusic, and Marimuthu Palaniswami. Internet of things (iot): A vision, architectural elements, and future directions. Future generation computer systems, 29(7):1645-1660, 2013.

[4] Alfred Zimmermann, Michael Pretz, Gertrud Zimmermann, Donald G Firesmith, Ilia Petrov, and Eman El-Sheikh. Towards service-oriented enterprise architectures for big data applications in the cloud. In 2013 17th IEEE International Enterprise Distributed Object Computing Conference Workshops, pages 130-135. IEEE, 2013.

[5] Ghavam G Shahidi. Soi technology for the ghz era. IBM journal of Research and Development, 46(2.3):121-131, 2002.

[6] Tsung-Yang Liow, Kah-Wee Ang, Qing Fang, Jun-Feng Song, Yong-Zhong Xiong, Ming-Bin Yu, Guo-Qiang Lo, and Dim-Lee Kwong. Silicon modulators and germanium photodetectors on soi: monolithic integration, compatibility, and performance optimization. IEEE Journal of Selected Topics in Quantum Electronics, 16(1):307-315, 2009.

[7] James F Buckwalter, Xuezhe Zheng, Guoliang Li, Kannan Raj, and Ashok V Krishnamoorthy. A monolithic 25 -gb/s transceiver with photonic ring modulators and ge detectors in a 130-nm cmos soi process. IEEE Journal of Solid-State Circuits, 47(6):1309-1322, 2012. 
[8] Andrew Huang, Cary Gunn, Guo-Liang Li, Yi Liang, Sina Mirsaidi, A Narashimha, and Thierry Pinguet. A $10 \mathrm{gb} / \mathrm{s}$ photonic modulator and wdm mux/demux integrated with electronics in $0.13 / \mathrm{spl} \mathrm{mu/m}$ soi cmos. In 2006 IEEE International Solid State Circuits Conference-Digest of Technical Papers, pages 922-929. IEEE, 2006.

[9] Alan F Benner, Petar K Pepeljugoski, and Renato J Recio. A roadmap to $100 \mathrm{~g}$ ethernet at the enterprise data center. IEEE Communications Magazine, 45(11):10-17, 2007.

[10] Lars Dittmann, Anna Manolova Fagertun, Valerija Kamchevska, Michael Galili, L Oxenlove, S Ruepp, and M Berger. A roadmap for evolving towards optical intra-data-center networks. In ECOC 2016; 42nd European Conference on Optical Communication, pages 1-3. VDE, 2016.

[11] Roger R Schmidt, Christian Belady, Alan Classen, Tom Davidson, Magnus Herrlin, Shiomo Novotny, and Rebecca Perry. Evolution of data center environmental guidelines. ASHRAE Transactions, 110(1), 2004.

[12] Hwaiyu Geng. Data center handbook. John Wiley \& Sons, 2014.

[13] Tao Chu, Hirohito Yamada, Satomi Ishida, and Yasuhiko Arakawa. Compact $1 \times \mathrm{n}$ thermo-optic switches based on silicon photonic wire waveguides. Optics Express, 13(25):10109-10114, 2005.

[14] Álvaro Rosa, Ana Gutiérrez, Antoine Brimont, Amadeu Griol, and Pablo Sanchis. High performace silicon $2 \times 2$ optical switch based on a thermo-optically tunable multimode interference coupler and efficient electrodes. Optics express, 24(1):191-198, 2016.

[15] Jacek Gosciniak, Sergey I Bozhevolnyi, Thomas B Andersen, Valentyn S Volkov, Jakob Kjelstrup-Hansen, Laurent Markey, and Alain Dereux. Thermo-optic control of dielectric-loaded plasmonic waveguide components. Optics express, 18(2):1207-1216, 2010.

[16] RICHARDA Soref and BRIANR Bennett. Electrooptical effects in silicon. IEEE journal of quantum electronics, 23(1):123-129, 1987. 
[17] Antoine Brimont, Xuan Hu, Sébastien Cueff, Pedro Rojo Romeo, Guillaume Saint Girons, Amadeu Griol, Andrea Zanzi, Pablo Sanchis, and Régis Orobtchouk. Low-loss and compact silicon rib waveguide bends. IEEE Photonics Technology Letters, 28(3):299-302, 2015.

[18] Bahaa EA Saleh and Malvin Carl Teich. Fundamentals of photonics. john Wiley \& sons, 2019.

[19] Andrea Zanzi, Christos Vagionas, Amadeu Griol, Alvaro Rosa, Sergio Lechago, Miltiadis Moralis-Pegios, Konstantinos Vyrsokinos, Nikos Pleros, Jochen Kraft, Victor Sidorov, et al. Alignment tolerant, low voltage, 0.23 v. cm, push-pull silicon photonic switches based on a vertical pn junction. Optics express, 27(22):32409-32426, 2019.

[20] Ling Liao, Dean Samara-Rubio, Michael Morse, Ansheng Liu, Dexter Hodge, Doron Rubin, Ulrich D Keil, and Thorkild Franck. High speed silicon machzehnder modulator. Optics express, 13(8):3129-3135, 2005.

[21] FY Gardes, A Brimont, P Sanchis, G Rasigade, D Marris-Morini, L O'Faolain, F Dong, JM Fedeli, Pieter Dumon, L Vivien, et al. High-speed modulation of a compact silicon ring resonator based on a reverse-biased pn diode. Optics express, 17(24):21986-21991, 2009.

[22] Hong C Nguyen, Yuya Sakai, Mizuki Shinkawa, Norihiro Ishikura, and Toshihiko Baba. Photonic crystal silicon optical modulators: carrier-injection and depletion at $10 \mathrm{gb} / \mathrm{s}$. IEEE Journal of Quantum Electronics, 48(2):210-220, 2011.

[23] TS Chao. Introduction to semiconductor manufacturing technology. SPIE PRESS, 2001.

[24] Roberto Larrea, Ana Maria Gutierrez, and Pablo Sanchis. Design method for high performance grating couplers in photonic integrated circuits. Optical and Quantum Electronics, 50(9):341, 2018.

[25] Matthew Streshinsky, Ran Ding, Yang Liu, Ari Novack, Yisu Yang, Yangjin Ma, Xiaoguang Tu, Edward Koh Sing Chee, Andy Eu-Jin Lim, Patrick Guo-Qiang 
Lo, et al. Low power $50 \mathrm{gb} / \mathrm{s}$ silicon traveling wave mach-zehnder modulator near $1300 \mathrm{~nm}$. Optics express, 21(25):30350-30357, 2013.

[26] Diego Perez-Galacho, Charles Baudot, Tifenn Hirtzlin, Sonia Messaoudène, Nathalie Vulliet, Paul Crozat, Frederic Boeuf, Laurent Vivien, and Delphine Marris-Morini. Low voltage $25 \mathrm{gbps}$ silicon mach-zehnder modulator in the oband. Optics express, 25(10):11217-11222, 2017. 


\section{Chapter 2}

\section{Asymmetrical MMI}

This chapter presents the design, characterization of a novel asymmetrical multimode interference device for arbitrarily power splitting ratio, and its implementation in an electro-optical modulator, where it has been used to achieve high tunability of the device in terms of extinction ratio and propagation losses.

\subsection{Background and design}

Silicon-on insulator (SOI) is one of the most promising platforms to achieve dense integration of photonic devices at low cost, owing to its high-index contrast and compatibility with mature complementary metal-oxide semiconductor (CMOS) fabrication process [1, 2, 3]. Multimode interference (MMI) couplers one of the fundamental building block of photonic integrated circuits [4, 5]. MMIs, unlike other similar devices such as directional couplers, have several serious advantages when directly compared to other similar devices, including reduced footprint, broadband bandwidth and robust fabrication tolerances. As a result, their implementation in PIC as power splitters is really attractive [6]. They are commonly used as power splitter in Mach-Zehnder interferometers [7], splitters and combiners of various arbitrary splitting ratios [8], discriminators [9] and $90^{\circ}$ hybrids for coherent receivers applications [10].

The asymmetrical multi-mode interference (A-MMI) design has been carried out using 2D and 3D finite difference time domain (FDTD) method. The 2D simulations 
have been used to perform a preliminary analysis and set the coarse dimensions of the MMI. Subsequently, the 3D simulations allowed us to properly design and optimise the MMI sub-components parameters, namely: the multi-mode cavity dimensions (denoted as $W_{M M I}$ and $L_{M M I}$ in Fig. 2.1) and output taper geometry, for optimal coupling efficiency [11, 12].

The MMI working principle is mainly based on the concept of self-imaging, which is a property of multi-mode waveguides. In the latter, a propagating field profile of a given optical mode is reproduced periodically in a single or multiples images along the direction of propagation. The cavity is the core component because it is where the self-imaging occurs. Straight forwardly, $W_{M M I}$ and $L_{M M I}$ are the first two parameters to be determined. They are related by the following analytical expression (Eq. 2.1) [13, 14, 15]:

$$
L_{c}=\frac{\pi}{\beta_{0}-\beta_{1}}=\frac{4}{3} n \frac{W_{e q}^{2}}{\lambda}
$$

where $\beta_{0}$ and $\beta_{1}$ are the propagation constant of the first two modes, respectively, $W_{e q}$ is the approximated width, $\lambda$ is the central operating length and $n$ is an integer number.

The chosen width for the MMI is $W_{M M I}=3 \mu \mathrm{m}$, associated with a shallow etch

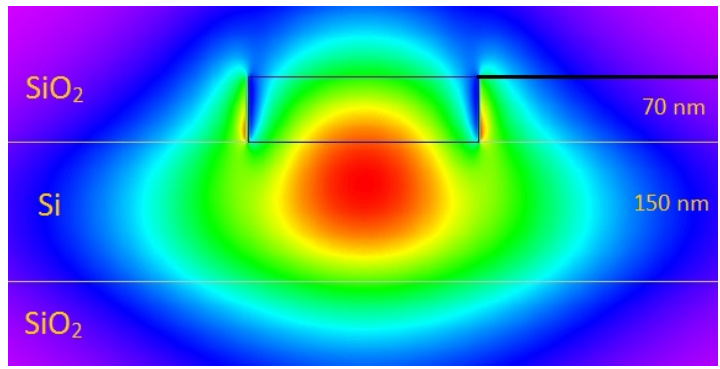

(a)

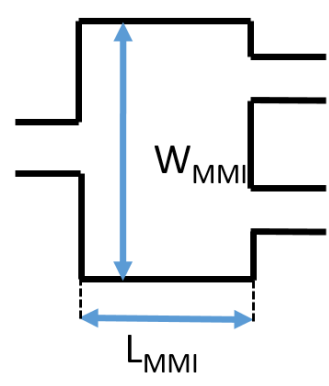

(b)

Figure 2.1: (a) Vertical cross-section of the waveguide. (b) Schematic and geometry of a standard MMI.

depth of $70 \mathrm{~nm}$ (leaving a $150 \mathrm{~nm}$ thick slab) (Fig. 2.1) yields $L_{M M I}=10 \mu \mathrm{m}$. These dimensions have been chosen carefully in order to find a trade-off between potential fabrication process deviations and device compactness.

The near infra-red light is injected into the multimode region through a $0.450 \mu \mathrm{m}$ 
wide waveguide and then tapered to reach a width of $0.85 \mu \mathrm{m}$. The two tapered output waveguides are precisely positioned at the first two folded images at $\pm W_{M M I} / 4$. The tapers of the A-MMI are engineered to maximize the ingoing and outgoing light from the multimode region, and are symmetric and asymmetric at the input and outputs, respectively (Fig. 2.2(a)). They have been modeled using 3D-FDTD simulations (Fig. 2.2(b)), and the optimized values for $\Delta_{1}$ and $\Delta_{2}$ are respectively $0.2 \mu \mathrm{m}$ and $0.525 \mu \mathrm{m}$. The lengths of the input and output tapers are $5 \mu \mathrm{m}$ and 15 $\mu \mathrm{m}$.

It has been decided to use asymmetric tapers at the outputs to avoid the presence of sharp corners on the device, reducing, as a result, the losses caused by the sidewall roughness as a result of the fabrication process. Moreover, this layout enables the coupling between the multi-mode region and the output waveguides to be maximized. In contrast with conventional symmetric 1x2 MMI featuring 50:50 power splitting ratios, our asymmetric MMI can achieve variable output splitting ratios through breaking the symmetry of the cavity The arbitrary power splitting ratios are obtained in the following manner: using as a starting vertex the connection point between the input taper and the multi-mode region, the cavity has been cut using the angle $(\theta)$ as a variable of reference as depicted in Fig. 2.2(a). The increment value of $\theta$, associated with an increasing removed area from the cavity, determines different values of the weaker output power ratio, denoted by $\mathrm{R}$, and the relative stronger output power ratio with a value of (1-R). Moreover, in the case of increasing values of $\theta$ (i.e., 15/85 A-MMI), the left-hand side of the weak output's taper $\left(\Delta^{*}\right)$ has to be reduced ([Fig. 2.2) to be consistent with the layout of the device.

\subsection{Measurements and Analysis}

The designed devices have been fabricated on standard silicon-on-insulator (SOI) samples with a top silicon layer thickness of $220 \mathrm{~nm}$ (resistivity $\rho=1-10 \Omega / \mathrm{cm}$ ) and a buried oxide layer thickness of $2 \mu \mathrm{m}$. To obtain the desired $70 \mathrm{~nm}$ depth etch for both waveguides and grating couplers, the fabrication was performed with electron beam (RAITH 150), using a direct writing process performed on a coated $100 \mathrm{~nm}$ hydrogen silsesquioxane resist film. The process was optimized to reach the required 


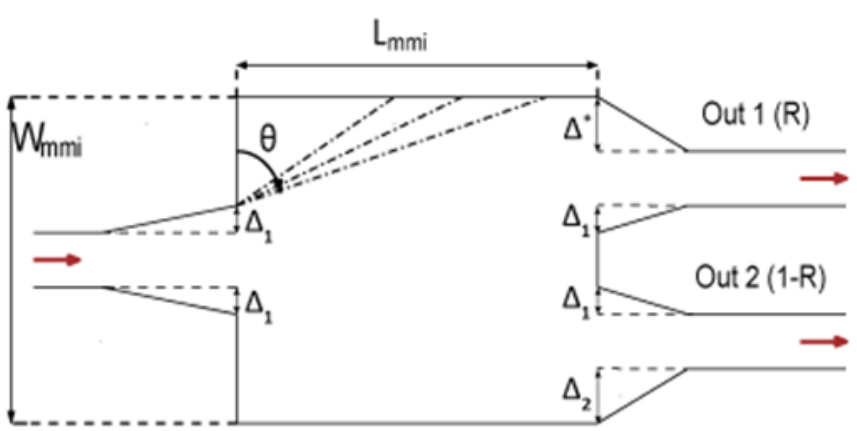

(a)

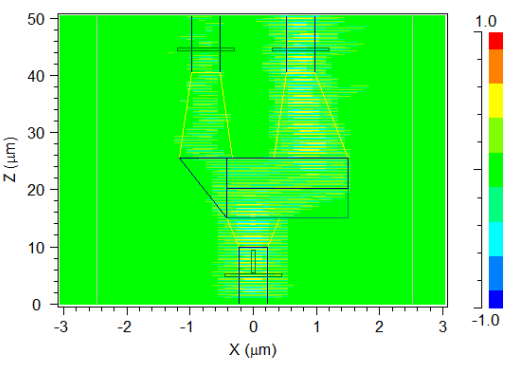

(b)

Figure 2.2: (a) 2D schematic of a 1-by-2 A-MMI power splitter, which shows the design parameters. (b) Distribution of the magnetic field $\left(H_{y}\right)$ in the multimode region of the asymmetrical MMI $(15 / 85$ A-MMI $)\left(\Delta^{*}=0.2 \mu \mathrm{m}\right)$.

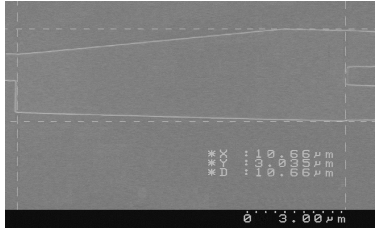

(a)

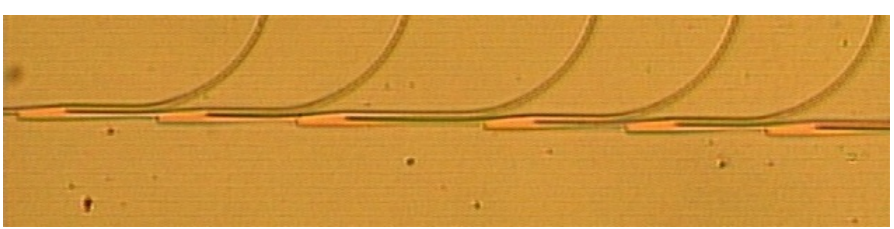

(b)

Figure 2.3: (a) SEM image of the A-MMI splitter with a ratio of 30/70 $\left(\theta \sim 83^{\circ}\right)$. (b) Snapshot of the cascade of A-MMI used for the measurements.

dimensions employing an acceleration voltage of $30 \mathrm{keV}$ and an aperture size of 30 $\mu \mathrm{m}$. After developing the HSQ resist using tetramethylammonium hydroxide as developer, the resist patterns were transferred into the SOI samples employing an also optimized "inductively coupled plasma-reactive ion etching" (ICP-RIE) process with fluoride gases. The shallow-etched passive silicon chip was then covered with $700 \mathrm{~nm}$ thick silica, using plasma-enhanced chemical vapor deposition (PECVD) at $400^{\circ} \mathrm{C}$ (Centura P5200).

We fabricated four different versions of the device, with four distinct values of $\mathrm{R}$, namely, $15 \%, 25 \%, 30 \%$, and 40\%. This allowed us to evaluate the validity of our assumptions and flexibility of the design.

The devices have been measured using the vertical coupling setup depicted in Fig. 2.4. The CW laser input is polarized manually in order to maximize the coupling with the input grating couplers of the device under test (DUT). The output fiber is 
a)

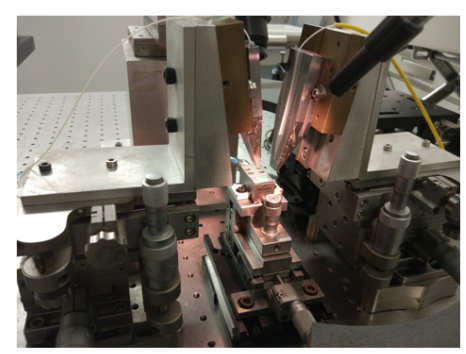

b)

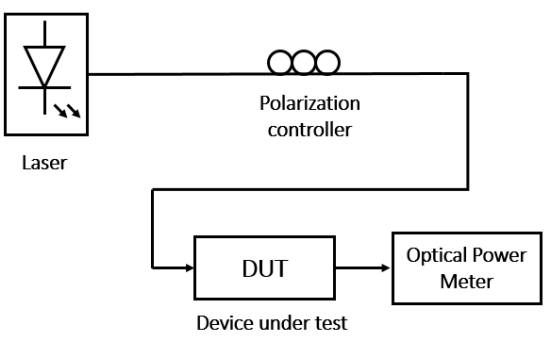

Figure 2.4: (a) Photo of the DUT and (b) schematic of the setup used for the characterization.

connected to a power meter. As far as the characterisation is concerned, the devices are arranged in a cascaded configuration, where each "weak output" is measured and the "strong output" is connected to the subsequent A-MMI. Figure 2.3 shows the cascade arrangement and a SEM image of a single A-MMI. Therefore, using a nonlinear regression process, the output ratios $(\mathrm{R})$ are calculated for each version of the device in the wavelength range 1540-1580 nm, via the use of a tunable laser (SANTEC TSL 210-F). The insertion losses and R calculations are summarized in Figs. 2.5. Figure 2.5(a) shows a small discrepancy, which exhibit a variation of up to $\sim 3 \%$ above the simulation results. This can be ascribed to fluctuations caused by possible minor deviations on the device fabrication and data analysis process. Moreover, Fig. 2.5(c) shows the behaviour at $1.55 \mu \mathrm{m}$, from which we can observe the quasi-asymptotic dependence of the splitting ratio for values of the angle $\theta$ below $75^{\circ}$. These results give some insights on the achievable values of $R$, although power splitting ratio values ranging from $15 / 85$ to 40/60 could readily be obtained. Interestingly, regardless of the fact that the multimode cavity of our asymmetric MMI splitting devices is altered in relation to their 50:50 symmetric counterparts, they keep maintaining the broadband characteristic of the latter (Fig. 2.5(a)). Overall, the results show stable power splitting ratio dependence versus wavelength, despite a slight decrease of $\mathrm{R}$ as the wavelength increases. We believe that this behaviour is caused by the intrinsic nature of multimode interference devices. As reported by Soldano et al. [14], the wavelength causes the interference pattern to change. As a result, the beating length of the device changes and causes the twofold image to move back accordingly. However, the presence of edgeless tapers at the outputs helps compensate and reduce to some extent this slightly decreasing trend.

The overall losses of the A-MMI, as illustrated by Fig. 2.5(b), are in the range 


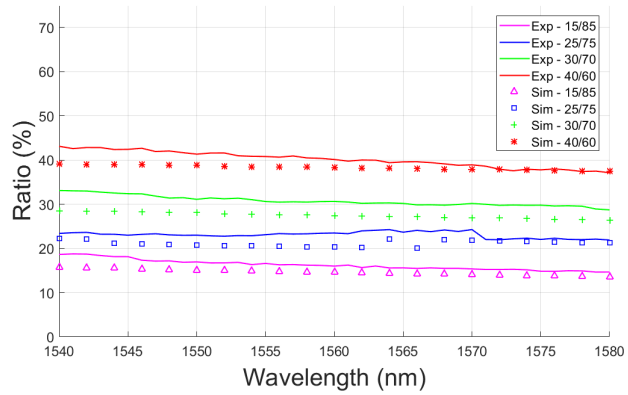

(a)

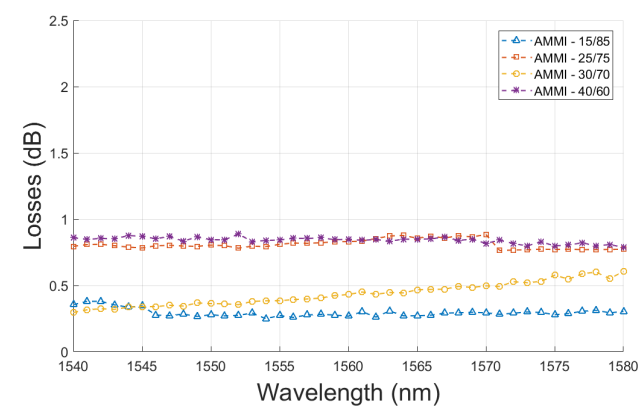

(b)

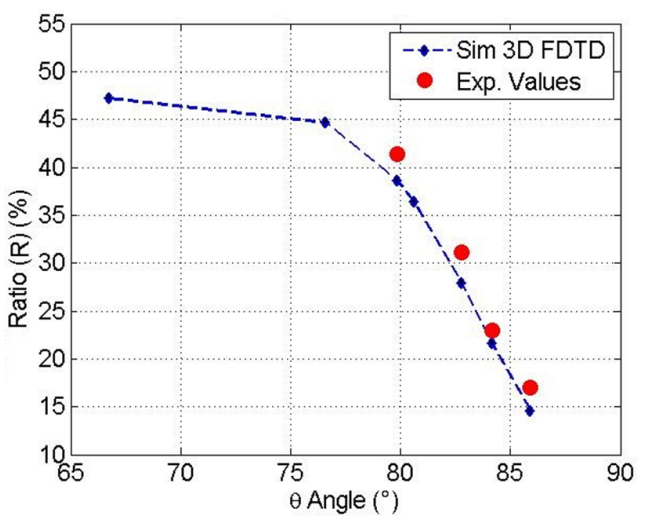

(c)

Figure 2.5: (a) Wavelength dependence of the average splitting ratio (R) in the range 1540$1580 \mathrm{~nm}$, where we can observe the MMI broadband operation. (b) Wavelength dependence of the device losses in the range 1540-1580 $\mathrm{nm}$. The losses are in the range $\sim 0.4-0.8 \mathrm{~dB}$ for the four considered geometries. (c) Graph showing the details of the dependence of the splitting ratio upon $\theta$ at $1550 \mathrm{~nm}$. The experimental values deviate exhibit a deviation up to a $\sim 3 \%$ from the 3D-FDTD simulations results.

$\sim 0.4-0.8 \mathrm{~dB}$; this value is slightly higher of a conventional multimode interference devices [14, 16, 17]. We believe this higher value of losses could be attributed to the higher roughness of the device caused by the breaking of the cavity symmetry or to some minor deviation of the fabrication process, which could cause a systematic error. Further optimization of the fabrication process could reduce these values closer to the theoretical ones of $\sim 0.2-0.3 \mathrm{~dB}$.

In conclusion, a novel A-MMI 1x2 power splitters featuring arbitrary power splitting ratios in a rib configuration have been designed and characterized, in which the asymmetry is determined by altering the device geometry. As a result, it has 
been possible to achieve varying power splitting ratios ranging from 15/85 to 40/60 with relatively low-loss penalty and broadband operation. The dimensions of the proposed device are small $(10.5 \mu \mathrm{m} \times 3 \mu \mathrm{m})$ to minimize the impact on the overall footprint of large scale integrated silicon photonic chips (Si-PICs).

\subsection{Modulator with A-MMI}

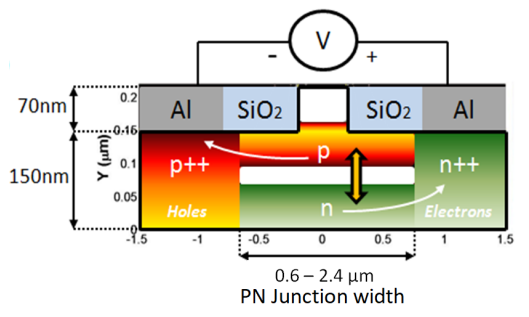

(a)

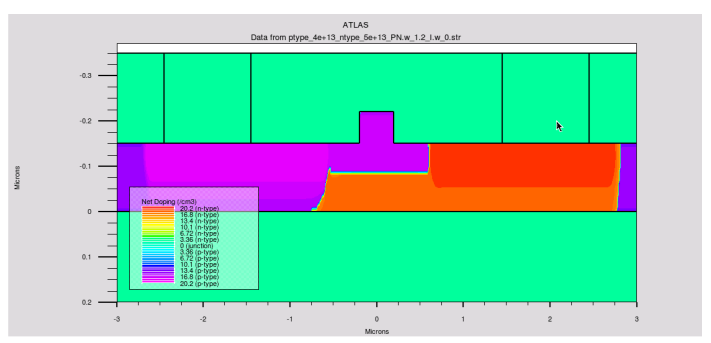

(c)

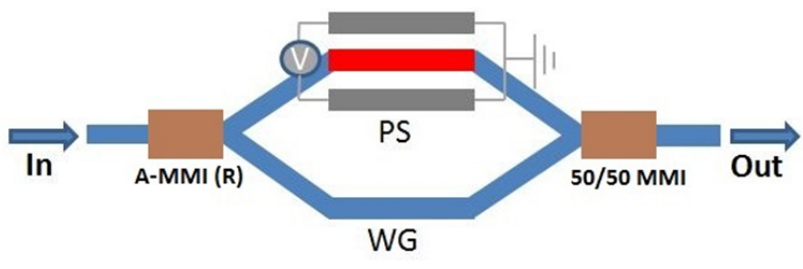

(b)

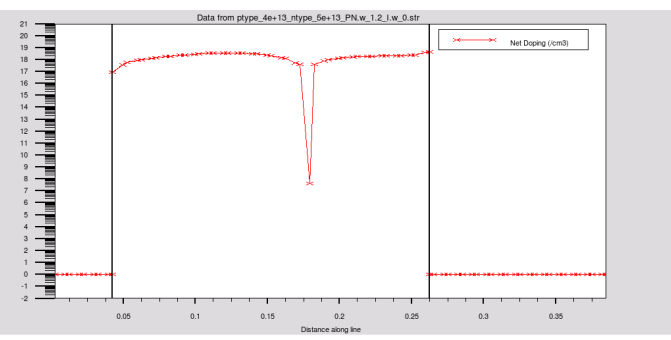

(d)

Figure 2.6: (a) 2D geometry of the vertical pn junction in rib configuration. (b) Schematic of the MZI with single phase shifter and arbitrary splitting ratio. (c) 2D cross section of the simulated pn junction (Silvaco). (d) Net doping profile of the phase shifter.

Here, we present the design of a highly efficient silicon carrier depletion-based modulator in the O-band, which extinction ratio (ER) can be significantly improved via the implementation of asymmetrical multi-mode interference couplers at the expenses of a slight increase in the propagation losses of the device.

The modulator has been designed using a shallow-etched waveguide described previously (70nm etch, 150nm slab) associated with a vertical pn junction. This layout is potentially more tolerant to mask alignment errors since its formation is dictated by the implantation energy of the dopants rather than the resolution of the optical 
lithography. Moreover, the combination of slightly lower optical mode confinement, induced by the rib configuration, and tunable width of the pn junction leads to a higher interaction of the propagating light and charge carriers.

The proposed modulator layout and doping profile of the phase shifter are summarized in Fig. 2.6. It features a single drive symmetric Mach-Zehnder interferometer (MZI) configuration with a single phase shifter and arbitrary power splitting ratios at the input. The implementation of A-MMI allows more power to be injected into the doped arm of the MZI and hence helps compensate for the loss imbalance produced by the active phase shifter. As an increasing amount of power is fed into the active arm, the ER increases and, due to the trade-off imposed by the layout itself, a penalty in terms of losses has to be taken into account, as depicted by Fig. 2.7(a). The phase shifter has been modeled using ATHENA and ATLAS, respectively pro-

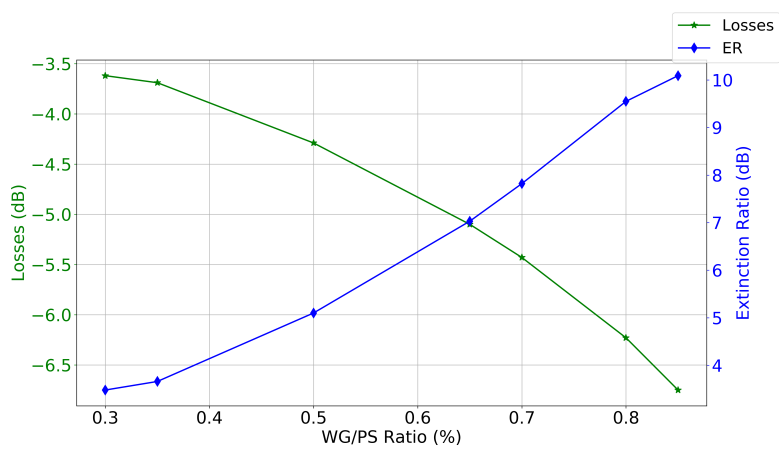

(a)

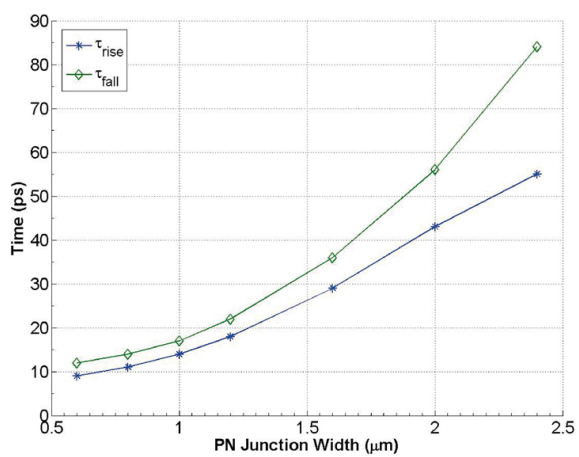

(b)

Figure 2.7: (a) Splitting ratio dependence of insertion losses and extinction ratio. (b) Relation between rise/fall time and width of the pn junction.

cess and device simulation software from SILVACO. It is important to remind how the response time of the phase shifter is affected as the width of pn junction changes. Fig. 2.7(b) shows how progressively reducing the width of pn junction from $2.4 \mu \mathrm{m}$ down to $0.6 \mu \mathrm{m}$ and maintaining the doping doses constant, the rise and fall times can be significantly decreased, allowing us to reach theoretical values of $\sim 10-12$ ps. For this specific layout, a width of $1.2 \mu \mathrm{m}$ for the pn junction and doses of 4e13/5e13 $\mathrm{cm}^{2}$, with implantation energies of $100 / 150 \mathrm{keV}$, for the $\mathrm{p}$ and $\mathrm{n}$ regions respectively, have been chosen. These specifications allow us to obtain good overall performance both in terms of losses, $\sim 4.5 \mathrm{~dB} / \mathrm{mm}$, and a theoretical bandwidth of around 18 
$\mathrm{GHz}$, which should be sufficient to accommodate a the target bit-rate of $25 \mathrm{Gbit} / \mathrm{s}$. In order to evaluate the effects of the implemented A-MMI, the ER and loss penalty at the modulator output have been analytically calculated and Fig. 2.7(a) displays the results. As expected, an increasing amount of power directed to the phase shifter determines an higher ER. However, this situation limit the performances in terms of losses, due to the trade-off required by the proposed layout.

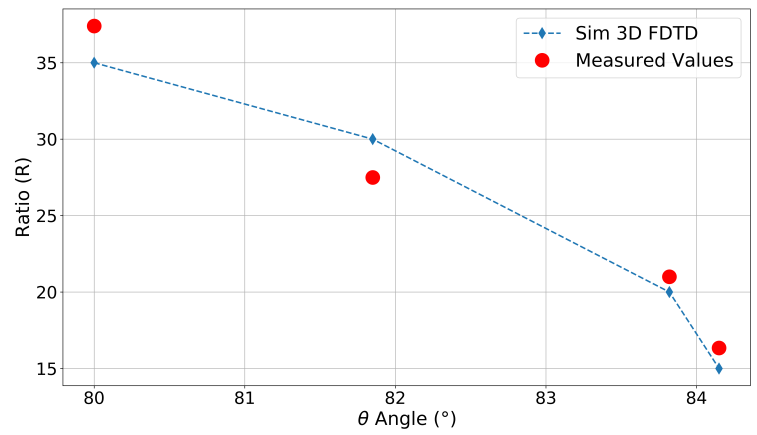

Figure 2.8: Graph shows a detail of the dependence of the splitting ratio from $\theta$ at $1.31 \mu \mathrm{m}$.

The A-MMI has been redesigned for broadband operation at $1.31 \mu \mathrm{m}$, using the same approach described previously in Sec. 2.1. The resulting dimensions are: $L_{M M I}=8.6$ $\mu \mathrm{m}$ and $W_{M M I}=2.5 \mu \mathrm{m}$. Four different versions of the A-MMI have been designed with splitting ratios of 15/85, 20/80, 30/70 and 35/65. The fabricated A-MMIs show similar broadband performance to the versions in the C-band. The insertion losses have been slightly reduced $(\sim 0.3 \mathrm{~dB})$ thanks to improvements in the fabrication process, and the resulting splitting ratios are within reasonable variance values, 3-4 \% of the designed ones, as shown in Fig. 2.8 .

\subsection{Modulator with A-MMI results}

The design A-MMIs have been implemented in six different layouts of MZM. The modulator itself featured a symmetric MZI configuration with a phase-shifter length of $1 \mathrm{~mm}$, and with proper RF travelling wave electrodes . An image of the overall design can be observed in Fig. 2.9. (a). 
The devices have been characterized using a vertical coupling setup and the generic setup is summarized in Fig. 2.9(b). The data transmission measurements have been realised driving the modulator with a non-return to zero (NRZ) pseudo-random bit sequence $\left(\mathrm{PRBS}^{-1}\right.$ ) generated by a bit pattern generator (SHF BPG 44E). The electrical signal has been amplified through a high-speed RF amplifier (SSHF-810) to achieve the target voltage swing $\left(V_{p p}\right)$ and then combined to a DC bias using a bias-tee. The modulating signal is applied to the travelling wave electrodes, which are terminated by a $50 \Omega$ external resistance. Finally, the output optical signal is detected via a $40 \mathrm{GHz}$ digital communication analyser (Infiniium DCA-J 86100C).

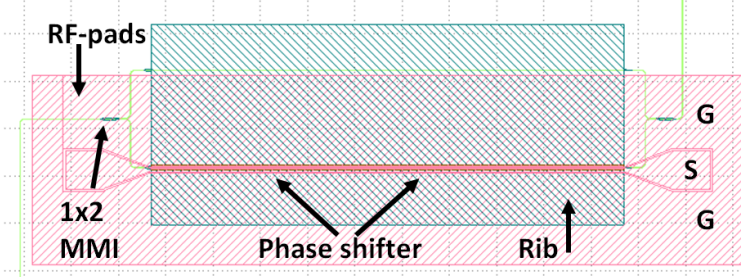

(a)

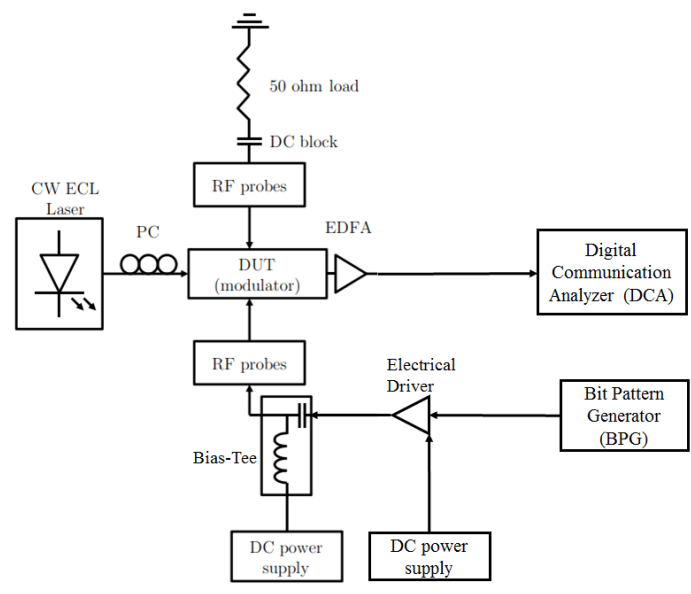

(b)

Figure 2.9: (a) Layout overview of the device. (b) Schematic of the characterisation setup for the MZM.

Firstly, the devices have been characterized passively, in order to evaluate losses of the phase shifter and to observe their consistency in relation to the SILVACO simulations. Then, DC and RF measurements have been carried out allowing to check the capability of these modulators to deliver the expected bit-rates. Finally, 
the measured ER and output losses have been compared to the analytical results presented in Fig. 2.7(a).

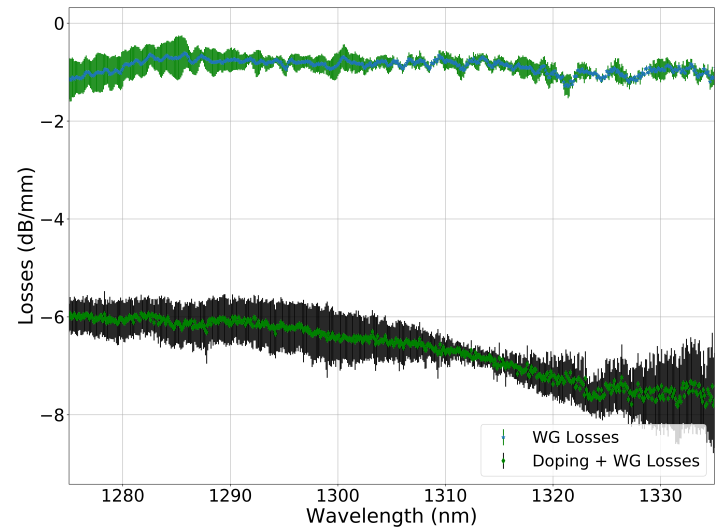

(a)

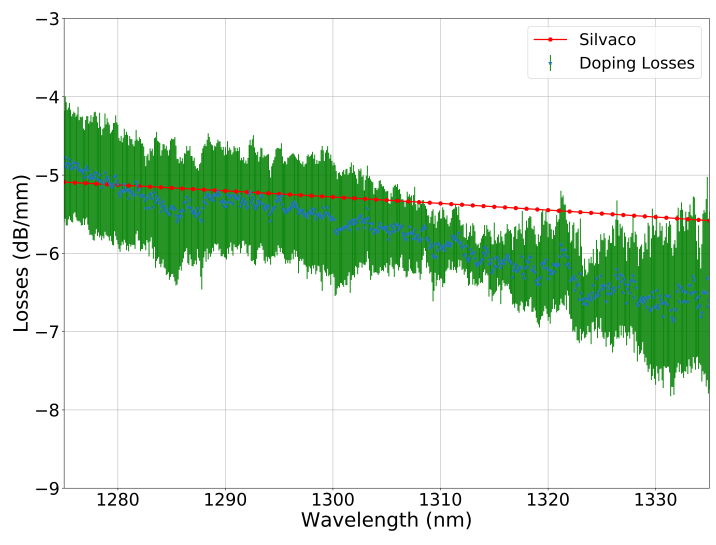

(b)

Figure 2.10: (a) Graph shows the propagation losses of the doped phase shifter $(\sim 6-7 \mathrm{~dB} / \mathrm{mm})$ compared to that the of a reference standard rib waveguide $(\sim 1 \mathrm{~dB} / \mathrm{mm})$ of the same chip. It shows the high contribution of the doping on the overall power budget of the modulator. (b) Propagation losses caused by the doping inside the phase shifter only are reported. The measured values show good agreement with the simulation.

Figure 2.10 summarise the phase shifter performance. The losses contributions arises from are the waveguide's sidewall roughness and the dopants. In Fig. 2.10(a) one can observe how the waveguide contribution, caused mainly by sidewall roughness [18, 19, 20] is relatively small $(\sim 1 \mathrm{~dB} / \mathrm{mm})$ when compared to the overall losses of a phase shifter, which are much higher $(\sim 6-7 \mathrm{~dB} / \mathrm{mm})$. As a result, the losses associated to only doping are $\sim 4-5 \mathrm{~dB} / \mathrm{mm}$, which is in good agreement compared to the simulated results (Fig. 2.10).

Following the passive characterisation, the DUT have been characterized in DC. As a reference, the resulting spectra of the $65 / 35$ version of the electro-optical modulator are summarized in Fig. 2.11(a). It is important to notice that the device has been characterised only in reverse bias and threshold regime (i.e. just below the pn junction threshold regime), due to the fact that the phase shifter was designed and optimised for high-speed operations, which can be only achieved when working in these regimes [21, 22]. 


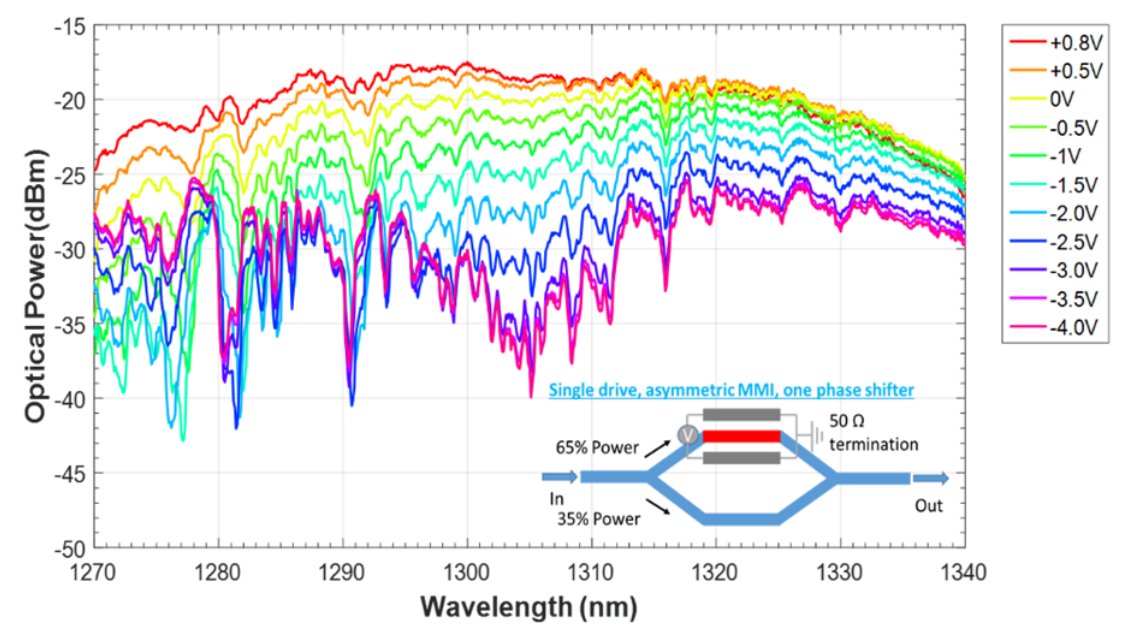

(a)

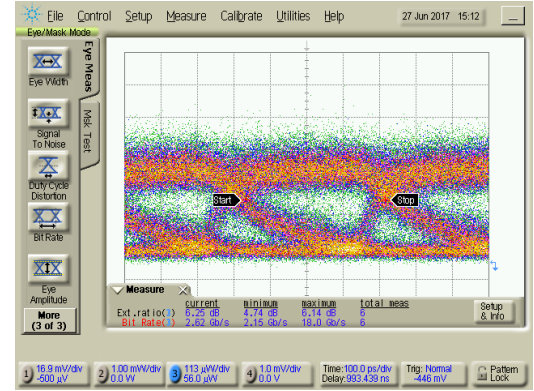

(b)

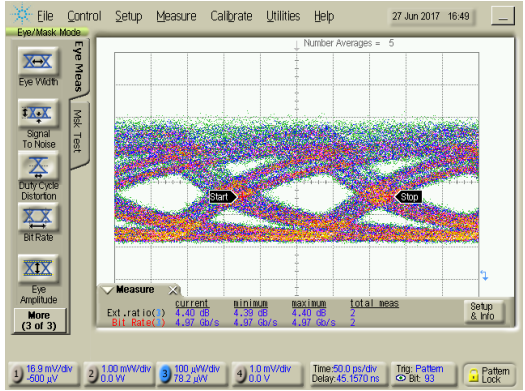

(c)

Figure 2.11: (a) DC response of a 65/35 MZM. (b) Eye-diagram of the MZM @ 4 Gbit/s (ER $=6.25 \mathrm{~dB}) .(\mathrm{c})$ Eye-diagram of the MZM @ 5 Gbit $/ \mathrm{s}(\mathrm{ER}=4.40 \mathrm{~dB})$ operating at $1305 \mathrm{~nm}$.

The image shows how the optical power varies for varying applied DC voltages, giving as a result an efficiency of $V_{\pi} L_{\pi} \simeq 0.35-0.40 \mathrm{~V}$.cm, which is comparable to other similar devices [23]. More specifically, due to the design of the phase shifter, the output optical power decreases when the reverse bias increases, and on the other hand increases slightly when going into threshold regime.

Fig. 2.11(b)-(c) depicts the RF performances of the modulator with $V_{D C}=1.0 \mathrm{~V}$ and $V_{p p}=2.55 \mathrm{~V}$. It was possible to only measure speeds of up to $4 \mathrm{Gbit} / \mathrm{s}(\mathrm{ER}=$ $6.25 \mathrm{~dB})$ and $5 \mathrm{Gbit} / \mathrm{s}(\mathrm{ER}=4.40 \mathrm{~dB})$. The main cause for these limited speeds is the metal stack that delivers the electrical signal to pn junction, as discussed previously in Sec. 1.4. Therefore, it is mandatory to solve this issue in the next generation of modulators, designing optimized travelling wave electrodes that take 
into account the constraints of the metal stack.

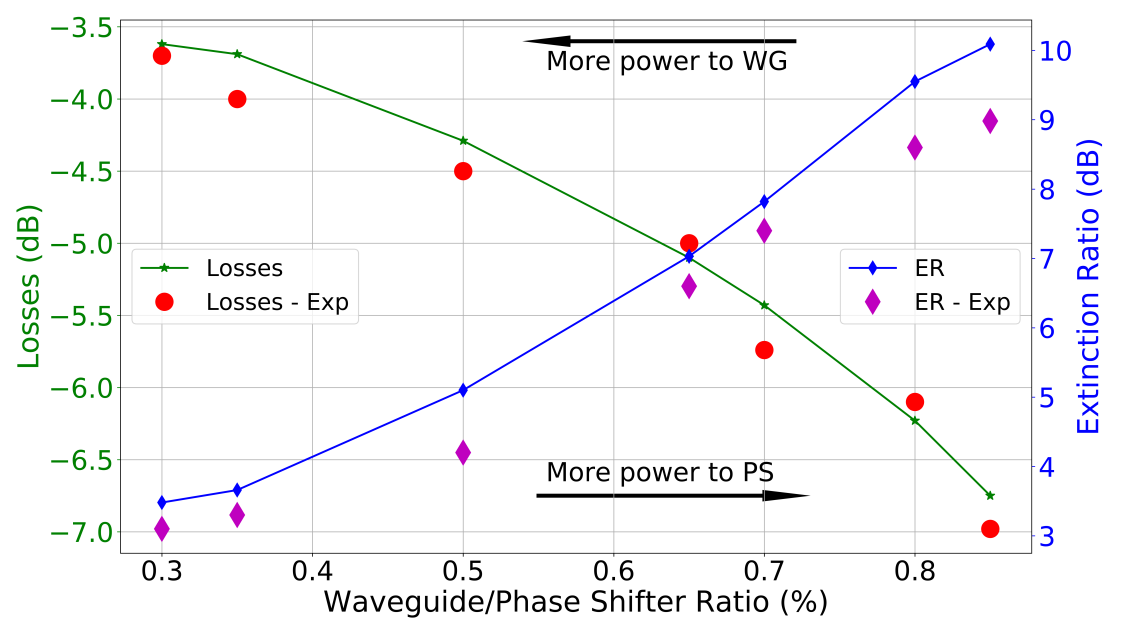

Figure 2.12: Comparison of the analytical and measured results of the modulators, in terms of insertion losses and extinction ratio versus reference waveguide (WG) - phase shifter (PS) power ratio.

The last aspect to analyse is how the MZM's ER and losses compare to the design values (Fig. 2.12). Overall, the analytical and experimental results are in good agreement, especially in terms of optical losses at the modulator output. On the other hand, the measured ER values are all below what is predicted by the analytical model. We retain this discrepancy to be caused by small inconsistencies in the phase shifter's doping profiles induced by small fluctuations in the fabrication process.

\subsection{Conclusions}

In conclusion, we have demonstrated the design, fabrication and measurements of a novel asymmetrical multimode interference (A-MMI) device for SOI platforms with tunable splitting ratios. Its small footprint, high bandwidth and low-losses makes it an ideal candidate for applications in photonic integrated circuits. Key aspects such as losses as well as sidewall roughness must be improved for future generation of devices.

Furthermore, the device has been implemented in a MZM with good results. This 
allowed to increase the tunability of the device allowing for precise control over the ER-losses ratio without any loss in terms of performance. This increases its attractiveness for more complex applications in PIC, due to its robustness and high fabrication tolerance when compared to other passive devices such as direction coupler. 


\section{Bibliography}

[1] Christopher Batten, Ajay Joshi, Jason Orcutt, Anatol Khilo, Benjamin Moss, Charles W Holzwarth, Miloš A Popovic, Hanqing Li, Henry I Smith, Judy L Hoyt, et al. Building many-core processor-to-dram networks with monolithic cmos silicon photonics. IEEE Micro, 29(4):8-21, 2009.

[2] Richard Soref. The past, present, and future of silicon photonics. IEEE Journal of selected topics in quantum electronics, 12(6):1678-1687, 2006.

[3] David J Lockwood and Lorenzo Pavesi. Silicon photonics. Berlin: Springer, 2004.

[4] Tao Liu, Armis R Zakharian, Mahmoud Fallahi, Jerome V Moloney, and Masud Mansuripur. Multimode interference-based photonic crystal waveguide power splitter. Journal of lightwave technology, 22(12):2842, 2004.

[5] Hong Yang, Guo-Qing Qin, Hao Zhang, Xuan Mao, Min Wang, and Gui-Lu Long. Multimode interference induced optical nonreciprocity and routing in an optical microcavity. arXiv preprint arXiv:2001.10713, 2020.

[6] Zhanghua Han and Sailing He. Multimode interference effect in plasmonic subwavelength waveguides and an ultra-compact power splitter. Optics communications, 278(1):199-203, 2007.

[7] Andrea Zanzi, Christos Vagionas, Amadeu Griol, Alvaro Rosa, Sergio Lechago, Miltiadis Moralis-Pegios, Konstantinos Vyrsokinos, Nikos Pleros, Jochen Kraft, Victor Sidorov, et al. Alignment tolerant, low voltage, 0.23 v. cm, push-pull silicon photonic switches based on a vertical pn junction. Optics express, 27(22):32409-32426, 2019.

[8] A Ortega-Monux, L Zavargo-Peche, A Maese-Novo, I Molina-Fernández, R Halir, JG Wanguemert-Perez, P Cheben, and JH Schmid. High-performance multimode interference coupler in silicon waveguides with subwavelength structures. IEEE Photonics Technology Letters, 23(19):1406-1408, 2011.

[9] Chao Li, Tigang Ning, Xiaodong Wen, Jing Li, Jingjing Zheng, Haidong You, Hongyao Chen, Chan Zhang, and Wei Jian. Strain and temperature discrim- 
ination using a fiber bragg grating and multimode interference effects. Optics Communications, 343:6-9, 2015.

[10] Robert Halir, Günther Roelkens, A Ortega-Moñux, and JG Wangüemert-Pérez. High-performance 90 hybrid based on a silicon-on-insulator multimode interference coupler. Optics letters, 36(2):178-180, 2011.

[11] Kimmo Solehmainen, Markku Kapulainen, Mikko Harjanne, and Timo Aalto. Adiabatic and multimode interference couplers on silicon-on-insulator. IEEE Photonics Technology Letters, 18(21):2287-2289, 2006.

[12] Andrea Zanzi, Antoine Brimont, Amadeu Griol, Pablo Sanchis, and Javier Marti. Compact and low-loss asymmetrical multimode interference splitter for power monitoring applications. Optics letters, 41(2):227-229, 2016.

[13] Maurus Bachmann, Pierre A Besse, and Hans Melchior. General self-imaging properties in $\mathrm{n} \times \mathrm{n}$ multimode interference couplers including phase relations. Applied optics, 33(18):3905-3911, 1994.

[14] Lucas B Soldano and Erik CM Pennings. Optical multi-mode interference devices based on self-imaging: principles and applications. Journal of lightwave technology, 13(4):615-627, 1995.

[15] Pierre A Besse, Maurus Bachmann, Hans Melchior, Lucas B Soldano, and Meint K Smit. Optical bandwidth and fabrication tolerances of multimode interference couplers. Journal of Lightwave Technology, 12(6):1004-1009, 1994.

[16] Hui Chen and Andrew W Poon. Low-loss multimode-interference-based crossings for silicon wire waveguides. IEEE photonics technology letters, 18(21):2260-2262, 2006.

[17] Hongzhen Wei, Jinzhong Yu, Xiaofeng Zhang, and Zhongli Liu. Compact 3-db tapered multimode interference coupler in silicon-on-insulator. Optics letters, 26(12):878-880, 2001.

[18] Tymon Barwicz and Hermann A Haus. Three-dimensional analysis of scattering losses due to sidewall roughness in microphotonic waveguides. Journal of Lightwave Technology, 23(9):2719, 2005. 
[19] F Grillot, L Vivien, S Laval, D Pascal, and E Cassan. Size influence on the propagation loss induced by sidewall roughness in ultrasmall soi waveguides. IEEE Photonics Technology Letters, 16(7):1661-1663, 2004.

[20] Kevin K Lee, Desmond R Lim, Hsin-Chiao Luan, Anuradha Agarwal, James Foresi, and Lionel C Kimerling. Effect of size and roughness on light transmission in a si/sio 2 waveguide: Experiments and model. Applied Physics Letters, 77(11):1617-1619, 2000.

[21] Carlos Angulo Barrios, Vilson R de Almeida, and Michal Lipson. Low-powerconsumption short-length and high-modulation-depth silicon electrooptic modulator. Journal of Lightwave Technology, 21(4):1089, 2003.

[22] Randolph E Bank, William M Coughran, Wolfgang Fichtner, Eric H Grosse, Donald J Rose, and R Kent Smith. Transient simulation of silicon devices and circuits. IEEE Transactions on Computer-Aided Design of Integrated Circuits and Systems, 4(4):436-451, 1985.

[23] A Brimont, DJ Thomson, P Sanchis, J Herrera, FY Gardes, JM Fedeli, GT Reed, and J Martí. High speed silicon electro-optical modulators enhanced via slow light propagation. Optics Express, 19(21):20876-20885, 2011. 



\section{Chapter 3}

\section{D Photonic crystal design}

This chapter is devoted to the design and characterization of two novel slow wave waveguides, which exhibit relatively high group indices and high Fabry-Pèrot resonance suppression.

\subsection{Background}

In the last decades, research has been performed to realize high-level integration of silicon photonics for optical interconnects and networks [1, 2], optical computing [3, 4], and biosensing [5]. This is primarily driven by the fact that silicon-on-insulator (SOI) technology is fully compatible and scalable with complementary metal oxide semiconductor (CMOS) technology and it has the characteristic to be a high refractive index platform, which enables such compact and efficient devices. Recent efforts in silicon photonic devices, such as III-V/silicon hybrid lasers [6], modulators [7, 8], and switches [9, 10, 11], have all paved a path toward realizing silicon-based high-density electronic and photonic integration circuits (EPICs) [12]. However, due to high-index contrast, photonic devices based on SOI waveguides always suffer from different issues, including high polarization sensitivity, limited bandwidth, an high level of phase errors caused by fabrication, thermal sensitivity, and relatively large propagation loss [13]. Those issues greatly limit application range of silicon-based photonic devices. There has been considerable effort by the international community to improve device performances by designing new structures, and photonic crystals are the most promising option. 
Photonic crystal (PhC) structures were firstly introduced by Yablonovich [14] and John [15]. The idea covers the concepts behind the $\mathrm{PhC}$ design material structures. The PhCs material structures affect the properties of the photons in the similar behaviour to semiconductor affect the properties of electrons. The concept suggestes that structures with periodic variations in dielectric constant might affect the photonic modes in a material. In a semiconductor, the atomic lattice provides a periodic potential for the electrons to propagate through the electronics crystal. The potential creates a gap in the energy to forbid the electrons to propagate in any direction. In $\mathrm{PhCs}$, the lattice periodicity variation in refractive index forms a photonic bandgap (PBG) in the crystal. For a full bandgap light is forbidden to propagate in any direction similar to carriers in a semiconductor. In PBG, the light propagation is prohibited and completely reflected inside the PhCs structure. The PBG is an important discovery that has led to various scientific engineering applications, i.e. the control of spontaneous emission [16], trapping of photons [17], and several other applications $[\mathrm{REF}]$. PhCs can be generally divided into one-dimensional (1D), twodimensional (2D) and three-dimension (3D) arrangements. In 1D PhCs, the light travels in periodic modulation of permittivity, which occurs only in one direction (i.e. $\mathrm{x}$-direction). An example of $1 \mathrm{D}-\mathrm{PhC}$ is Bragg gratings that are widely used in vertical cavity surface emitting lasers (VCSELs) [18]. In 2D-PhCs, the periodicity of the permittivity is along two directions with the third direction of the medium is uniform (i.e. $\mathrm{x}$ - and ydirection). In 3D-PhCs, the permittivity modulation is along all three directions (i.e. $\mathrm{x}-, \mathrm{y}$ - and z-directions). Examples for 1D, 2D and 3D PhCs are given in [19]. Design and creation of 3D-PhC $([20,21,22])$ have been realized as predicted by [14]. However, still to this day, such structures are still very challenging to fabricate, and therefore their realization is still limited.

Over the recent past, photonic crystal development allowed the creation of widespread application of photonic integrated circuits (PIC), i.e. low-loss waveguide crossings [23], ultra-broadband multimode interference (MMI) couplers [24] and fiber-chip grating couplers [25]. 


\subsection{Slow light structure design}

The design of the 1-dimensional photonic crystal (1D-PhC) have been carried out using several tools, such as Plane Wave Expansion (PWE) as a first step, then 3D and 2D Finite-Difference Time-Domain (FDTD) simulations as a second step, for the apodized versions.

PWE allows for a rapid study of the band structure of the periodic building block of the $1 \mathrm{D} \mathrm{PhC}$ to set up the desired parameters of the final design. Moreover, both 3D and 2D FDTD simulations have been used to fine tune and characterise the apodizated structure. However, the exclusive use of FDTD is required when dealing with non-uniform 1D PhC, due to the inability of PWE to properly simulate such structures. Besides, the use of 3D simulations has been reduced when possible, due to the high memory and computational power required by the FDTD algorithm. As a result, 2D FDTD with vEIM (variational Effective Index Method [26]) simulations have been used to simulate longer structures, providing fairly accurate results when compared to 3D FDTD simulations. The number of parameters of this particular structure are fairly high, however they can be reduced firstly by the constraints of the Silicon-on-insulator (SOI) platform with which we are working with. The height of the silicon layer is fixed to $220 \mathrm{~nm}$, and the etch depth has to be of $70 \mathrm{~nm}$ (Fig. 3.1(a)). Therefore, the remaining parameters $\left(W_{i}, W_{e}, L_{i}, L_{e}, \Lambda\right)$ have to be determined, as depicted in Fig. 3.1(b).

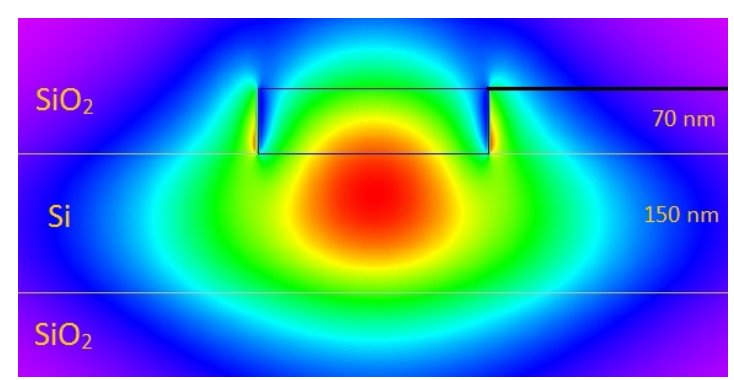

(a)

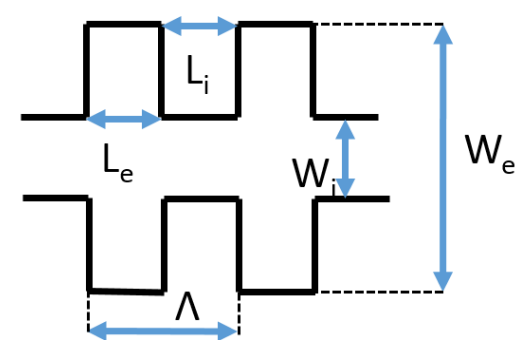

(b)

Figure 3.1: (a) Vertical cross-section of the waveguide. (b) Schematic and geometry of the 1D $\mathrm{PhC}$.

As the input waveguide of the structure have been set to $400 \mathrm{~nm}$, to ensure single 
mode operation at $1.31 \mu \mathrm{m}$, the width of the inner part of the $1 \mathrm{D} \mathrm{PhC}\left(W_{i}\right)$ have been set to $400 \mathrm{~nm}$ as well in order to minimise possible coupling losses due to the backwards reflections induced by the optical mismatch. Similarly, We has been chosen to be $1.0 \mu \mathrm{m}$. This choice has been dictated by the geometry of the pn junction previously presented, in order to avoid any potential overlap between the slow light mode and the heavily doped region, which would increase significantly the losses due to the higher doping concentration.

The subsequent main parameter to be determined is the period $(\Lambda)$, which has to be properly chosen to allow $1 \mathrm{D} \mathrm{PhC}$ to operate in the wavelength range of interest. It can be approximately determined starting from the Bragg condition:

$$
\Lambda n_{\text {eff }}=m \frac{\lambda_{\text {Bragg }}}{2}
$$

where $n_{\text {eff }}$ is the effective refractive index of the optical mode, $m$ is the mode order and $\lambda_{\text {Bragg }}$ is the Bragg wavelength.

However, for our specific case, the formula has to be modified, according to [27].

Starting from:

$$
L_{i} n_{\text {eff }}^{W i}+L_{e} n_{e f f}^{W e}=m \frac{\lambda_{B r a g g}}{2}
$$

the considering a filling factor of 0.5 and rearranging for $\Lambda$, we obtain:

$$
\Lambda=m \frac{\lambda_{\text {Bragg }}}{n_{\text {eff }}^{W i}+n_{\text {eff }}^{W e}}
$$

Eq. 3.3 shows that to properly approximate $\Lambda$, the effective refractive index of the wide and narrow sections of the $\mathrm{PhC}$ must be calculated. In order to obtain these values, a waveguide mode solver [28] have been used and the resulting values are: $n_{\text {eff }}^{W i}=2.8248$ and $n_{\text {eff }}^{W e}=2.9398$, which result in $\Lambda \simeq 227 \mathrm{~nm}$ (Fig. 3.2. These parameters allow us to simulate and adjust the structure to our requirements using PWE1 After careful simulations, the final parameters for the design are: $\Lambda=230 \mathrm{~nm}$, $L_{i}=120 \mathrm{~nm}$ and $L_{e}=110 \mathrm{~nm}$. The small changes have been required mainly in order to ensure that there are three different bands in the wavelength range of interest (1270-1350 nm), but also to be able to simulate it efficiently. The resulting band structure with its related optical modes is reported in Fig. 3.3. Close to the band edges, two TE-like modes, for the $1^{\text {st }}$ and $2^{\text {nd }}$ band $(\mathrm{m}=0$ and $\mathrm{m}=1)$, and a TM-like

\footnotetext{
${ }^{1}$ Modeled with BandSOLVE ${ }^{T M}-\mathrm{RSoft}^{T M}$ by Synopsys ${ }^{T M}$ [29]
} 


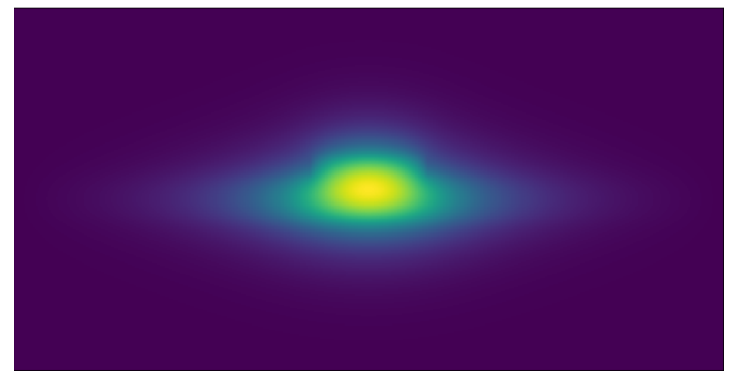

(a)

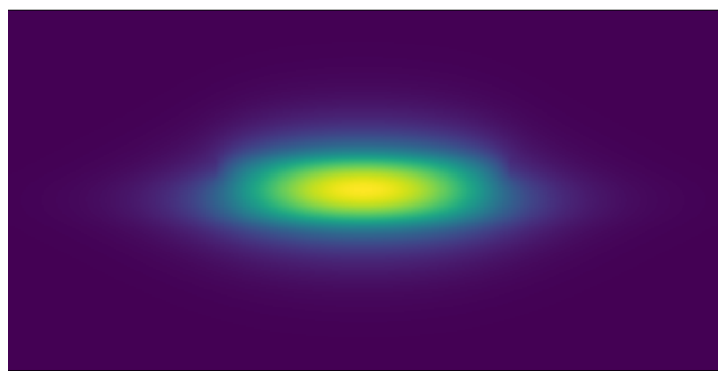

(b)

Figure 3.2: (a) Cross-sections of the optical mode for the (a) $400 \mathrm{~nm}$ and (b) $1 \mu \mathrm{m}$ section of the PhC. Effective refractive index are respectively $n_{e f f}^{W i}=2.8248$ and $n_{\text {eff }}^{W e}=2.9398$.

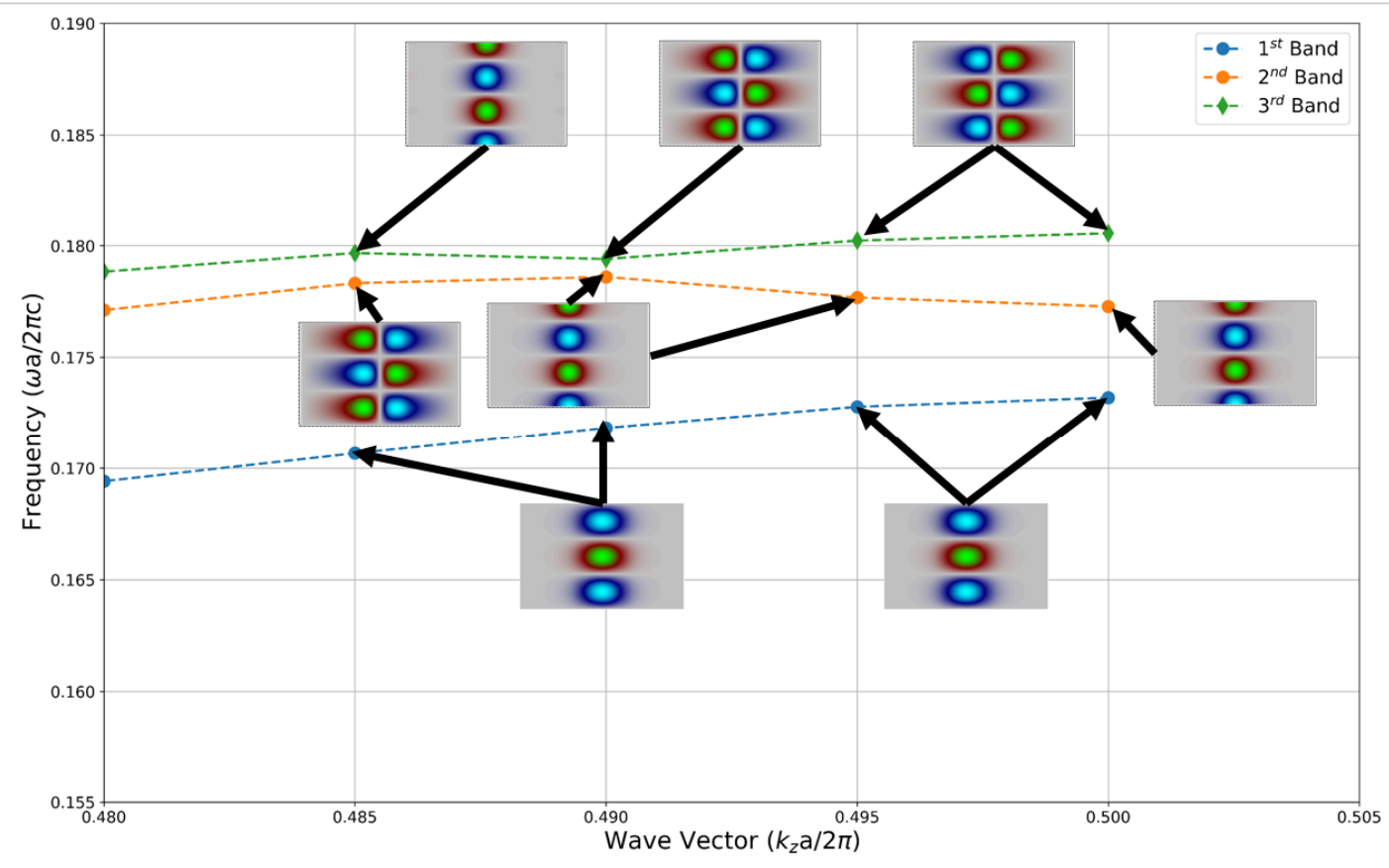

Figure 3.3: Band structure of the designed 1D-PhC and related optical modes.

mode can be identified. The TE-like modes are located respectively in the centre of the wide and narrow part of the PhC. Conversely, the TM-like mode is located in the sidewall gratings of the structure. Interestingly, we can observe that the second and third bands are two hybrid bands. An anti-crossing phenomenon occurs, which is caused by the periodicity added to the silicon waveguide. This opens up the PBG and also causes the bands to couple at their intersection point, causing them to split and form a hybrid band that transitions from one field pattern to the other [30]. 
a)

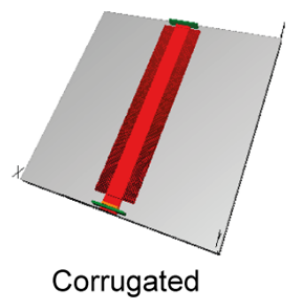

C)

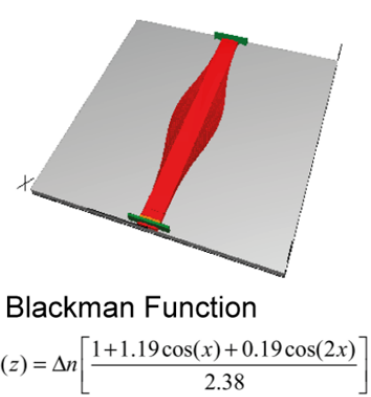

b)

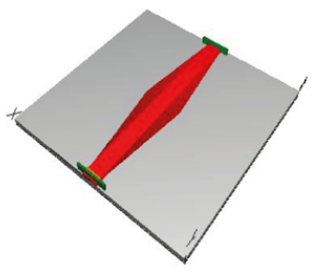

Linear Function

d)

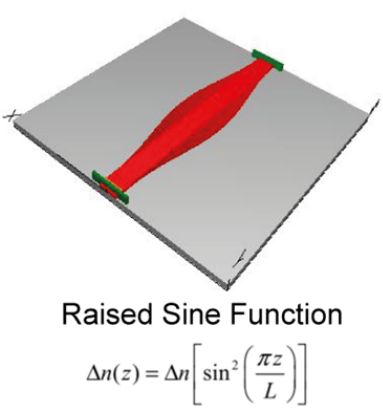

Figure 3.4: Sketch of the 1D tapers under investigation: (a) Standard 1D PhC; (b) Linear taper; (c) Blackman taper and (d) Raised Sine taper.

As said previously, PWE is unable to deal with apodized structure, therefore it is mandatory to use FDTD.

Most of the presented simulations have been performed using 2D FDTD associated with vEIM [26], using a commercial software ${ }^{2}$ and an in-house code (Appendix C), and after having tested the consistency of the 3D-to-2D approximation. Three different configurations have been investigated, namely Blackman, Raised Sine and Linear (Fig. 3.4). The simulated spectra are summarized in Fig. 3.5.

As can be observed, the untapered 1D-PhC has really strong Fabry-Perot resonances, which decrease significantly the efficiency and overall performances of the any potential devices. The solution that we propose is the implementation of an apodisation function, that acts over the sidewall gratings $\left(W_{e}\right)$ of the $1 \mathrm{D}-\mathrm{PhC}$. Indeed, the simulated apodized structures enables the sidelobes to be efficiently minimized while maintaining the position of the PBG as well as the extinction ratio. Therefore, the use of the apodizing function look promising in order to enhance the performances of this type of $1 \mathrm{D} \mathrm{PhC}$ structure.

\footnotetext{
${ }^{2}$ FullWAVE ${ }^{T M}-$ RSoft $^{T M}$ by Synopsys ${ }^{T M}[\mathbf{R E F}]$
} 


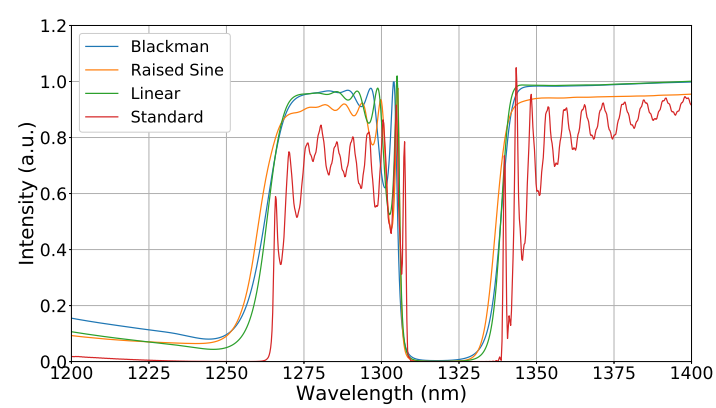

(a)

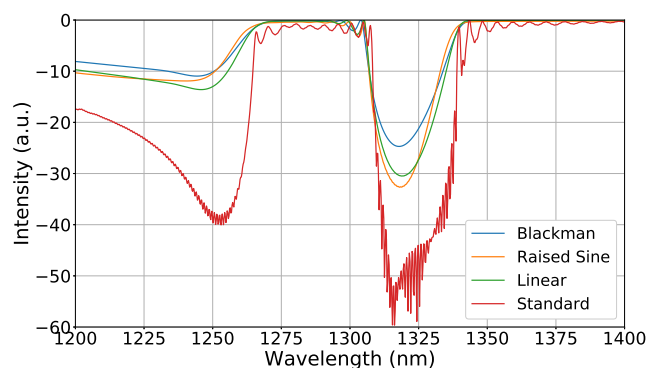

(b)

Figure 3.5: 2D FDTD of the designed structures $(\mathrm{N}=200)$ with vEIM implementation both in (a) linear and (b) logarithmic scales.

\subsection{Single Apodization}

In this section, we present the design and analysis of a $1 \mathrm{D}-\mathrm{PhC}$ with a single apodization. These tapering function have been applied to the wider part of the geometry. The designed devices have been fabricated on standard silicon-on-insulator (SOI) samples with a top silicon layer thickness of $220 \mathrm{~nm}$ (resistivity $\rho=1-10 \Omega / \mathrm{cm}$ ) and a buried oxide layer thickness of $2 \mu \mathrm{m}$. To obtain the desired $70 \mathrm{~nm}$ depth etch for both waveguides and grating couplers, the fabrication was performed with electron beam (RAITH 150), using a direct writing process performed on a coated $100 \mathrm{~nm}$ hydrogen silsesquioxane resist film. The process was optimized to reach the required dimensions employing an acceleration voltage of $30 \mathrm{keV}$ and an aperture size of 30 $\mu \mathrm{m}$. After developing the HSQ resist using tetramethylammonium hydroxide as developer, the resist patterns were transferred into the SOI samples employing an also optimized "inductively coupled plasma-reactive ion etching" (ICP-RIE) process with fluoride gases. The shallow-etched passive silicon chip was then covered with $700 \mathrm{~nm}$ thick silica, using plasma-enhanced chemical vapor deposition (PECVD) at $400^{\circ} \mathrm{C}$ (Centura P5200).

SEM images of the fabricated PhC (Fig. 3.6(a)-(c)) shows slightly deviations from the design. A clear variation in terms of length of $L_{i}, W_{i}$ and period $(\Lambda)$ is present ( $L_{i} \simeq 90 \mathrm{~nm}, W_{i} \simeq 450 \mathrm{~nm}$ and $\Lambda \simeq 231 \mathrm{~nm}$ ) when compared to the one of the design ( $L_{i}=110 \mathrm{~nm}, W_{i}=400 \mathrm{~nm}$ and $\Lambda=230 \mathrm{~nm}$ ), and can be ascribed to the difficulty of resolving the small features of the sidewall gratings by the electron beam and by optical proximity effect. The latter are even more pronounced in periodic structures 


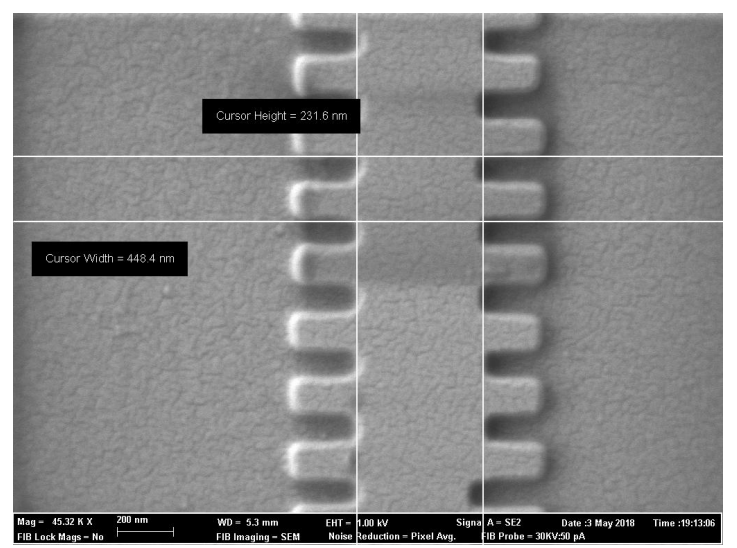

(a)

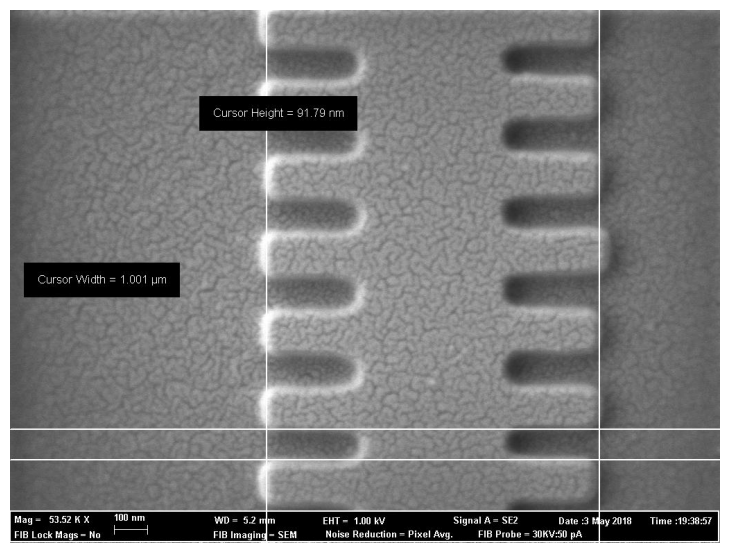

(c)

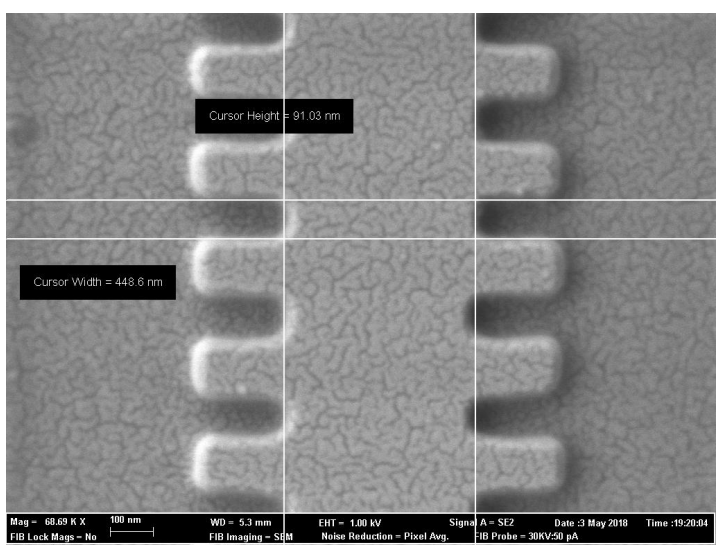

(b)

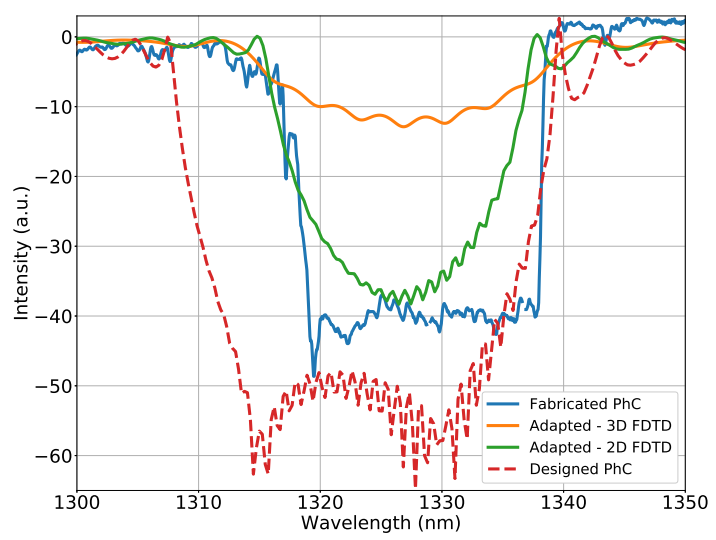

(d)

Figure 3.6: (a), (b), (c) SEM images of the fabricated PhC at NTC; (d) Comparison between original design, fabricated device and simulations, with new dimensions. The good agreement verifies the change induced by the fabrication process on $\mathrm{PhC}$. The modified dimensions are: $L_{i} \simeq 90 \mathrm{~nm}, W_{i} \simeq 450 \mathrm{~nm}$ and $\Lambda \simeq 231 \mathrm{~nm}$.

with small features due to the superposition of multiple fields during the resist exposure process.

To verify that these variations are the cause for the modified transmission response, fine meshed FDTD simulations have been performed, and the results are summarized in Fig. 3.6. The simulations show good agreement with the measured spectra; this is proof that the cause for the discrepancy between what have been measured and the design response arise from fabrication issues. 


\subsubsection{Setup}

The devices have been measured using the vertical coupling setup depicted in Fig. 3.7. The CW laser input is polarized manually in order to maximize the coupling with the input grating couplers. Between the polarizer and the input grating, a circulator has been introduced, in order to avoid any reflected light, which could interfere with the measurements or reflected back to the laser. Finally, the output fiber is connected to a power meter.
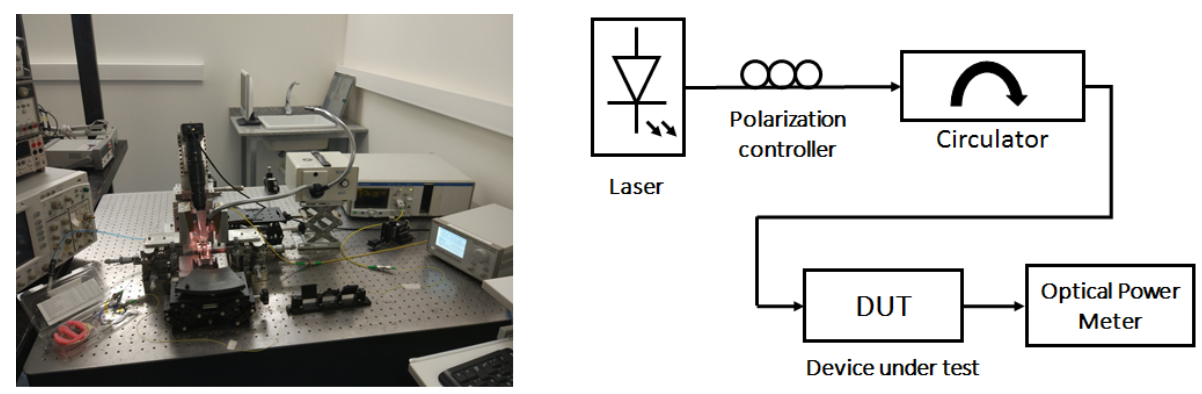

Figure 3.7: Picture and schematic of the setup used for the characterization of the slow-light structures.

\subsubsection{Results}

The measured structures have been analysed in terms of:

- Transmission Spectra

- Propagation Losses ( $\mathrm{dB} \backslash \mathrm{mm})$

- Group Index $\left(n_{g}\right)$

- Efficiency and trade-off between group index and losses

Losses and group index values are obtained via a direct interferometric method. The $\mathrm{PhCs}$ is embedded in the longer arm of an asymmetrical MZI, while a rib waveguide, of the same geometry and length, is positioned in the opposite arm as a reference. The asymmetrical MZI has an FSR of 0.57 nm (100 GHz @ $1310 \mathrm{~nm}$ ) achieved with a $\Delta L_{M Z I}$ of $800 \mu \mathrm{m}$, which allows us to obtain a fine characterisation of the photonic crystals near the PBG. Using the information resulting from the measured interference patterns, it is possible to obtain values of losses and $n_{g}$ through Eq. 3.4 
and Eq. 3.5 respectively.

$$
\begin{gathered}
P L^{P h C}(d B / m m)=\frac{\ln (|-2 *(1+R)+(4 * \sqrt{R}) /(2 *(1-R))|)}{L_{P h C}} \\
\text { with } R=10^{\frac{-\left(I_{\max }(d B)-I_{\min }(d B)\right)}{10}} \\
n_{g}^{P h C}=\frac{\lambda_{\min } \lambda_{\max }}{2\left(\lambda_{\min }-\lambda_{\max }\right) L_{P h C}}+\frac{L_{P h C}-\Delta L_{M Z I}}{L_{P h C}} n_{g}^{W G}
\end{gathered}
$$

The propagation losses $\left(P L^{P h C}\right)$, in terms of $\mathrm{dB} / \mathrm{mm}$, are obtaining using the maxima and minima power values $(\mathrm{dB})$ of the interference pattern created by the MZI. Instead $n_{g}$ is determined by the relative positioning $\left(\lambda_{\min }\right.$ and $\left.\lambda_{\max }\right)$ of these maxima and minima versus wavelength. Figure 3.8 shows an example of an analysis

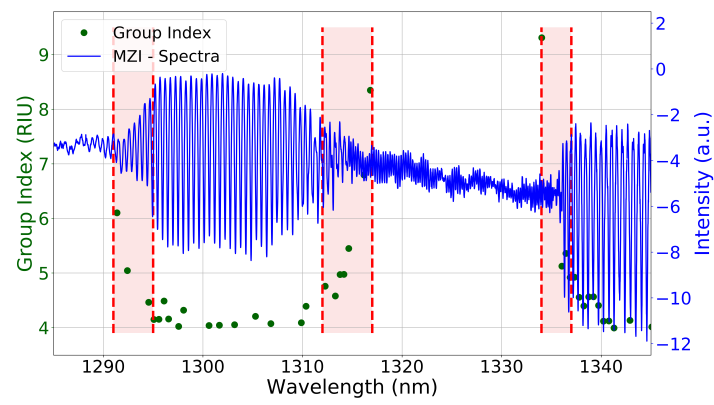

(a)

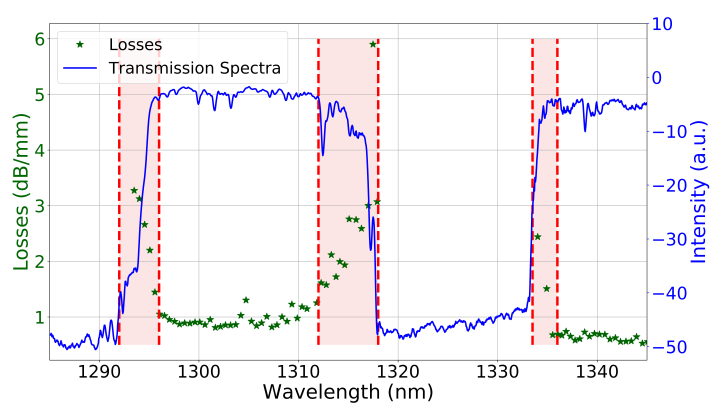

(b)

Figure 3.8: (a) Example MZI spectrum with corresponding calculated values of group index, up to $n_{g} \simeq 9$-10 near the slow-light regions, which are highlighted by the red areas. (b) Example of transmission spectra and corresponding values of propagation losses. Similarly to $n_{g}$, the losses increases significantly when approaching the slow-light regions.

where it is possible to observe the slow-light regions highlighted by the red bands and the calculated values of group index and propagation losses, together with the corresponding interferometric response and transmission spectra.

Fig. 3.8(a) shows clearly the group index increases when approaching the edges of the band gaps (red-areas) up to values of $n_{g} \simeq 9$-10. It's important to point out that, as the value of $n_{g}$ is proportional to the FSR of each resonance, it can be challenging to observe their progression. In order to observe it in detail, the FSR of the MZI should be properly increased, despite resulting in a decreased resolution of the quantities under investigation. 
In the same way, Fig. 3.8(b) gives values concerning the behaviour of the propagation losses in the slow-light regions. As expected, they increment significantly, up to $4-6 \mathrm{~dB} / \mathrm{mm}$, when approaching the PBG. Another way to qualitatively observe this mechanism, is by examining the extinction ratio of the MZI's resonances in the slow-light regions (Fig. 3.8(a)), which progressively decrease approaching nearly zerd ${ }^{3}$ (see Eq. 3.4).

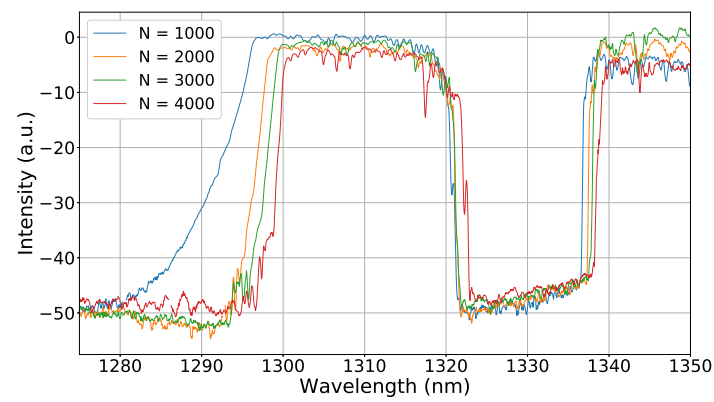

(a)

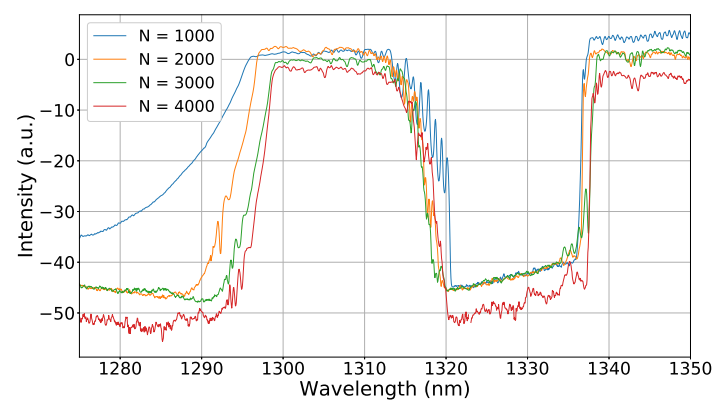

(c)

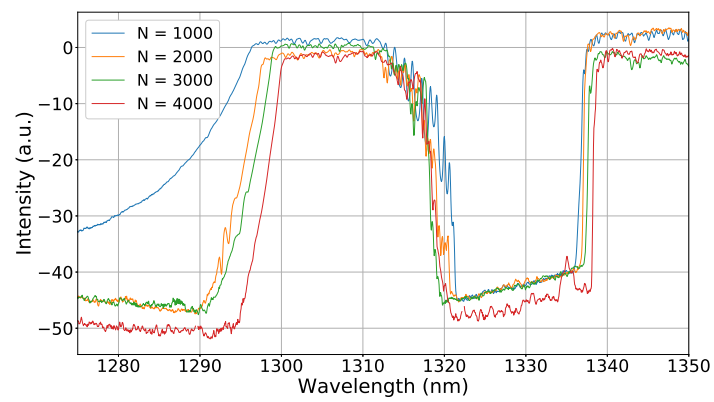

(b)

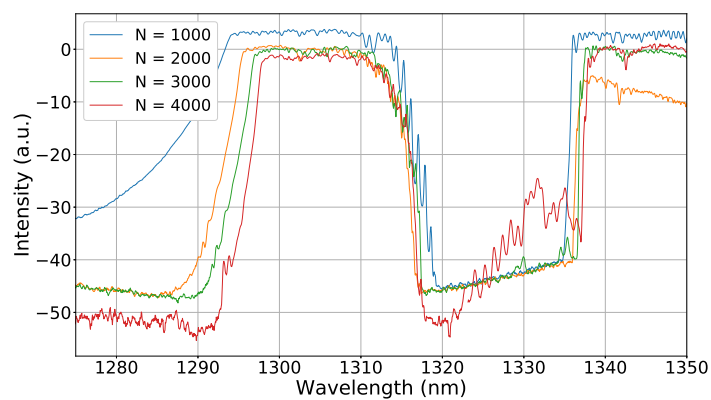

(d)

Figure 3.9: Normalized transmission spectra of the measured PhC: (a) No apodization, (b) Blackman, (c) Raised Sine and (d) Linear. Four different length were measured (1000, 2000, 3000 and 4000 periods) with a $\Lambda \simeq 230 \mathrm{~nm}$.

The measured structures have been fabricated in four different set of number of periods, namely $1000,2000,3000$ and 4000, with a $\Lambda \simeq 231 \mathrm{~nm}$ leading to the respective lengths of 231, 462, 693 and $924 \mu \mathrm{m}$. This approach was chosen to account for deviations in the design and especially to observe wheter the length of the $1 \mathrm{D}-\mathrm{PhC}$ would affect the performances in terms of losses and group index $\left(n_{g}\right)$.

\footnotetext{
${ }^{3}$ The ER should reach theoretically "0". However, due to fabrication imperfection and defects of the PhC, this limit can not be achieved.
} 
As illustrated in Fig. 3.9(a)-(d), the spectra shows similar features across all four lengths for each one of the four different designs. Indeed, the PBG location is consistent across the four lengths of each apodisation type. It is important to reiterate that the measured device shows a noticeable deviation from the simulated design, however this discrepancy is maintained constant across the different designs. This issue will require a future process of optimization in order to adapt the design to the fabrication process, allowing to increase the readiness and yield of such devices. Figure 3.9 (a) depicts the transmission spectra of the unapodized photonic crystal. The edge of the first band is located at $\sim 1337 \mathrm{~nm}$, the second is located $\sim 1322-1323$ $\mathrm{nm}$ and the third band is the one that shows a progressive redshift when the number of periods is increasing, leading to a movement of the edge from $1295 \mu \mathrm{m}$ to 1300 $\mu \mathrm{m}$. This behaviour can also be noticed in the tapered versions (Fig. 3.9(b)-(d)). It may be appointed to a lower confinement of the third mode of the photonic crystal, where 1000 periods are not enough to have a transition comparable to the other two. Therefore an increased number of period is required to achieve a higher reflectivity. Indeed, the edge steepness between 1D-PhC with 1000 and 2000 periods is readily noticeable.

It is interesting to notice the excellent performances of the measured $1 \mathrm{D}-\mathrm{PhC}$ in terms of extinction ratio (ER): featuring up to 45-50 dB ER depth.

The application of the apodisation function to the $\mathrm{PhC}$ determines the changes in the transmission spectra:

- Steepness of the first band transition.

- Higher FP resonances at the edge of the second band

- Blue-shift of the $2^{\text {nd }}$ and $3^{\text {rd }}$ band.

The first band shows a strong suppression of the Fabry-Perot resonances, which determines the creation of a very steep entrance into the PBG. This change allows us to achieve a fast transition from "bright" to "dark", which occurs in the span of 1-2 nm. The steepness clearly increases when compared to the untapered 1D-PhC, where the transition occurs across a larger span of $\sim 3 \mathrm{~nm}$.

Surprisingly, the application of tapering functions produces a worsening performances of the second band edge. In fact, these areas of the spectra exhibit an increase in magnitude of the Fabry-Perot resonances. This asymmetrical behaviour in the two different parts of the PBG has been reported in fiber bragg gratings 
(FBG) applications [31, and it is related to the use of a "non-zero dc" apodisation, which induces a variation into the average refractive index of the device. This issue will be further investigated in Sec. 3.4.

Another effect of the implementation of an apodisation function concerns the position of the second and third band edges. This offset, which can be quantified over $\sim 2-5 \mathrm{~nm}$, depends on the type of apodisation function: Raised Sine and Linear apodization induce an offset of up to $5 \mathrm{~nm}$ (Fig.3.9(c)-(d)), while with Blackman (Fig $3.9(\mathrm{~b}))$, the deviation is slightly smaller $(\sim 2 \mathrm{~nm})$.

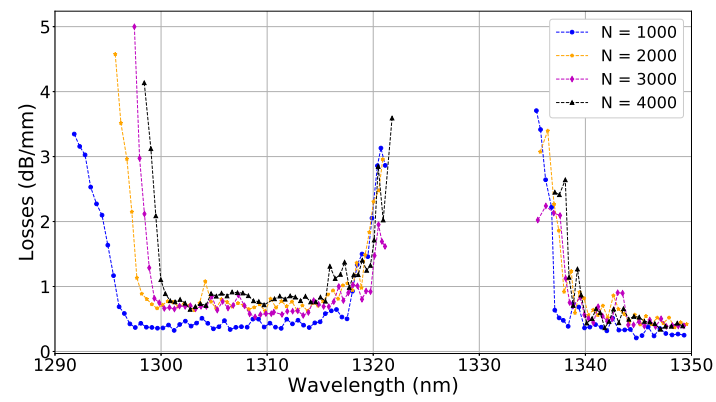

(a)

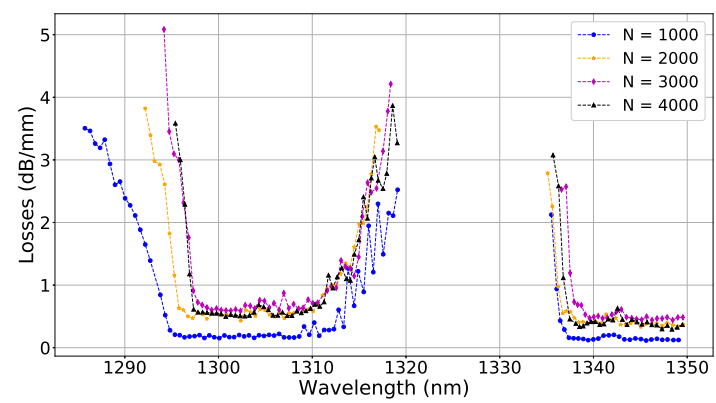

(c)

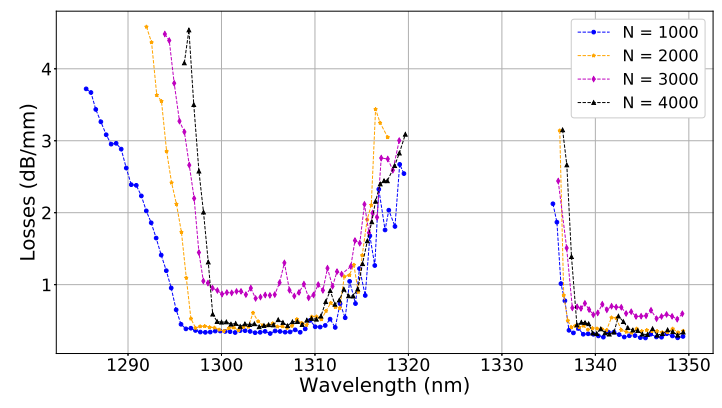

(b)

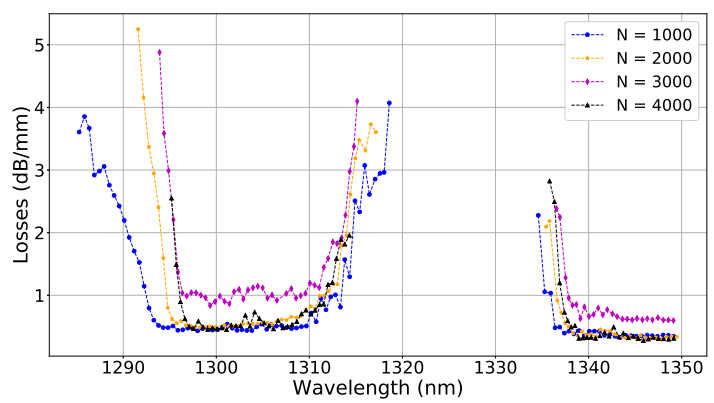

(d)

Figure 3.10: Propagation losses of the measured spectra: (a) No apodization, (b) Blackman, (c) Raised Sine and (d) Linear. Four different length were measured (1000, 2000, 3000 and 4000 periods) with a $\Lambda \simeq 231 \mathrm{~nm}$.

The first quantity of interest to evaluate the performances of the $1 \mathrm{D}-\mathrm{PhCs}$ is the propagation losses, which are summarized in Fig. 3.10, for both untapered and tapered PhC. As shown previously in Fig. 3.8(b), the loss value increases significantly when approaching the band edge. Although Bloch-modes (or resonant slow light modes) are theoretically loss less [30], they cannot be in practice due to the intrinsic 
trade-off existing between losses and group index [30, 32, 33] in photonic crystals inherent to positional disorder and fabrication imperfections.

Two distinct areas can be highlighted: the slow and fast light regions. The fast light losses are areas where no dispersion is observed and the group velocity $\left(v_{g}\right)$ is not reduced. For all four cases (STD, BKM, RS and LN) these zones are in three ranges of wavelength: $\sim 1300-1310 \mathrm{~nm}$ and $\sim 1340-1350 \mathrm{~nm}$. In these intervals the propagation losses are, on average, of $\sim 0.7-0.8 \mathrm{~dB} / \mathrm{mm}$. This value is slightly than the $0.2 \mathrm{~dB} / \mathrm{mm}$ a standard silicon rib waveguide [34. We retain that this increase is induced by the $1 \mathrm{D}-\mathrm{PhC}$ itself exhibiting artificial periodic roughness, namely, the sidewall gratings. Another theoretical source of losses could be the optical mismatch caused by the transition from the standard waveguide and the corrugated waveguide [27, 30]. However, this contribution should be minimized with the implementation of an apodisation function. Instead, as depicted by Fig. 3.10(b)-(d), in these intervals the propagation losses for the tapered $1 \mathrm{D}-\mathrm{PhCs}$ remain constant, so it can be assumed that the contribution induced by optical mismatch can be considered negligible.

The slow-light area, i.e. the regions in which the group velocity $\left(v_{g}\right)$ is reduced, are located in the range of wavelength close to the edge of the bands. The losses of 1D$\mathrm{PhC}$ increases significantly, up to $4-5 \mathrm{~dB} / \mathrm{mm}$. These higher levels of propagation losses are caused mainly by the increasing backscattering reflection caused by the $\mathrm{PhC}$ itself when approaching the band edge.

The other quantity of interest is the group index $\left(n_{g}\right)$ and the results are summarized in Fig. 3.11. Like the propagation losses, $n_{g}$ significantly increases when approaching the band edges.

Similarly to the propagation losses case, the graphs can be divided between fast and slow light regions, where the range of wavelength are the same. The fast light areas of the spectrum shows values of group index close to the value of a $400 \mathrm{~nm}$ wide silicon rib waveguide that is $n_{g}^{W G} \simeq 3.74$. In these regions, the light is not slowed down, determining standard propagation.

Close to the edge of the $1^{\text {st }}$ and $2^{\text {nd }}$ bands, values of $n_{g} \simeq 9$-10 have been measured across all four different designs. Conversely, for the third band we have not been able to measure values higher than 5 . This may be explained by the fact the third 


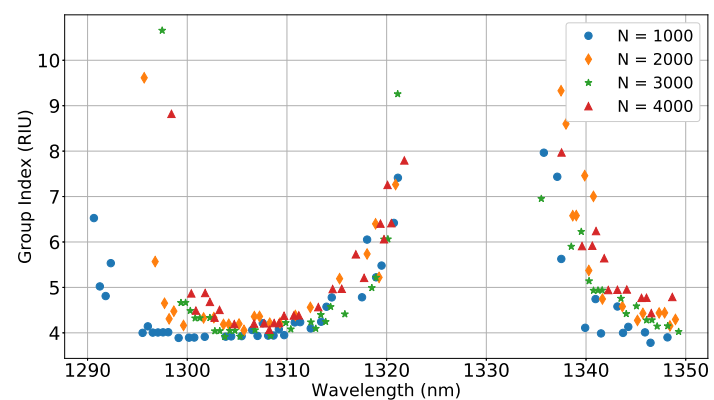

(a)

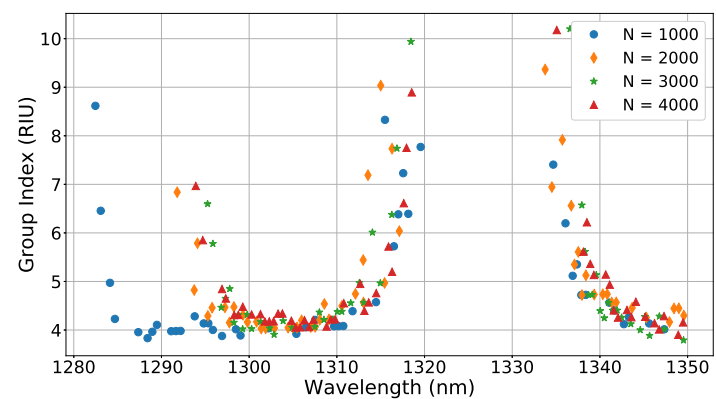

(c)

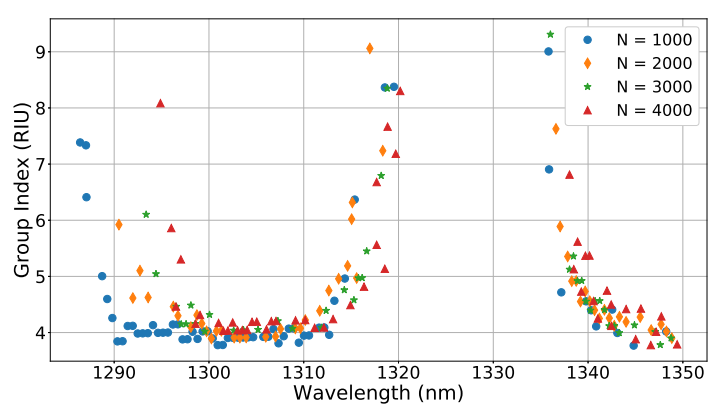

(b)

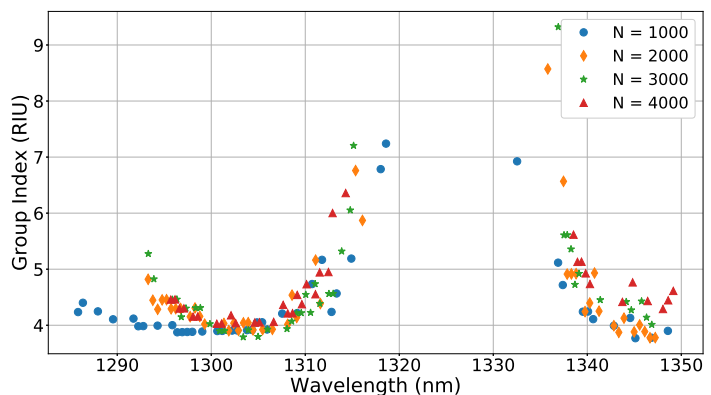

(d)

Figure 3.11: Group index $\left(n_{g}\right)$ of the measured spectra: (a) No apodization, (b) Blackman, (c) Raised Sine and (d) Linear. Four different length were measured (1000, 2000, 3000 and 4000 periods) with a $\Lambda \simeq 231 \mathrm{~nm}$.

mode is less confined and does not "feel" the periodic index contrast as much as the first two modes.

As already mentioned, when working with these photonic crystals, a trade-off between propagation losses and group index of the device has to be taken into account. Hence, in order to have a more complete overview of the performances, an in-depth analysis of their relation has to be made. To facilitate the comparison between the different apodisation cases, a figure of merit (FOM) has to be established, putting into direct relation the losses with the corresponding value of group index and taking as a reference the work by [35] and that the relation between losses and $n_{g}$ is linear, for value of $n_{g}<25$. The FOM is obtained performing a linear regression of the data, where its slope is the 1D-PhC's FOM, expressed as dB/RIU [36]. Physically, the FOM is the penalty, in terms of $\mathrm{dB}$ (normalized for $1 \mathrm{~mm}$ ), for each nominal increase of the group index. 
The results are summarized in Fig. 3.12 and Table 3.1

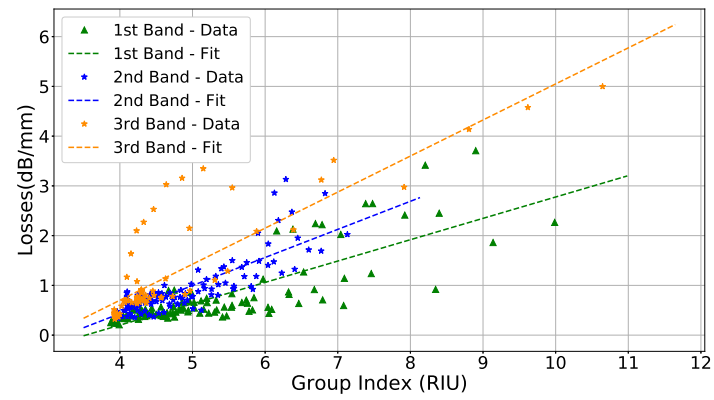

(a)

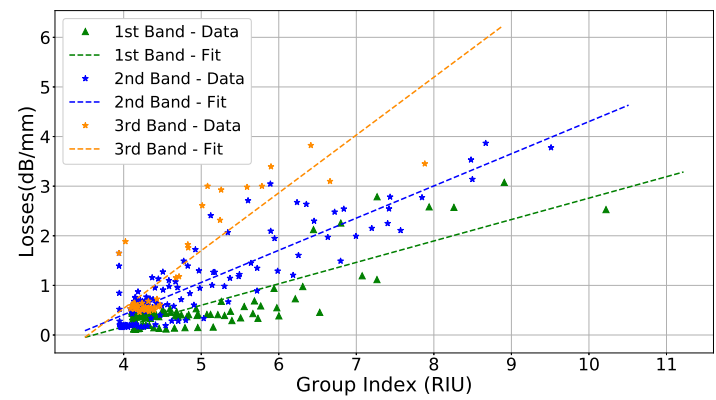

(c)

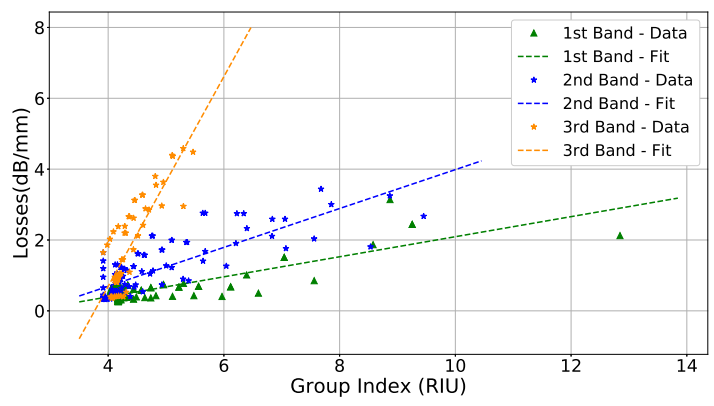

(b)

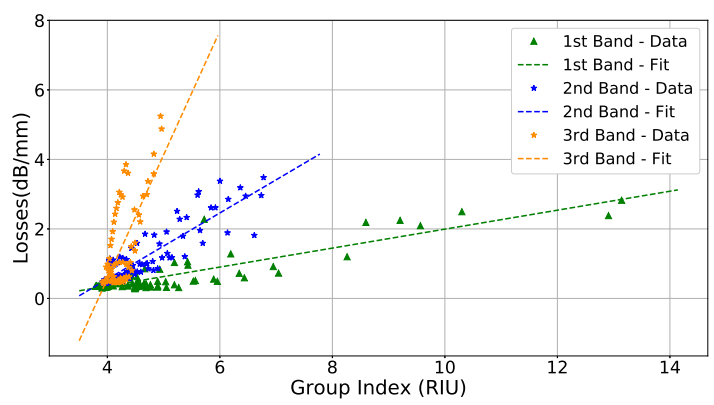

(d)

Figure 3.12: The losses and $n_{g}$ values has been related and using a linear interpolation the FOM for each one of the bands as been calculated.(a) No apodization, (b) Blackman, (c) Raised Sine and (d) Linear.

\begin{tabular}{c|c|c|c|c}
\hline & $\begin{array}{c}\text { No Taper } \\
(\mathrm{dB} / \mathrm{RIU})\end{array}$ & $\begin{array}{c}\text { Blackman } \\
(\mathrm{dB} / \mathrm{RIU})\end{array}$ & $\begin{array}{c}\text { Raised Sine } \\
(\mathrm{dB} / \mathrm{RIU})\end{array}$ & $\begin{array}{c}\text { Linear } \\
(\mathrm{dB} / \mathrm{RIU})\end{array}$ \\
\hline $1^{\text {st }}$ band & 0.43 & 0.28 & 0.44 & 0.27 \\
$2^{\text {nd }}$ band & 0.56 & 0.55 & 0.65 & 0.95 \\
$3^{\text {rd }}$ band & 0.73 & 2.96 & 1.17 & 3.56 \\
\hline
\end{tabular}

Table 3.1: Values of FOM for each design and each band

Using as a reference the values of Table 3.1, it is possible to extrapolate immediately a trend for the FOM values. Taking as an example the untapered $\mathrm{PhC}$ (Fig. $3.12(\mathrm{a})$ ), it can be observed that the FOM decrease (the slope of the interpolating straight line increases) in a straightforward manner, FOM $1_{1^{s t}}<F O M_{2^{\text {nd }}}<F O M_{3^{\text {rd }}}$, 
when the number of the band increases. This pattern can be observed also in the tapered cases, as shown in Fig. 3.12(b)-(d). According to our interpretation, this behaviour is directly connected to the confinement of each guided mode, which decreases progressively when the band order increases. Quantitatively this can be evaluated looking at the value of $n_{\text {eff }}$ for each periodic mode, because such value is directly correlated to the level of confinement of each optical mode. Indeed, as the band number increases $n_{\text {eff }}$ of the related periodic mode decreases as summarized by Table 3.2. Therefore, as the number of the band increases, the loss penalty for each unit of refractive index increases, determining that each band is progressively less efficient.

\begin{tabular}{c|c|c}
\hline$n_{\text {eff }}^{1 s t}$ & $n_{\text {eff }}^{2 n d}$ & $n_{\text {eff }}^{3 r d}$ \\
\hline \hline 2.88 & 2.79 & 2.75 \\
\hline
\end{tabular}

Table 3.2: Values of effective refractive index $\left(n_{e f f}\right)$ for each one of the optical mode of the $\mathrm{PhC}\left(\Lambda=230 \mathrm{~nm}, L_{i}=120 \mathrm{~nm}\right.$ and $\left.L_{e}=110 \mathrm{~nm}\right)$. The values are calculated using the PWE method.

More interesting are the values concerning the apodized $\mathrm{PhCs}$, as each type of scheme affects differently the performances of each individual band (as summarised in Table 3.1]. These changes can summarised as follows:

- Blackman (BKM): the first band shows an increase in efficiency of a few decimals, the second band does not seem to be affected and the third band performances significantly worsen with a losses-to-RIU ratio increase of nearly four times.

- Raised Sine (RS): the first and second band efficiency do not variate from the untapered case, the third band worsens as well, but by a lower magnitude when compared to the other apodisation schemes.

- Linear (LN): The first band increases its efficiency, with a magnitude comparable to BKM configuration, however it worsens the FOM of the second band (few decimals) and the third band up to nearly five times. 
Overall, the first band shows a slight improvement when BKM and LN functions are applied. The second band is not affected and worsens only slightly when applying the LN taper. Yet, higher variance and unexpected worsening is measured when looking at the overall performance of the third band.

We speculate that because the third mode is TM-like mostly located in the wider parts of the photonic crystal, the apodisation scheme acting on the sidewall width of the 1D-PhC has profound effects on its performances. Thus such mode when transitioning from the waveguide mode to the slow-light mode, and vice versa, has really low confinement when compared to the untapered version. As a result, the propagation losses increases significantly as reflected in the corresponding figure of merit.

\subsubsection{Results - Partial Tapers}

In order to improve the performances of the apodizated $\mathrm{PhC}$, the use of a partial taper on both sides of the structure (input/output) has been taken into account. This means that the apodization has been applied only to part of the $1 \mathrm{D}-\mathrm{PhC}$ and not on its entirety. Using as a reference the 4000 periods structure, two distinct combination with 750 and 1500 tapered repetitions, on each side, have been fabricated and measured. This layout has been applied for all three different apodisation functions. Unlike in Sec. 3.3.2, only the FOM results are shown, to avoid any redundancy due to their resemblance to what presented in Sec. 3.3.2. However, these graphs can be found in Appendix $\mathrm{A}$ for the sake of completeness.

\begin{tabular}{c|c|c|c}
\hline & $\begin{array}{c}\text { 1st Band } \\
(\mathrm{dB} / \mathrm{RIU})\end{array}$ & $\begin{array}{c}\text { 2nd Band } \\
(\mathrm{dB} / \mathrm{RIU})\end{array}$ & $\begin{array}{c}\text { 3rd Band } \\
(\mathrm{dB} / \mathrm{RIU})\end{array}$ \\
\hline Untapered & 0.43 & 0.57 & 0.72 \\
$\mathrm{~N}=750$ & 0.12 & 0.81 & 1.53 \\
$\mathrm{~N}=1500$ & 0.44 & 0.63 & 3.37 \\
$\mathrm{~N}=2000$ & 0.28 & 0.54 & 2.96 \\
\hline
\end{tabular}

Table 3.3: FOM values for the progressive Blackman layout. Full tapered and untapered versions are present for completeness. 


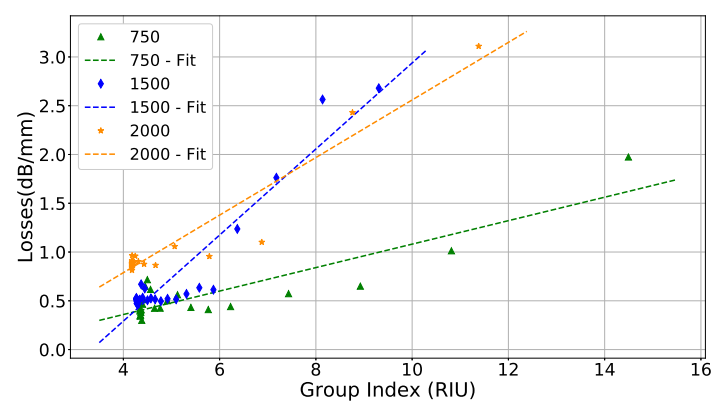

(a)

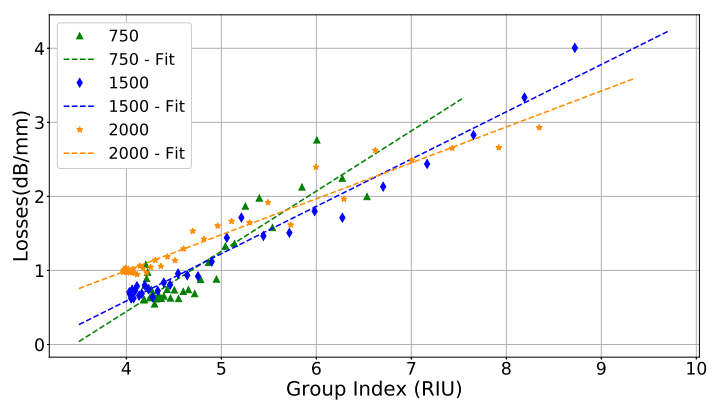

(b)

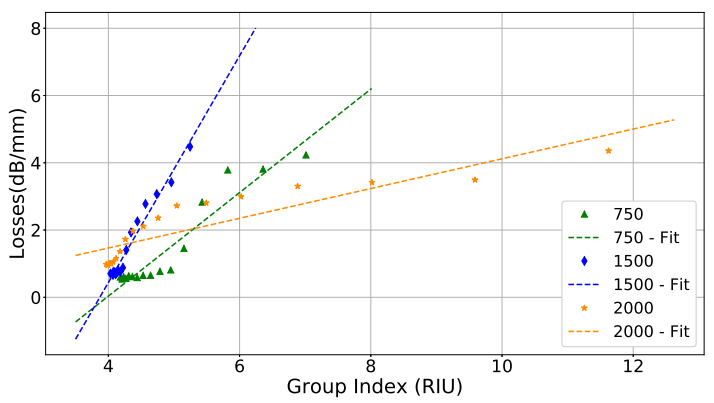

(c)

Figure 3.13: FOM values for the progressive Blackman layout: (a) $1^{\text {st }}$ band, (b) $2^{\text {nd }}$ band and $3^{\text {rd }}$ band

The results of the partial BKM tapers are summarized in Fig. 3.13 and Table 3.3 . Compared to the other cases, the first band shows a noticeable improvement when a taper featuring $\mathrm{N}=750$ periods is applied, reaching an efficiency of $0.12 \mathrm{~dB} / \mathrm{RIU}$. Interestingly for $\mathrm{N}=1500$ the performances significantly decrease. According to our interpretation, this unexpected result is caused by the fabrication of the $1 \mathrm{D}-\mathrm{PhC}$ itself. In the future, further samples should be fabricated and measured in order better characterize this specific structure. The values of the second band show no clear improvements, and the measured values for $\mathrm{N}=750$ and $\mathrm{N}=1500$ are really similar to the untapered and full tapered versions. Fundamentally, the efficiency of the second band seems not to be affected by the use of any apodisation. A possible explanation is that, being the TE-like mode of the second band located in the narrow part of the $1 \mathrm{D}-\mathrm{PhC}$ and that the tapering only affects the sidewall gratings, the apodisation does not influence this specific mode. The third band shows a clear worsening in terms of efficiency even when a partial taper is applied. As previously discussed, this trend can be justified by the shape of the third periodic 
mode, which is located in the wider part of the photonic crystal. Thus, when a full or partial apodisation is applied, the losses caused by the overall lower confinement of the TM-like mode significantly increases, producing higher FOM values.

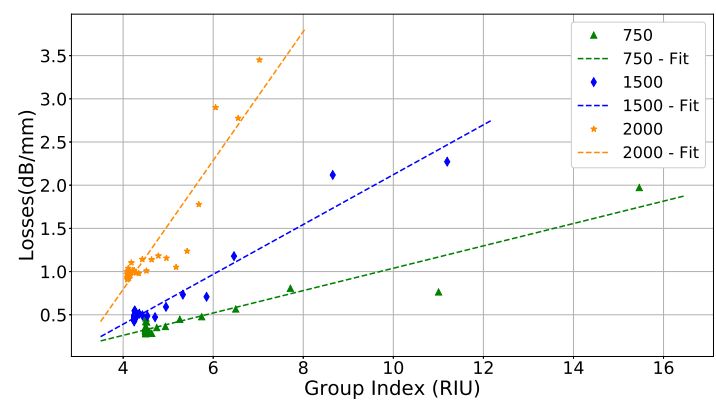

(a)

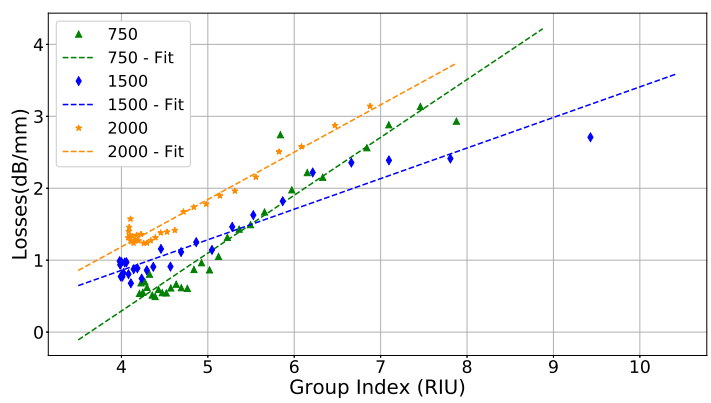

(b)

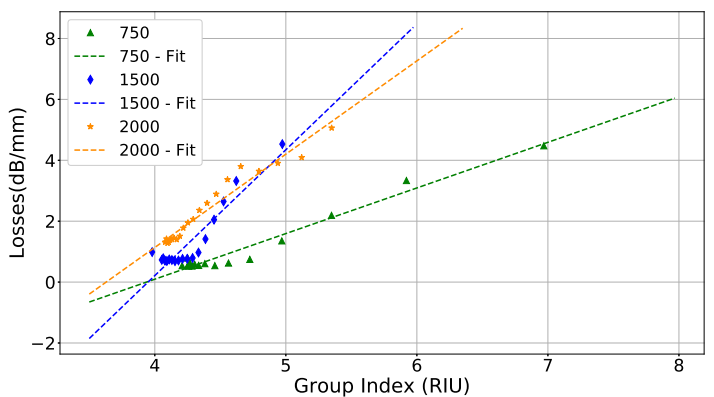

(c)

Figure 3.14: FOM values for the progressive Raised Sine layout: (a) $1^{\text {st }}$ band, (b) $2^{\text {nd }}$ band and $3^{\text {rd }}$ band

\begin{tabular}{c|c|c|c}
\hline & $\begin{array}{c}\text { 1st Band } \\
(\mathrm{dB} / \mathrm{RIU})\end{array}$ & $\begin{array}{c}\text { 2nd Band } \\
(\mathrm{dB} / \mathrm{RIU})\end{array}$ & $\begin{array}{c}\text { 3rd Band } \\
(\mathrm{dB} / \mathrm{RIU})\end{array}$ \\
\hline Untapered & 0.43 & 0.57 & 0.72 \\
$\mathrm{~N}=750$ & 0.12 & 0.80 & 1.50 \\
$\mathrm{~N}=1500$ & 0.28 & 0.42 & 4.13 \\
$\mathrm{~N}=2000$ & 0.43 & 0.64 & 1.16 \\
\hline
\end{tabular}

Table 3.4: FOM values for the progressive Raised Sine layout. Full tapered and untapered versions are present for completeness.

The results of the partial RS tapers are presented in Fig. 3.14 and Table 3.4 . As the previous case, the best layout for the first band turns out to be the par- 
tial taper with input and output of 750 periods. With regards to the second band, the best measured FOM is when the number of period is $\mathrm{N}=1500$. However, as previously described, no enhancement of performances is expected when applying an apodisation to the TE-like mode of the second band. Moreover, observing Fig. 3.15(b), it is possible to observe how the curves looks quite similar. These small deviations can arise from defects and fluctuations in the fabrication process of each 1D-PhC. The third band shows a trend similar to the BKM case, where the overall performances of the TM-like mode, compared to the untapered 1D-PhC are worst.

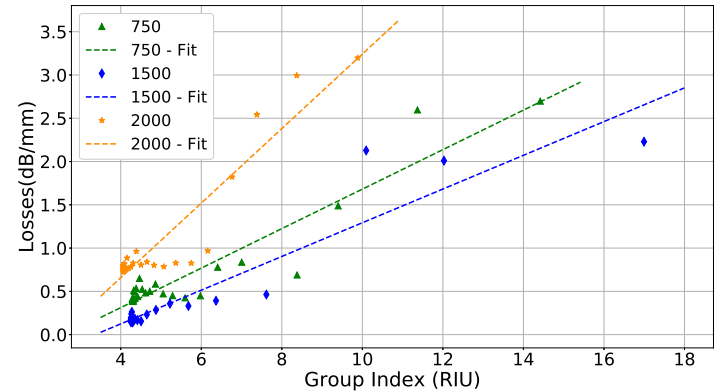

(a)

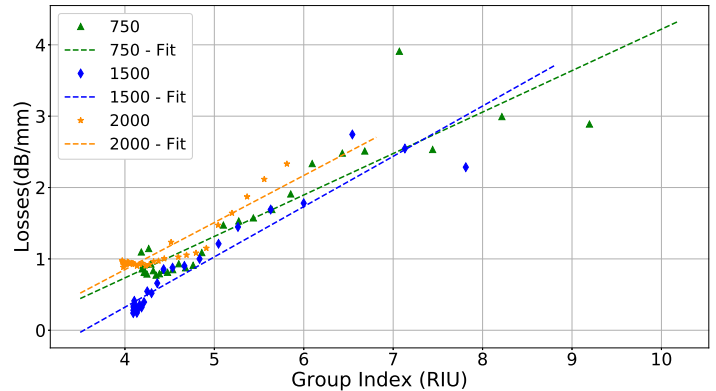

(b)

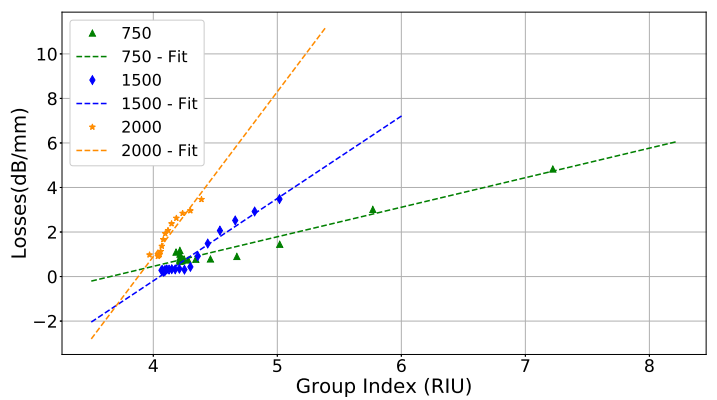

(c)

Figure 3.15: FOM values for the progressive Raised Sine layout: (a) $1^{\text {st }}$ band, (b) $2^{\text {nd }}$ band and $3^{\text {rd }}$ band

The results of the partial LN tapers are summarized in Fig. 3.15 and Table 3.5 These layouts show similar trends than those we have just discussed: the second band shows the same trend across the three versions (Fig. 3.15(b)), and the third increases FOM values as the number of apodised periods increments (Fig. 3.15(c)). The main variations can be observed in the first band performances, where the FOMs of the tapered $1 \mathrm{D}-\mathrm{PhC}$ are reduced equally when compared to the untapered version. 


\begin{tabular}{c|c|c|c}
\hline & $\begin{array}{c}\text { 1st Band } \\
(\mathrm{dB} / \mathrm{RIU})\end{array}$ & $\begin{array}{c}\text { 2nd Band } \\
(\mathrm{dB} / \mathrm{RIU})\end{array}$ & $\begin{array}{c}\text { 3rd Band } \\
(\mathrm{dB} / \mathrm{RIU})\end{array}$ \\
\hline Untapered & 0.43 & 0.57 & 0.72 \\
$\mathrm{~N}=750$ & 0.22 & 0.58 & 1.31 \\
$\mathrm{~N}=1500$ & 0.19 & 0.70 & 3.69 \\
$\mathrm{~N}=2000$ & 0.27 & 0.95 & 3.56 \\
\hline
\end{tabular}

Table 3.5: FOM values for the progressive Linear layout. Full tapered and untapered versions are present for completeness.

In conclusion, this layouts affect each specific band in different ways: the $1^{\text {st }}$ band is the one which shows the more improvements in terms of efficiency, up to values as low as $0.12 \mathrm{~dB} / \mathrm{RIU}(\mathrm{N}=750$ for BKM and $\mathrm{LN})$. The $2^{\text {nd }}$ band does not present any noticeable improvement from the untapered version. However, the most interesting consequences have been measured in the $3^{\text {rd }}$ band, where up to four times, higher losses have been measured.

\subsection{Double Taper Apodization}

As shown in the previous section, the first layout creates undesired Fabry-Perot resonances at the edge of the second band, which influence the transmission spectra in an unwanted way, but mainly it could reduce the overall operation efficiency of the $\mathrm{PhC}$ in terms of $\mathrm{dB} / \mathrm{RIU}$.

The reason for this kind of behaviour have to be searched into the nature of the apodisation itself. According to [37], in the case of non-zero apodisation function (such in our case: Linear, Raised Sine, Blackman) the entire photonic crystal resonance is shifted slightly to longer wavelength compared to the untapered version. This is due to the increase in the space-averaged effective index of refraction, caused by the apodisation. Nonetheless, the region in the centre of the grating, where the space-averaged index of refraction is increased the most, has its resonance shifted the farthest, in relation to the other areas of the photonic crystal. Thus, there is a frequency region near the $2^{\text {nd }}$ band of the grating resonance where the grating edges are near their "local" Bragg resonances, but the centre of the photonic crystal is not. 
This gives, as a result, the undesired Fabry-Pérot resonances at the edge of the $2^{\text {nd }}$ band that we measured. Qualitatively, the sides of an apodizated photonic crystal behave as partially reflecting mirrors, and the centre as a transparent region.

In order to solve this issue and compensate for this Fabry-Perot resonances, a new solution, similar to one used in FBG applications [31, 38], has been designed featuring a double apodisation function. This configuration would compensate for this kind of behaviour induced by non-zero distributions, using a nearly zero-dc approach.

In the following sections the design will be studied and analysed, and then using the tools introduced in the previous sections, the experimental results will be analysed and discussed.

\subsubsection{Double Taper Design}

As reported previously, the reason for the presence of Fabry-Perot resonances at the edge of the second band is caused by the spatially variying effective refractive index of each section, which is determined by the width of the narrow and wide section $\left(\Lambda=230 \mathrm{~nm}, L_{i}=120 \mathrm{~nm}\right.$ and $\left.L_{e}=110 \mathrm{~nm}\right)$ of the 1D-PhC. Fig. 3.16(a) shows effective index change $\left(\Delta \mathrm{n}_{e f f}\right)$ induced by the apodised $1 \mathrm{D}-\mathrm{PhC}$ for each one of the profile under investigation (Blackman, Raised Sine and Linear). We can observe how the average refractive index contrast $(\Delta \mathrm{n})$ is well above the zero value, which is, in our case, the effective refractive index of a $400 \mathrm{~nm}$ rib waveguide. This latter waveguide is used as the input waveguides to the $1 \mathrm{D}-\mathrm{PhC}$.

In order to compensate for these Fabry-Perot resonances and trying to minimize the changes on the $1 \mathrm{D}-\mathrm{PhC}$ layout, it was decided to reduce the averaged $\Delta n_{e f f}$ with the introduction of a second apodisation acting on the narrower part of the 1D-PhC $\left(W_{i}\right)$. An example can be observed in Fig. 3.16(b).

It is important to point out that with this solution, it is practically impossible to achieve a perfect zero- $d c$ spatial distribution of the effective refractive index, due to the high $\Delta n_{e f f}$ induced by the wider region of the photonic crystal $(\sim 1 \mu \mathrm{m})$, therefore a trade-off is required. The target is to reduce sufficiently $\Delta n_{\text {eff }}$ in order to suppress the undesired Fabry-Perot resonances caused by the implementation of a single apodisation.

The simulated results are reported in Fig 3.17 , both in terms of transmission spectra and resulting $\Delta n_{\text {eff }}$ distribution. Figure 3.17(a)-(b) shows how shrinking the 


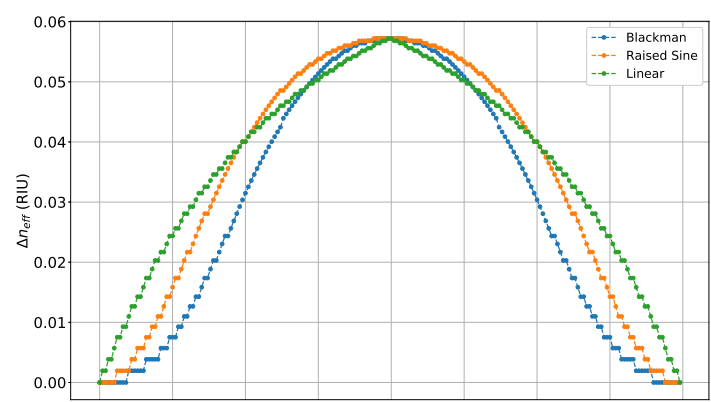

(a)

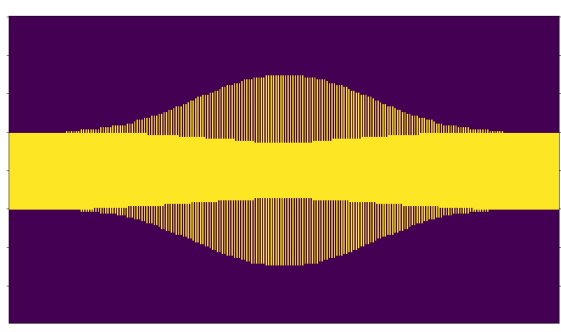

(b)

Figure 3.16: (a) The graph shows the $\Delta \mathrm{n}_{\text {eff }}$ distribution induced by the use of a single apodization functions on a 200 period $\mathrm{PhC}$. The average value is well above the reference value of the 400 $\mathrm{nm}$ slab waveguide case. (b) The image depicts a layout example $(\mathrm{N}=200)$ with double tapering functions.

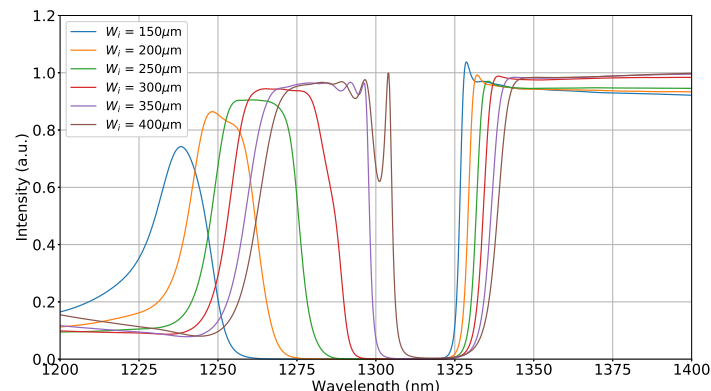

(a)

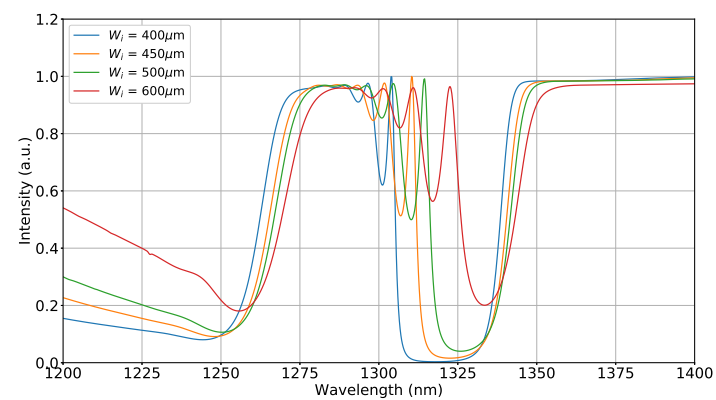

(c)

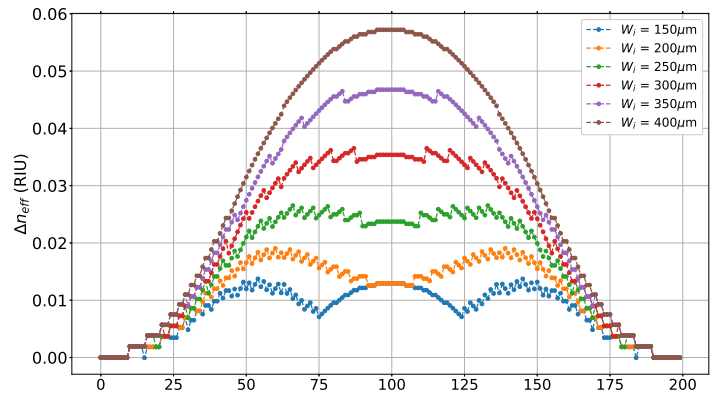

(b)

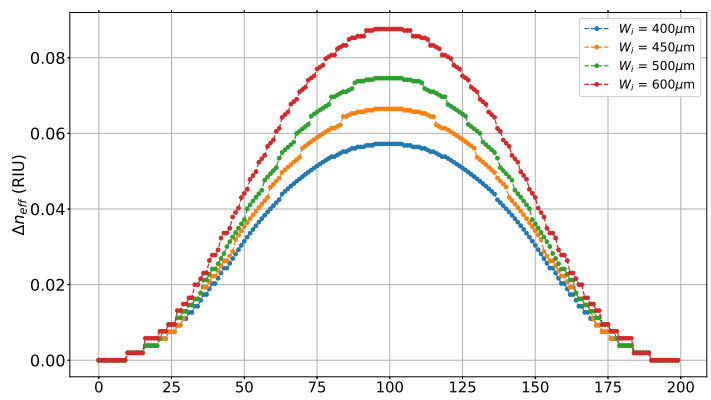

(d)

Figure 3.17: (a) Simulated transmission spectra for $W_{i} \leq 400 \mathrm{~nm}$ and (b) relative space-averaged distribution of $\Delta n_{e f f}$ for $\mathrm{N}=200$. (c) Simulated transmission spectra for $W_{i} \geq 400 \mathrm{~nm}$ and (d) relative space-averaged distribution of $\Delta n_{e f f}$.

narrow section of the waveguide $\left(W_{i}\right)$ causes the average $\Delta n_{e f f}$ to decrease, which 
directly affects the transmission spectra. The undesired resonances are suppressed when $W_{i}$ approaches $300 \mathrm{~nm}$. Moreover, a noticeable blue-shift and an increase in optical bandwidth occurs. By contrast, an increase in $W_{i}$, which translate into an increment of space-average distribution, results in stronger undesirable Fabry-Perot resonances, a red-shift of the overall spectrum and a reduction of the optical bandwidth (Fig. 3.17(c)-(d)).
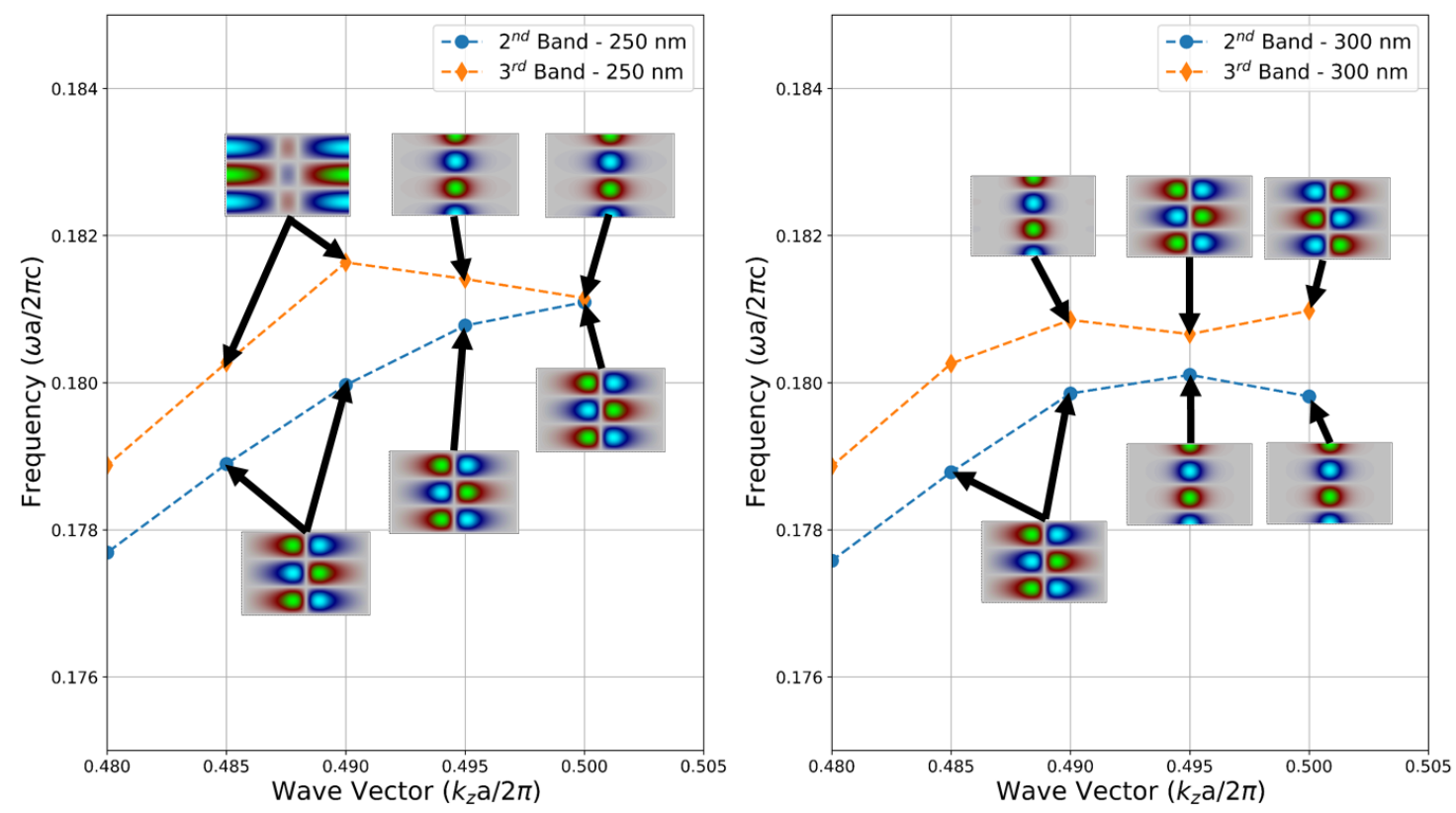

Figure 3.18: Band structure and relative optical modes for $W_{i}=250 \mathrm{~nm}$ (Left) and $W_{i}=300 \mathrm{~nm}$ (Right)

However the most interesting part is to investigate the way in which the band structure is affected and how these changes are reflected in the 1D-PhC's transmission response.

As previously discussed in Sec. 3.2 , the band structure of the 1D-PhC (using $W_{e}=1$ $\mu \mathrm{m}$ and $W i=400 \mathrm{~nm})$ is characterized by two TE-like modes $(\mathrm{m}=0$ and $\mathrm{m}=1)$ and by one TM-like mode $(\mathrm{m}=2)$ (Fig. 3.3.). The second and third band are two hybrid bands, where an anti-crossing phenomenon occurs due to periodicity added to the rib silicon waveguide. By reducing $W_{i}$ the $1 \mathrm{D}-\mathrm{PhC}$ 's band structure is progressively modified until this anti-crossing regime disappears. This occurs when $W_{i}$ changes from 300 to $250 \mathrm{~nm}$. Figure 3.18 shows how the band structure and the related 
optical modes of these two specific cases are modified.

\subsubsection{Double Taper - Measurements and Discussion}

The double taper design have been fabricated considering $W_{i}=250-300-350 \mathrm{~nm}$ and with $\mathrm{N}=3000$. The other parameters, such as the duty cycle $(\Lambda \simeq 230 \mathrm{~nm})$ and the width of the sidewall grating $\left(W_{e}=1000\right)$, have been maintained. The devices have been analysed, using the same methodology as in Sec. 3.3.2, namely:

- Transmission Spectra

- Propagation Losses (dB\mm)

- Group Index $\left(n_{g}\right)$

- Trade-off between group index and losses // Effects of the apodisation on FOM at the band edge FOM (performance)

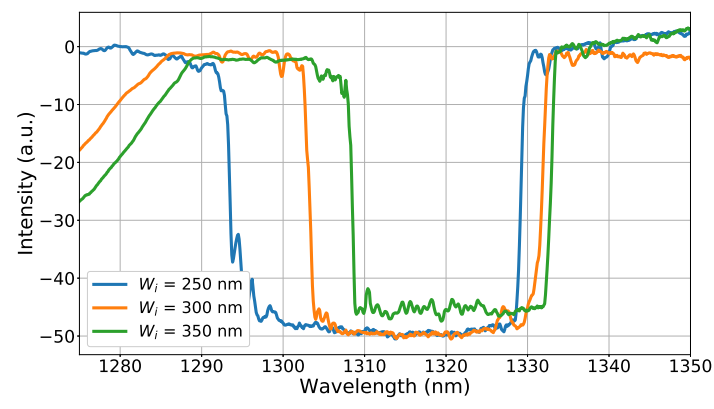

(a)

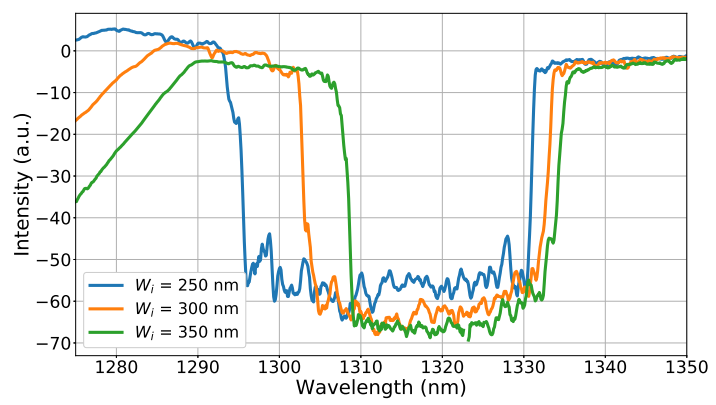

(b)

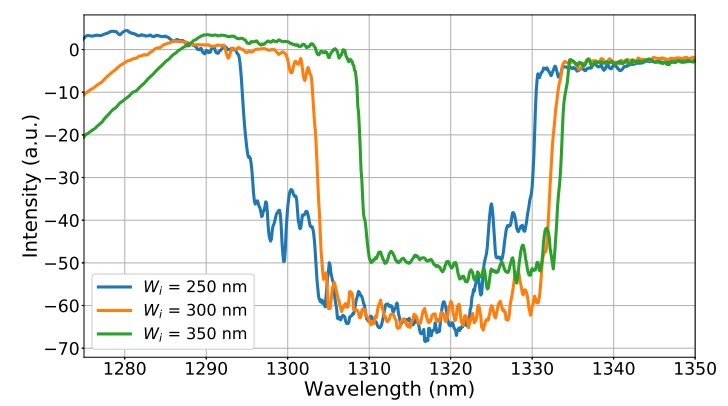

(c)

Figure 3.19: Normalized transmission spectra of the measured $\mathrm{PhC}$ with double apodisation: (a) Blackman, (b) Raised Sine and (c) Linear. Three different geometries have been measured with $W_{i}=250,300$ and $350 \mathrm{~nm}$. 
Before starting the analysis, it is important to notice that the new design causes changes the $3^{\text {rd }}$ band to blue-shift, moving to lower wavelength, moving out of the lower detection range of the measurement setup. Therefore due to limitation of the setup, it has been not possible to properly characterise that part of the spectrum. As a result, the discussion over the FOM is going to be limited to the firsts two bands.

The transmission spectra have been summarized in Fig. 3.19.

The new designs exhibit a significant suppression of the Fabry-Perot resonances on both side of the PBG, for all combinations of $W_{i}$ and types of apodisation. Additionally, the decrease of $W_{i}$ determines a direct increase in optical bandwidth of the PBG, respectively of $\sim 24-27-36 \mathrm{~nm}$ likely due to the higher index contrast. These values are reduced when compared to the simulated one.

These discrepancies are caused by fabrication deviations similar to those reported in Sec. $3.3 .2\left(L_{i} \simeq 90 \mathrm{~nm}, W_{i} \simeq 450 \mathrm{~nm}\right.$ and $\left.\Lambda \simeq 231 \mathrm{~nm}\right)$. When compared to the single taper design, an additional 30-45 nm has to be added to the designed value of $W_{i}$. These variations can be attributed to the limitations of the electron-beam tool when dealing with the small critical features (sub-100 nm) of this specific design.

Mostly importantly, one may observe the steepness of the second band edge, when compared to the single taper version (Fig. 3.9(b)-(d)). Indeed, in contrast with the single taper design the double taper design successfully creates a fast transition $(\sim 2-3 \mathrm{~nm})$, without affecting the shape of the transition of the first band edge, which remains square-shaped. Moreover, this design maintains the excellent performances in terms of extinction ratio, with values up to 45-50 dB.

Then, as said before, it is important to underline that the third band, like the second one, undergoes a blue-shift as $W_{i}$ is increased. Nonetheless, for this double taper design, it is not possible to observe the entire transition due to limited span of the tunable laser.

The propagation losses have been evaluated using the same method described in Sec. 3.3.2, and the results for all nine different configurations are summarized in Fig. 3.20, As in the case of the single taper design, the data can be divide in two main regions: fast and slow light, which are respectively far from and close to the PBG.

As expected, when approaching the band gap edge (slow-light regions) the values 


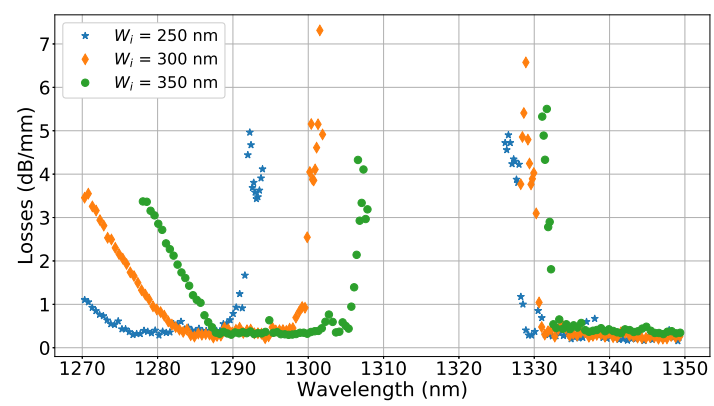

(a)

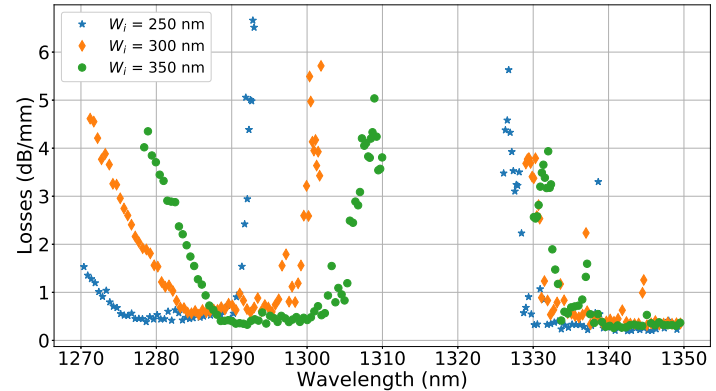

(b)

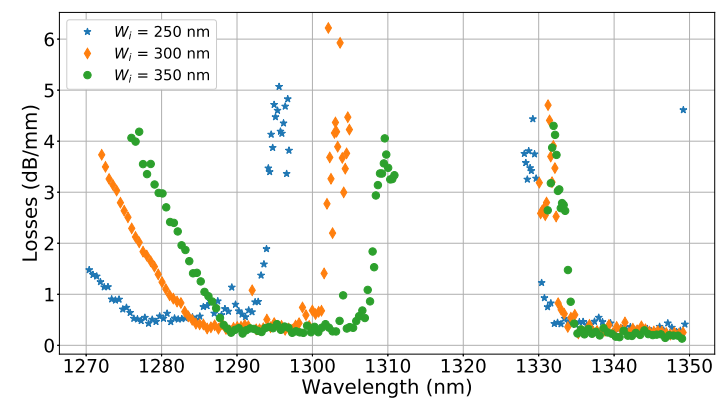

(c)

Figure 3.20: Propagation losses of the measured $\mathrm{PhC}$ with double apodisation: (a) Blackman, (b) Raised Sine and (c) Linear. Three different geometries have been measured with $W_{i}=250$, 300 and $350 \mathrm{~nm}$..

increase significantly, up to 5-7 dB/mm, which are slightly higher than those measured for the single taper design. This can be justified by the fabrication quality of the $1 \mathrm{D}-\mathrm{PhC}$ itself. Indeed, a higher sample quality allows us to measure with higher precision inside the PBG.

The remaining fast-light regions have propagation losses values slightly lower $(\sim 0.4$ $0.5 \mathrm{~dB} / \mathrm{mm}$ ) when compared to the ST design. Again, we believe that this is caused by the better quality of the samples. Whilst these values are still higher than the standard rib silicon waveguide, which have optical losses around $0.2 \mathrm{~dB} / \mathrm{mm}$ [39], we know that they are generated by the artifical sidewall roughness inherent to the $1 \mathrm{D}-\mathrm{PhC}$ itself.

The second quantity of interest is $n_{g}$, as summarized in Fig. 3.21.

Close to the band edges of the $1^{\text {st }}$ and $2^{\text {nd }}$ band, $n_{g}$ reaches $\sim 15$. It is clear that the implementation of a double taper configuration allows us to have a $1 \mathrm{D}-\mathrm{PhC}$ with 


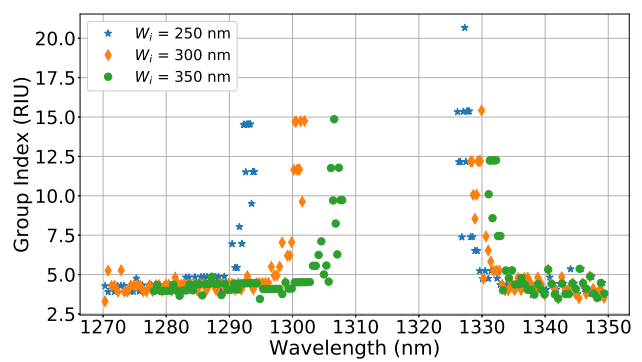

(a)

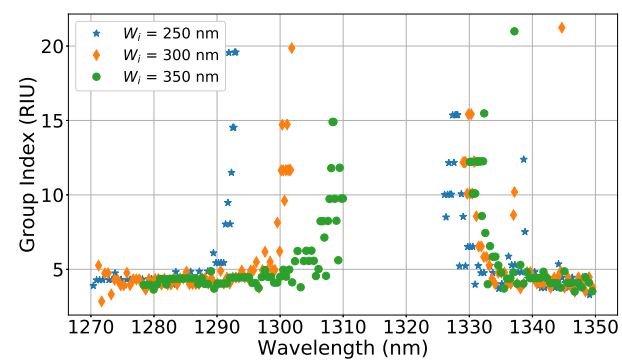

(b)

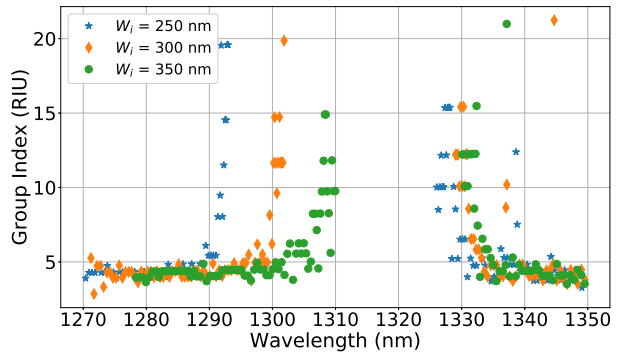

(c)

Figure 3.21: Group index distribution of the measured $\mathrm{PhC}$ with double apodisation: (a) Blackman, (b) Raised Sine and (c) Linear. Three different geometries have been measured with $W_{i}=$ 250,300 and $350 \mathrm{~nm}$.

higher dispersion, causing light to slow down even more when compared to a single taper design. Once again, it is important to notice that the homogeneity of the results across the nine different designs.

However, as pointed out previously, due to setup limitation we have not been able to properly characterize $n_{g}$ for the $3^{\text {rd }}$ band. Fig. 3.21 show that it was not possible to measure, any increase of the group index below $1285 \mathrm{~nm}$, for any of the different combination. Therefore, a more extensive analysis below $1290 \mathrm{~nm}$ should be performed in order to fully characterize the $3^{\text {rd }}$ band of the $1 \mathrm{D}-\mathrm{PhC}$.

In addition, it is of great importance to also evaluate the efficiency (FOM ${ }^{4}$ ) of each band and each design. Using the same method described in Sec. 3.3.2, we obtained interesting results.

\footnotetext{
${ }^{4} \mathrm{As}$ in Sec. 3.3 .2 the propagation losses values have been normalized to $1 \mathrm{~mm}$ for clearance.
} 


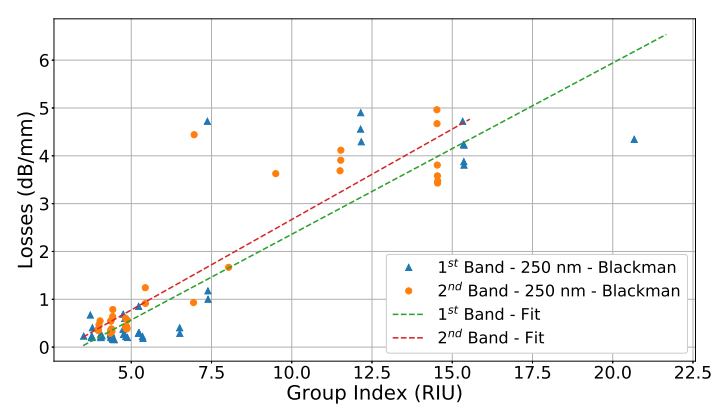

(a)

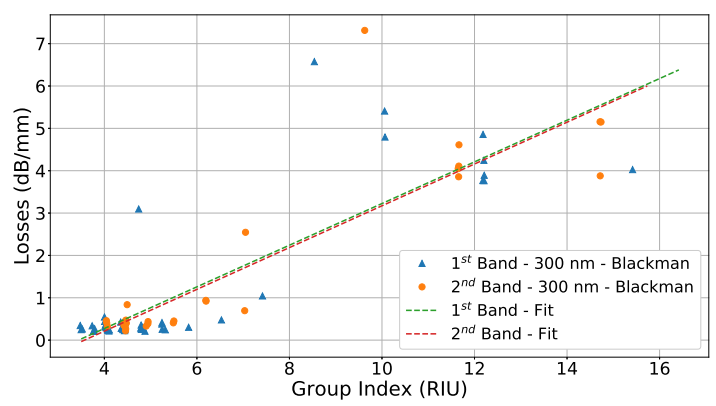

(b)

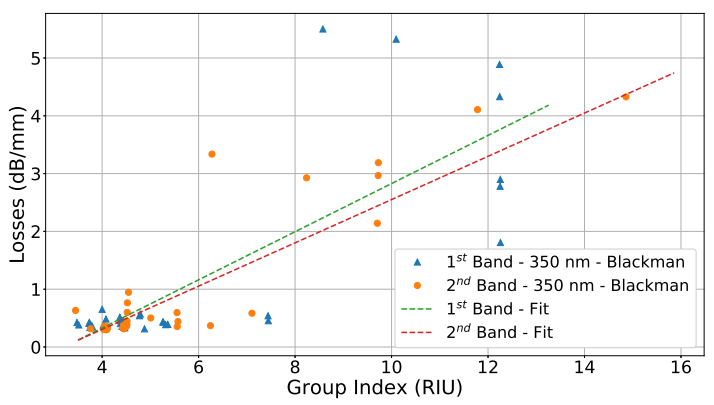

(c)

Figure 3.22: FOM values for both bands of the Blackman double apodization geometry: (a) $W_{i}$ $=250 \mathrm{~nm}$, (b) $W_{i}=300 \mathrm{~nm}$ and (c) $=350 \mathrm{~nm}$.

\begin{tabular}{c|c|c|c}
\hline & $\begin{array}{c}250 \mathrm{~nm} \\
(\mathrm{~dB} / \mathrm{RIU})\end{array}$ & $\begin{array}{c}300 \mathrm{~nm} \\
(\mathrm{~dB} / \mathrm{RIU})\end{array}$ & $\begin{array}{c}350 \mathrm{~nm} \\
(\mathrm{~dB} / \mathrm{RIU})\end{array}$ \\
\hline $1^{\text {st }}$ Band & 0.36 & 0.49 & 0.42 \\
$2^{\text {nd }}$ Band & 0.38 & 0.49 & 0.37 \\
\hline
\end{tabular}

Table 3.6: FOM values for the Blackman double apodisation geometries $\left(W_{i}=250,300,350\right.$ $\mathrm{nm})$ for the $1^{\text {st }}$ and $2^{\text {nd }}$ band.

Starting from the Blackman apodisation (Fig. 3.22 and Table 3.6). It is immediately clear that the two bands, thanks to the implementation of a double apodisation, have the same performances in terms of $\mathrm{dB} / \mathrm{RIU}$. Consequently, a nearly symmetrical PBG is created both in terms of spectra, as seen before in Fig. 3.19, and efficiency. The three different values of $W_{i}$ shows a better efficiency in the 250 and $350 \mathrm{~nm}$ cases, with the 300 case being a little less efficient. However, these values are quite small when compared to the normal length of a 1D-PhC. 


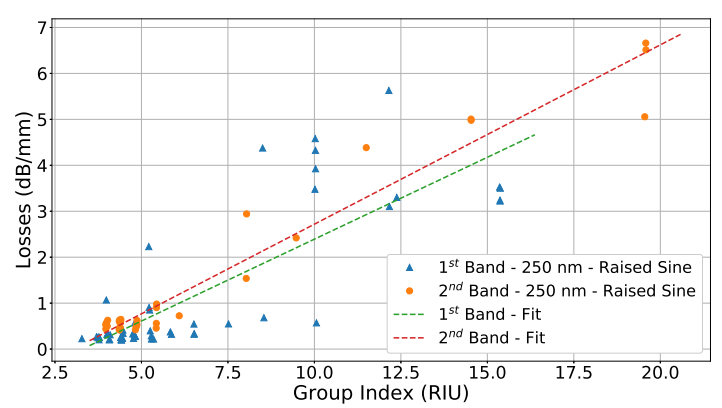

(a)

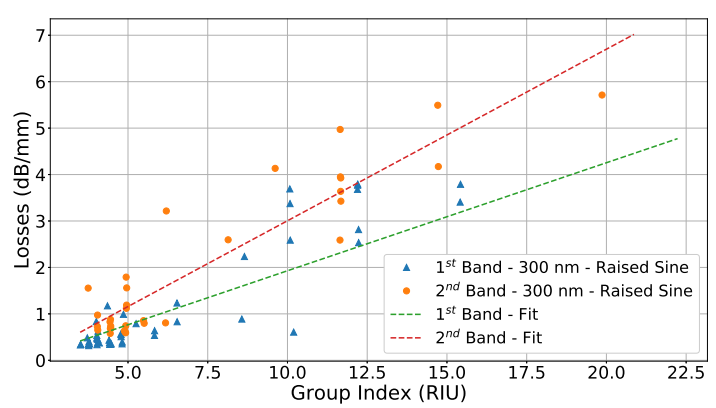

(b)

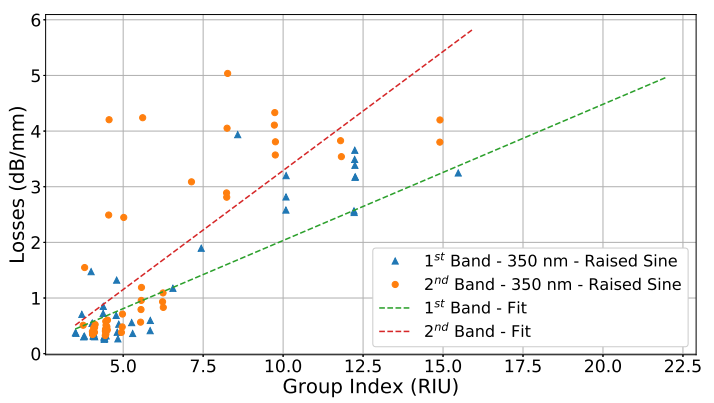

(c)

Figure 3.23: FOM values for both bands of the Raised Sine double apodization geometry: (a) $W_{i}=250 \mathrm{~nm}$, (b) $W_{i}=300 \mathrm{~nm}$ and (c) $=350 \mathrm{~nm}$.

\begin{tabular}{c|c|c|c}
\hline & $\begin{array}{c}250 \mathrm{~nm} \\
(\mathrm{~dB} / \mathrm{RIU})\end{array}$ & $\begin{array}{c}300 \mathrm{~nm} \\
(\mathrm{~dB} / \mathrm{RIU})\end{array}$ & $\begin{array}{c}350 \mathrm{~nm} \\
(\mathrm{~dB} / \mathrm{RIU})\end{array}$ \\
\hline $1^{\text {st }}$ Band & 0.35 & 0.23 & 0.24 \\
$2^{\text {nd }}$ Band & 0.39 & 0.37 & 0.42 \\
\hline
\end{tabular}

Table 3.7: FOM values for the Raised Sine double apodisation geometries $\left(W_{i}=250,300,350\right.$ $\mathrm{nm})$ for the $1^{\text {st }}$ and $2^{\text {nd }}$ band.

The Raised Sine apodization (Fig. 3.23 and Table 3.7) affects the results in a different way. The case for $W_{i}$ equal 300 and $350 \mathrm{~nm}$, does not show a symmetric performance for of the PBG. Instead, their behaviour resembles the trend presented in Sec. 3.3 .2 and shown in Fig. 3.12 for the single taper apodisation, while the first band more efficient than the second band, in terms of losses for increase of $n_{g}$. However, a noticeable improvement in terms of similar efficiency can be perceived when observing the $250 \mathrm{~nm}$ case, at the expense of first band efficiency.

The Linear apodization (Fig. 3.24 and Table 3.8) results are really similar to the 


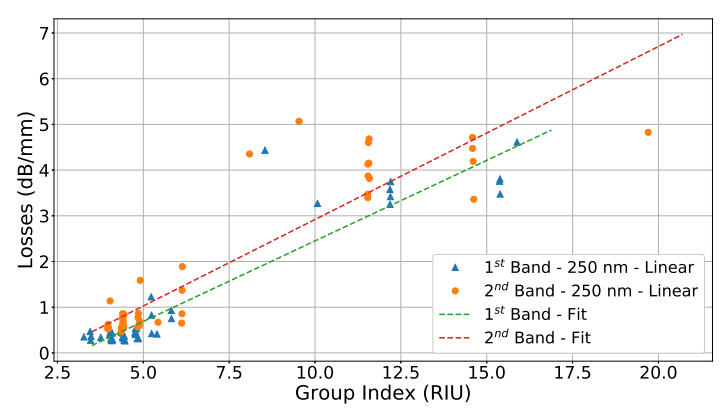

(a)

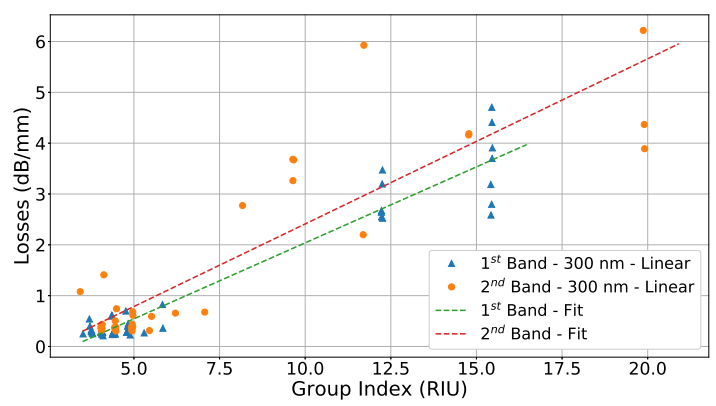

(b)

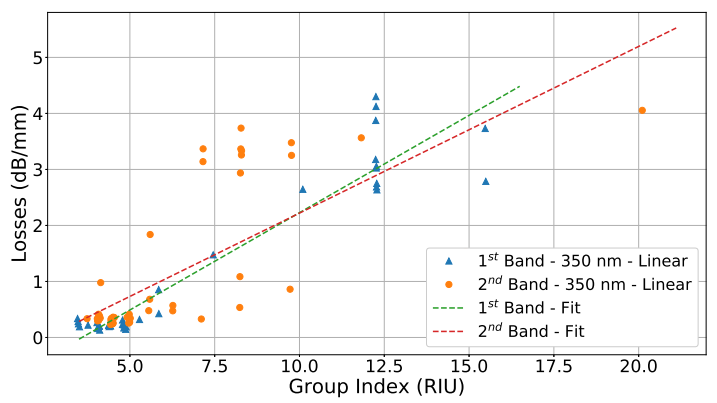

(c)

Figure 3.24: FOM values for both bands of the Linear double apodization geometry: (a) $W_{i}=$ $250 \mathrm{~nm},(\mathrm{~b}) W_{i}=300 \mathrm{~nm}$ and $(\mathrm{c})=350 \mathrm{~nm}$.

\begin{tabular}{c|c|c|c}
\hline & $\begin{array}{c}250 \mathrm{~nm} \\
(\mathrm{~dB} / \mathrm{RIU})\end{array}$ & $\begin{array}{c}300 \mathrm{~nm} \\
(\mathrm{~dB} / \mathrm{RIU})\end{array}$ & $\begin{array}{c}350 \mathrm{~nm} \\
(\mathrm{~dB} / \mathrm{RIU})\end{array}$ \\
\hline $1^{\text {st }}$ Band & 0.35 & 0.29 & 0.34 \\
$2^{\text {nd }}$ Band & 0.38 & 0.32 & 0.29 \\
\hline
\end{tabular}

Table 3.8: FOM values for the Linear double apodisation geometries $\left(W_{i}=250,300,350 \mathrm{~nm}\right)$ for the $1^{\text {st }}$ and $2^{\text {nd }}$ band.

BKM case. The three different designs show great homogeneity, allowing for the creation of symmetric PBG. Observing closely the FOM values, it is possible to see that for $W_{i}=300$ and $350 \mathrm{~nm}$, the FOM is slightly lower than the remaining case. The small penalty for $W_{i}=250 \mathrm{~nm}$ can still be considerate acceptable if the designed application requests the features given by this specific design.

In conclusion, the double apodization geometry permits to suppress the Fabry-Perot resonances of the $2^{\text {nd }}$ band edge, giving also higher levels of tunability when compared to the single apodization approach and other layouts [40]. The application of 
LN and BKM allowed the creation of a highly efficient symmetric PBG, with relative low propagation losses and high values of $n_{g}$. However, the RS has shown discrepancy in terms of efficiency, by allowing the two bands to have the same efficiency. This aspect has to be well understood and further analysis must be performed.

\subsection{Conclusions}

In conclusion, we presented the design, fabrication and characterization of two novel geometries of $1 \mathrm{D}-\mathrm{PhC}$ that minimize and solve two issues of standard 1D$\mathrm{PhC}$ : Fabry-Perot resonances and optical mismatch from the fast-light to slow-light regimes. Our experimental results shows that the novel designs are characterized by efficient trade-off between losses and $n_{g}$. Additionally, our study gives more insights on how the apodisation affects the different modes of the a $\mathrm{PhC}$, and how it might be possible to further increase the performance of such devices.

Overall, this type of slow-light structure might increase the readiness of the technology, and, as a consequence, also the number of application in which they are used, such as CWDM application for data centres and HPC, owing to their high tunability, high optical bandwidth and precise fabrication when comparing to other structures, such as AWG and 2D-PhC. 



\section{Bibliography}

[1] Martijn JR Heck and John E Bowers. Energy efficient and energy proportional optical interconnects for multi-core processors: Driving the need for on-chip sources. IEEE Journal of Selected Topics in Quantum Electronics, 20(4):332$343,2013$.

[2] Marianna Pantouvaki, Peter De Heyn, Michal Rakowski, Peter Verheyen, Brad Snyder, SA Srinivasan, Hongtao Chen, Jeroen De Coster, Guy Lepage, Philippe Absil, et al. $50 \mathrm{gb} / \mathrm{s}$ silicon photonics platform for short-reach optical interconnects. In Optical Fiber Communication Conference, pages Th4H-4. Optical Society of America, 2016.

[3] Runxiang Yu, Stanley Cheung, Yuliang Li, Katsunari Okamoto, Roberto Proietti, Yawei Yin, and SJB Yoo. A scalable silicon photonic chip-scale optical switch for high performance computing systems. Optics Express, 21(26):3265532667, 2013.

[4] Ting Yang, Jianji Dong, Li Liu, Shasha Liao, Sisi Tan, Lei Shi, Dingshan Gao, and Xinliang Zhang. Experimental observation of optical differentiation and optical hilbert transformation using a single soi microdisk chip. Scientific reports, 4(1):1-6, 2014.

[5] José Juan Colás. The electro-photonic silicon biosensor. In Dual-Mode Electro-photonic Silicon Biosensors, pages 59-93. Springer, 2017.

[6] Siming Chen, Wei Li, Jiang Wu, Qi Jiang, Mingchu Tang, Samuel Shutts, Stella N Elliott, Angela Sobiesierski, Alwyn J Seeds, Ian Ross, et al. Electrically pumped continuous-wave iii-v quantum dot lasers on silicon. Nature Photonics, 10(5):307, 2016.

[7] David J Thomson, Frederic Y Gardes, Jean-Marc Fedeli, Sanja Zlatanovic, Youfang Hu, Bill Ping Piu Kuo, Evgeny Myslivets, Nikola Alic, Stojan Radic, Goran Z Mashanovich, et al. 50-gb/s silicon optical modulator. IEEE Photonics Technology Letters, 24(4):234-236, 2011.

[8] FY Gardes, A Brimont, P Sanchis, G Rasigade, D Marris-Morini, L O'Faolain, F Dong, JM Fedeli, Pieter Dumon, L Vivien, et al. High-speed modulation of 
a compact silicon ring resonator based on a reverse-biased pn diode. Optics express, 17(24):21986-21991, 2009.

[9] Liangjun Lu, Shuoyi Zhao, Linjie Zhou, Dong Li, Zuxiang Li, Minjuan Wang, Xinwan Li, and Jianping Chen. 16× 16 non-blocking silicon optical switch based on electro-optic mach-zehnder interferometers. Optics express, 24(9):92959307, 2016.

[10] Keijiro Suzuki, Ken Tanizawa, Satoshi Suda, Hiroyuki Matsuura, Takashi Inoue, Kazuhiro Ikeda, Shu Namiki, and Hitoshi Kawashima. Broadband silicon photonics $8 \times 8$ switch based on double-mach-zehnder element switches. Optics express, 25(7):7538-7546, 2017.

[11] Andrea Zanzi, Christos Vagionas, Amadeu Griol, Alvaro Rosa, Sergio Lechago, Miltiadis Moralis-Pegios, Konstantinos Vyrsokinos, Nikos Pleros, Jochen Kraft, Victor Sidorov, et al. Alignment tolerant, low voltage, 0.23 v. cm, push-pull silicon photonic switches based on a vertical pn junction. Optics express, 27(22):32409-32426, 2019.

[12] Chen Sun, Mark T Wade, Yunsup Lee, Jason S Orcutt, Luca Alloatti, Michael S Georgas, Andrew S Waterman, Jeffrey M Shainline, Rimas R Avizienis, Sen Lin, et al. Single-chip microprocessor that communicates directly using light. Nature, 528(7583):534-538, 2015.

[13] Bahaa EA Saleh and Malvin Carl Teich. Fundamentals of photonics. john Wiley \& sons, 2019.

[14] Eli Yablonovitch. Inhibited spontaneous emission in solid-state physics and electronics. Physical review letters, 58(20):2059, 1987.

[15] Sajeev John. Strong localization of photons in certain disordered dielectric superlattices. Physical review letters, 58(23):2486, 1987.

[16] Susumu Noda, Masayuki Fujita, and Takashi Asano. Spontaneous-emission control by photonic crystals and nanocavities. Nature photonics, 1(8):449, 2007.

[17] MD Lukin. Colloquium: Trapping and manipulating photon states in atomic ensembles. Reviews of Modern Physics, 75(2):457, 2003. 
[18] Fumio Koyama. Recent advances of vcsel photonics. Journal of lightwave technology, 24(12):4502-4513, 2006.

[19] Chun-Feng Lai. Luminescence-spectrum modification of white light-emitting diodes by using 3d colloidal photonic crystals. Photonic Crystals, page 75, 2015.

[20] Ian B Burgess, Lidiya Mishchenko, Benjamin D Hatton, Mathias Kolle, Marko Loncar, and Joanna Aizenberg. Encoding complex wettability patterns in chemically functionalized $3 \mathrm{~d}$ photonic crystals. Journal of the American Chemical Society, 133(32):12430-12432, 2011.

[21] Shinpei Ogawa, Masahiro Imada, Susumu Yoshimoto, Makoto Okano, and Susumu Noda. Control of light emission by 3d photonic crystals. Science, 305(5681):227-229, 2004.

[22] Eric W Seelig, Betty Tang, Alexey Yamilov, Hui Cao, and Robert PH Chang. Self-assembled 3d photonic crystals from zno colloidal spheres. Materials Chemistry and Physics, 80(1):257-263, 2003.

[23] Przemek J Bock, Pavel Cheben, Jens H Schmid, Jean Lapointe, André Delâge, Dan-Xia Xu, Siegfried Janz, Adam Densmore, and Trevor J Hall. Subwavelength grating crossings for silicon wire waveguides. Optics express, 18(15):16146-16155, 2010.

[24] A Maese-Novo, R Halir, S Romero-García, D Pérez-Galacho, L ZavargoPeche, A Ortega-Moñux, I Molina-Fernández, JG Wangüemert-Pérez, and $\mathrm{P}$ Cheben. Wavelength independent multimode interference coupler. Optics express, 21(6):7033-7040, 2013.

[25] Robert Halir, Pavel Cheben, Siegfried Janz, Dan-Xia Xu, Íñigo MolinaFernández, and Juan G Wangüemert-Pérez. Waveguide grating coupler with subwavelength microstructures. Optics letters, 34(9):1408-1410, 2009.

[26] OV Ivanova, Remco Stoffer, Lasse Kauppinen, and Manfred Hammer. Variational effective index method for $3 \mathrm{~d}$ vectorial scattering problems in photonics: Te polarization. In Progress In Electromagnetics Research Symposium PIERS 2009, Moscow, Proceedings, pages 1038-1042, 2009. 
[27] Antoine Brimont, Jose Vicente Galán, Jose Maria Escalante, Javier Martí, and Pablo Sanchis. Group-index engineering in silicon corrugated waveguides. Optics letters, 35(16):2708-2710, 2010.

[28] Arman B Fallahkhair, Kai S Li, and Thomas E Murphy. Vector finite difference modesolver for anisotropic dielectric waveguides. Journal of Lightwave Technology, 26(11):1423-1431, 2008.

[29] Rsoft FullWave by SYNOPSYS. Photonic design software.

[30] RD Meade, Joshua N Winn, and JD Joannopoulos. Photonic crystals: Molding the flow of light. Princeton University Press Princeton, NJ, 1995.

[31] Raman Kashyap. Fiber bragg gratings. Academic press, 2009.

[32] Kazuaki Sakoda. Optical properties of photonic crystals, volume 80. Springer Science \& Business Media, 2004.

[33] Igor A Sukhoivanov and Igor V Guryev. Photonic crystals: physics and practical modeling, volume 152. Springer, 2009.

[34] S Lardenois, D Pascal, L Vivien, E Cassan, S Laval, R Orobtchouk, M Heitzmann, N Bouzaida, and L Mollard. Low-loss submicrometer silicon-on-insulator rib waveguides and corner mirrors. Optics letters, 28(13):1150-1152, 2003.

[35] Christelle Monat, Bill Corcoran, Majid Ebnali-Heidari, Christian Grillet, Benjamin J Eggleton, Thomas P White, Liam O'Faolain, and Thomas F Krauss. Slow light enhancement of nonlinear effects in silicon engineered photonic crystal waveguides. Optics express, 17(4):2944-2953, 2009.

[36] Hongyun Meng, Wei Shen, Guanbin Zhang, Chunhua Tan, and Xuguang Huang. Fiber bragg grating-based fiber sensor for simultaneous measurement of refractive index and temperature. Sensors and Actuators B: Chemical, 150(1):226-229, 2010.

[37] Victor Mizrahi and John E Sipe. Optical properties of photosensitive fiber phase gratings. Journal of lightwave technology, 11(10):1513-1517, 1993. 
[38] CR Giles. Lightwave applications of fiber bragg gratings. Journal of lightwave technology, 15(8):1391-1404, 1997.

[39] Tsuyoshi Horikawa, Daisuke Shimura, and Tohru Mogami. Low-loss silicon wire waveguides for optical integrated circuits. MRS Communications, 6(1):9-15, 2016.

[40] SA Schulz, Liam O'Faolain, Daryl M Beggs, TP White, A Melloni, and Thomas F Krauss. Dispersion engineered slow light in photonic crystals: a comparison. Journal of Optics, 12(10):104004, 2010. 



\section{Chapter 4}

\section{Conclusions and outlooks}

This work has provided advancement in silicon photonic devices demonstrating the design, fabrication and measurements of an A-MMI, a high speed silicon modulator and optimized geometries of 1D-PhCs.

In the first chapter, this work has demonstrated the feasibility of highly efficient and low power Mach-Zehnder silicon modulators featuring a broad optical bandwidth and state-of-the-art modulation efficiency. Nonetheless, in order to increase the level of readiness of these modulators, it is of paramount important to overcome the limitations arising from the traveling wave electrodes in order to ensure maximum $\mathrm{RF}$ performance and reach the full modulator intrinsic electro-optical bandwidth. A better understanding of AMS foundry's standard 2-layer BEOL metal stack allowed us (in collaboration with H2020-L3MATRIX project partner IZM) to address this electrical limitations from the design point of view and seamlessly implement the modifications in the final fabrication run that is currently under evaluation. The resulting optimized Mach-Zehnder modulator is expected to become a strong candidate for future high throughput transceivers in data centres and HPC applications. In the second chapter, this work showed novel multimode asymmetric interferences de-vices ready to be implemented in silicon photonic integrated circuits. Their small footprint, broad optical bandwidth and low-loss have allowed their implementation in asymmetric MZM to balance the optical power in each arm and achieve a higher extinction ratio compared to the symmetric version. In the third and final chapter, the designs of three novel apodisation schemes in $1 \mathrm{D}-\mathrm{PhCs}$ have been demonstrated, followed by an extensive analysis of their performance and trade-off 
between propagation losses and group index near the photonic band-gap. These slow light structures have been implemented in the final fabrication run of H2020L3MATRIX project and are currently being evaluated in highly efficient slow-light O-band modulators. The potential applications of these slow-light structures are indeed not limited to modulation applications but to a whole range of applications where high sensitivity (sensors) and or/low power operation (LIDARs, satellites, etc.) are required.

Overall, this thesis provides new insights in silicon-based technology and as the author, I firmly each chapter can be a starting point for further development of novel devices, both passive and active in silicon-on-insulator technology. In addition to its scientific and academic contributions, this work has a strong orientation towards industrial applications for the future and growth of photonic integrated circuits and their applications. Being able to improve and to always provide new and exciting contributions to the world is of vital importance. It is the dream of each scientist to create something new and contribute, even though a little bit, to the progress in his field of research and beyond. I hope that this thesis will help adding up more knowledge for the benefit of the scientific community and by extension for the progression of the humankind towards a bright future. 
Appendices 



\section{Appendix A}

\section{D-PhC Graph Appendix}

This appendix contains additional graphs regarding Sec. 3.3.3.

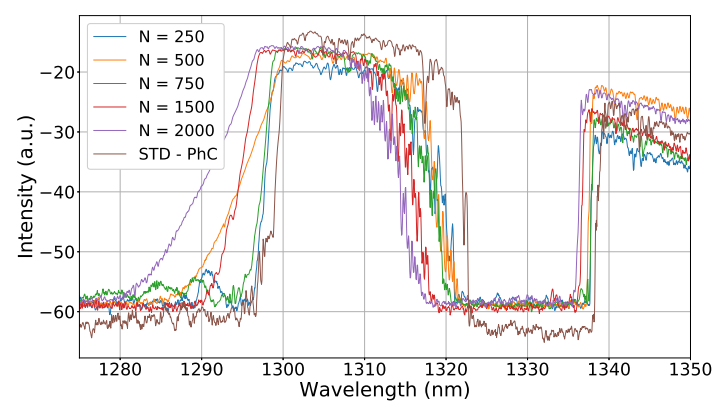

(a)

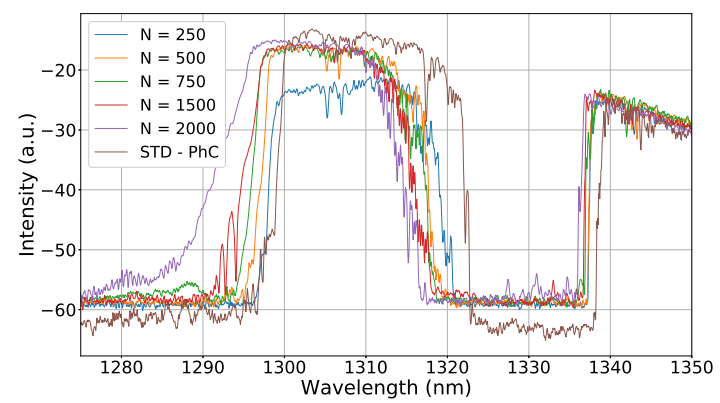

(b)

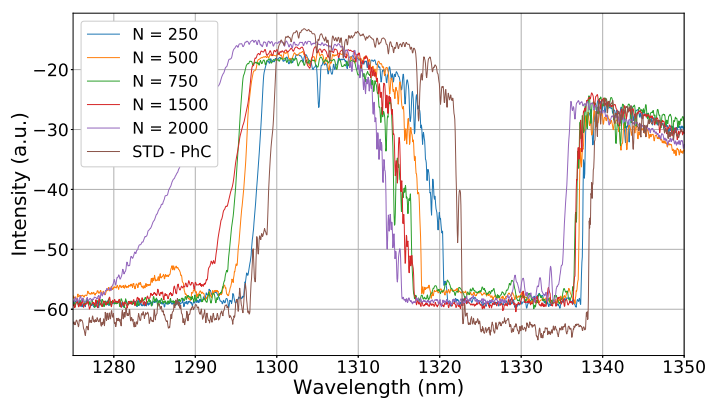

(c)

Figure A.1: Transmission spectra of the measured 1D-PhC with partial tapers: (a) Blackman, (b) Raised Sine and (c) Linear. Two different length were measured: 750 and 1500. The full tapered PhC (2000) has been added for comparison. 


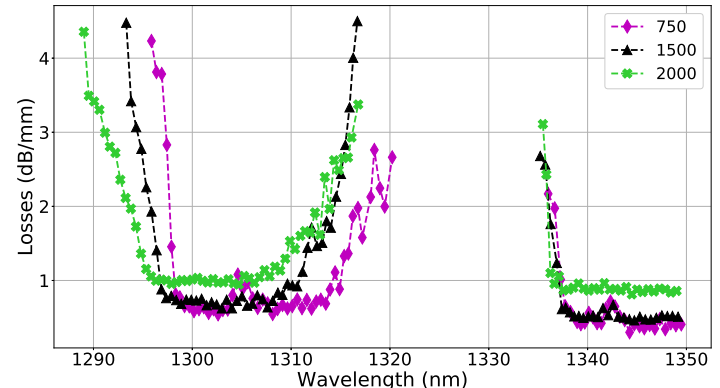

(a)

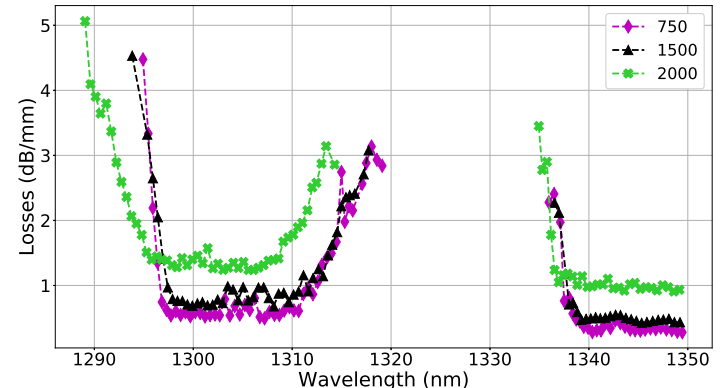

(b)

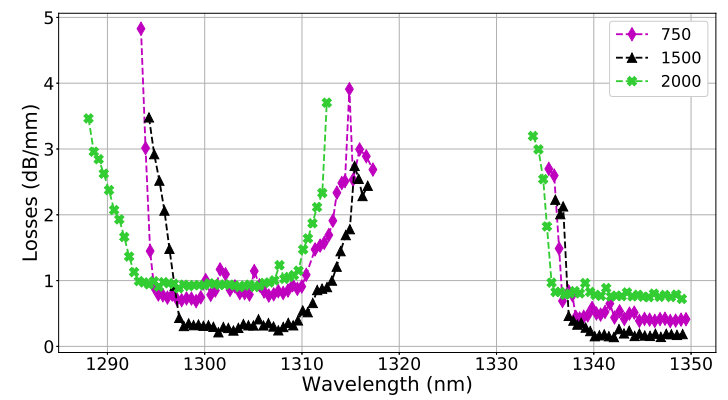

(c)

Figure A.2: Propagation losses of the measured 1D-PhC with partial tapers: (a) Blackman, (b) Raised Sine and (c) Linear. Two different length were measured: 750 and 1500. The full tapered $\mathrm{PhC}(2000)$ has been added for comparison. 


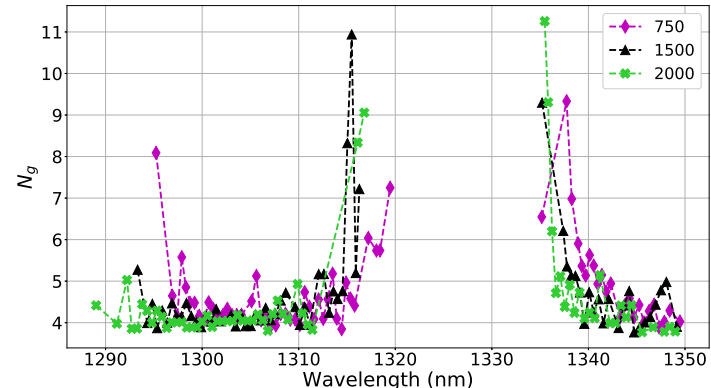

(a)

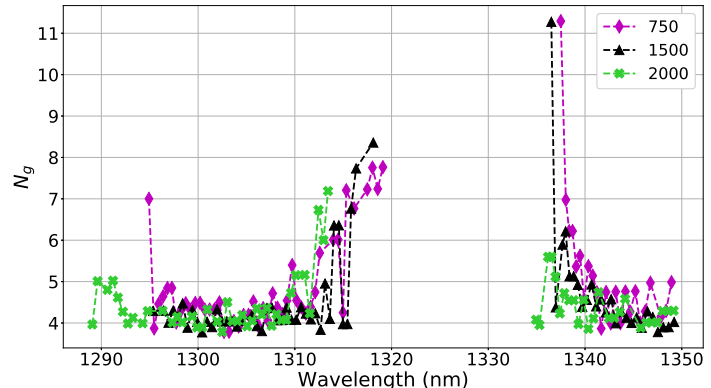

(b)

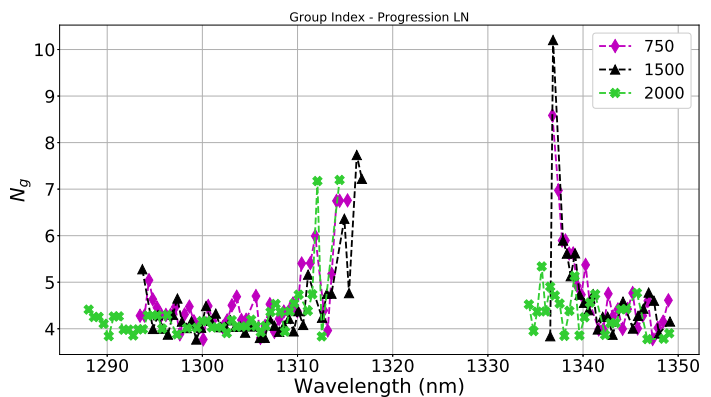

(c)

Figure A.3: Group index $\left(n_{g}\right)$ of the measured 1D-PhC with partial tapers: (a) Blackman, (b) Raised Sine and (c) Linear. Two different length were measured: 750 and 1500. The full tapered $\mathrm{PhC}(2000)$ has been added for comparison. 



\section{Appendix B}

\section{Silvaco code}

The following code simulates the fabrication process of a carrier depletion phaseshifter (ATHENA) and its static and transient behaviour (ATLAS) in a $400 \mathrm{~nm}$ silicon waveguide.

\section{B.1 ATHENA \& ATLAS simulation code}

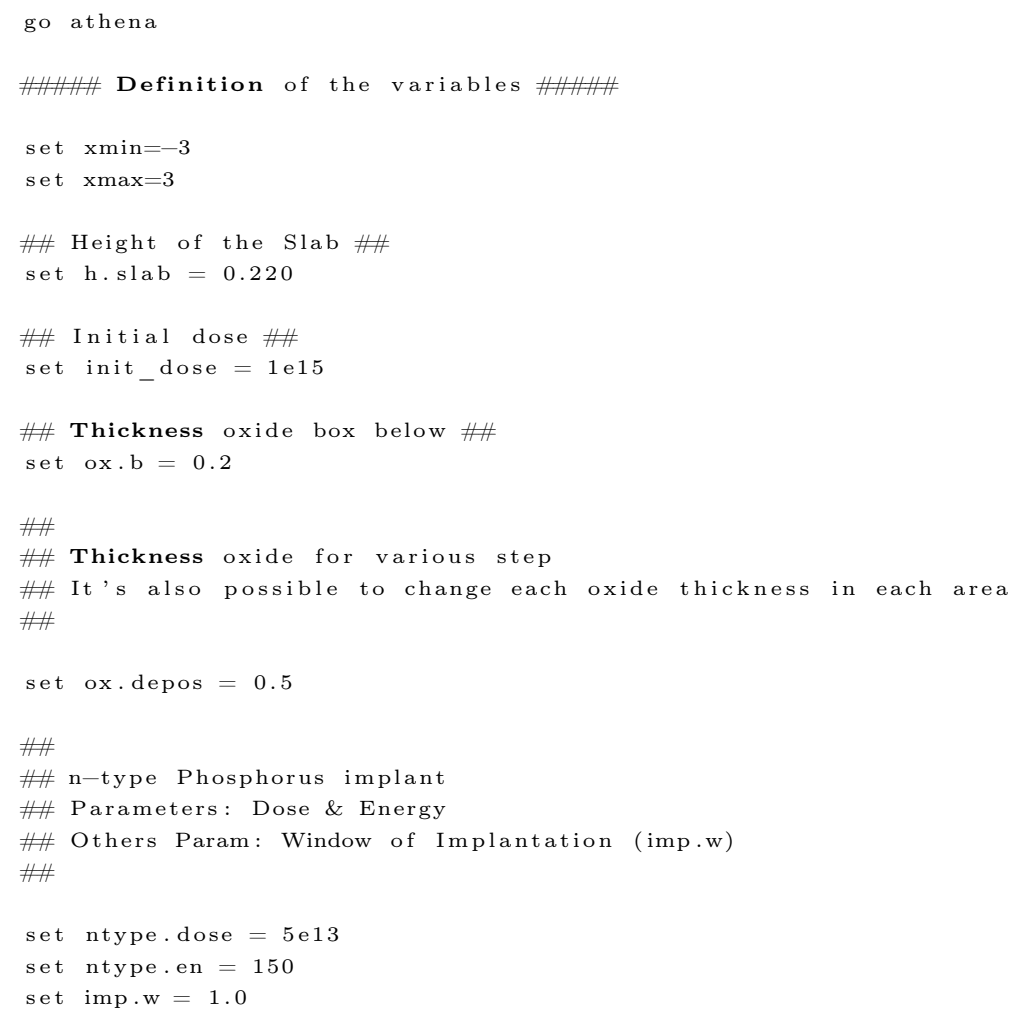




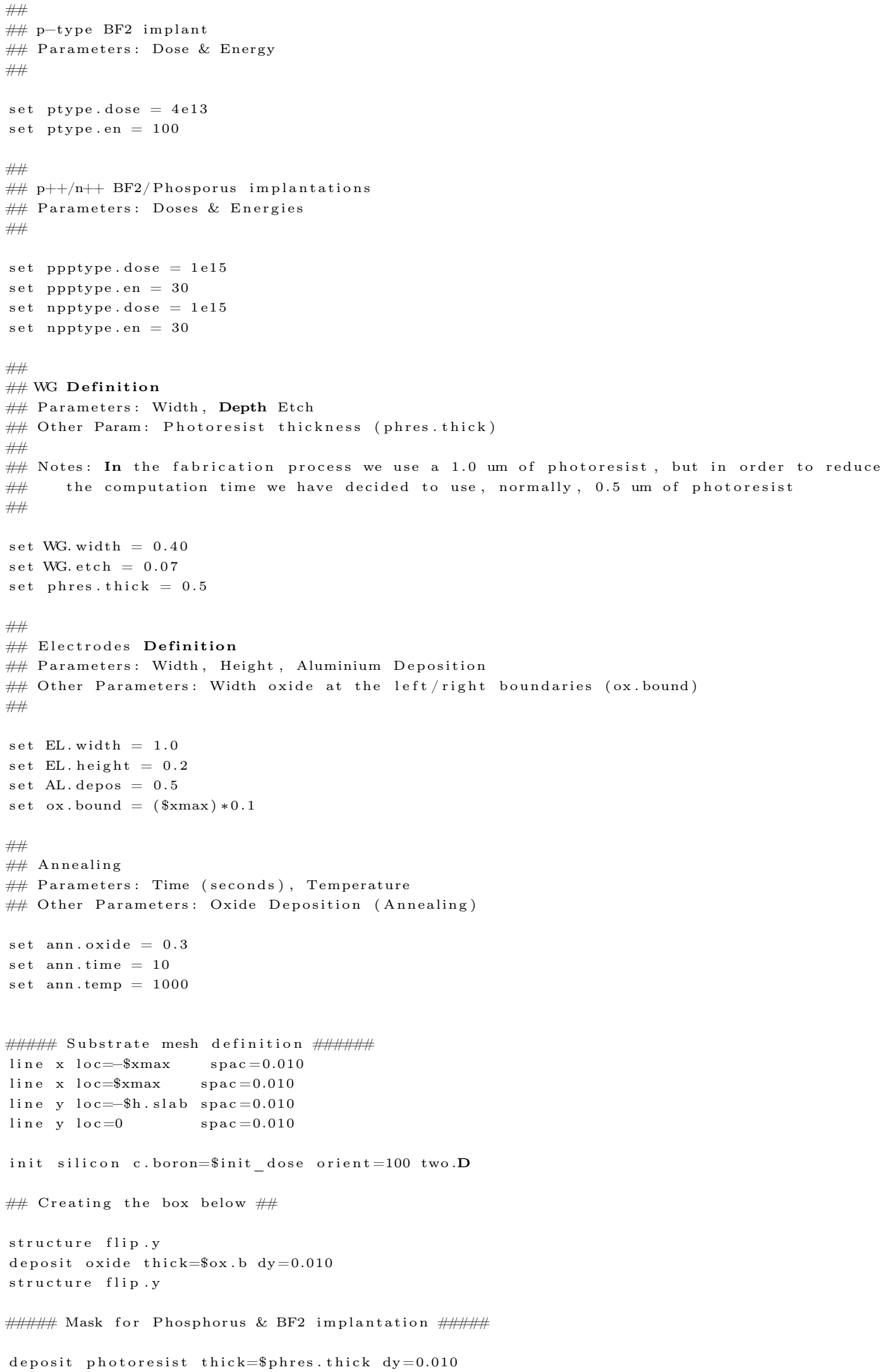




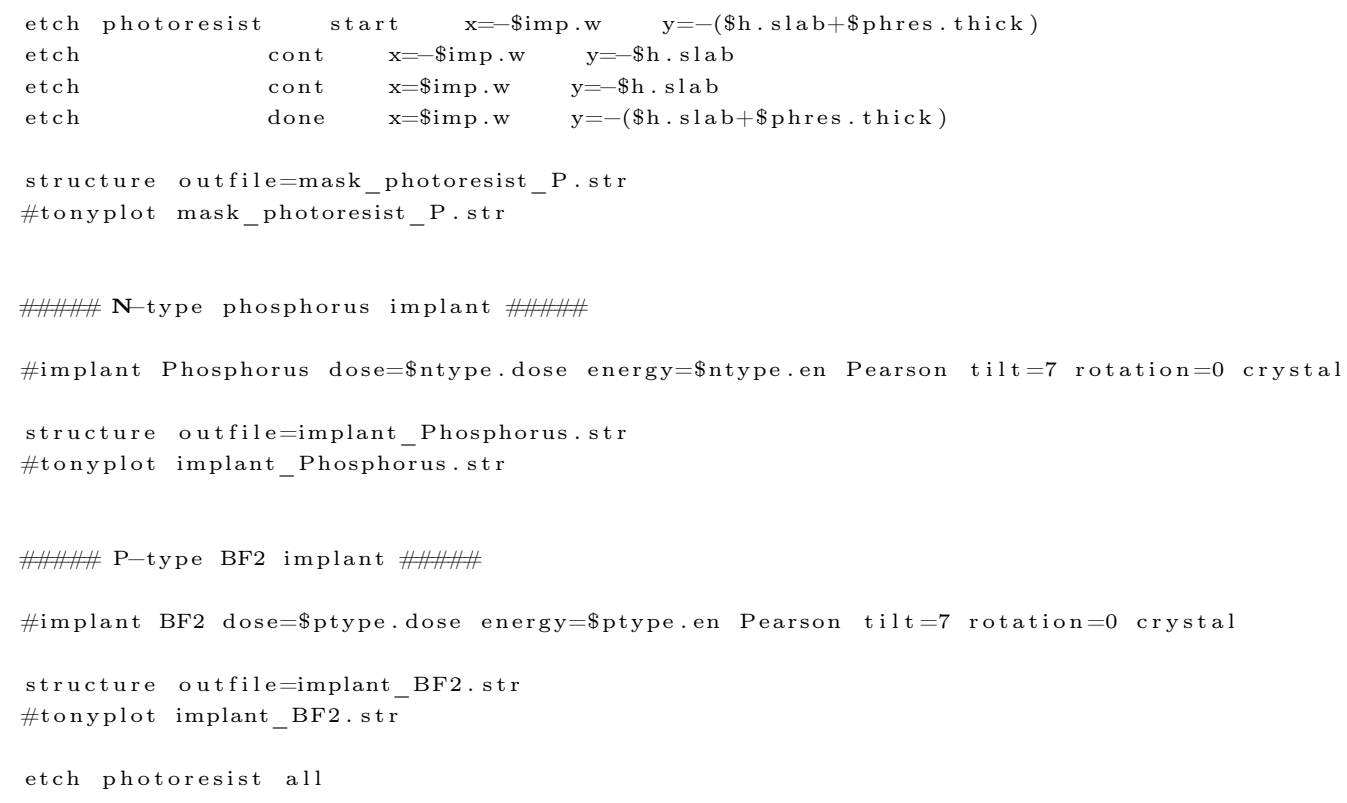

structure outfile=mask_WG.str \#tonyplot mask_WG.str

etch photoresist all 


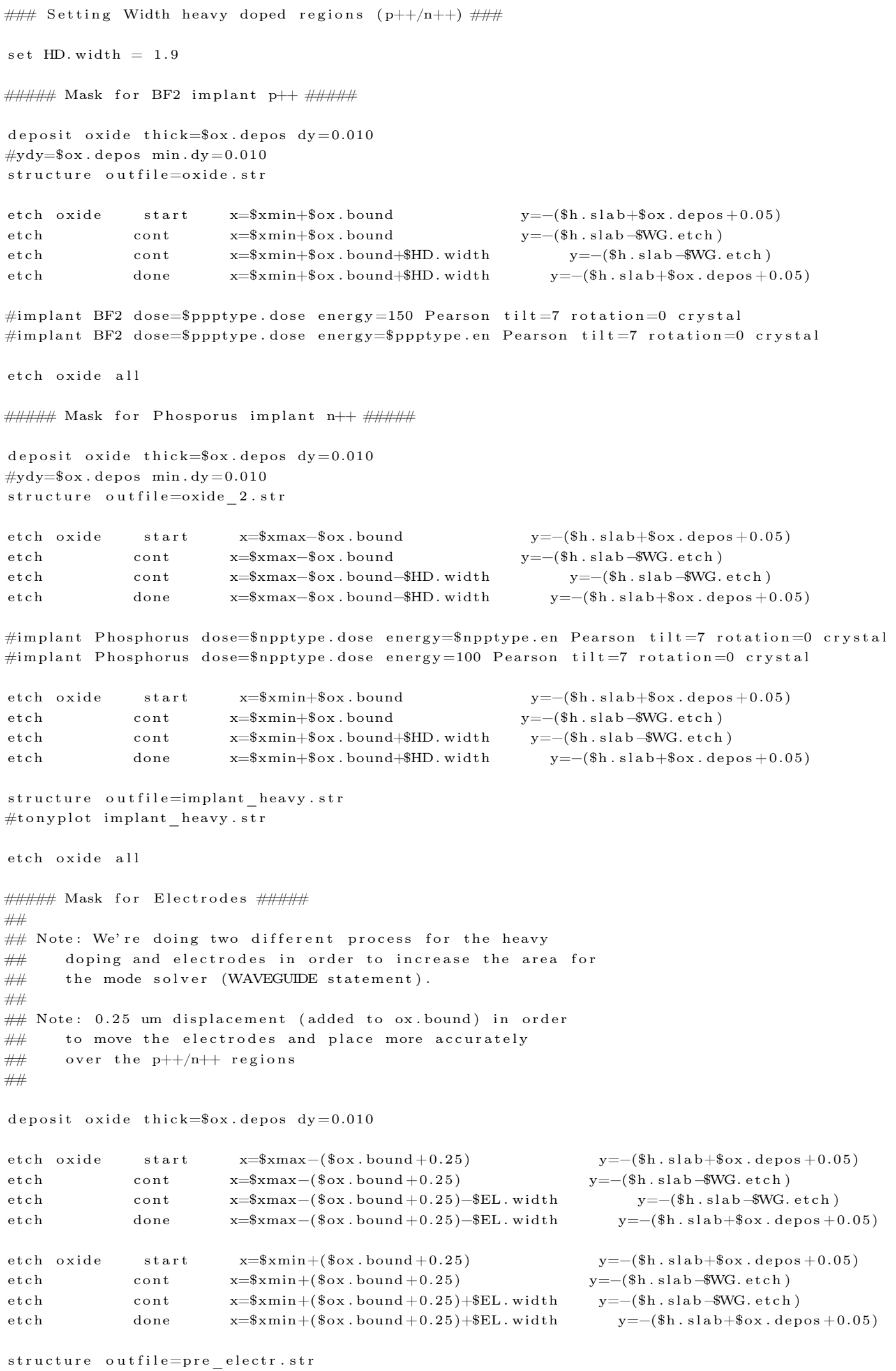




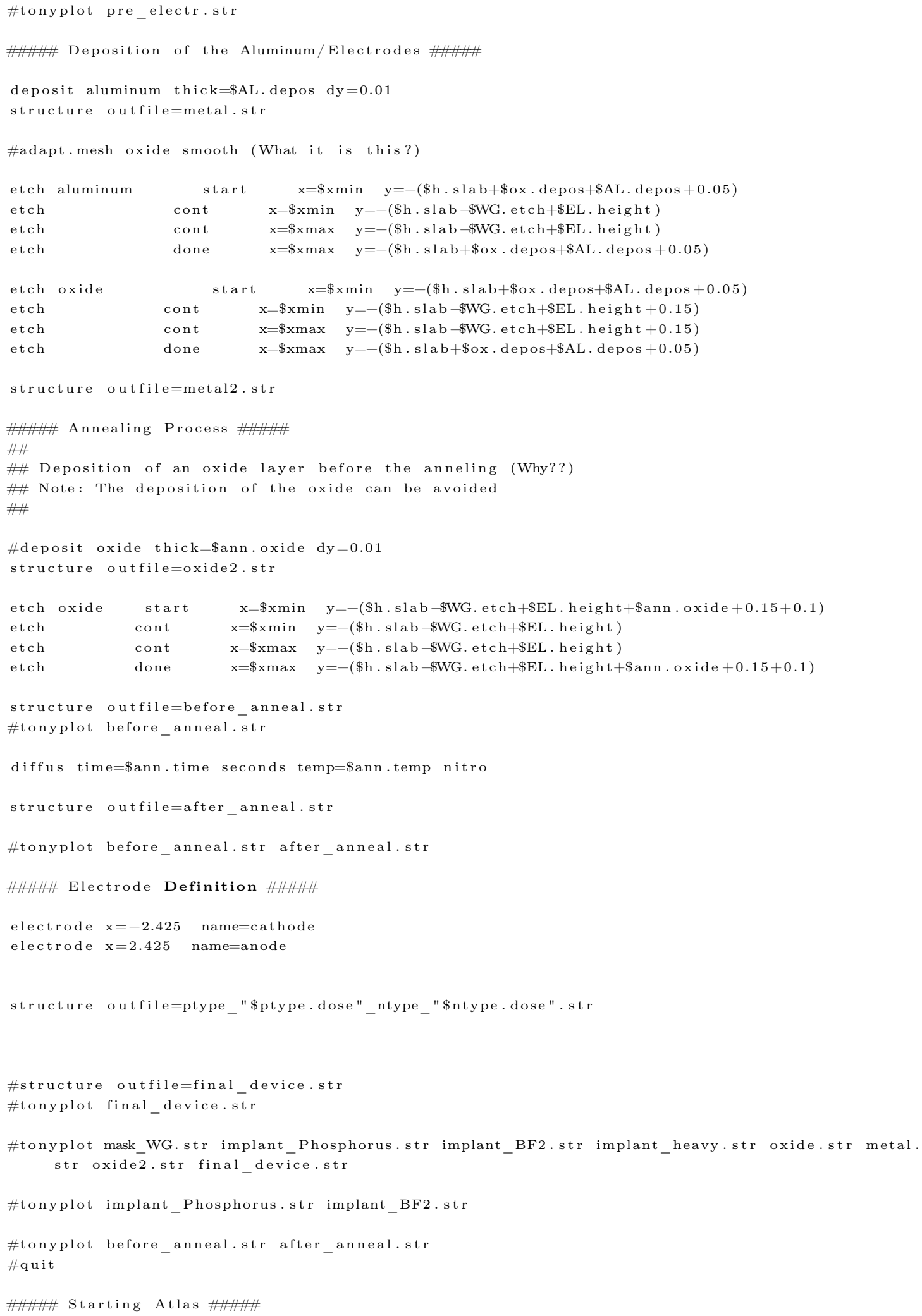




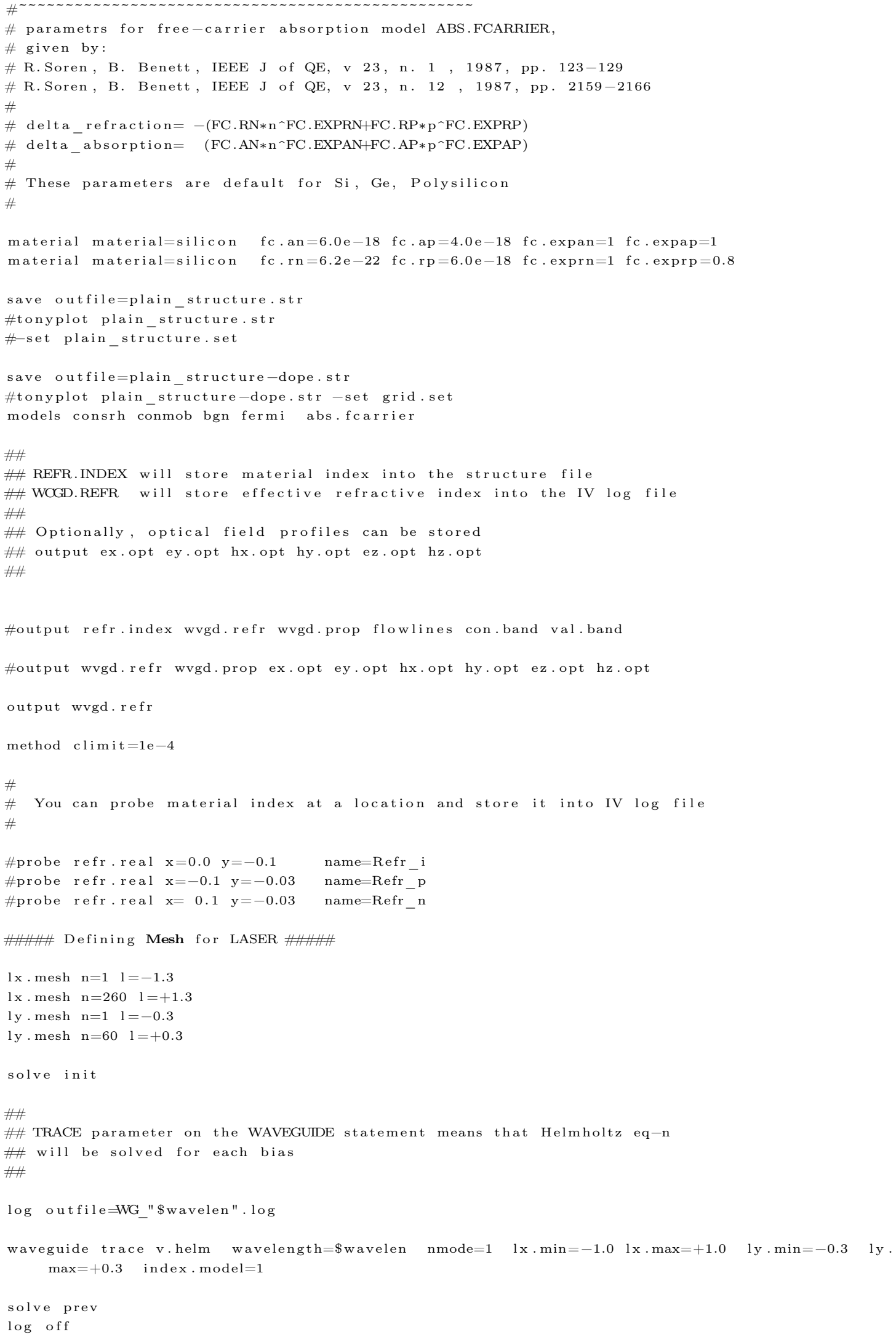




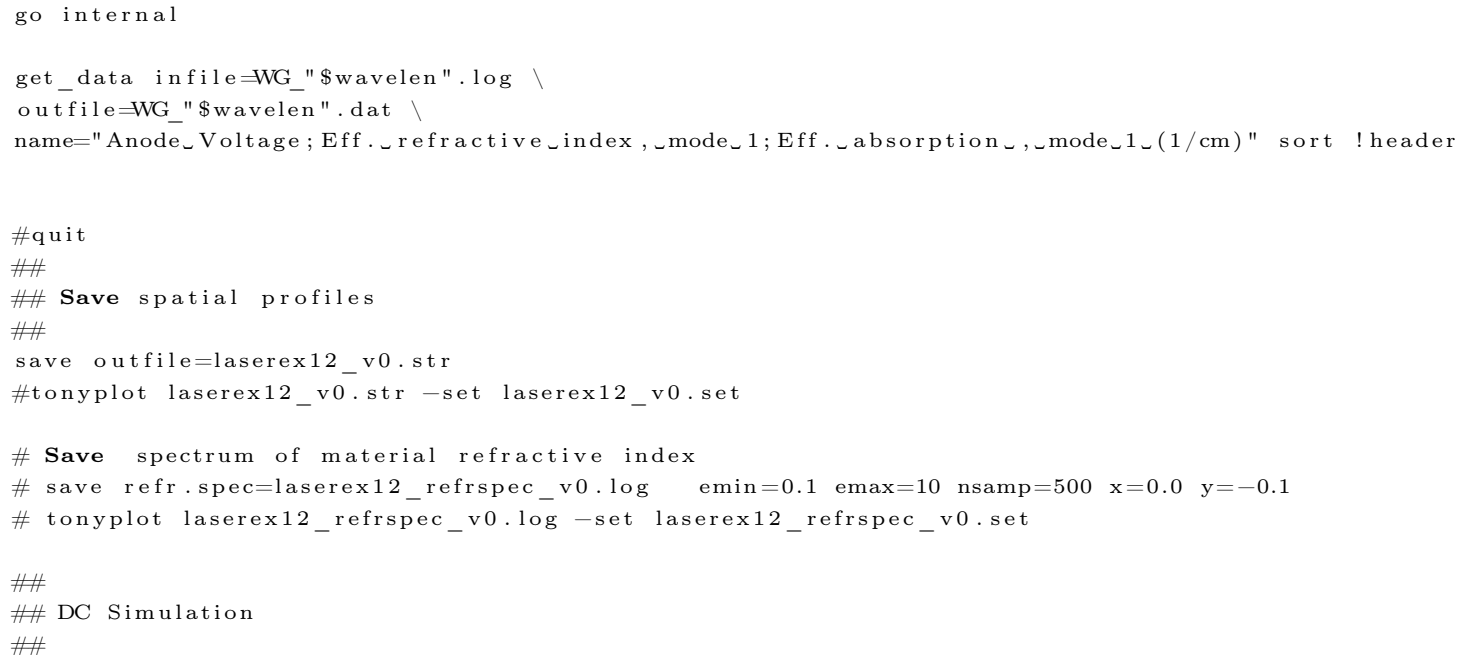


\#\#\#\# Exporting the data \#\#\#\#

go internal

get_data infile=AMS/ptype_"\$ptype.dose"_ntype_"\$ntype.dose".log

outfile=AMS/ptype_"\$ptype.dose"_ntype_" $\$$ ntype.dose".dat

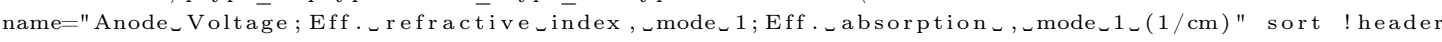

quit 


\section{Appendix C}

\section{FDTD}

This code is a TEz 2D-FDTD [1, 2] (Finite Difference Time Domain) written in Python. It has been used for the design of the 1D-PhCs presented in Chapter 3.

\section{C.1 2D-FDTD Code}

Listing C.1: Code of the TEz 2D-FDTD

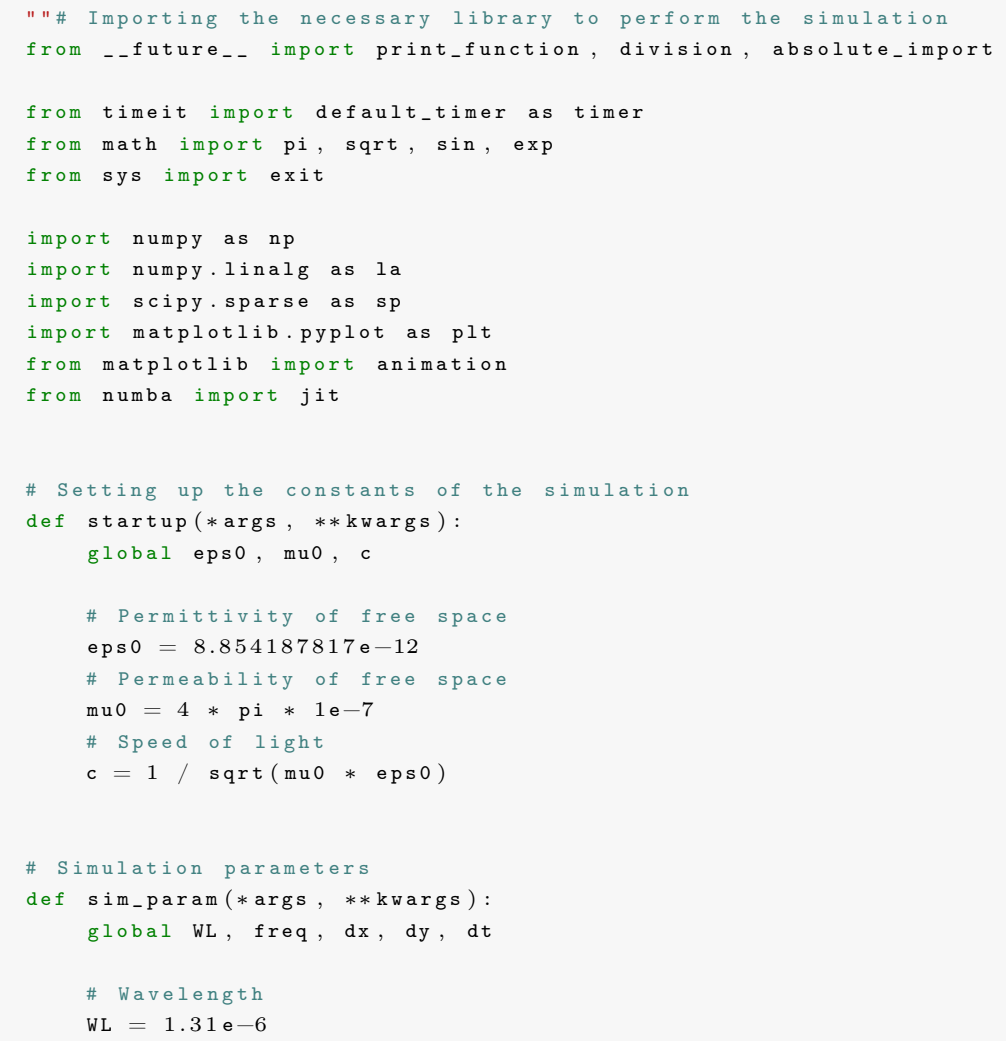




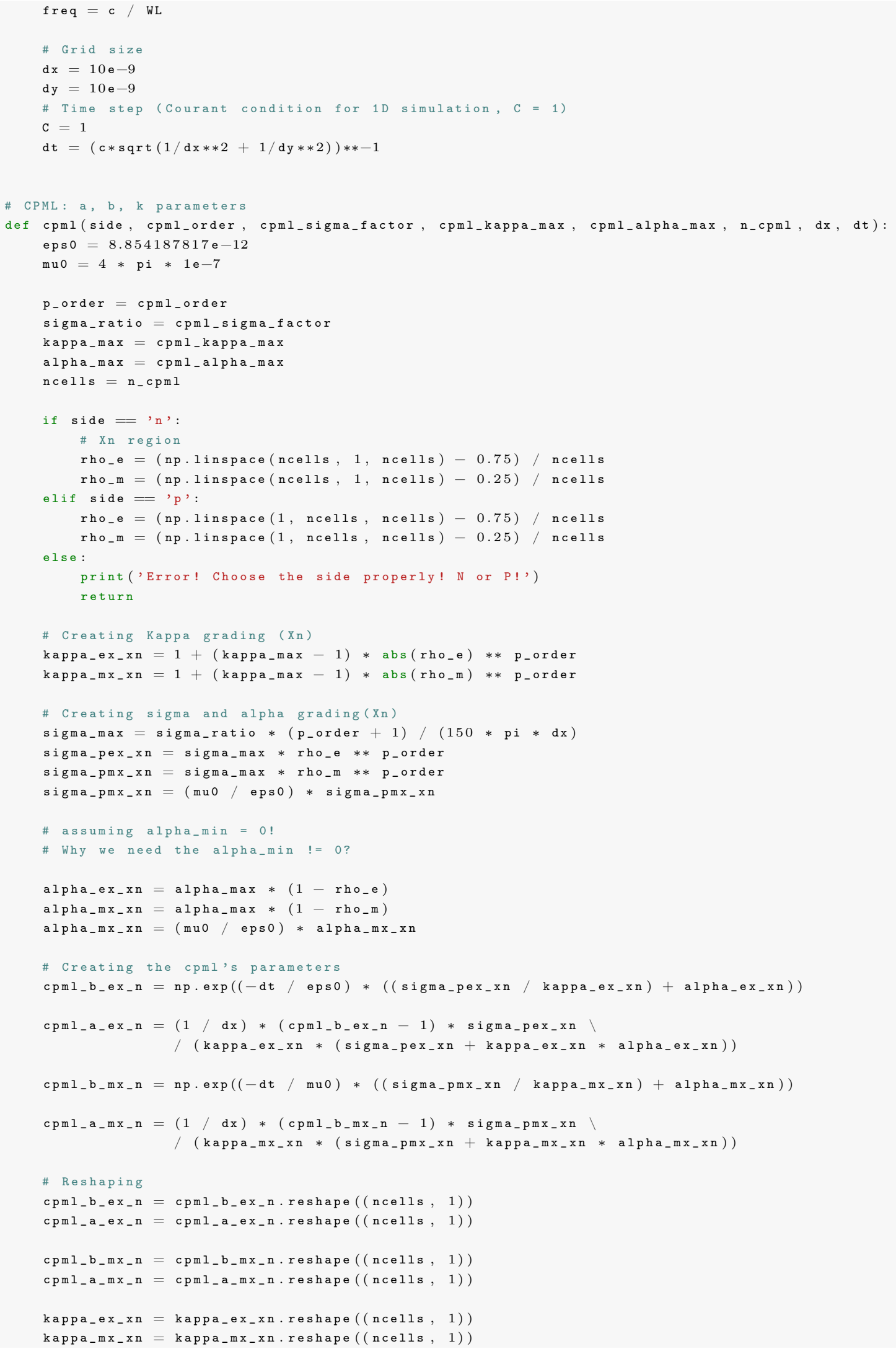


return cpml $b_{-} e x_{-} n, \quad c p m l_{-} a_{-} e x_{-} n, \quad c p m l_{-} b_{-} m x_{-} n, \quad c p m l_{-} a_{-} m x_{-} n, \quad k a p p a_{-} e x_{-} x_{n}, \quad k a p p a_{-} m x_{-} x_{n}$

\# Creating the Psi fields for the cpml, and updating the fields

def psi_cpml(*args, $* *$ kwargs ):

global cpml-b $b_{-} x_{-} x_{n}, c p m l_{-} a_{-} e x_{-} x_{n}, c p m l_{-} b_{-} m x_{-} x n, c p m l_{-} a_{-} m x_{-} x n, ।$ $c p m l_{-} b_{-} e x_{-} x p, \quad c p m l_{-} a_{-} e x_{-} x p, c p m l_{-} b_{-} m x_{-} x p, c p m l_{-} a_{-} m x_{-} x p$

global cpml_b_ey-yn, cpml_a_ey-yn, cpml_b-my-yn, cpml_a - $_{-} y_{-} y n$, $c p m l_{-} b_{-} e_{-} y_{p}, \quad c p m l_{-} a_{-} e_{-} y p, \quad c p m l_{-} b_{-} m_{-} y p, \quad c p m l_{-} a_{-} m y_{-} y p$

global Psi_hzx_xn, Psi_hzx-xp, Psi_hzy-yn, Psi_hzy-yp,

Psi_eyx-xn, Psi_eyx_xp, Psi_exy-yn, Psi_exy-yp

global CPsi_hzx_xn, CPsi_hzx_xp, CPsi_hzy-yn, CPsi_hzy-yp,

CPsi_eyx-xn, CPsi_eyx-xp, CPsi_exy-yn, CPsi_exy-yp

global $n_{-} c p m l$

\# CPML Parameters

$\mathrm{cpml}$ _order $=3$

$\mathrm{cpml}_{-} \mathrm{sigma}_{-}$factor $=1.3$

cpml_kappa_max $=7$

$c_{p m l_{-}} a_{p h a_{-} m a x}=0.05$

$\mathrm{n}_{-} \mathrm{cpml}=10$

\# X ( global)

$\left[c p m l_{-} b_{-} e x_{-} x n, \quad c p m l_{-} a_{-} e x_{-} x n, c p m l_{-} b_{-} m x_{-} x n, c p m l_{-} a_{-} m x_{-} x n, \quad k a p p a a_{-} e x_{-} x_{n}, \quad k a p p a a_{-} m x_{-} x n\right]=\backslash$ cpml ('n', cpml_order, cpml_sigma_factor, cpml_kappa_max, cpml_alpha_max, n_cpml, dx, dt)

$\left[c p m l_{-} b_{-} e x_{-} x p, c p m l_{-} a_{-} e x_{-} x p, c p m l_{-} b_{-} m x_{-} x p, c p m l_{-} a_{-} m x_{-} x p, \quad k a p p a_{-} e x_{-} x p, \quad k a p p a_{-} m x_{-} x p\right]=\backslash$ cpml ('p', cpml_order, cpml_sigma_factor, cpml_kappa_max, cpml_alpha_max, n_cpml, dx, dt) 


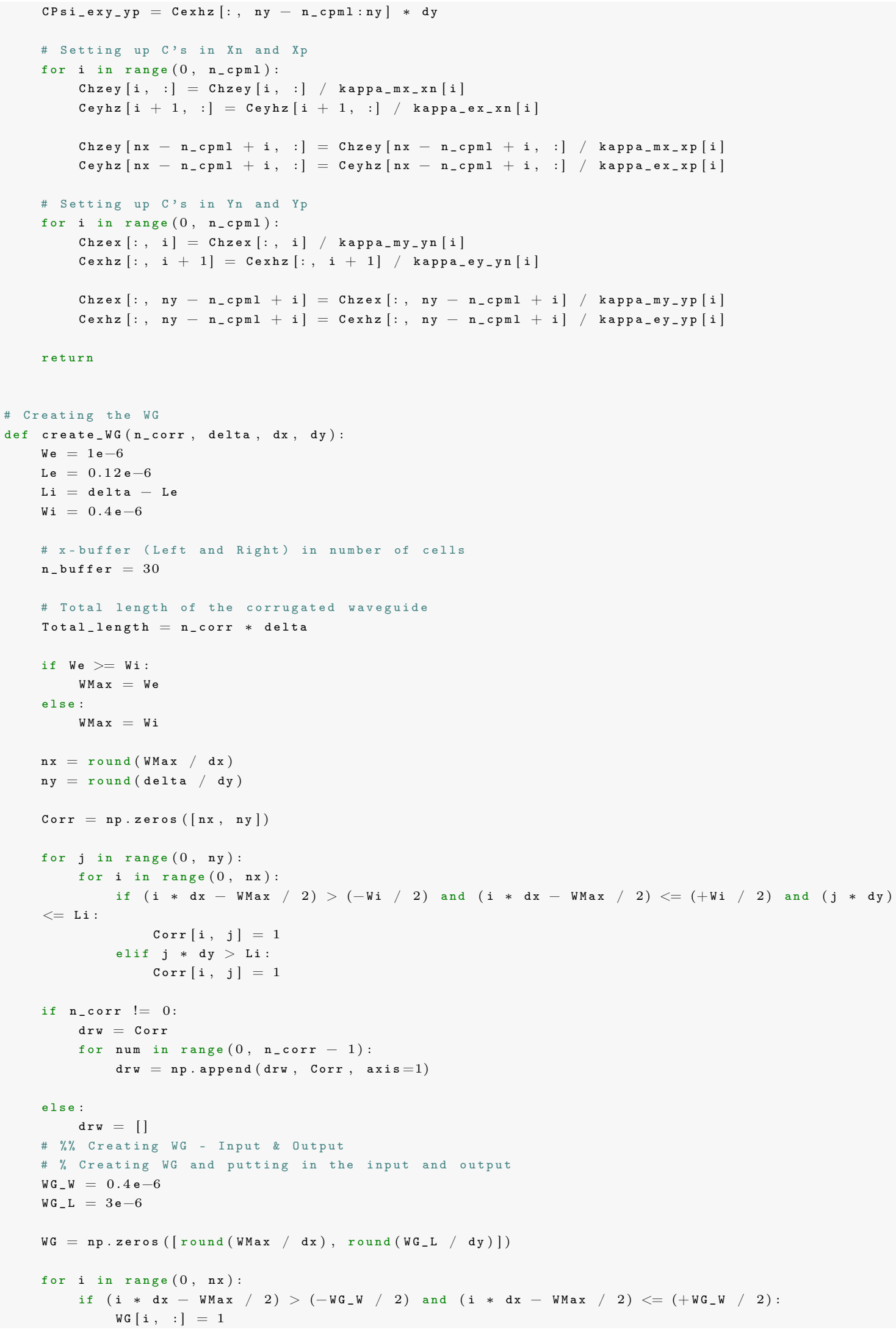




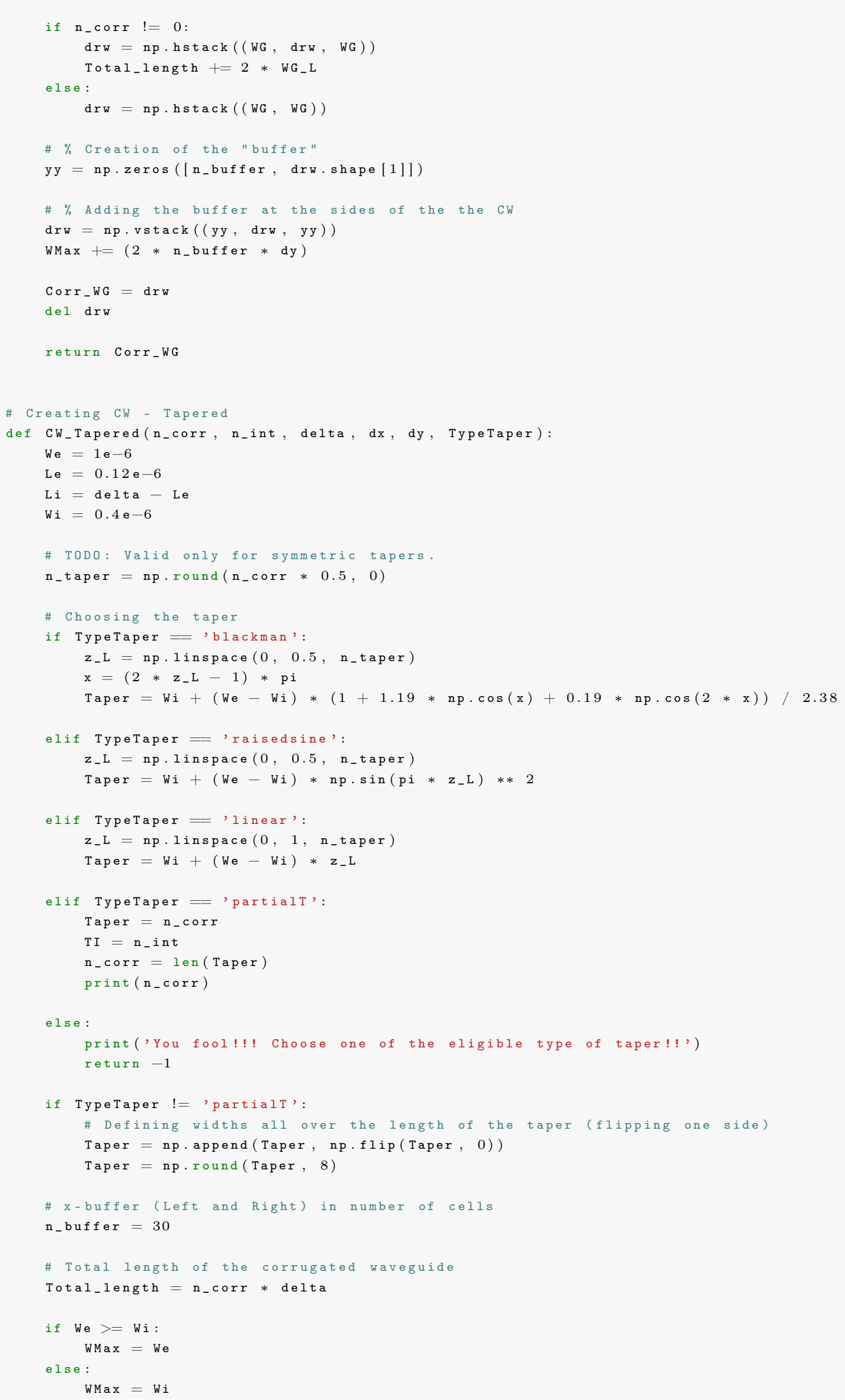




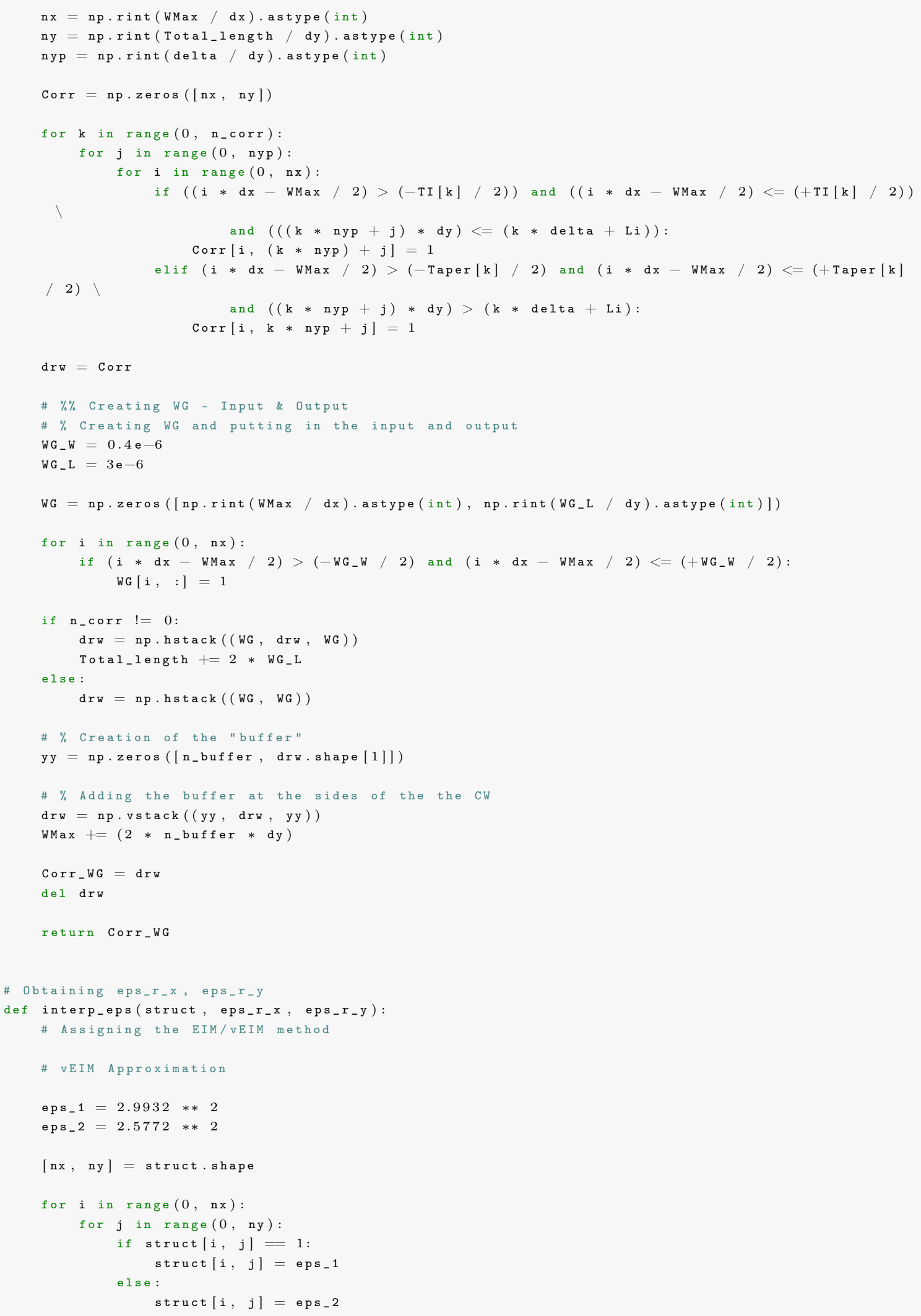




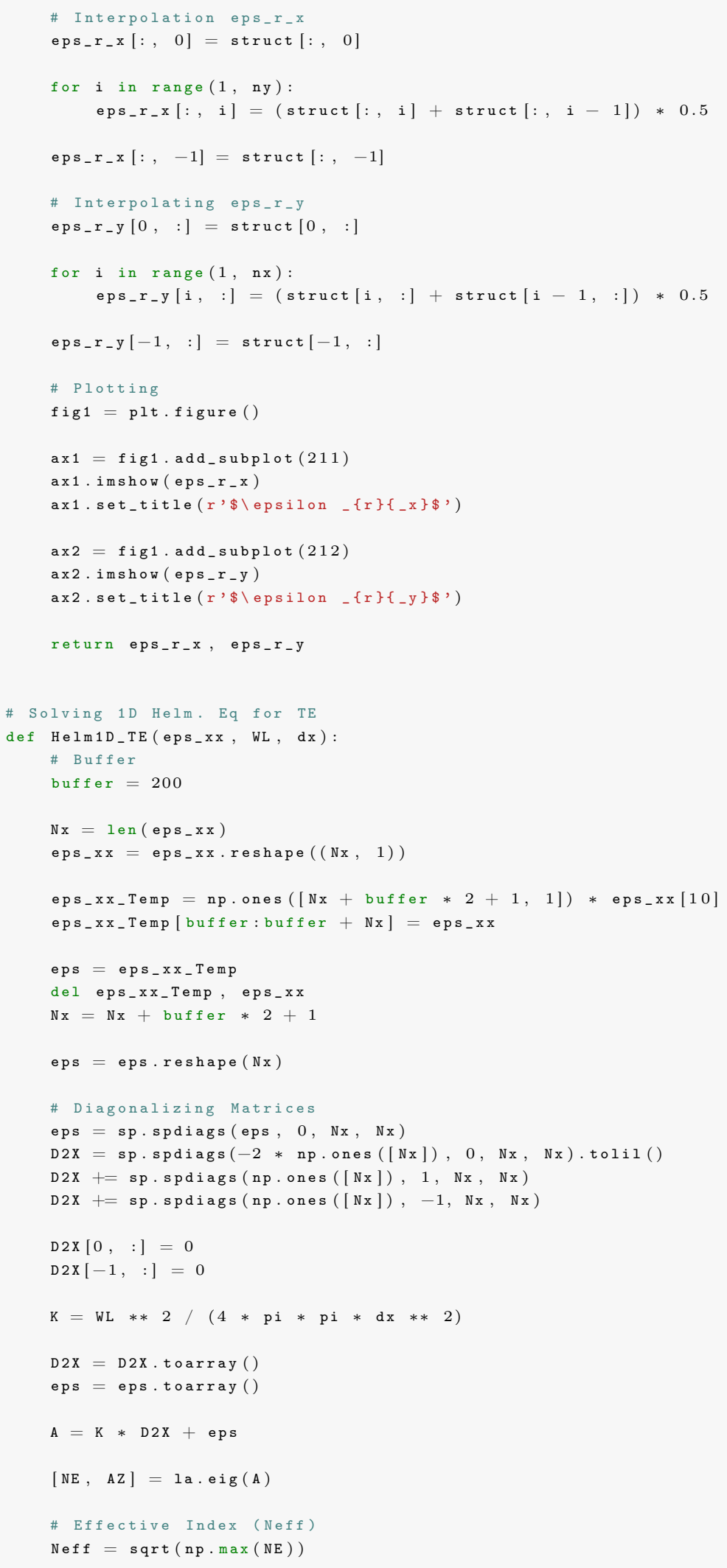




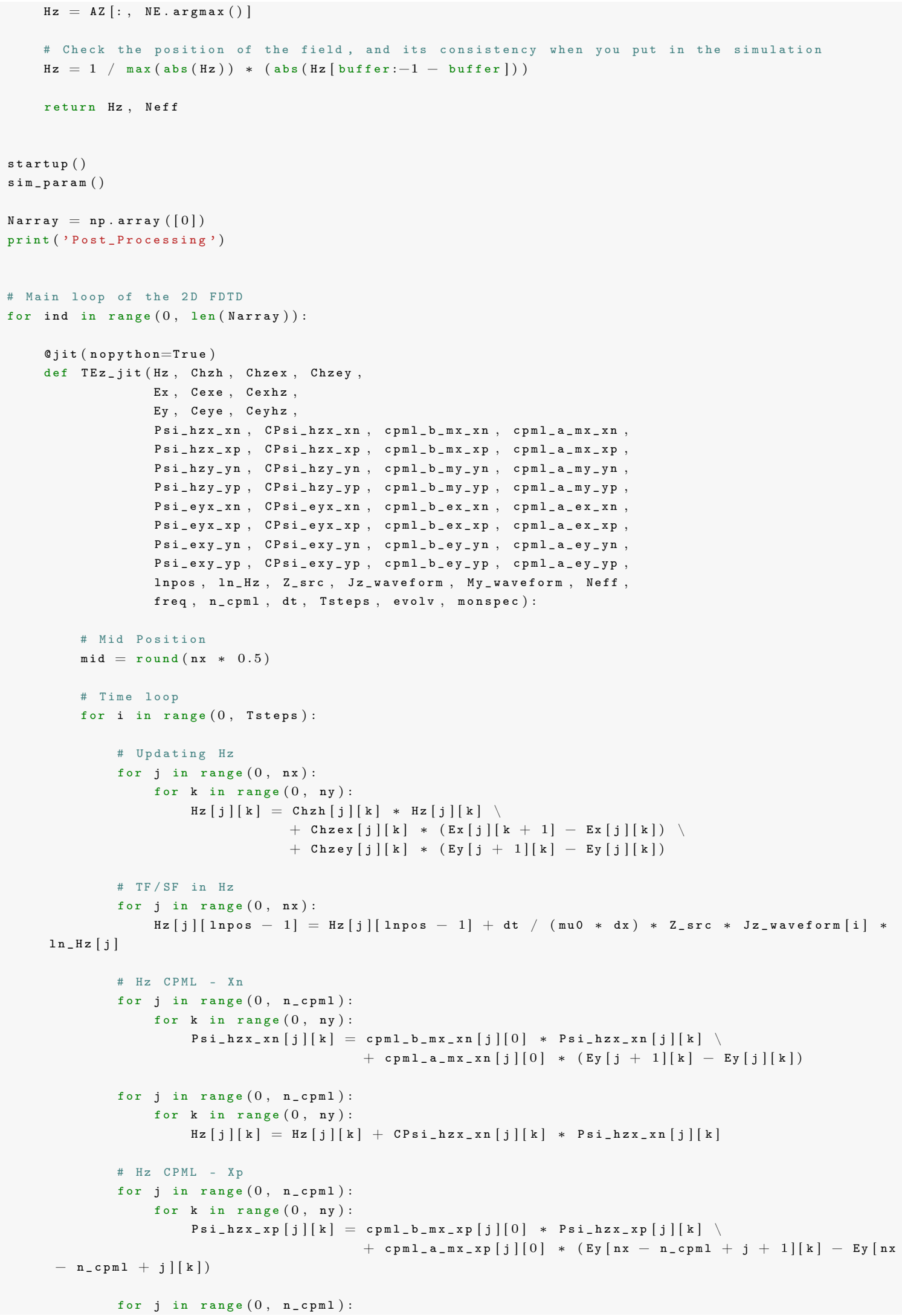




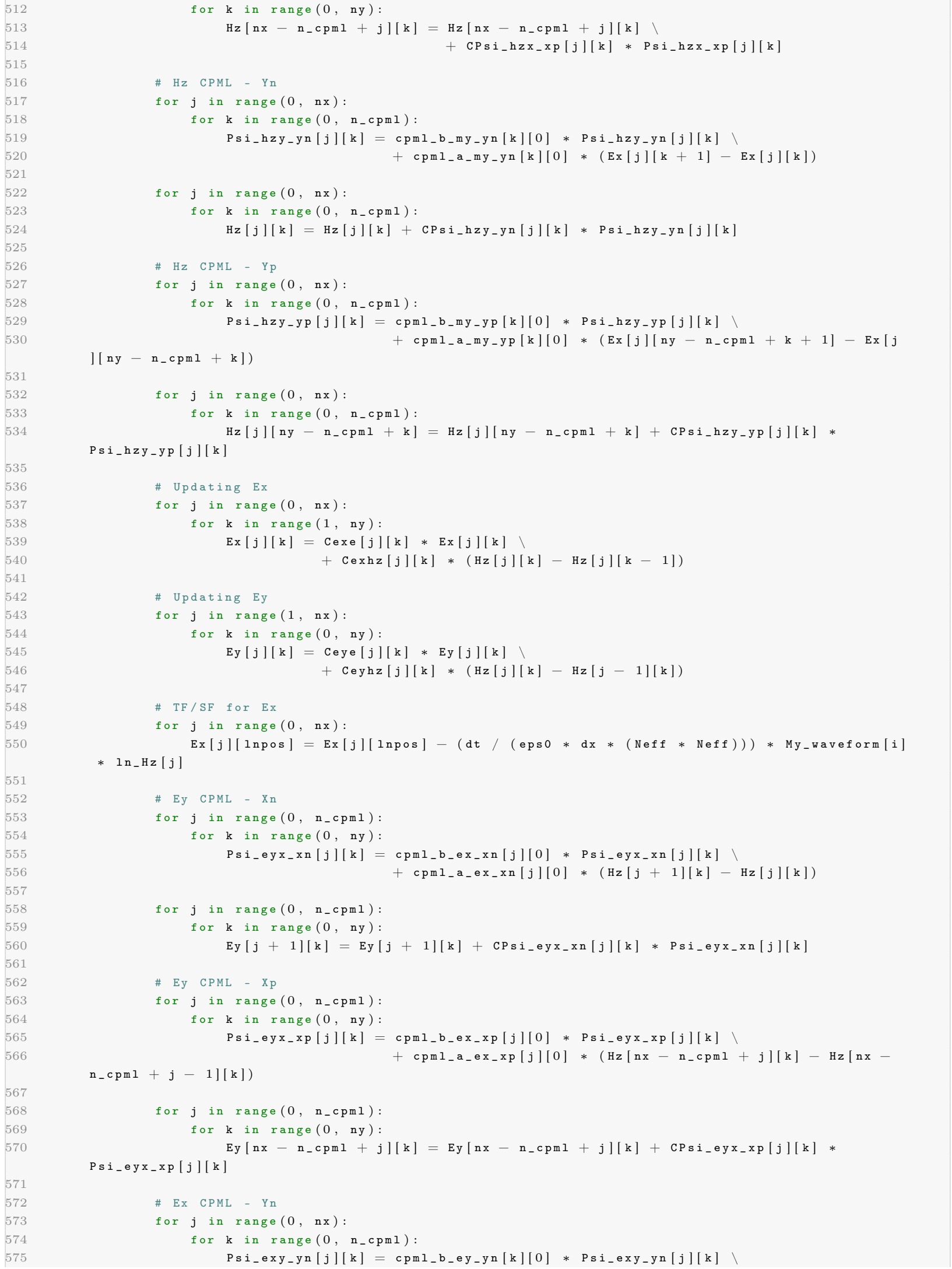




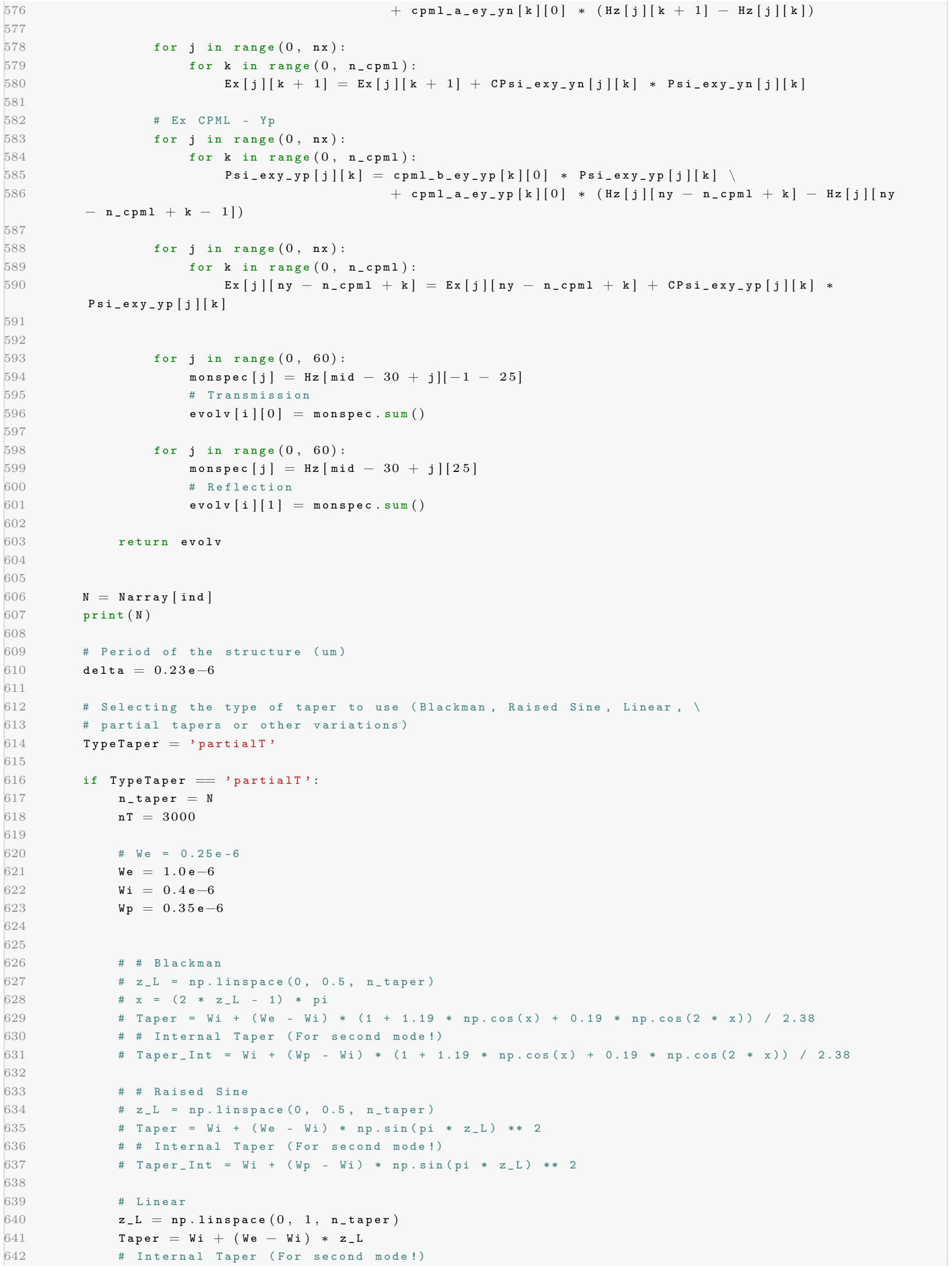




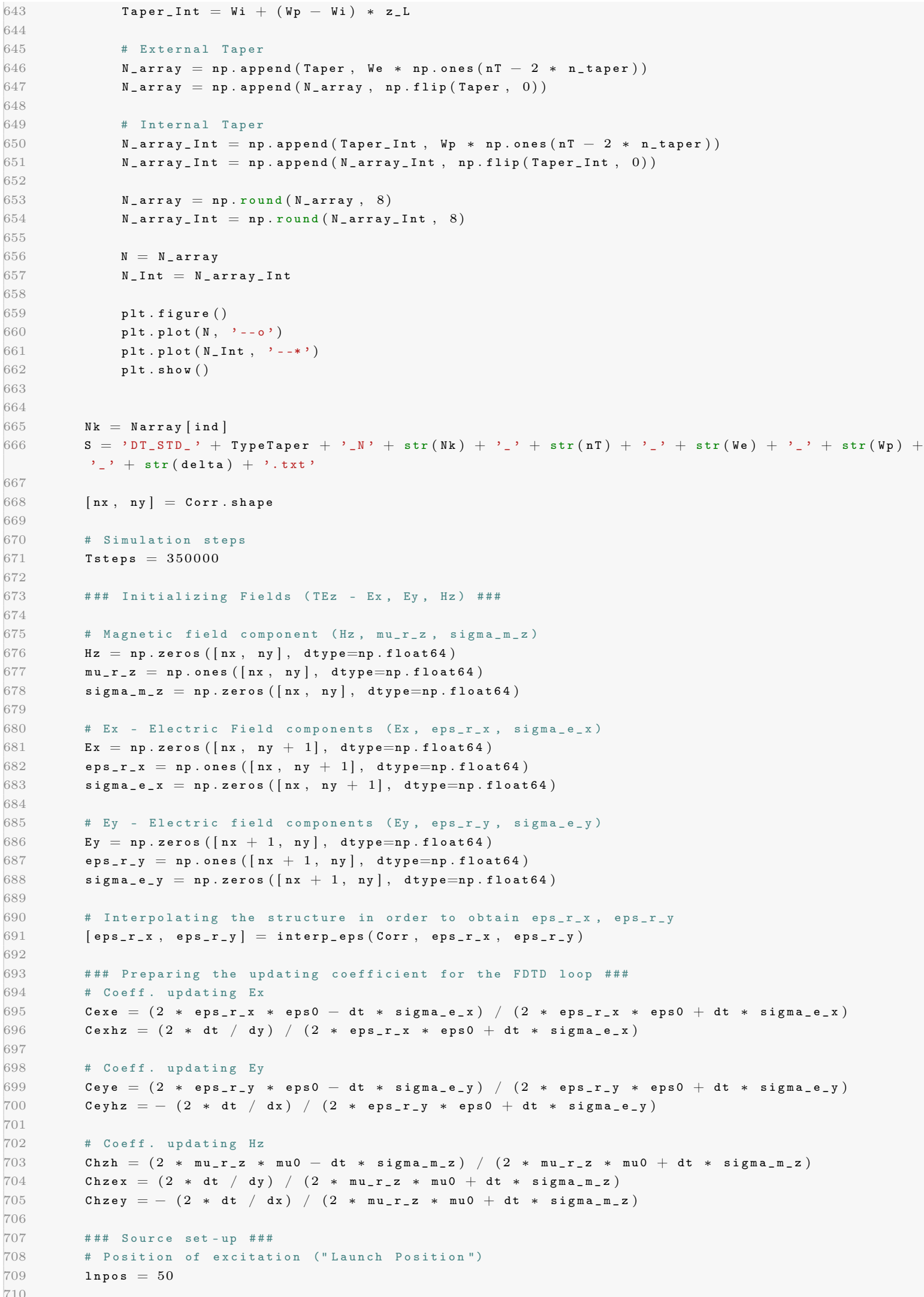




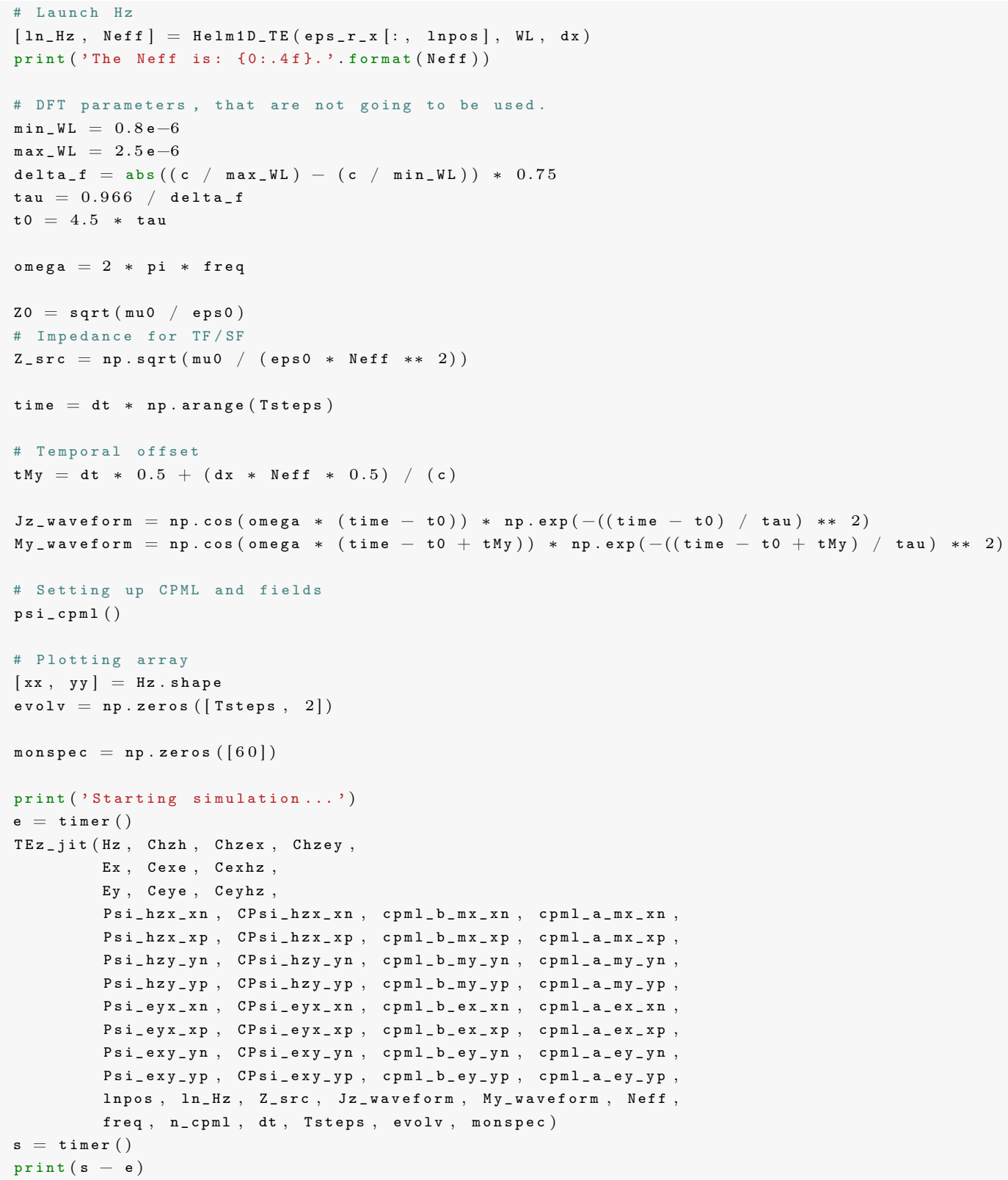

\section{Bibliography}

[1] Allen Taflove and Susan C Hagness. Computational electrodynamics: the finite-difference time-domain method. Artech house, 2005.

[2] Karl S Kunz and Raymond J Luebbers. The finite difference time domain method for electromagnetics. CRC press, 1993. 


\section{Appendix D}

\section{Mode Solver 2D}

This code, written in Python, is a 2D mode solver. The used algorithm is reported in [1, 2].

\section{D.1 Code Mode Solver}

Listing D.1: Code of the 2D Mode Solver

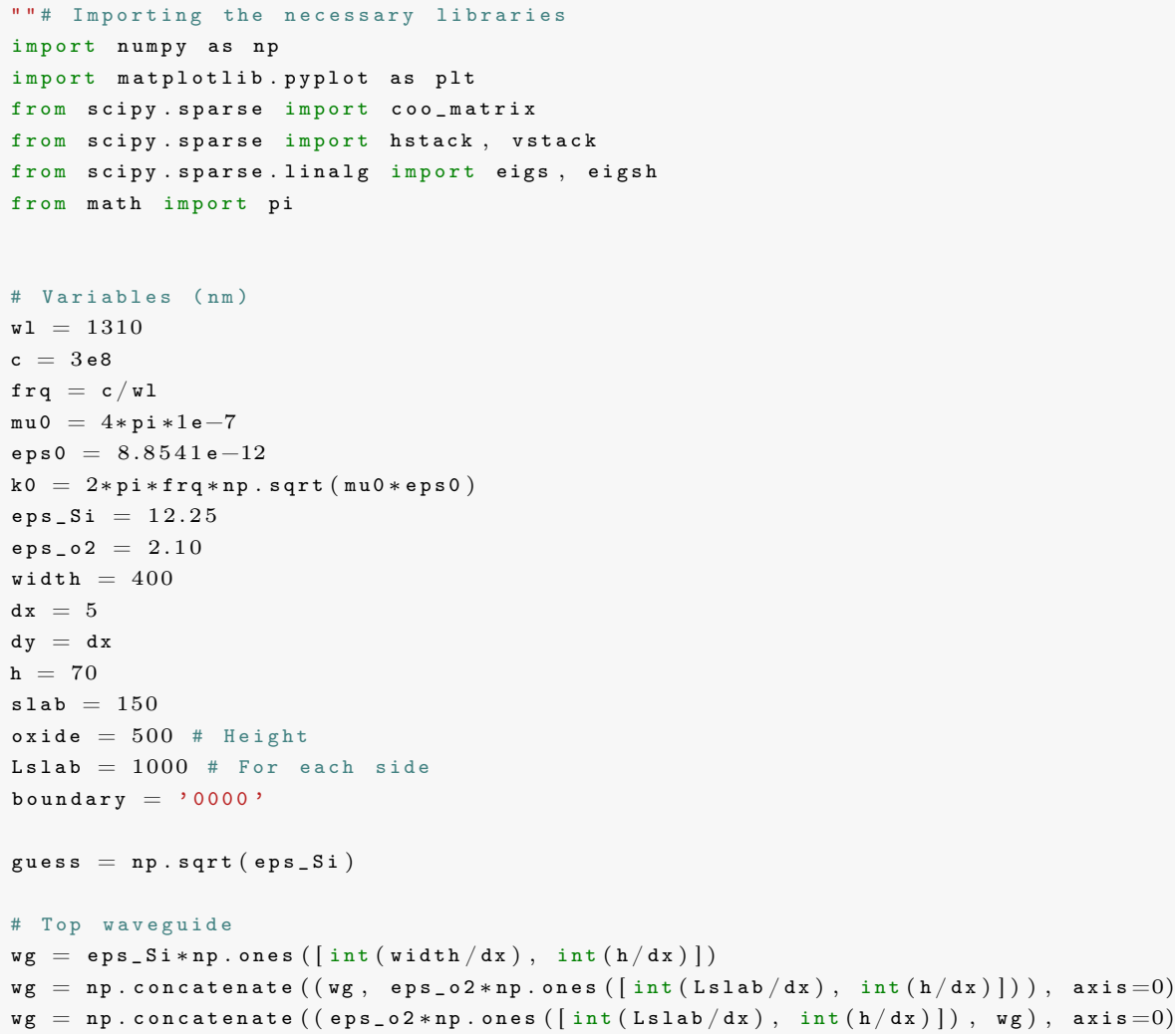




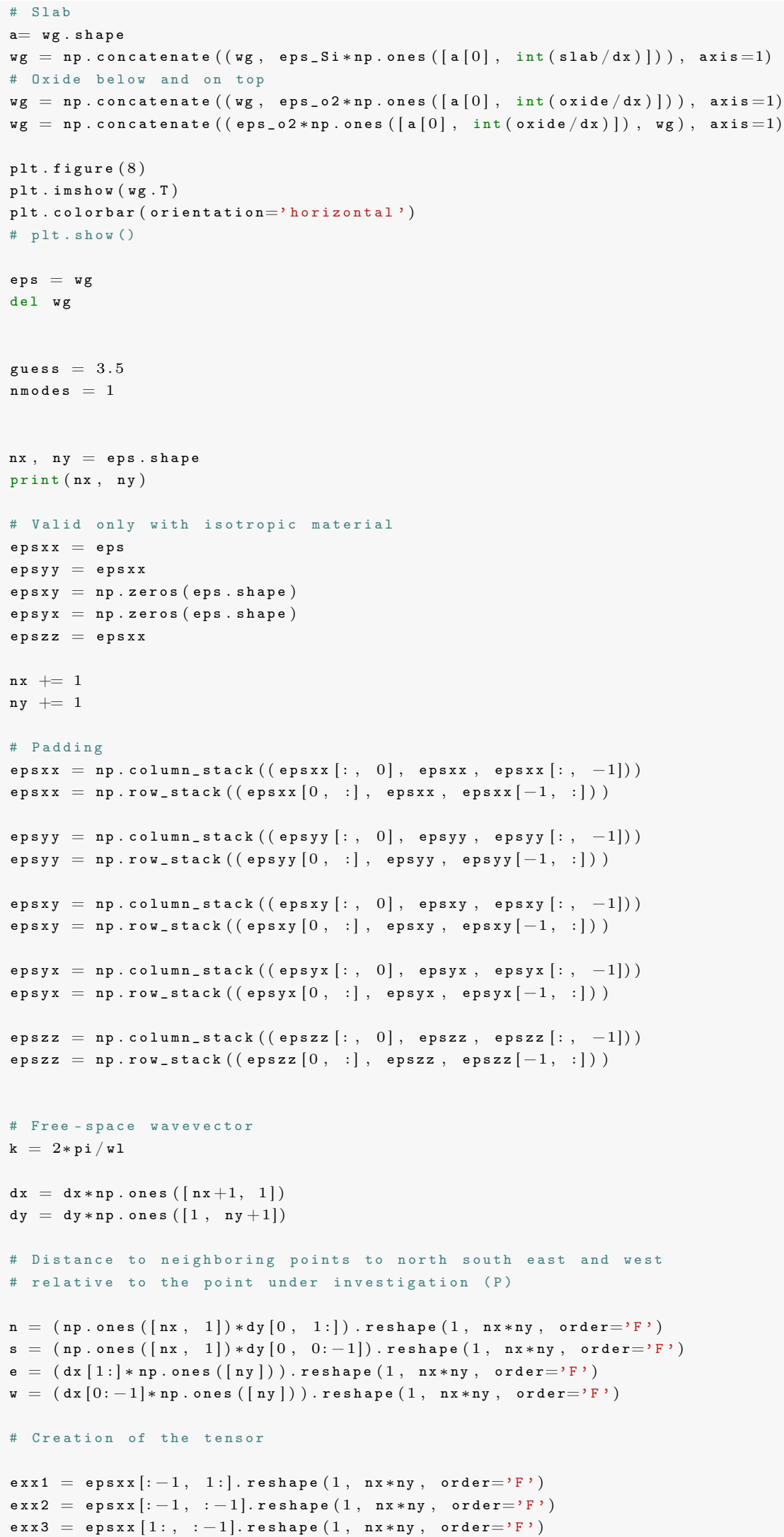




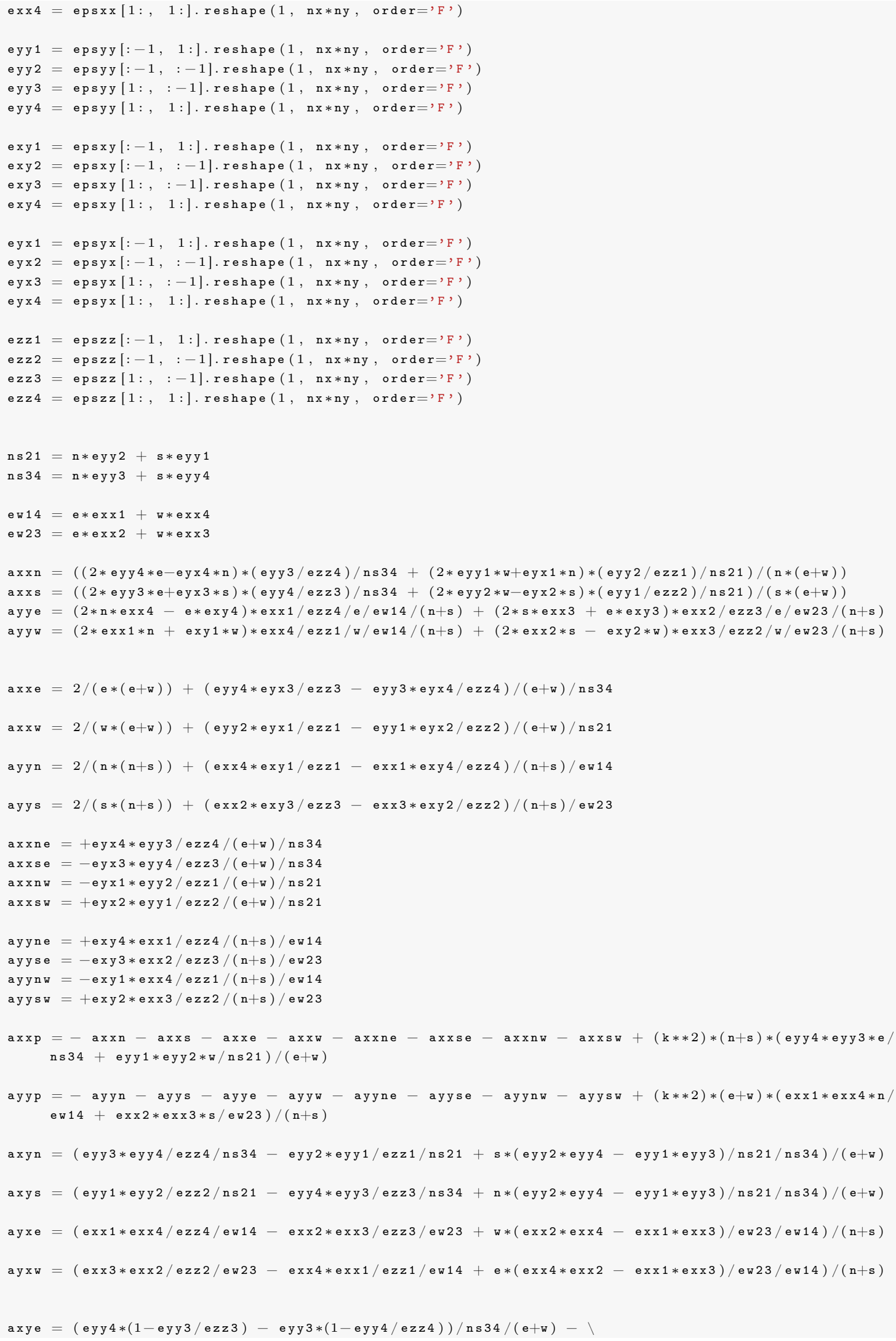




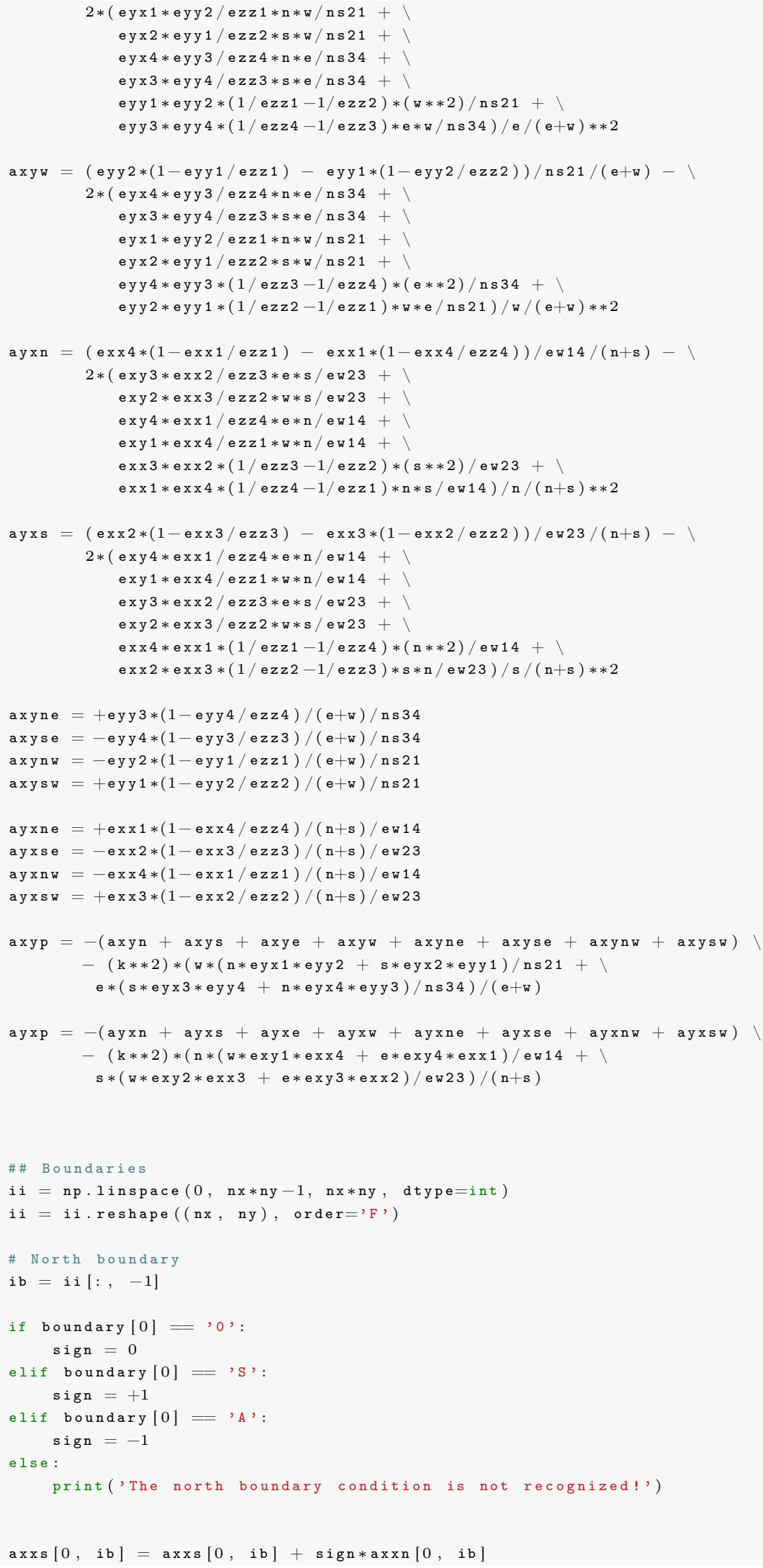




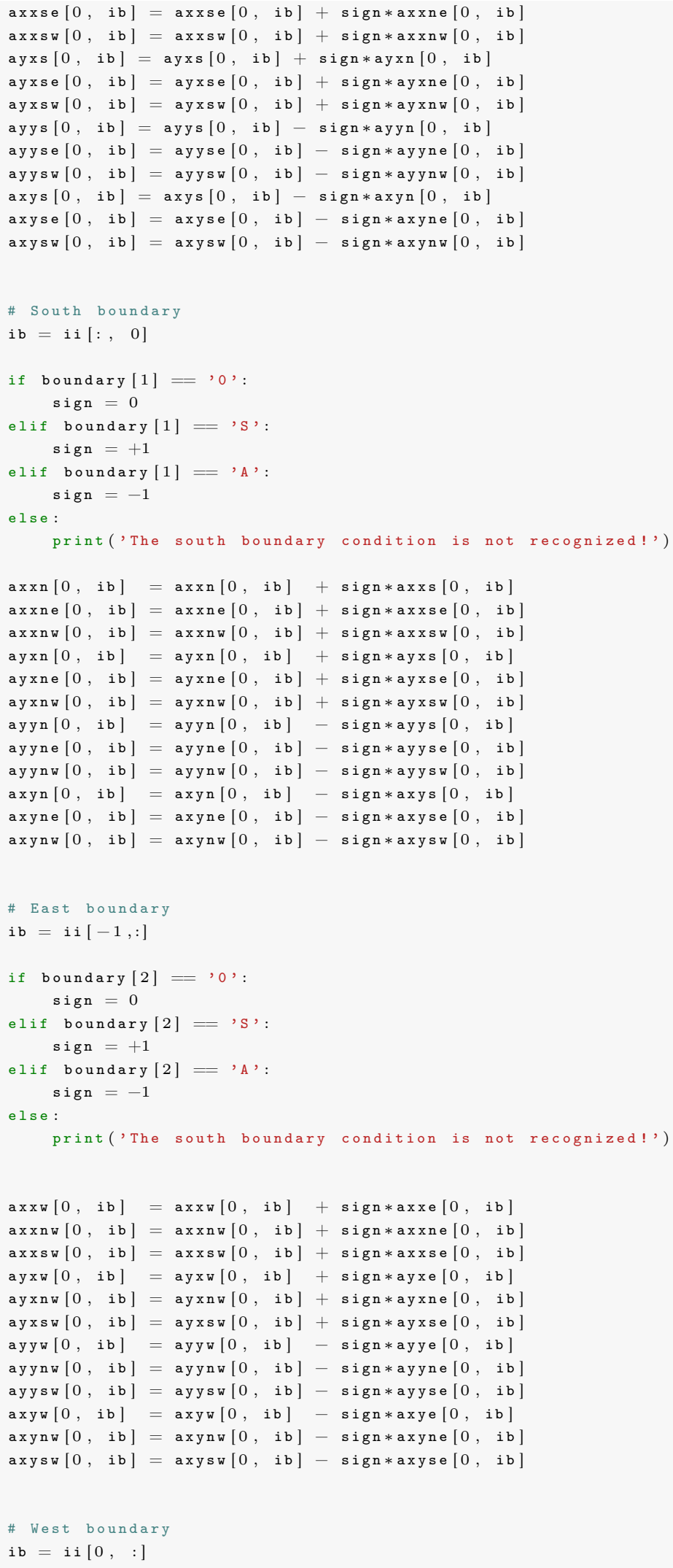




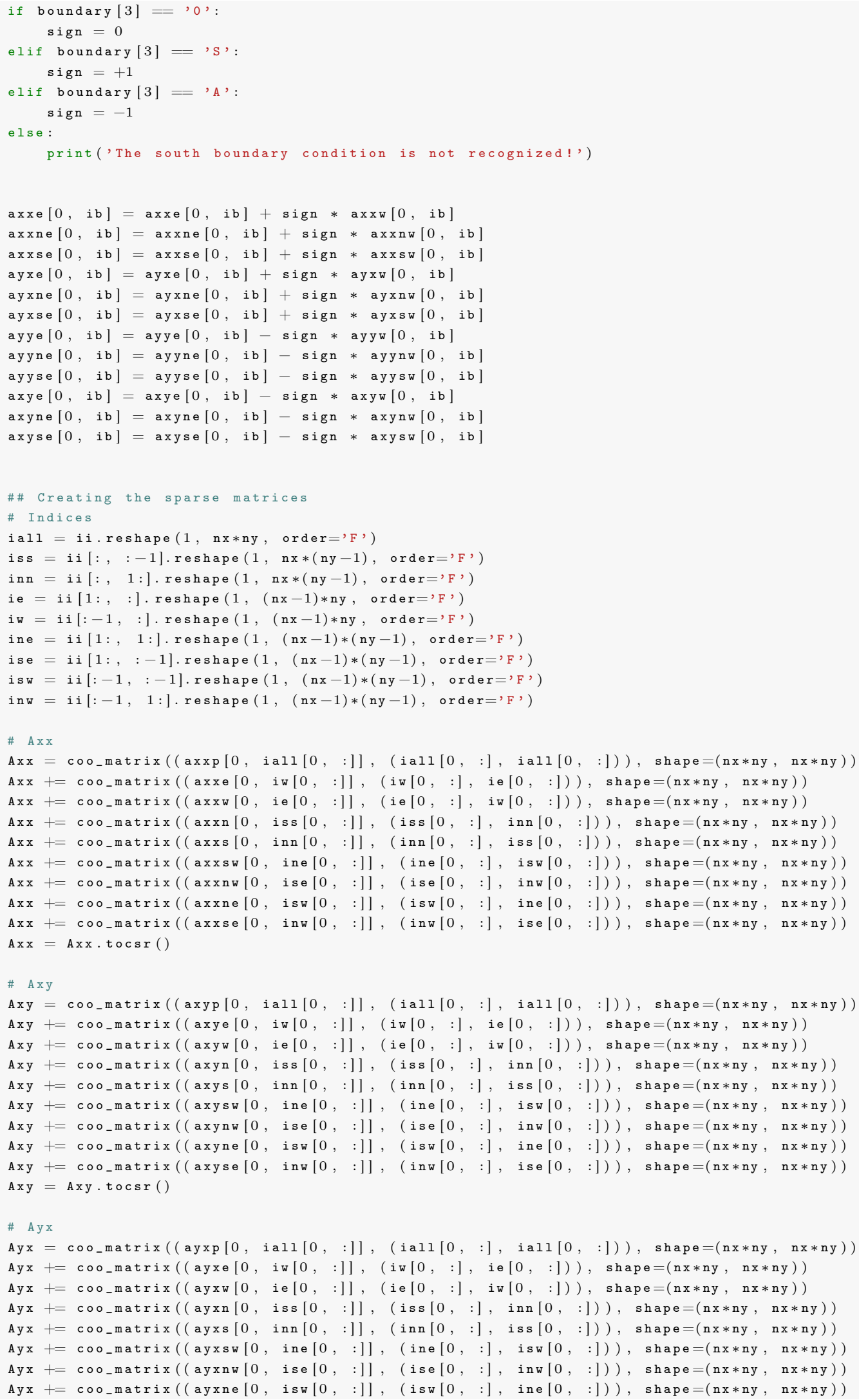




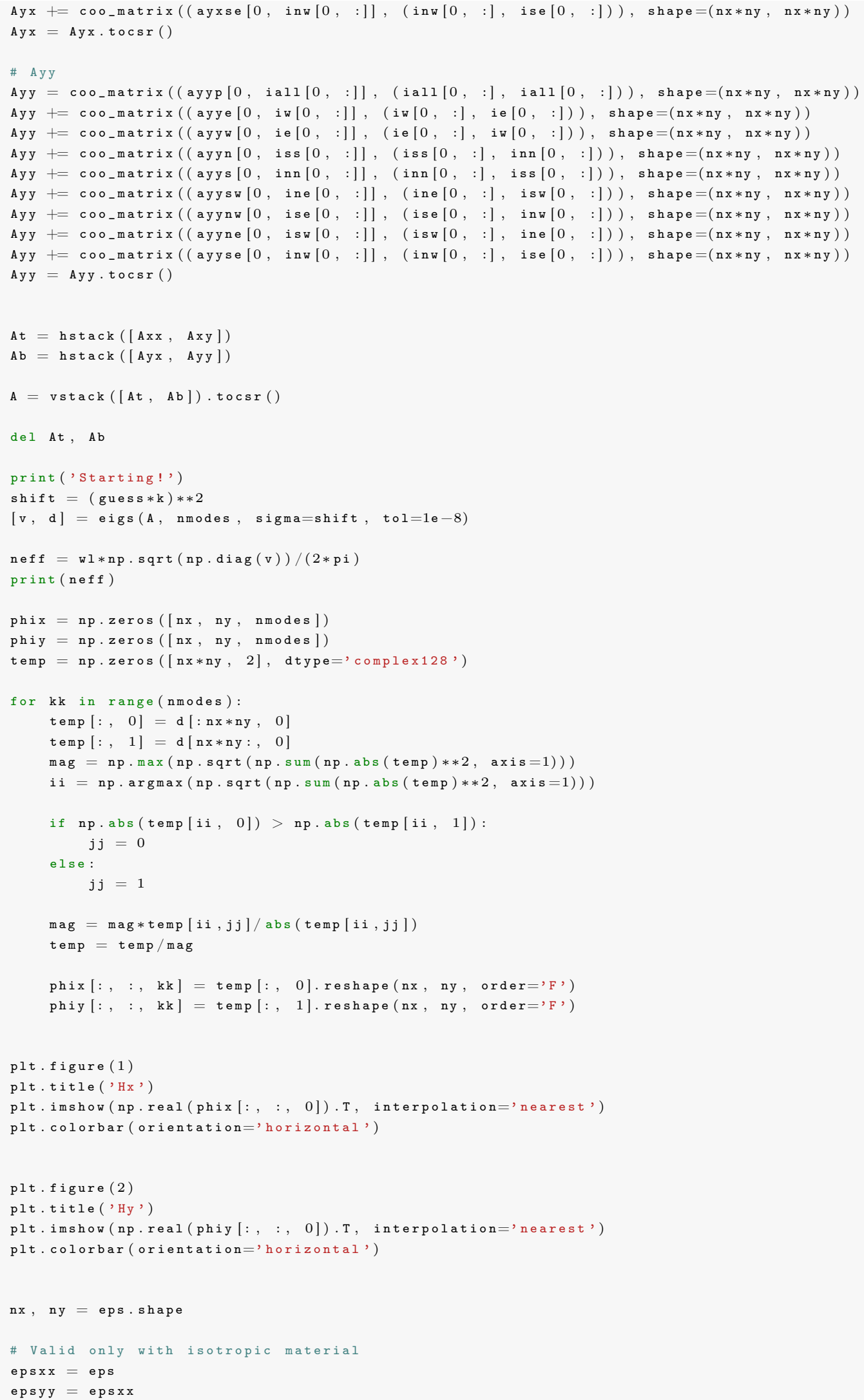




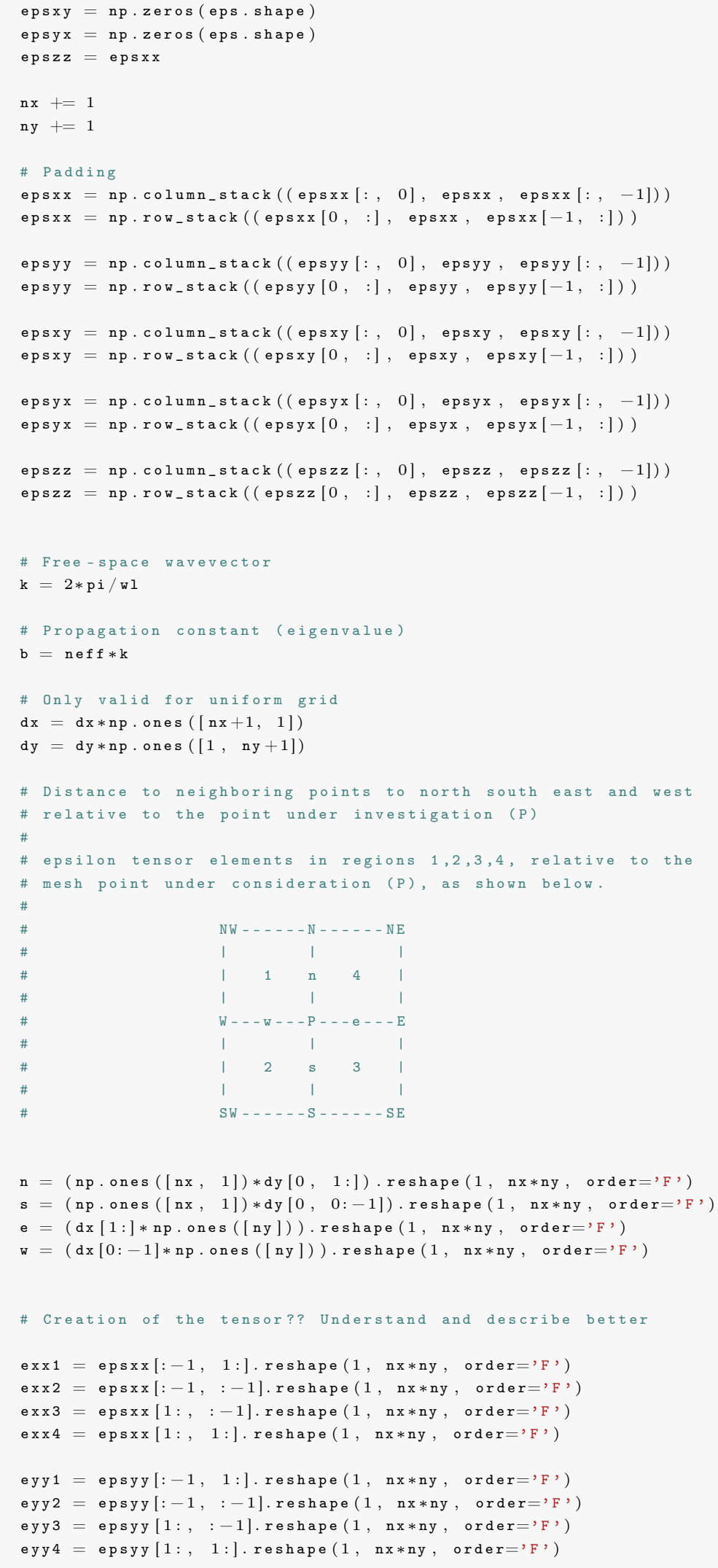




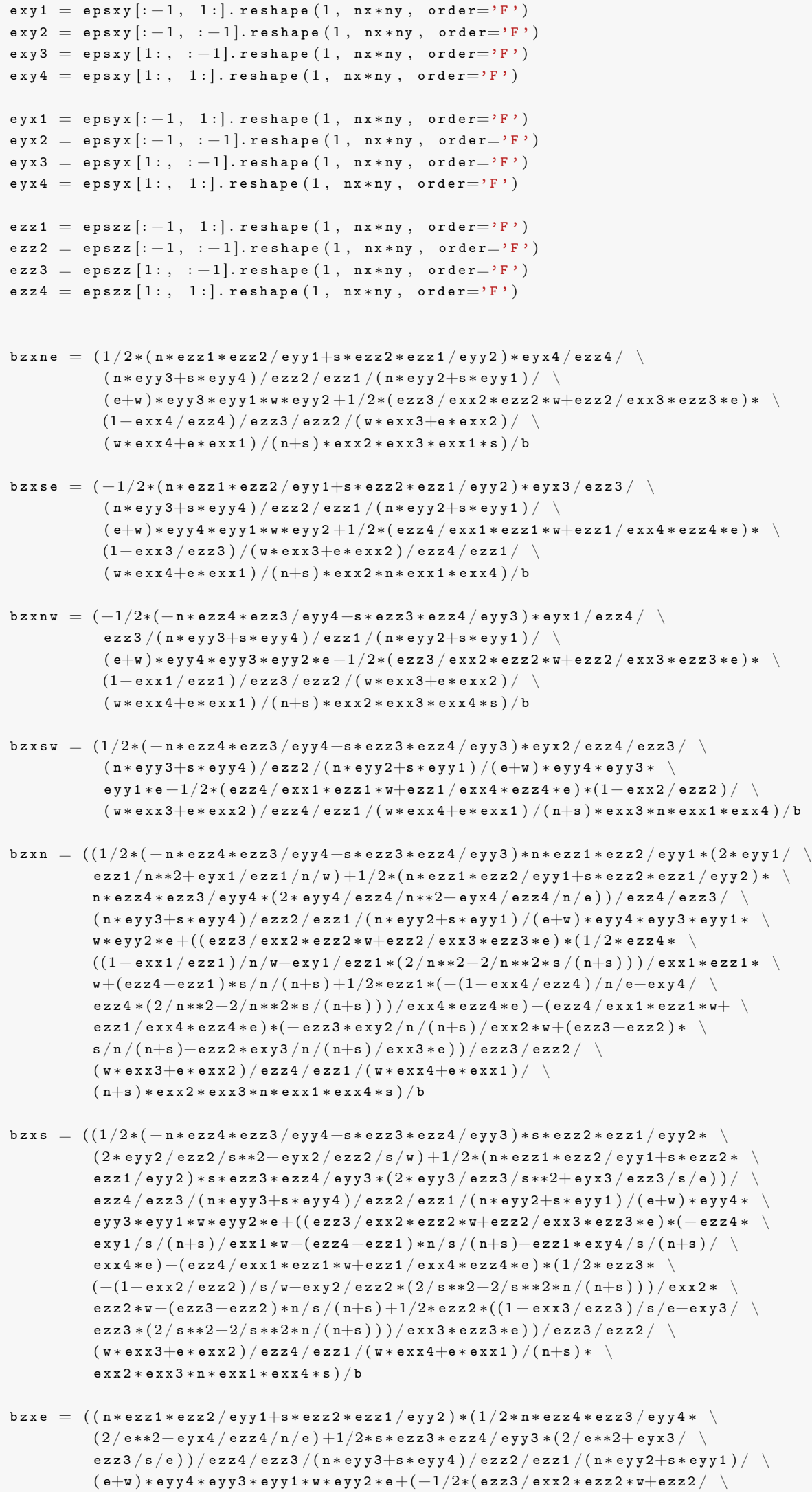




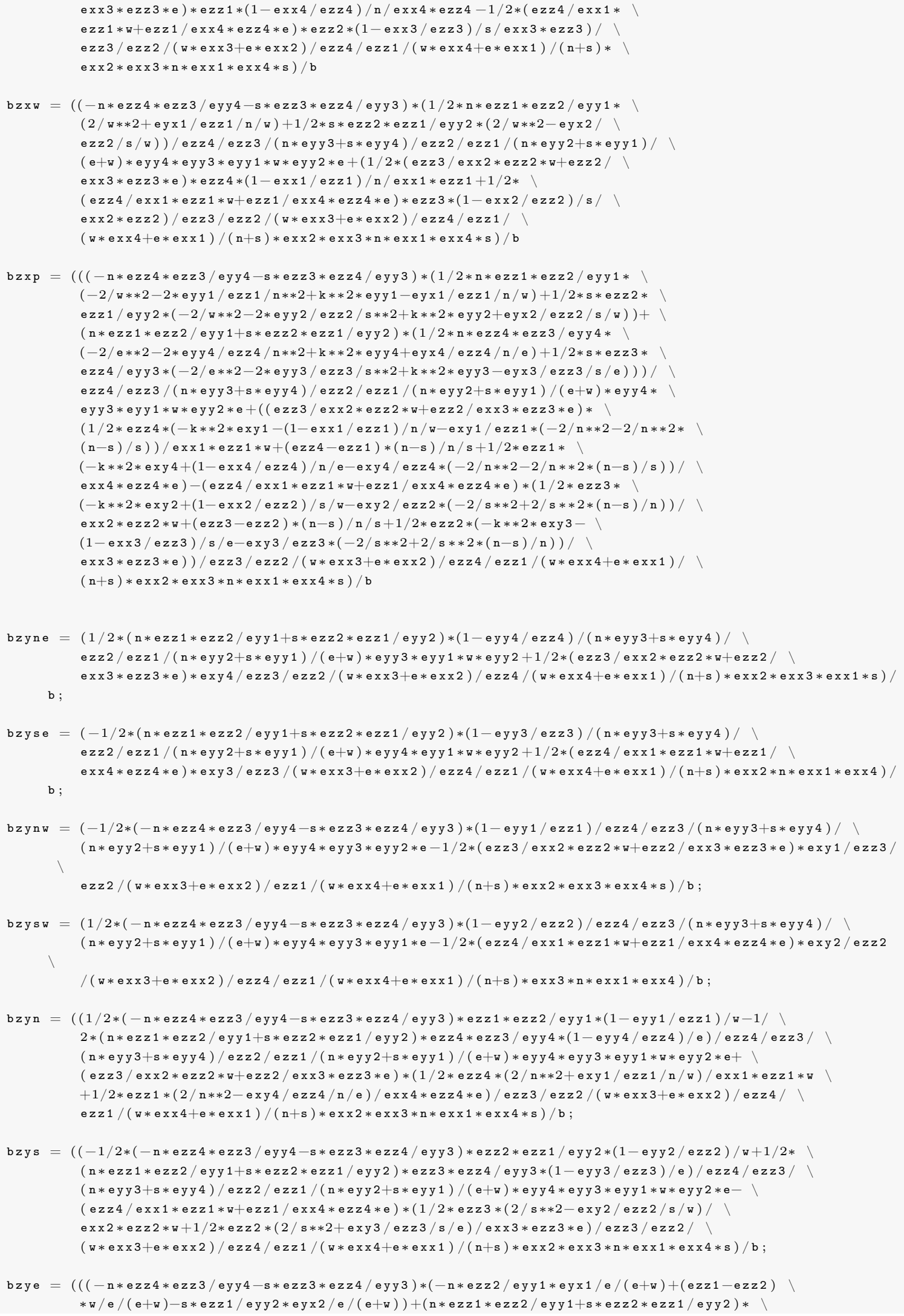


$(1 / 2 * \mathrm{n} *$ ezz $4 *$ ezz $3 /$ eyy $4 *(-(1-$ eyy $4 /$ ezz 4$) / \mathrm{n} / \mathrm{e}-$ eyx $4 /$ ezz $4 *(2 /$ e $* * 2-2 /$ e $* * 2 * w /(e+w)))+$

$1 / 2 * \mathrm{~s} *$ ezz $3 *$ ezz $4 /$ eyy $3 *((1-$ eyy $3 /$ ezz 3$) / \mathrm{s} / \mathrm{e}-$ eyx $3 /$ ezz $3 *(2 / \mathrm{e} * * 2-2 / \mathrm{e} * * 2 * \mathrm{w} /(\mathrm{e}+\mathrm{w})))$

$+($ ezz4 -ezz3 $) * w / e /(e+w))) /$ ezz $4 /$ ezz $3 /($ n*eyy $3+s *$ eyy 4$) /$ ezz $2 /$ ezz $1 /($ n $*$ ey y $2+s *$ eyy 1$)$

$/($ e + w $) *$ eу y $4 *$ eу y $3 *$ eу y $1 *$ w $*$ eуy $2 *$ e $+(1 / 2 *($ ezz $3 /$ exx $2 *$ ezz $2 *$ w + ezz $2 /$ exx $3 *$ ezz $3 *$ e $) *$ ezz $1 *)$

$(2 *$ exx $4 /$ ezz $4 /$ e $* * 2-$ exy $4 /$ ezz $4 / \mathrm{n} /$ e $) /$ exx $4 *$ ezz $4 * e-1 / 2 *($ ezz $4 /$ exx $1 *$ ezz $1 * w+e z z 1 / e x x 4 * e z z 4 * e)$

*ezz $2 *(2 *$ exx3/ezz3/e**2+exy3/ezz3/s/e)/exx3*ezz3*e)/ezz3/ezz2/(w*exx3+e*exx2)/

$\mathrm{ezz} 4 / \mathrm{ezz} 1 /(\mathrm{w} * \operatorname{exx} 4+\mathrm{e} * \operatorname{exx} 1) /(\mathrm{n}+\mathrm{s}) * \operatorname{exx} 2 * \operatorname{exx} 3 * \mathrm{n} * \operatorname{exx} 1 * \operatorname{exx} 4 * \mathrm{~s}) / \mathrm{b}$

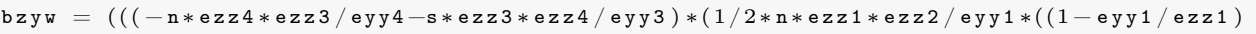

$/ \mathrm{n} / \mathrm{w}-\mathrm{eyx} 1 / \mathrm{ezz} 1 *(2 / \mathrm{w} * * 2-2 / \mathrm{w} * * 2 * \mathrm{e} /(\mathrm{e}+\mathrm{w})))-(\mathrm{ezz} 1-\mathrm{ezz} 2) * \mathrm{e} / \mathrm{w} /(\mathrm{e}+\mathrm{w})+1 / 2 * \mathrm{~s} * \mathrm{ezz} 2 * \mathrm{ezz} 1 /$ ey y $2 *(-(1-$ ey $2 /$ ezz 2$) / \mathrm{s} / \mathrm{w}-$ eyx $2 /$ ezz $2 *(2 / \mathrm{w} * * 2-2 / \mathrm{w} * * 2 *$ e $/($ e + w $))))+(\mathrm{n} *$ ezz $1 *$ ezz $2 /$ ey y $1+\mathrm{s} *$ ezz $2 *$ ezz $1 /$ eyy 2$) *(-n * e z z 3 /$ ey $4 *$ eyx $4 / w /(e+w)-s * e z z 4 /$ ey $3 *$ eyx $3 / w /(e+w)-(e z z 4-e z z 3) * e / w /$ $($ e+w $))) / e z z 4 /$ ezz $3 /(\mathrm{n} *$ ey $3+\mathrm{s} *$ eyy 4$) /$ ezz $2 /$ ezz $1 /(\mathrm{n} *$ eyy $2+\mathrm{s} *$ eyy 1$) /($ e+w $) *$ eyy $4 *$ eyy $3 *$ eyy $1 *$

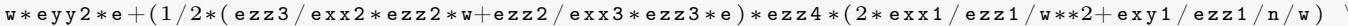
/exx $1 *$ ezz $1 * \mathrm{w}-1 / 2 *(\mathrm{ezz} 4 / \mathrm{exx} 1 * \mathrm{ezz} 1 * \mathrm{w}+\mathrm{ezz} 1 / \mathrm{exx} 4 * \mathrm{ezz} 4 * \mathrm{e}) * \mathrm{ezz} 3 *(2 * \mathrm{exx} 2 / \mathrm{ezz} 2 / \mathrm{w} * * 2-\mathrm{exy} 2 /$ ezz2/s/w)/exx2*ezz2*w)/ezz3/ezz2/(w*exx3+e*exx2)/ezz4/ezz1/(w*exx4+e*exx1)/ $(\mathrm{n}+\mathrm{s}) * \operatorname{ex} \times 2 * \operatorname{ex} \times 3 * \mathrm{n} * \operatorname{ex} \times 1 * \operatorname{ex} \times 4 * \mathrm{~s}) / \mathrm{b}$

bzyp $=(((-\mathrm{n} *$ ezz $4 *$ ezz $3 /$ eyy $4-\mathrm{s} *$ ezz $3 *$ ezz $4 /$ ey y 3$) *(1 / 2 * \mathrm{n} *$ ezz $1 *$ ezz $2 /$ ey $1 *(-\mathrm{k} * * 2 *$ eyx $1-)$ $(1-$ ey $1 /$ ezz 1$) / \mathrm{n} / \mathrm{w}-\mathrm{eyx} 1 / \mathrm{ezz} 1 *(-2 / \mathrm{w} * * 2+2 / \mathrm{w} * * 2 *(\mathrm{e}-\mathrm{w}) / \mathrm{e}))+(\mathrm{ezz} 1-\mathrm{ezz} 2) *(\mathrm{e}-\mathrm{w}) / \mathrm{e} / \mathrm{w}+$ $1 / 2 * \mathrm{~s} *$ ezz $2 *$ ezz $1 /$ ey $2 *(-\mathrm{k} * * 2 *$ eyx $2+(1-$ ey $2 /$ ezz 2$) / \mathrm{s} / \mathrm{w}-$ eyx $2 /$ ezz $2 *(-2 / \mathrm{w} * * 2+2 / \mathrm{w} * * 2 *$ $(\mathrm{e}-\mathrm{w}) / \mathrm{e})))+(\mathrm{n} *$ ezz $1 *$ ezz $2 /$ ey y $1+\mathrm{s} *$ ezz $2 *$ ezz $1 /$ ey y 2$) *(1 / 2 * \mathrm{n} *$ ezz $4 *$ ezz $3 /$ ey y $4 *$ $(-\mathrm{k} * * 2 *$ eyx $4+(1-$ ey $4 /$ ezz 4$) / \mathrm{n} / \mathrm{e}-\mathrm{eyx} 4 / \mathrm{ezz} 4 *(-2 / \mathrm{e} * * 2-2 / \mathrm{e} * * 2 *(\mathrm{e}-\mathrm{w}) / \mathrm{w}))+1 / 2 * \mathrm{~s} * \mathrm{ezz} 3 * \backslash$ ezz $4 /$ eуy $3 *(-\mathrm{k} * * 2 *$ eyx $3-(1-$ ey $3 /$ ezz 3$) / \mathrm{s} / \mathrm{e}-$ eyx $3 /$ ezz $3 *(-2 /$ e $* * 2-2 /$ e $* * 2 *($ e $-w) / w))+\backslash$ $($ ezz4-ezz3 $) *(e-w) / e / w)) / e z z 4 / e z z 3 /($ n*eyy3+s*eyy 4$) / e z z 2 / e z z 1 /($ n*eyy $2+s * e y y 1) /$ $($ e+w $) *$ ey $4 *$ ey $3 *$ ey $1 *$ w $*$ ey $2 *$ e $+(($ ezz $3 /$ exx $2 *$ ezz $2 * w+e z z 2 / e x x 3 *$ ezz $3 * e) *(1 / 2 *$ ezz $4 *)$ $(-2 / \mathrm{n} * * 2-2 * \operatorname{exx} 1 / \mathrm{ezz} 1 / \mathrm{w} * * 2+\mathrm{k} * * 2 * \operatorname{exx} 1-\operatorname{exy} 1 / \mathrm{ezz} 1 / \mathrm{n} / \mathrm{w}) / \mathrm{exx} 1 * \operatorname{ezz} 1 * \mathrm{w}+1 / 2 * \operatorname{ezz} 1 *$ $(-2 / \mathrm{n} * * 2-2 * \operatorname{exx} 4 /$ ezz $4 /$ e $* * 2+\mathrm{k} * * 2 *$ exx $4+\operatorname{exy} 4 /$ ezz $4 / \mathrm{n} / \mathrm{e}) / \mathrm{exx} 4 * \mathrm{ezz} 4 * \mathrm{e})-\backslash$ $($ ezz4/exx $1 *$ ezz $1 *$ w + ezz1 /exx $4 *$ ezz $4 *$ e $) *(1 / 2 *$ ezz $3 *(-2 / \mathrm{s} * * 2-2 *$ exx $2 /$ ezz $2 / \mathrm{w} * * 2+$ $\mathrm{k} * * 2 *$ exx $2+$ exy $2 /$ ezz $2 / \mathrm{s} / \mathrm{w}) / \mathrm{exx} 2 *$ e zz $2 * \mathrm{w}+1 / 2 *$ ezz $2 *(-2 / \mathrm{s} * * 2-2 * \operatorname{exx} 3 / \mathrm{ezz} 3 / \mathrm{e} * * 2+\mathrm{k} * * 2 * \operatorname{exx} 3$ -exy3/ezz3/s/e)/exx3*ezz3*e) )/ezz3/ezz2/(w*exx3+e*exx2)/ezz4/ezz1/(w*exx4+e*exx1)/ $(\mathrm{n}+\mathrm{s}) * \operatorname{ex} \times 2 * \operatorname{ex} \times 3 * \mathrm{n} * \operatorname{ex} \times 1 * \operatorname{exx} 4 * \mathrm{~s}) / \mathrm{b}$

\# \# Boundaries

i $\mathrm{i}=\mathrm{np} . \mathrm{linspace}(0, \mathrm{nx} * \mathrm{ny}-1, \mathrm{nx} * \mathrm{ny}, \mathrm{dtype}=\mathrm{int})$

ii $=i i \cdot r e s h a p e\left((n x, n y)\right.$, order $\left.={ }^{\prime} F '\right)$

\# North boundary

ib $=$ i $[:, \quad-1]$

if boundary [0] $=$, 0 ':

$$
\text { sign }=0
$$

elif boundary $[0]==$ ' $S$ ':

sign $=+1$

elif boundary $[0]==$, A, :

$$
\operatorname{sign}=-1
$$

else :

print ('The north boundary condition is not recognized!')

$\mathrm{bzxs}[0, \mathrm{ib}]=\mathrm{bzxs}[0, \mathrm{ib}]+\operatorname{sign} * \mathrm{bzxn}[0, i b]$ $\mathrm{bzxse}[0, \mathrm{ib}]=\mathrm{bzxse}[0, i b]+\operatorname{sign} * \mathrm{bzxne}[0, i b]$ $\mathrm{bzxsw}[0, \mathrm{ib}]=\mathrm{bzxsw}[0, i b]+\operatorname{sign} * \mathrm{bzxnw}[0, i b]$ bzys $[0$, ib $]=$ bzys $[0, i b]-\operatorname{sign*bzyn}[0, i b]$ bzyse $[0$, ib $]=$ bzyse $[0$, ib $]-\operatorname{sign*bzyne}\left[\begin{array}{ll}0, & i b\end{array}\right]$ bzysw $[0, i b]=\operatorname{bzysw}[0, i b]-\operatorname{sign*bzynw}[0, i b]$

\# South boundary

$i b=i i[:, 0]$

if boundary [1] $==$, 0 , :

sign $=0$

elif boundary [1] = ' $\mathrm{S}$ ' :

sign $=+1$

elif boundary [1] = 'A, : else :

$$
\text { sign }=-1
$$

print ('The south boundary condition is not recognized!') 


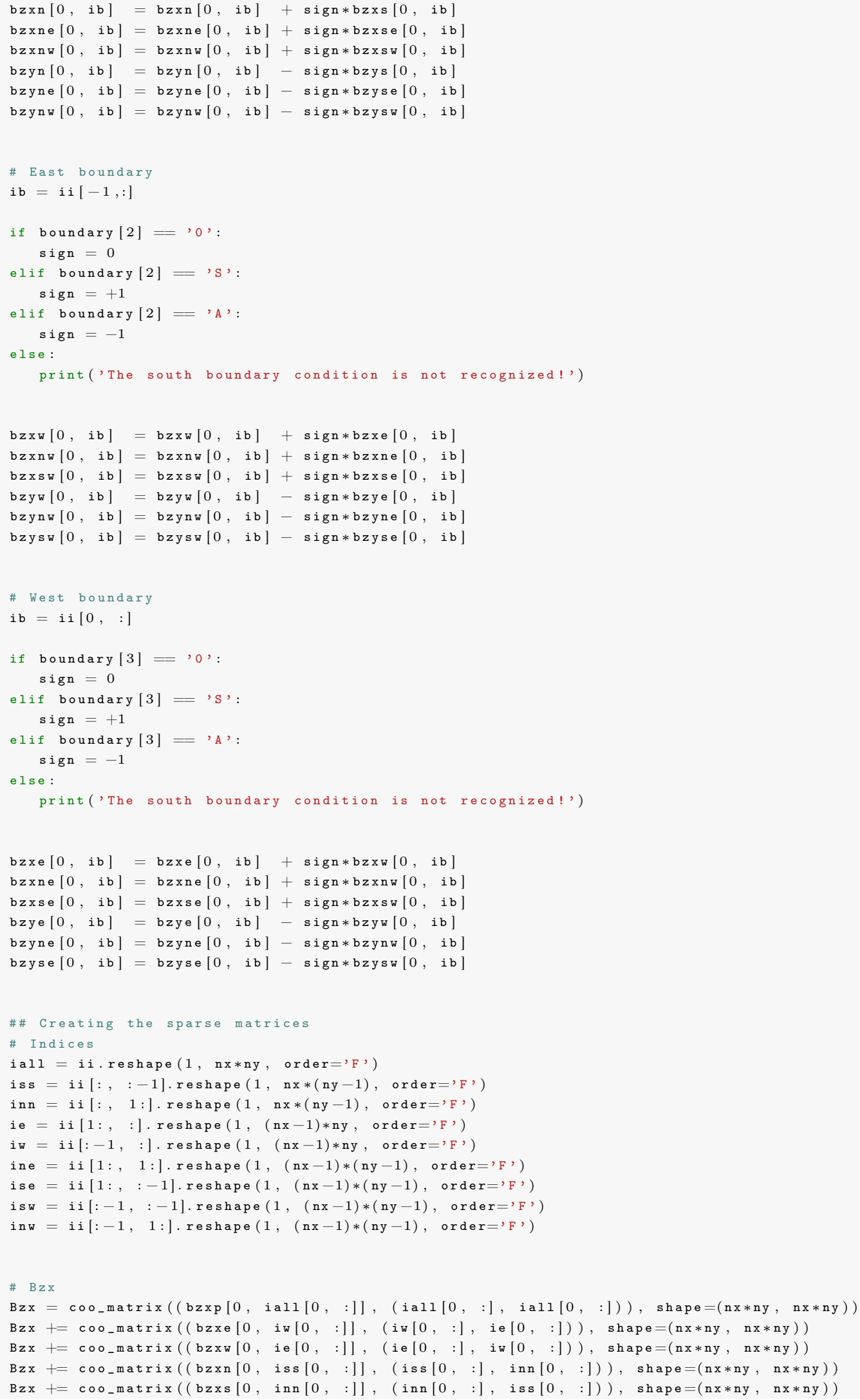




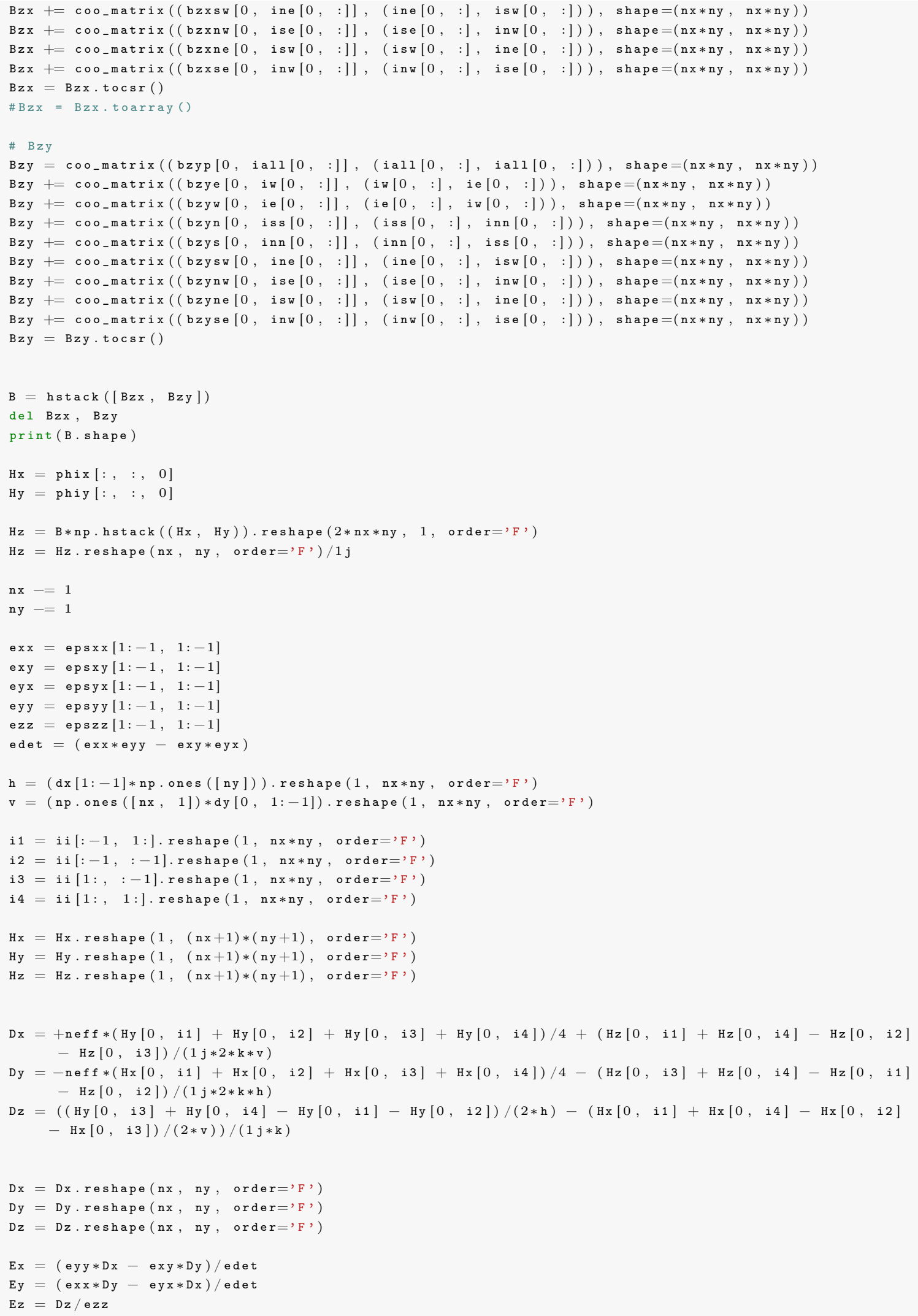




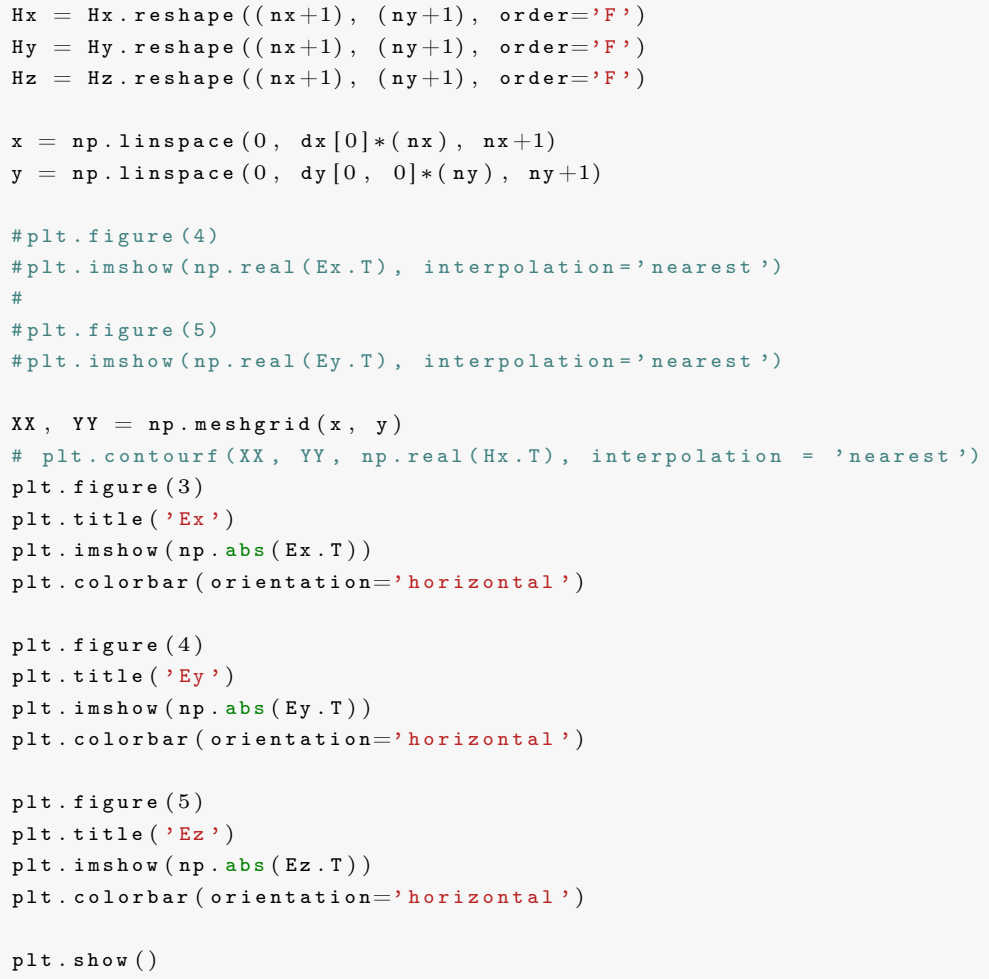

\section{Bibliography}

[1] Arman B Fallahkhair, Kai S Li, and Thomas E Murphy. Vector finite difference modesolver for anisotropic dielectric waveguides. Journal of Lightwave Technology, 26(11):1423-1431, 2008.

[2] https://www.photonics.umd.edu/software/wgmodes/. 


\section{Appendix E}

\section{Publications}

This is the list of the publications that this PhD thesis have generated.

[1] Zanzi, A., Brimont, A., Griol, A., Sanchis, P. and Marti, J., 2016. Compact and low-loss asymmetrical multimode interference splitter for power monitoring applications. Optics letters, 41(2), pp.227-229.

[2] Zanzi, A., Vagionas, C., Griol, A., Rosa, A., Lechago, S., Moralis-Pegios, M., Vyrsokinos, K., Pleros, N., Kraft, J., Sidorov, V. and Sirbu, B., 2019. Alignment tolerant, low voltage, $0.23 \mathrm{~V}$. cm, push-pull silicon photonic switches based on a vertical pn junction. Optics express, 27(22), pp.32409-32426.

[3] Zanzi, A., Sanchis, P., Marti, J. and Brimont, A., 2016. Design and Optimization of a High Speed Slow-Light Silicon Modulator in the O-Band. In Proc. 18th European Conf-Proc on Integrated Optics (ECIO).

[4] Zanzi, A., Rosa, A., Oriol, A., Sanchis, P., Marti, J. and Brimont, A., 2017, August. Advanced high speed slow-light silicon modulators in the O-band for low power optical interconnects in data centers. In 2017 IEEE 14th International Conference on Group IV Photonics (GFP) (pp. 149-150). IEEE.

[5] Zanzi, A., Brimont, A., Sanchis, P., and Marti, J., 2016, May. Design of a High Speed Silicon Modulator Based on a Vertical pn Junction at $1.31 \mu \mathrm{m}$ Wavelength. 2016 ECIO, 
[6] Brimont, A., Hu, X., Cueff, S., Romeo, P.R., Saint Girons, G., Griol, A., Zanzi, A., Sanchis, P. and Orobtchouk, R., 2015. Low-loss and compact silicon rib waveguide bends. IEEE Photonics Technology Letters, 28(3), pp.299-302.

[7] Moralis-Pegios, M., Terzenidis, N., Vagionas, C., Pitris, S., Chatzianagnostou, E., Brimont, A., Zanzi, A., Sanchis, P., Marti, J., Kraft, J. and Rochracher, K., 2017, February. A programmable Si-photonic node for SDN-enabled Bloom filter forwarding in disaggregated data centers. In Optical Interconnects XVII (Vol. 10109, p. 101090X). International Society for Optics and Photonics.

[8] Vyrsokinos, K., Moralis-Pegios, M., Vagionas, C., Brimont, A., Zanzi, A., Sanchis, P., Marti, J., Kraft, J., Rohracher, K., Dorrestein, S. and Bogdan, M., 2016, July. Single Mode Optical Interconnects for future data centers. In 2016 18th International Conference on Transparent Optical Networks (ICTON) (pp. 1-4). IEEE.

[9] K. Papatryfonos, D. R. Selviah, A. Maman, K. Hasharoni, A. Brimont, A. Zanzi, J. Kraft, V. Sidorov, M. Seifried, Y. Baumgartner, F. Horst, B. J. Offrein, K. Lawniczuk, R. G. Broeke, N. Terzenidis, G. Mourgias-Alexandris, M. Tang, A. J. Seeds, H. Liu, P. Sanchis, Member, IEEE, M. Moralis-Pegios, T. Manolis, K. Vyrsokinos, N. Pleros, B. Sirbu, Y. Eichhammer, H. Oppermann and T. Tekin. The L3MATRIX Project: Large Scale Silicon Photonics Matrix for low Power and Low Cost Data Centers (invited paper). Under review, Journal of Selected Topics in Quantum Electronics 


\section{List of Figures}

$1 \quad$ Big data traffic market forecast [1]. . . . . . . . . . . . . . . . 1

$2 \quad$ Forecast for silicon photonics market [5] (Silicon Photonics 2018 Report). . . . . 2

1.1 The graphs shows the variation of effective refractive index of a (a) strip waveguide and (b) rib waveguide at its width variation. For the strip waveguide the change in regime, from single mode to multimode is quite abrupt. On the other hand for the rib waveguide the transition is smoother. Therefore, the actual field distribution of the optical mode is required in order to better understand the real behavior of the waveguide, and its transition to the multi-mode regime. . . . . . . . . 10

1.2 Field distribution at $400 \mathrm{~nm}$ in a rib waveguide for the (a) fundamental and (b) first order mode. One can observe that the field distribution of the first order mode is mainly in the slab, which describe a situation in which the mode is not supported. Therefore the waveguide is single mode at $400 \mathrm{~nm}$ width. . . . . . . 11

1.3 a) Equivalent refractive index profile (n) of a rib waveguide bend versus radius (r) and b) schematic representation of the sources of losses in a rib bend waveguide. Namely, bending losses (Green arrow pointing towards the outer part of the bend, higher $\mathrm{r}$ values) and leakage losses produced by coupling to the slab mode (yellow arrows). Mode profiles for c) $20 \mu \mathrm{m}$ radii. A pronunced modal asymmetry can be observed. . . . . . . . . . . . . . . . . . . . . . . . . . . . . . . . . . 12

1.4 Shallow-etched bend loss vs radius; a local minimum can be observed when the radius is near $\sim 60 \mu \mathrm{m} . \quad \ldots \ldots \ldots$

1.5 The pictures summarises the mainly steps in the simulation of the phase shifter fabrication process. (a) Implantation of the dopants for the creation of the pn junction. (b) Definition of the waveguide. (c) Implantation of the doped regions.

(d) Oxide windows opening and final metallization. The pictures have been obtained from Silvaco (Appendix B) . . . . . . . . . . . . . . . . . . . . 14 
1.6 These figures exemplify (a) effective refractive index change, (b) propagation

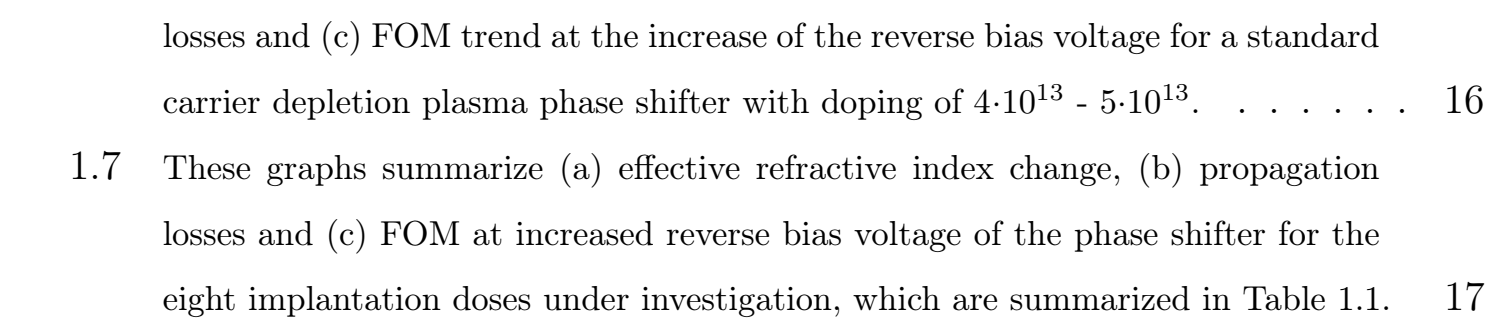

1.8 These graphs show (a) the dependance of the rise and fall time of the phase-shifter

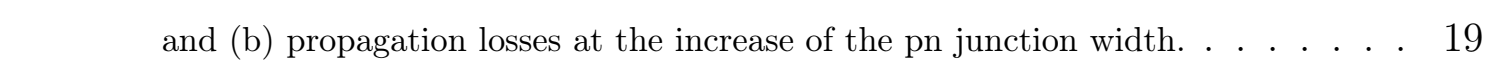

1.9 The graphs show the transient responses of the phase shifter in the two different

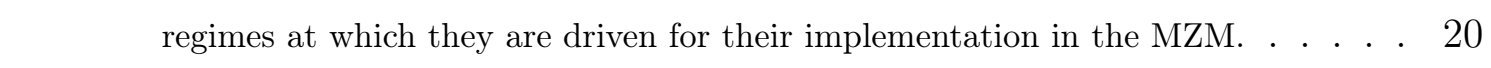

1.10 Process flow of the fabricated silicon modulator. (a) Implantation of the p-n junction doses, having as target the doses of $4 \cdot 10^{13}-5 \cdot 10^{13} \mathrm{~cm}^{-2}$. (b) Shallow implantation of the heavy doped regions $(\mathrm{p}++/ \mathrm{n}++)$ for the creation of ohmic \begin{tabular}{|c|}
\hline contact between the SOI region and tungsten vias. (c) Deposition of the oxide \\
\hline
\end{tabular} \begin{tabular}{|c|}
\hline and subsequent creation of the opening required for the vias. (d) Deposition of \\
\hline
\end{tabular}

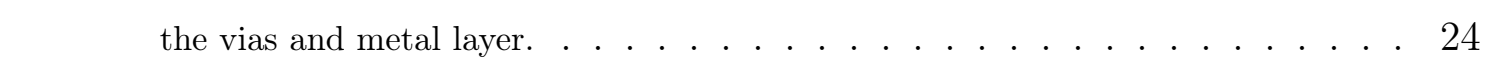

1.11 (a) 3D layout of phaseshifter. (b) SEM image of the cross-section of the fabricate modulator. ........................... . 25

1.12 Schematic of the DC and RF setup used for the characterization of the device. . 26

1.13 (a) Fabricated device (b) DC response of the MZM from $+0.8 \mathrm{~V}$ to $-3 \mathrm{~V}$. . . . . 26

$1.14 \mathrm{RF}$ response of the MZM at different biases (a) $1.6 \mathrm{~V}$ (b) $2.3 \mathrm{~V}$ (c) $3.2 \mathrm{~V}$ and different speeds. It is to notice that increasing the speed of the modulator the ER decreases (for constant voltage) and increasing the voltage biases, at a constant speed, the ER increases. . . . . . . . . . . . . . . . . . . . . . . . . . . . 27

1.15 (a), (b), (c) S-parameters and (d), (e), (f) impedance of the transmission line for

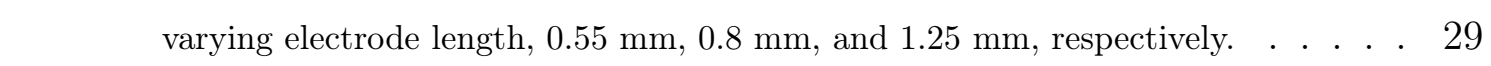

1.16 Microwave loss for varying single electrode test structure length and type. DD refers to the dual drive electrodes used in the designed modulator. . . . . . . . 29

1.17 Metal layer stack configuration. . . . . . . . . . . . . . . . . 29

1.18 S-parameter simulations of (a) the actual fabricated layer-stack-up of the MZM and (b) optimized electrodes for future fabrication runs. . . . . . . . . . . . 30

2.1 (a) Vertical cross-section of the waveguide. (b) Schematic and geometry of a standard MMI. . . . . . . . . . . . . . . . . 36 
2.2 (a) 2D schematic of a 1-by-2 A-MMI power splitter, which shows the design \begin{tabular}{|c|}
\hline parameters. (b) Distribution of the magnetic field $\left(H_{y}\right)$ in the multimode region \\
\hline
\end{tabular} of the asymmetrical MMI $(15 / 85 \mathrm{~A}-\mathrm{MMI})\left(\Delta^{*}=0.2 \mu \mathrm{m}\right) . \quad$. . . . . . . . . . . . . 38

2.3 (a) SEM image of the A-MMI splitter with a ratio of $30 / 70\left(\theta \sim 83^{\circ}\right)$. (b) Snapshot

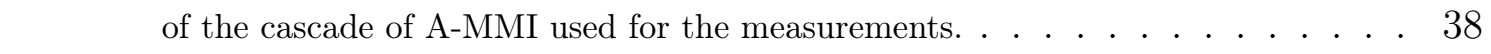

2.4 (a) Photo of the DUT and (b) schematic of the setup used for the characterization. 39

2.5 (a) Wavelength dependence of the average splitting ratio (R) in the range 1540-

\begin{tabular}{l}
\hline $1580 \mathrm{~nm}$, where we can observe the MMI broadband operation. (b) Wavelength \\
\hline dependence of the device losses in the range $1540-1580 \mathrm{~nm}$. The losses are in \\
\hline the range $\sim 0.4-0.8 \mathrm{~dB}$ for the four considered geometries. (c) Graph showing \\
\hline the details of the dependence of the splitting ratio upon $\theta$ at $1550 \mathrm{~nm}$. The \\
\hline experimental values deviate exhibit a deviation up to a $\sim 3 \%$ from the 3D-FDTD \\
\hline simulations results. . . . . . . . . . . . . . . . . . . . . 40
\end{tabular}

$2.6 \quad$ (a) 2D geometry of the vertical pn junction in rib configuration. (b) Schematic \begin{tabular}{|c|}
\hline of the MZI with single phase shifter and arbitrary splitting ratio. (c) $2 \mathrm{D}$ cross \\
\hline
\end{tabular} section of the simulated pn junction (Silvaco). (d) Net doping profile of the phase shifter. . . . . . . . . . . . . . . . . . . . . . . . . . 41

$2.7 \quad$ (a) Splitting ratio dependence of insertion losses and extinction ratio. (b) Relation

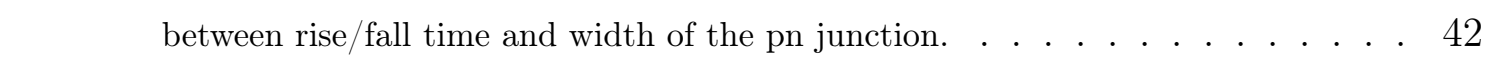

2.8 Graph shows a detail of the dependence of the splitting ratio from $\theta$ at $1.31 \mu \mathrm{m}$. . 43

2.9 (a) Layout overview of the device. (b) Schematic of the characterisation setup for

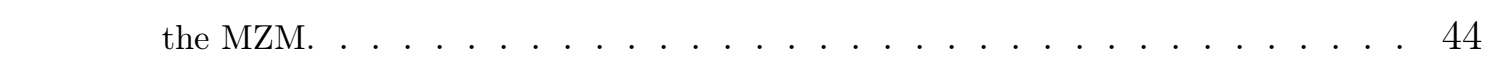

2.10 (a) Graph shows the propagation losses of the doped phase shifter $(\sim 6-7 \mathrm{~dB} / \mathrm{mm})$ \begin{tabular}{|c|}
\hline compared to that the of a reference standard rib waveguide $(\sim 1 \mathrm{~dB} / \mathrm{mm})$ of the \\
\hline same chip. It shows the high contribution of the doping on the overall power \\
\hline budget of the modulator. (b) Propagation losses caused by the doping inside the \\
\hline phase shifter only are reported. The measured values show good agreement with \\
\hline
\end{tabular}

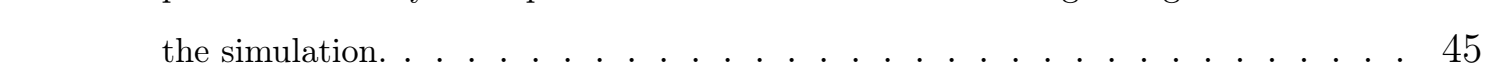

2.11 (a) DC response of a 65/35 MZM. (b) Eye-diagram of the MZM @ 4 Gbit/s (ER \begin{tabular}{|l|}
\hline$=6.25 \mathrm{~dB})$. (c) Eye-diagram of the MZM @ 5 Gbit $/ \mathrm{s}(\mathrm{ER}=4.40 \mathrm{~dB})$ operating \\
\hline at $1305 \mathrm{~nm}$. . . . . . . . . . . . . . . . . . . . . . . . . . . . . . . 46
\end{tabular} 
2.12 Comparison of the analytical and measured results of the modulators, in terms of insertion losses and extinction ratio versus reference waveguide (WG) - phase shifter (PS) power ratio . . . . . . . . . . . . . . . . . . . . . 47

3.1 (a) Vertical cross-section of the waveguide. (b) Schematic and geometry of the $1 \mathrm{D} \mathrm{PhC} \ldots \ldots \ldots \ldots \ldots \ldots \ldots 5$

3.2 (a) Cross-sections of the optical mode for the (a) $400 \mathrm{~nm}$ and (b) $1 \mu \mathrm{m}$ section of the PhC. Effective refractive index are respectively $n_{\text {eff }}^{W i}=2.8248$ and $n_{\text {eff }}^{W e}=2.9398 .57$

$3.3 \quad$ Band structure of the designed 1D-PhC and related optical modes. . . . . . . . 57

3.4 Sketch of the 1D tapers under investigation: (a) Standard 1D PhC; (b) Linear taper; (c) Blackman taper and (d) Raised Sine taper. . . . . . . . . . . . . 58

$3.5 \quad$ 2D FDTD of the designed structures $(\mathrm{N}=200)$ with vEIM implementation both in (a) linear and (b) logarithmic scales. . . . . . . . . . . . . . . . 59

3.6 (a), (b), (c) SEM images of the fabricated PhC at NTC; (d) Comparison between \begin{tabular}{|c|}
\hline original design, fabricated device and simulations, with new dimensions. The \\
\hline
\end{tabular} good agreement verifies the change induced by the fabrication process on PhC. The modified dimensions are: $L_{i} \simeq 90 \mathrm{~nm}, W_{i} \simeq 450 \mathrm{~nm}$ and $\Lambda \simeq 231 \mathrm{~nm}$. . . . . . 60

$3.7 \quad$ Picture and schematic of the setup used for the characterization of the slow-light - structures. . . . . . . . . . . . . . . . . . 6 61

3.8 (a) Example MZI spectrum with corresponding calculated values of group index, up to $n_{g} \simeq 9$-10 near the slow-light regions, which are highlighted by the red areas.

(b) Example of transmission spectra and corresponding values of propagation \begin{tabular}{|c|}
\hline losses. Similarly to $n_{g}$, the losses increases significantly when approaching the \\
\hline
\end{tabular} slow-light regions. . . . . . . . . . . . . . . . . . 62

3.9 Normalized transmission spectra of the measured PhC: (a) No apodization, (b) Blackman, (c) Raised Sine and (d) Linear. Four different length were measured $(1000,2000,3000$ and 4000 periods) with a $\Lambda \simeq 230 \mathrm{~nm}$. . . . . . . . . . 63

3.10 Propagation losses of the measured spectra: (a) No apodization, (b) Blackman, \begin{tabular}{|c|}
\hline (c) Raised Sine and (d) Linear. Four different length were measured (1000, 2000, \\
\hline
\end{tabular} 3000 and 4000 periods) with $\mathrm{a} \Lambda \simeq 231 \mathrm{~nm}$. . . . . . . . . . 65

3.11 Group index $\left(n_{g}\right)$ of the measured spectra: (a) No apodization, (b) Blackman, \begin{tabular}{|c|}
\hline (c) Raised Sine and (d) Linear. Four different length were measured (1000, 2000, \\
\hline
\end{tabular} 3000 and 4000 periods) with a $\Lambda \simeq 231 \mathrm{~nm} . \ldots \ldots \ldots 67$ 
3.12 The losses and $n_{g}$ values has been related and using a linear interpolation the FOM

\begin{tabular}{|l|}
\hline for each one of the bands as been calculated.(a) No apodization, (b) Blackman, \\
\hline (c) Raised Sine and (d) Linear. . . . . . . . . . . . . . . . . . . . . . . . . . . . 68
\end{tabular}

3.13 FOM values for the progressive Blackman layout: (a) $1^{\text {st }}$ band, (b) $2^{\text {nd }}$ band and

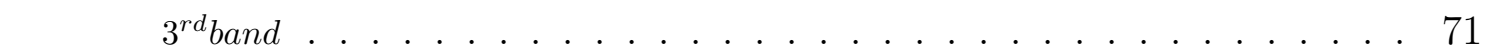

3.14 FOM values for the progressive Raised Sine layout: (a) $1^{\text {st }}$ band, (b) $2^{\text {nd }}$ band

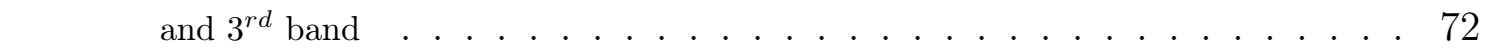

3.15 FOM values for the progressive Raised Sine layout: (a) $1^{\text {st }}$ band, (b) $2^{\text {nd }}$ band

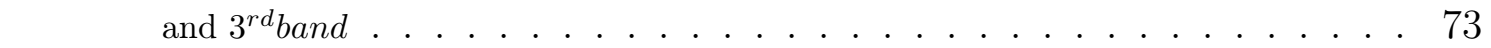

3.16 (a) The graph shows the $\Delta \mathrm{n}_{e f f}$ distribution induced by the use of a single apodization functions on a 200 period $\mathrm{PhC}$. The average value is well above the reference value of the $400 \mathrm{~nm}$ slab waveguide case. (b) The image depicts a layout example $(\mathrm{N}=200)$ with double tapering functions. . . . . . . . . . . . . . . . . . . . 76

3.17 (a) Simulated transmission spectra for $W_{i} \leq 400 \mathrm{~nm}$ and (b) relative space-averaged distribution of $\Delta n_{e f f}$ for $\mathrm{N}=200$. (c) Simulated transmission spectra for $W_{i} \geq 400$

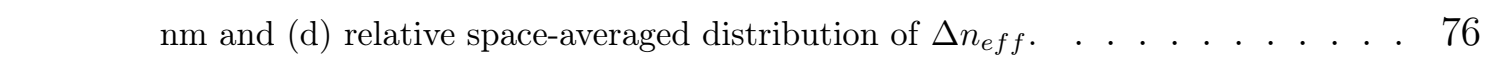

3.18 Band structure and relative optical modes for $W_{i}=250 \mathrm{~nm}$ (Left) and $W_{i}=300$ $\mathrm{nm}$ (Right) . . . . . . . . . . . . . . . . . . . . . . . . . 77

3.19 Normalized transmission spectra of the measured $\mathrm{PhC}$ with double apodisation: (a) Blackman, (b) Raised Sine and (c) Linear. Three different geometries have been measured with $W_{i}=250,300$ and $350 \mathrm{~nm}$. . . . . . . . . . . . . . . . . 78

3.20 Propagation losses of the measured $\mathrm{PhC}$ with double apodisation: (a) Blackman, (b) Raised Sine and (c) Linear. Three different geometries have been measured with $W_{i}=250,300$ and $350 \mathrm{~nm} .$. . . . . . . . . . . . . . . . . . . . . . . . 80

3.21 Group index distribution of the measured $\mathrm{PhC}$ with double apodisation: (a)

Blackman, (b) Raised Sine and (c) Linear. Three different geometries have been measured with $W_{i}=250,300$ and $350 \mathrm{~nm} . \quad$. . . . . . . . . . . . . . . . . . 81

3.22 FOM values for both bands of the Blackman double apodization geometry: (a) $W_{i}=250 \mathrm{~nm},(\mathrm{~b}) W_{i}=300 \mathrm{~nm}$ and $(\mathrm{c})=350 \mathrm{~nm}$. . . . . . . . . . . . . . . 82

3.23 FOM values for both bands of the Raised Sine double apodization geometry: (a) $W_{i}=250 \mathrm{~nm},(\mathrm{~b}) W_{i}=300 \mathrm{~nm}$ and $(\mathrm{c})=350 \mathrm{~nm}$. . . . . . . . . . . . . . . 83

3.24 FOM values for both bands of the Linear double apodization geometry: (a) $W_{i}$ \begin{tabular}{|l}
\hline \\
\hline
\end{tabular} $250 \mathrm{~nm},(\mathrm{~b}) W_{i}=300 \mathrm{~nm}$ and $(\mathrm{c})=350 \mathrm{~nm}$. . . . . . . . . . . . . . . . . . 84 
A.1 Transmission spectra of the measured 1D-PhC with partial tapers: (a) Blackman, (b) Raised Sine and (c) Linear. Two different length were measured: 750 and 1500. The full tapered $\mathrm{PhC}(2000)$ has been added for comparison. . . . . . . . . . . 97

A.2 Propagation losses of the measured 1D-PhC with partial tapers: (a) Blackman, (b) Raised Sine and (c) Linear. Two different length were measured: 750 and 1500. The full tapered $\mathrm{PhC}(2000)$ has been added for comparison. . . . . . . . 98

A.3 Group index $\left(n_{g}\right)$ of the measured 1D-PhC with partial tapers: (a) Blackman, (b) Raised Sine and (c) Linear. Two different length were measured: 750 and 1500. The full tapered $\mathrm{PhC}(2000)$ has been added for comparison. . . . . . . . 99 


\section{List of Tables}

1.1 This table displays the doses that have been taken into account for the design of the phase shifter. . . . . . . . . . . . . . . . . . . . . . . . 16

1.2 This table summarizes the doses that have been taken into account for the design of the phase shifter. . . . . . . . . . . . . . . . . . . . . . . . . . . . . . 19

1.3 This table summarizes the specifications in DC for the two doses under investigation. 22

1.4 This table summarizes the specifications at high frequency for the two doses under investigation . . . . . . . . . . . . . . . . . . . . . 22

1.5 This table shows how an increase in desired ER affects the footprint and insertion losses of the phase-shifter (in DC). . . . . . . . . . . . . . . . . . . . . . . 23

1.6 $\quad$ This table shows how an increase in desired ER affects the footprint and insertion losses of the phase-shifter (in RF). . . . . . . . . . . . . . . . . . . . . . 23

$3.1 \quad$ Values of FOM for each design and each band . . . . . . . . . . . . 68

3.2 Values of effective refractive index $\left(n_{e f f}\right)$ for each one of the optical mode of the $\operatorname{PhC}\left(\Lambda=230 \mathrm{~nm}, L_{i}=120 \mathrm{~nm}\right.$ and $\left.L_{e}=110 \mathrm{~nm}\right)$. The values are calculated using the PWE method. . . . . . . . . . . . . . 69

$3.3 \quad$ FOM values for the progressive Blackman layout. Full tapered and untapered versions are present for completeness. . . . . . . . . . . . . . . . . . . . . . 70

$3.4 \quad$ FOM values for the progressive Raised Sine layout. Full tapered and untapered versions are present for completeness. . . . . . . . . . . . . . . . . . . . . . 72

$3.5 \quad$ FOM values for the progressive Linear layout. Full tapered and untapered versions are present for completeness. . . . . . . . . . . . . . . . . . . . . . 74

$3.6 \quad$ FOM values for the Blackman double apodisation geometries $\left(W_{i}=250,300,350\right.$ $\mathrm{nm}$ ) for the $1^{\text {st }}$ and $2^{\text {nd }}$ band. . . . . . . . . . . . . . . . . . . . . . . . . . 82 
3.7 FOM values for the Raised Sine double apodisation geometries $\left(W_{i}=250,300\right.$, $350 \mathrm{~nm}$ ) for the $1^{\text {st }}$ and $2^{\text {nd }}$ band. . . . . . . . . . . . . . . . . . 83

3.8 FOM values for the Linear double apodisation geometries $\left(W_{i}=250,300,350\right.$ $\mathrm{nm}$ ) for the $1^{\text {st }}$ and $2^{\text {nd }}$ band. . . . . . . . . . . . . . . . . . . . . 84 